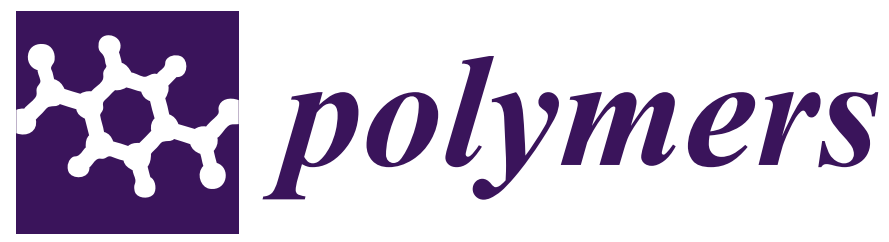

\title{
Young Talents in Polymer Science
}

Edited by Alexander Böker and Frank Wiesbrock Printed Edition of the Special Issue Published in Polymers 


\section{Young Talents in \\ Polymer Science}

\section{Special Issue Editors}

Alexander Böker

Frank Wiesbrock

MDPI • Basel • Beijing • Wuhan • Barcelona • Belgrade 
Special Issue Editors

$\begin{array}{ll}\text { Alexander Böker } & \text { Frank Wiesbrock } \\ \text { Universität Potsdam } & \text { Polymer Competence Center Leoben GmbH } \\ \text { Germany } & \text { Austria }\end{array}$

Editorial Office

MDPI AG

St. Alban-Anlage 66

Basel, Switzerland

This edition is a reprint of the Special Issue published online in the open access journal Polymers (ISSN 2073-4360) from 2016-2017 (available at: http://www.mdpi.com/journal/polymers/special_issues/young_talents).

For citation purposes, cite each article independently as indicated on the article page online and as indicated below:

Author 1; Author 2. Article title. Journal Name Year, Article number, page range.

First Edition 2017

ISBN 978-3-03842-458-1 (Pbk)

ISBN 978-3-03842-459-8 (PDF)

Articles in this volume are Open Access and distributed under the Creative Commons Attribution license (CC BY), which allows users to download, copy and build upon published articles even for commercial purposes, as long as the author and publisher are properly credited, which ensures maximum dissemination and a wider impact of our publications. The book taken as a whole is (C) 2017 MDPI, Basel, Switzerland, distributed under the terms and conditions of the Creative Commons license CC BY-NC-ND (http://creativecommons.org/licenses/by-nc-nd/4.0/). 


\section{Table of Contents}

About the Special Issue Editors ...................................................................................................

Preface to "Young Talents in Polymer Science" ....................................................................................vii

Spencer D. Brucks, Jessica L. Freyer, Tristan H. Lambert and Luis M. Campos

Influence of Substituent Chain Branching on the Transfection Efficacy of Cyclopropenium-Based

Polymers

Reprinted from: Polymers 2017, 9(3), 79; doi: doi:10.3390/polym9030079 1

Lihua Guo, Xinyu Jing, Shuoyan Xiong, Wenjing Liu, Yanlan Liu, Zhe Liu and Changle Chen Influences of Alkyl and Aryl Substituents on Iminopyridine Fe(II)- and Co(II)-Catalyzed Isoprene Polymerization

Reprinted from: Polymers 2016, 8(11), 389; doi: 10.3390/polym8110389 . .10

Kanykei Ryskulova, Anupama Rao Gulur Srinivas, Thomas Kerr-Phillips, Hui Peng, David Barker, Jadranka Travas-Sejdic and Richard Hoogenboom

Multiresponsive Behavior of Functional Poly( $p$-phenylene vinylene)s in Water Reprinted from: Polymers 2016, 8(10), 365; doi: 10.3390/polym8100365.

\section{Anna P. Constantinou, Hanyi Zhao, Catriona M. McGilvery, Alexandra E. Porter and} Theoni K. Georgiou

A Comprehensive Systematic Study on Thermoresponsive Gels: Beyond the Common Architectures of Linear Terpolymers

Reprinted from: Polymers 2017, 9(1), 31; doi: 10.3390/polym9010031

Kyriakos Christodoulou, Epameinondas Leontidis, Mariliz Achilleos, Christiana Polydorou and Theodora Krasia-Christoforou

Semi-Interpenetrating Polymer Networks with Predefined Architecture for Metal Ion

Fluorescence Monitoring

Reprinted from: Polymers 2016, 8(12), 411; doi: 10.3390/polym8120411

Siew Yin Chan, Wee Sim Choo, David James Young and Xian Jun Loh

Thixotropic Supramolecular Pectin-Poly(Ethylene Glycol) Methacrylate (PEGMA) Hydrogels

Reprinted from: Polymers 2016, 8(11), 404; doi: 10.3390/polym8110404.

Tianfeng Shen, Piming Ma, Qingqing Yu, Weifu Dong and Mingqing Chen

The Effect of Thermal History on the Fast Crystallization of Poly(L-Lactide) with Soluble-Type

Nucleators and Shear Flow

Reprinted from: Polymers 2016, 8(11), 431; doi: 10.3390/polym8120431

Sai Aditya Pradeep, Hrishikesh Kharbas, Lih-Sheng Turng, Abraham Avalos, Joseph G. Lawrence and Srikanth Pilla

Investigation of Thermal and Thermomechanical Properties of Biodegradable PLA/PBSA

Composites Processed via Supercritical Fluid-Assisted Foam Injection Molding

Reprinted from: Polymers 2017, 9(1), 22; doi: 10.3390/polym9010022.. 
Jenny Alongi and Federico Carosio

All-Inorganic Intumescent Nanocoating Containing Montmorillonite Nanoplatelets in Ammonium Polyphosphate Matrix Capable of Preventing Cotton Ignition

Reprinted from: Polymers 2016, 8(12), 430; doi: 10.3390/polym8120430. 107

Benjamin Weber, Christine Seidl, David Schwiertz, Martin Scherer, Stefan Bleher, Regine Süss and Matthias Barz

Polysarcosine-Based Lipids: From Lipopolypeptoid Micelles to Stealth-Like Lipids in Langmuir Blodgett Monolayers

Reprinted from: Polymers 2016, 8(12), 427; doi: 10.3390/polym8120427. .121

\section{Takahiro Sakaue}

Dynamics of Polymer Translocation: A Short Review with an Introduction of Weakly-Driven Regime Reprinted from: Polymers 2016, 8(12), 424; doi: 10.3390/polym8120424.

Hannah C. Bygd and Kaitlin M. Bratlie

Investigating the Synergistic Effects of Combined Modified Alginates on Macrophage Phenotype Reprinted from: Polymers 2016, 8(12), 422; doi: 10.3390/polym8120422. 


\section{About the Special Issue Editors}

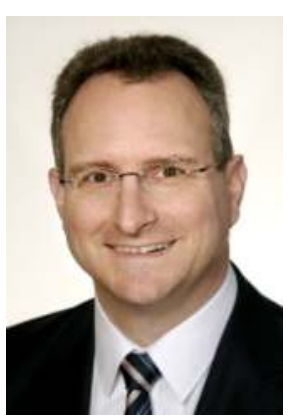

Alexander Böker is Director of the Fraunhofer-Institute for Applied Polymer Research (IAP) and holds the Chair for Polymer Materials and Polymer Technology at the University of Potsdam, Germany. He studied chemistry at the Johannes Gutenberg University, Mainz, and Cornell University, Ithaca, NY. He received his $\mathrm{PhD}$ from Bayreuth University in 2002 working with Prof. G. Krausch and Prof. Axel H.E. Müller. From 2002-2004, he was a postdoctoral fellow with Thomas P. Russell at the University of Massachusetts, Amherst. In 2006, he received a Lichtenberg-Professorship funded by the VolkswagenStiftung and in 2015 the ERC Consolidator Grant. Alexander Böker has published more than 130 papers in peer-reviewed journals. The main research interests of his group include guided self-assembly of block copolymer systems, hierarchical (bio)nanoparticle assemblies and the control of self-assembly processes via external fields.

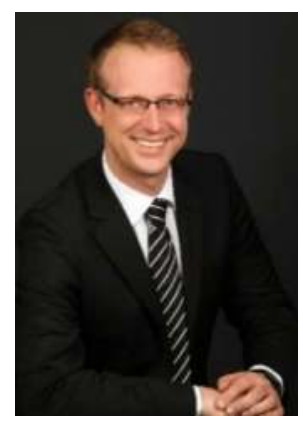

Frank Wiesbrock received his doctorate at the Technical University of Munich, Germany, in 2003 for his work on metal B-amino carboxylates under the supervision of $\mathrm{H}$. Schmidbaur. His subsequent biannual postdoctoral stay with U.S. Schubert at the Eindhoven Technical University focused on block copoly(2-oxazoline)s from microwave-assisted synthesis. He worked as a project manager at Chemspeed Technologies AG in Augst, Switzerland, and returned to academia in 2007 as a Marie Curie ToK researcher with T. Calogeropoulou at the National Hellenic Research Foundation in Athens, Greece. Since 2008, he has been an assistant professor/lecturer at the Graz University of Technology, Austria, where he completed his Habilitation in 2012. Currently he is employed as a senior researcher at the Polymer Competence Center Leoben (PCCL), Austria. His research interests comprise microwave-assisted polymerizations, poly(2-oxazoline)s, biopolymers and biocompatible polymers, and nanocomposites for electronic applications. 



\section{Preface to "Young Talents in Polymer Science"}

Inspired by the excellent nominations for the 1st Young Investigator Award, the journal Polymers compiled a selection of publications of these successful young polymer scientists in one Special Issue. It was an easy choice for us to publish the recent research successes of the nominated high-ranking scientists as an additional hardcover book to share with you, our dear readers. The Special Issue as well as the book contains 12 contributions in the form of 11 original research articles and one review, highlighting the exciting plethora of current research topics in the area of polymer science.

Polymer Synthesis and Macromolecular Architectures. In the article 'Influence of Substituent Chain Branching on the Transfection Efficacy of Cyclopropenium-Based Polymers' by the 2016 award winner, Luis M. Campos [1] and colleagues, the authors correlate the degree of alkyl chain branching of cationic polymers based on trisaminocyclopropenium ions with their cytotoxicity, transfection efficacy, and their performance as nonviral vectors in gene therapy [2]. Changle Chen et al. describe a series of iminopyridine FeII and CoII complexes as catalysts for the polymerization of isoprene in the publication 'Influences of Alkyl and Aryl Substituents on Iminopyridine Fe(II)- and Co(II)-Catalyzed Isoprene Polymerization' with particular focus on the cis-1,4-selectivity of the corresponding poly(isoprene)s [3]. Richard Hoogenboom and colleagues report the multiresponsive behavior of different 2,5-substituted poly(phenylene vinylene)s (with varying amounts of carboxylic acid units) upon stimuli such as $\mathrm{pH}$ changes, temperatures, and the addition of divalent ions in the article 'Multiresponsive Behavior of Functional Poly(p-phenylene vinylene)s in Water' [4].

Methacrylate-Based Crosslinked Polymers and (Hydro-)Gels. Theoni K. Georgiou et al. describe the synthesis of different methacrylate-based diblock terpolymers and correlate the copolymers' architectures with their thermoresponsive properties and sol-gel transitions in the publication 'A Comprehensive Systematic Study on Thermoresponsive Gels: Beyond the Common Architectures of Linear Terpolymers' [5]. In the article 'Semi-Interpenetrating Polymer Networks with Predefined Architecture for Metal Ion Fluorescence Monitoring', Theodora Krasia-Christoforou and coworkers report the preparation of methacrylate-based semi-interpenetrating networks and their applicability as sorbents and fluorescence chemosensors [6]. David James Young, Xian Jun Loh and colleagues describe the grafting of methoxy-poly(ethylene glycol) methacrylate onto pectin and the subsequent formation of the corresponding supramolecular hydrogels with thixotropic properties in the study 'Thixotropic Supramolecular Pectin-Poly(Ethylene Glycol) Methacrylate (PEGMA) Hydrogels' [7].

Polymer Blends and Composites. Piming Ma et al. report the promotion of the crystallization process of a blend composed of poly(L-lactide) and a phenyloxalamide-based nucleator in the publication 'The Effect of Thermal History on the Fast Crystallization of Poly(L-Lactide) with Soluble-Type Nucleators and Shear Flow' [8]. Srikanth Pilla and colleagues summarize the processing of blends of poly(lactic acid) and poly(butylene succinate-co-adipate) for the production of biobased polymer foams in the report 'Investigation of Thermal and Thermomechanical Properties of Biodegradable PLA/PBSA Composites Processed via Supercritical Fluid-Assisted Foam Injection Molding' [9]. A completely inorganic intumescent flame retardant nanocomposite is reported by Jenny Alongi et al. in the study 'AllInorganic Intumescent Nanocoating Containing Montmorillonite Nanoplatelets in Ammonium Polyphosphate Matrix Capable of Preventing Cotton Ignition' [10].

Medic(in)al Applications of Polymers. Matthias Barz and coworkers describe the synthesis of polysarcosine-based lipids by nucleophilic ring-opening polymerizations and evaluate their potential to substitute poly(ethylene glycol)-based lipopolymers in the publication 'Polysarcosine-Based Lipids: From Lipopolypeptoid Micelles to Stealth-Like Lipids in Langmuir Blodgett Monolayers' [11]. A concise summary of the current state-of-the-art knowledge of polymer translocation and a crossover scenario connecting unbiased and strongly-driven regimes is provided by Takahiro Sakaue in the review 'A Short Review with an Introduction of Weakly-Driven Regime' [12]. The effect of various chemically modified alginates on the reprogramming capabilities of macrophages is reported in the publication 'Investigating the Synergistic Effects of Combined Modified Alginates on Macrophage Phenotype' by Kaitlin M. Bratlie et al. [13]. 
Enjoy reading this collection of scientifically high-end research highlights, addressing the current 'hot' research topics!

\section{Alexander Böker and Frank Wiesbrock \\ Special Issue Editors}

\section{References}

1. Böker, A. Announcement of the 2016 Polymers Young Investigator Award. Polymers 2016, 8, 2, doi:10.3390/polym8030065.

2. Brucks, S.D.; Freyer, J.L.; Lambert, T.H.; Campos, L.M. Influence of Substituent Chain Branching on the Transfection Efficacy of Cyclopropenium-Based Polymers. Polymers 2017, 9, 9, doi:10.3390/polym9030079.

3. Guo, L.; Jing, X.; Xiong, S.; Liu, W.; Liu, Y.; Liu, Z.; Chen, C. Influences of Alkyl and Aryl Substituents on Iminopyridine Fe(II)- and Co(II)-Catalyzed Isoprene Polymerization. Polymers 2016, 8, 12, doi:10.3390/polym8110389.

4. Ryskulova, K.; Rao Gulur Srinivas, A.; Kerr-Phillips, T.; Peng, H.; Barker, D.; Travas-Sejdic, J.; Hoogenboom, R. Multiresponsive Behavior of Functional Poly(p-phenylene vinylene)s in Water. Polymers 2016, 8, 13, doi:10.3390/polym8100365.

5. Constantinou, A.P.; Zhao, H.; McGilvery, C.M.; Porter, A.E.; Georgiou, T.K. A Comprehensive Systematic Study on Thermoresponsive Gels: Beyond the Common Architectures of Linear Terpolymers. Polymers 2017, 9, 17, doi:10.3390/polym9010031.

6. Christodoulou, K.; Leontidis, E.; Achilleos, M.; Polydorou, C.; Krasia-Christoforou, T. SemiInterpenetrating Polymer Networks with Predefined Architecture for Metal Ion Fluorescence Monitoring. Polymers 2016, 8, 13, doi:10.3390/polym8120411.

7. Chan, S.Y.; Choo, W.S.; Young, D.J.; Loh, X.J. Thixotropic Supramolecular Pectin-Poly(Ethylene Glycol) Methacrylate (PEGMA) Hydrogels. Polymers 2016, 8, 12, doi:10.3390/polym8110404

8. Shen, T.; Ma, P.; Yu, Q.; Dong, W.; Chen, M. The Effect of Thermal History on the Fast Crystallization of Poly(L-Lactide) with Soluble-Type Nucleators and Shear Flow. Polymers 2016, 8, 12, doi:10.3390/polym8120431.

9. Pradeep, S.A.; Kharbas, H.; Turng, L.-S.; Avalos, A.; Lawrence, J.G.; Pilla, S. Investigation of Thermal and Thermomechanical Properties of Biodegradable PLA/PBSA Composites Processed via Supercritical Fluid-Assisted Foam Injection Molding. Polymers 2017, 9, 18, doi:10.3390/polym9010022.

10. Alongi, J.; Carosio, F. All-Inorganic Intumescent Nanocoating Containing Montmorillonite Nanoplatelets in Ammonium Polyphosphate Matrix Capable of Preventing Cotton Ignition. Polymers 2016, 8, 14, doi:10.3390/polym8120430.

11. Weber, B.; Seidl, C.; Schwiertz, D.; Scherer, M.; Bleher, S.; Süss, R.; Barz, M. Polysarcosine-Based Lipids: From Lipopolypeptoid Micelles to Stealth-Like Lipids in Langmuir Blodgett Monolayers. Polymers 2016, 8, 14, doi:10.3390/polym8120427.

12. Sakaue, T. Dynamics of Polymer Translocation: A Short Review with an Introduction of WeaklyDriven Regime. Polymers 2016, 8, 12, doi:10.3390/polym8120424.

13. Bygd, H.C.; Bratlie, K.M. Investigating the Synergistic Effects of Combined Modified Alginates on Macrophage Phenotype. Polymers 2016, 8, 16, doi:10.3390/polym8120422. 




\title{
Communication
}

\section{Influence of Substituent Chain Branching on the Transfection Efficacy of Cyclopropenium-Based Polymers}

\author{
Spencer D. Brucks, Jessica L. Freyer, Tristan H. Lambert and Luis M. Campos* \\ Department of Chemistry, Columbia University, 3000 Broadway, New York, NY 10027, USA; \\ sdb2147@columbia.edu (S.D.B.); jlf2176@columbia.edu (J.L.F.); tl2240@columbia.edu (T.H.L.) \\ * Correspondence: lcampos@columbia.edu; Tel.: +1-212-854-9561
}

Academic Editor: Wei Min Huang

Received: 6 January 2017; Accepted: 21 February 2017; Published: 24 February 2017

\begin{abstract}
The realization of gene therapy relies on the development of delivery vectors with high efficiency and biocompatibility. With a multitude of structures accessible, the core challenge is precisely tuning vector structure to probe and optimize structure-property relationships. Employing a modular strategy, two pairs of cationic polymers based on the trisaminocyclopropenium (TAC) ion were synthesized where the substituents differ in the degree of alkyl chain branching. All TAC-based polymers exhibited higher transfection efficiencies than the untreated controls, with variable in vitro toxicities. Considering both cytotoxicity and transfection efficacy, an optimal nonviral vector was identified. Our studies highlight the importance of exercising precise control over polymer structure, both in terms of backbone identity and substituent nature, and the necessity of a robust, modular platform from which to study them.
\end{abstract}

Keywords: gene delivery; polyelectrolyte; nonviral vectors; structure-property relationships

\section{Introduction}

The fundamental challenge of gene therapy is the design of efficient, biocompatible delivery vectors [1-3]. Since the concept of gene delivery was first introduced, both viral and nonviral options have been explored, with each presenting their own benefits and drawbacks $[1,4]$. While viral technologies demonstrate high gene expression, their clinical translation has been limited by immunogenicity, lack of selectivity, and synthetic difficulty [2,5]. On the contrary, nonviral vectors elicit a reduced immunogenic response, but thus far have shown lower delivery efficiencies. The development of an optimized nonviral vector, balancing safety with efficiency, thus remains a critical goal to the realization of gene therapy $[1,2,6]$.

Cationic polymers are one of the most commonly studied nonviral vectors due to their high stability and capacity to tune macromolecular composition and architecture $[7,8]$. Linear polyethylenimine (PEI) was the first cationic polymer observed to rapidly complex negatively charged nucleic acids into polyplexes [9]. In the subsequent decades, there has been a great proliferation of cationic structures shown to bind and transfect nucleic acids. In addition to increasing the library of cationic polymers available, these research efforts have also uncovered some design principles towards optimizing polymeric structure for transfection [1,7]. In general, lower molecular weight polymers demonstrate lowered cytotoxicity than their high molecular weight counterparts [10-13], and increasing the degree of polymer chain branching appears to heighten both transfection efficacy and toxicity [14-17]. Wang and coworkers have recently highlighted the great potential of branched and dendritic polymer topologies to enhance transfection efficiency [18-21]. With the advent of controlled polymerization 
techniques, the challenge has now become fine-tuning polymer structure, size, and architecture to probe detailed structure-property relationships among the various polymers accessible.

We recently reported a modular platform to functionalize polymers of various sizes and architectures with a host of bis(dialkylamino)cyclopropenium chloride (BACCl) derivatives [22]. In an efficient post-polymerization click reaction, polymers bearing pendent or main-chain secondary amines were quantitatively transformed into the aromatic trisaminocyclopropenium (TAC) ion. We found that some of the resulting TAC polymers were biocompatible and efficient transfection agents and, furthermore, the ability to precisely tune polymer structure had significant effects on macromolecular properties, including transfection efficiency.

Herein, we extend these results by probing the effect of the substituted alkylamino chain's degree of branching on cytotoxicity and transfection efficacy. Pendent moieties have been shown to modify the structure and stability of polymer-DNA polyplexes to facilitate cellular release, with several reports exploring the effects of fine tuning a substituent alkyl chain length [23-25]. However, comparably few studies have investigated the nature of branching within an alkyl group and its effect on transfection efficacy. As our platform is amenable to a wide variety of BACCl derivatives, we synthesized two that would directly compare the branching of the alkyl chain: $n$-butyl (Bu; $\mathrm{BACBu})$ and isopropyl (iP; BACiP). Employing these ionic liquids in the post-polymerization functionalization of two polymeric backbones, poly(methylaminostyrene) (PMAS) and polyethylenimine (PEI), furnished a total of four polymers that we investigated as nonviral vectors (Figure 1). We found that there exist important synergies between the polymeric backbone and the nature of the substituent, and that the ability to simultaneously manipulate both is instrumental for the optimization of efficient nonviral gene delivery vectors.
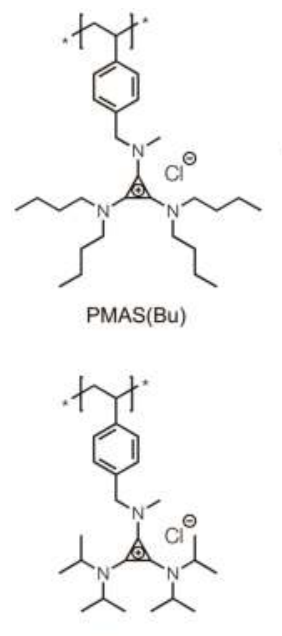

PMAS(iP)

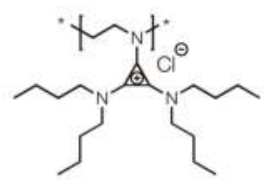

$\operatorname{PEI}(\mathrm{Bu})$

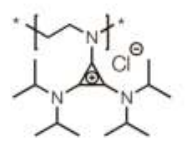

PEl(iP)

Figure 1. Trisaminocyclopropenium (TAC) polymer structures examined for biocompatibility and transfection efficacy. PEI: polyethylenimine; PMAS: poly(methylaminostyrene); Bu: $n$-butyl; iP: isopropyl.

\section{Materials and Methods}

\subsection{Materials}

All materials were purchased from Sigma-Aldrich (St. Louis, MO, USA) and were used without further purification except as noted below. Deuterated solvents used for NMR spectroscopy were 
purchased from Thermo Fisher Scientific (Waltham, MA, USA). Spectrum Labs dialysis bags were purchased from VWR (Radnor, PA, USA). Organic solutions were concentrated by use of a Buchi (New Castle, DE, USA) rotary evaporator.

\subsection{Procedure for the Synthesis of 1,2-Bis(dibutylamino)-3-chlorocyclopropenium Chloride (BACBu)}

\subsubsection{Synthesis of 2,3-Bis(dibutylamino)-1-cyclopropenone}

This procedure was performed at ambient conditions. Dibutylamine ( $24.5 \mathrm{~g}, 190 \mathrm{mmol}, 8.0$ equiv) was slowly added to a solution of pentachlorocyclopropane ( $5.0 \mathrm{~g}, 23.6 \mathrm{mmol}, 1.0$ equiv) in $\mathrm{CH}_{2} \mathrm{Cl}_{2}$ $(250 \mathrm{~mL})$ in a $1 \mathrm{~L}$ round-bottom flask at $0{ }^{\circ} \mathrm{C}$. The solution turned orange, and was allowed to warm to room temperature with stirring overnight. The reaction mixture was washed with $1 \mathrm{M}$ $\mathrm{HCl}(3 \times 100 \mathrm{~mL})$, deionized (DI) water $(1 \times 100 \mathrm{~mL})$, and brine $(1 \times 100 \mathrm{~mL})$ dried over magnesium sulfate, and concentrated in vacuo to yield a crude orange solid. The solid was dissolved in tert-butanol $(50 \mathrm{~mL})$ and to this was added potassium hydroxide $(10 \mathrm{~g}, 178 \mathrm{mmol})$ in DI water $(15 \mathrm{~mL})$. The solution was heated at $70{ }^{\circ} \mathrm{C}$ for $2 \mathrm{~h}$, and then water was removed by rotary evaporation. The resulting solid was dissolved in $\mathrm{CH}_{2} \mathrm{Cl}_{2}$ and filtered to remove salt. The organic solution was dried with anhydrous magnesium sulfate, and concentrated in vacuo to a crude yellow oil. The crude material was purified by silica gel chromatography ( $100 \%$ EtOAc; $5 \% \mathrm{MeOH}$ in $\mathrm{CH}_{2} \mathrm{Cl}_{2}$ ) to yield the title product as an orange solid (2.19 g, $7.08 \mathrm{mmol}, 30 \%$ two-step yield). ${ }^{1} \mathrm{H} \mathrm{NMR}\left(400 \mathrm{MHz}, \mathrm{CDCl}_{3}\right) \delta 3.16(\mathrm{t}, 8 \mathrm{H}$, $\left.\mathrm{NCH}_{2} \mathrm{CH}_{2} \mathrm{CH}_{2} \mathrm{CH}_{3}\right), 1.59\left(\mathrm{~m}, 8 \mathrm{H}, \mathrm{NCH}_{2} \mathrm{CH}_{2} \mathrm{CH}_{2} \mathrm{CH}_{3}\right), 1.34\left(\mathrm{~m}, 8 \mathrm{H}, \mathrm{NCH}_{2} \mathrm{CH}_{2} \mathrm{CH}_{2} \mathrm{CH}_{3}\right), 0.94(\mathrm{t}, 12 \mathrm{H}$, $\mathrm{NCH}_{2} \mathrm{CH}_{2} \mathrm{CH}_{2} \mathrm{CH}_{3}$ ).

\subsubsection{Synthesis of 1,2-Bis(dibutylamino)-3-chlorocyclopropenium Chloride (BACBu)}

Oxalyl chloride $(0.09 \mathrm{~mL}, 0.9 \mathrm{mmol}, 2.0$ equiv) was slowly added to a solution of 2,3-bis(dibutylamino)-1-cyclopropenone $\left(0.150 \mathrm{~g}, 0.45 \mathrm{mmol}, 1.0\right.$ equiv) in dry $\mathrm{CH}_{2} \mathrm{Cl}_{2}(5 \mathrm{~mL})$ at $0{ }^{\circ} \mathrm{C}$ under argon. The solution was warmed to room temperature and allowed to react for $1 \mathrm{~h}$. The solution was then concentrated in vacuo to yield the title product as a brown liquid in quantitative yield. ${ }^{1} \mathrm{H}$ NMR ( $\left.400 \mathrm{MHz}, \mathrm{CDCl}_{3}\right) \delta 3.64\left(\mathrm{t}, 4 \mathrm{H}, \mathrm{NCH}_{2} \mathrm{CH}_{2} \mathrm{CH}_{2} \mathrm{CH}_{3}\right), 3.50\left(\mathrm{t}, 4 \mathrm{H}, \mathrm{NCH}_{2} \mathrm{CH}_{2} \mathrm{CH}_{2} \mathrm{CH}_{3}\right)$, $1.76\left(\mathrm{~m}, 4 \mathrm{H}, \mathrm{NCH}_{2} \mathrm{CH}_{2} \mathrm{CH}_{2} \mathrm{CH}_{3}\right), 1.66\left(\mathrm{~m}, 4 \mathrm{H}, \mathrm{NCH}_{2} \mathrm{CH}_{2} \mathrm{CH}_{2} \mathrm{CH}_{3}\right), 1.40\left(\mathrm{~m}, 8 \mathrm{H}, \mathrm{NCH}_{2} \mathrm{CH}_{2} \mathrm{CH}_{2} \mathrm{CH}_{3}\right)$, $0.99\left(\mathrm{t}, 12 \mathrm{H}, \mathrm{NCH}_{2} \mathrm{CH}_{2} \mathrm{CH}_{2} \mathrm{CH}_{3}\right)$.

\subsection{Synthesis of $P M A S(B u)$}

The procedure was performed open to the atmosphere. Poly(methylaminostyrene) (PMAS) (50 mg, $0.3 \mathrm{mmol}$, 1 equiv, DP: 50, $M_{\mathrm{n}}$ : 7400, $M_{\mathrm{W}}: 8300$, Đ: 1.08), synthesized according to previously reported procedures [22], was dissolved in $\mathrm{CHCl}_{3}(8 \mathrm{~mL})$ in a scintillation vial equipped with a stir bar. To the vial was added $N, N$-diisopropylethylamine ( $115 \mathrm{mg}, 0.9 \mathrm{mmol}, 3$ equiv) and BACBu $\left(160 \mathrm{mg}, 0.46 \mathrm{mmol}, 1.5\right.$ equiv). The reaction mixture was allowed to stir at $65^{\circ} \mathrm{C}$ for $3 \mathrm{~h}$. The resulting solution was concentrated in vacuo, dissolved in minimum acetone and precipitated once into ethyl acetate at $-78{ }^{\circ} \mathrm{C}$. The resulting powder was dissolved in methanol and transferred to a $3.5 \mathrm{k}$ MWCO Spectrum Labs dialysis bag and dialyzed against methanol followed by concentration under vacuum to yield a pale brown powder $\left(60 \mathrm{mg}, 42 \%\right.$ yield). ${ }^{1} \mathrm{H} \mathrm{NMR}\left(500 \mathrm{MHz}, \mathrm{CDCl}_{3}\right) \delta 7.18-6.07(\mathrm{~b}, 200 \mathrm{H}$, $\mathrm{ArH}), 4.99-4.39$ (b, $\left.100 \mathrm{H}, \mathrm{ArCH}_{2} \mathrm{~N}\right), 3.78-2.91$ (b, 575H, $\mathrm{NCH}_{3}, \mathrm{NCH}_{2} \mathrm{CH}_{2} \mathrm{CH}_{2} \mathrm{CH}_{3}$ ), 1.98-1.58 (b, $400 \mathrm{H}, \mathrm{NCH}_{2} \mathrm{CH}_{2} \mathrm{CH}_{2} \mathrm{CH}_{3}$ ) 1.39-1.08 (b, 650H, $\left.\mathrm{NCH}_{2} \mathrm{CH}_{2} \mathrm{CH}_{2} \mathrm{CH}_{3}, \mathrm{ArCHCH}_{2}\right) 1.02-0.63$ (b, 600H, $\mathrm{NCH}_{2} \mathrm{CH}_{2} \mathrm{CH}_{2} \mathrm{CH}_{3}$ ).

\subsection{Synthesis of PEI(Bu)}

This procedure was performed open to the atmosphere. Linear $25 \mathrm{k}$ polyethyleneimine $(20 \mathrm{mg}$, $0.46 \mathrm{mmol}, 1$ equiv) was dissolved in $\mathrm{CHCl}_{3}(8 \mathrm{~mL})$ in a scintillation vial equipped with a stir bar. To the vial was added $N, N$-diisopropylethylamine $(180 \mathrm{mg}, 1.4 \mathrm{mmol}, 3$ equiv) and BACBu $(250 \mathrm{mg}$, $0.70 \mathrm{mmol}, 1.5$ equiv). The reaction mixture was allowed to stir at $65^{\circ} \mathrm{C}$ for $3 \mathrm{~h}$. The resulting solution 
was concentrated in vacuo and precipitated once into ethyl acetate at $-78^{\circ} \mathrm{C}$. The resulting powder was dissolved in methanol and transferred to a 3.5k MWCO Spectrum Labs dialysis bag and dialyzed against methanol. The resulting solution was concentrated vacuum to yield a yellow-brown powder (25 mg, 15\% yield). ${ }^{1} \mathrm{H}$ NMR (400 MHz, $\left.\mathrm{CDCl}_{3}\right) \delta 4.45-3.15\left(\mathrm{~b}, 7000 \mathrm{H}, \mathrm{CH}_{2} \mathrm{CH}_{2} \mathrm{~N}\right.$ ) $\left.581, \mathrm{NCH}_{2} \mathrm{CH}_{2} \mathrm{CH}_{2} \mathrm{CH}_{3}\right)$ 1.71-1.55 (b, 3500H, NCH $\mathrm{CH}_{2} \mathrm{CH}_{2} \mathrm{CH}_{3}$ ), 1.43-1.22 (b, 5400H, $\left.\mathrm{NCH}_{2} \mathrm{CH}_{2} \mathrm{CH}_{2} \mathrm{CH}_{3}\right), 1.00-0.90$ (b, 6200H, $\mathrm{NCH}_{2} \mathrm{CH}_{2} \mathrm{CH}_{2} \mathrm{CH}_{3}$ ).

2.5. Synthesis of 1,2-Bis(diisopropylamino)-3-chlorocyclopropenium Chloride (BACiP), PMAS(iP), and PEI(iP)

The preparations of $\mathrm{BAC}(\mathrm{iP})$ and the subsequently derivatized polymers have been previously reported $[22,26]$.

\subsection{Cell Culture}

HEK-293T cells (American Type Culture Collection, Manassas, VA, USA) were grown in Dulbecco's Modified Eagle Medium (DMEM) with L-glutamine (Gibco, Grand Island, NY, USA) supplemented with 10\% fetal bovine serum (FBS) (Atlanta Biologicals, Flowery Branch, GA, USA) and $1 \%$ penicillin/streptomycin (Gibco, Grand Island, NY, USA). Cultures were incubated in humidified tissue incubators at $37^{\circ} \mathrm{C}$ and $5 \% \mathrm{CO}_{2}$.

\subsection{Cell Viability}

Trypan blue dye exclusion cell counting was performed in triplicate with an automated cell counter (ViCell, Beckman-Coulter, Brea, CA, USA). Cell viability under experimental conditions is reported as a percentage relative to untreated cells.

\subsection{Cell Transfection and Luciferase Expression}

HEK-293T cells were seeded on 12-well plates at a density of $5 \times 10^{4}$ cells/well $24 \mathrm{~h}$ prior to transfection. The media was then evacuated, replaced with fresh, and supplemented with polymer-pDNA polyplexes. Polyplexes were prepared by adding polymer solutions in RNase-free water to $3 \mu \mathrm{g}$ of plasmid DNA (pDNA) (gWiz-Luciferase, Aldevron, Fargo, ND, USA) at indicated loadings, and vortexing at $1500 \mathrm{rpm}$ for $3 \mathrm{~min}$ at room temperature. After $48 \mathrm{~h}$ of incubation, cell viability was measured, and cells were re-plated on 96-well plates at a density of $5 \times 10^{3}$ cells/well. After $24 \mathrm{~h}$ of incubation, cells were analyzed for luciferase activity according to the manufacturer's protocol. Briefly, cells were rinsed with phosphate-buffered saline (PBS) and lysed with $20 \mu \mathrm{L} /$ well $1 \times$ Cell Lysis Buffer (Promega, Madison, WI, USA). To the cell lysates was added $100 \mu \mathrm{L} /$ well of Luciferase Assay Reagent (Promega) and the light produced was measured immediately on a plate reader (PerkinElmer, Waltham, MA). Results were expressed as relative light units (RLU) normalized to cell counts, with error bars showing the standard deviation of triplicate measurement.

\subsection{Hydrodynamic Size and Zeta Potential Measurement}

Polyplex size and zeta potential were measured on a Malvern Zetasizer Nano ZS (Malvern Instruments, Malvern, UK). For all measurements, polyplexes were diluted 1:100 in Milli-Q water at neutral $\mathrm{pH}$. The reported diameters are the average of three measurements, where each measurement comprises at least 10 acquisitions, and the zeta potential was calculated according to the Smoluchowski approximation.

\subsection{Gel Electrophoresis Shift Assay}

Polyplexes were prepared at different weight ratios by adding $10 \mu \mathrm{L}$ of polymer in Milli-Q $\mathrm{H}_{2} \mathrm{O}$ to $10 \mu \mathrm{L}$ of pDNA $(5 \mathrm{ng} / \mu \mathrm{L})$, and vortexing at $1500 \mathrm{rpm}$ for $3 \mathrm{~min}$ at room temperature. To the polyplex solution was then added $2 \mu \mathrm{L}$ of loading dye, for a total volume of $22 \mu \mathrm{L}$, which was subsequently 
added to the well. Agarose gels were prepared as $1 \mathrm{wt} \%$ in tris-acetate EDTA (TAE) buffer with $2 \mu \mathrm{L}$ ethidium bromide and run at $100 \mathrm{~V}$ for $20 \mathrm{~min}$. Gels were visualized under UV illumination at $365 \mathrm{~nm}$.

\section{Results and Discussion}

\subsection{Cationic Polymer Synthesis}

The candidate nonviral vectors were synthesized from neutral parent precursor polymers in a post-polymerization functionalization strategy. For this study, we employed two parent polymers-PMAS $\left(7.5 \mathrm{~kg} \cdot \mathrm{mol}^{-1}\right)$, synthesized according to literature [22], and commercially available PEI (25 kg. mol ${ }^{-1}$; linear) — to compare the effects of backbone structure. Both were subsequently transformed into TAC derivatives by reaction with a BACCl salt, in a "click" conjugation reaction proceeding under mild conditions with stoichiometric amounts of reactants. The cationic nature of cyclopropenium-based polymers prevents their characterization by gel permeation chromatography [26], so PMAS was shown to have a narrow dispersity less than 1.1 (Supplementary Materials, Figure S1), and complete functionalization was confirmed by NMR.

We elected to study two BACCl structures comprising dialkylamino substituents differing in the degree of branching, and thereby "floppiness", as well as hydrophobicity, as the Bu-derivatized polymers have one more carbon. Complete functionalization of both BAC(Bu), containing n-butyl substituents, and BAC(iP), containing isopropyl substituents, was confirmed by proton nuclear magnetic resonance spectroscopy. Quantitatively functionalizing polymers holds effects of dispersity and degree of polymerization constant, permitting direct comparisons of subtle structural changes on macromolecular properties.

\subsection{Biocompatibility Studies}

Careful engineering of cationic polymers is necessary to enable permeation of the cell membrane without reducing overall viability. Studies have shown that cationic polymers form pores in the cell membrane to mediate entry, but they also reduce overall cell viability [27]. Pore formation typically involves intercalation of the cell membrane's lipid bilayer by aliphatic groups on the delivery agent; thus, tuning TAC's alkyl substituents represents an opportunity to promote cell entry while minimizing cell death associated with membrane destabilization. Therefore, to probe the impact of alkyl chain conformation on cell viability and transfection efficiency, the series of TAC-functionalized polymers were assessed and compared as vectors via cytotoxicity assays and luciferase transfection experiments in HEK-293T cells.

All four homopolymers were highly water-soluble, permitting their condensation with an aqueous solution of plasmid DNA (pDNA) containing the firefly luciferase reporter gene. Combining the polymers at varied loadings with a fixed amount of pDNA, and subsequently incubating in cells for $2 \mathrm{~d}$, revealed the polymers' biocompatibility as a function of loading. We found that PEI(iP) and PMAS(Bu) were the most biocompatible with high cell viabilities through loadings of $20 \mu \mathrm{g} \cdot \mathrm{mL}^{-1}$ (Figure 2). Surprisingly, their counterparts, PEI(Bu) and PMAS(iP), exhibited notable toxicity at all loadings tested. All TAC-derived polymers here were highly toxic at loadings of $50 \mu \mathrm{g} \cdot \mathrm{mL}^{-1}$ and greater, similar to linear PEI. This stands in contrast to our previous work, where polymers with more rigid amino substituents on the TAC ion were still viable in this regime [22]. Thus, rigidity or flexibility of substituent chains stand as an important parameter to understand for optimal gene transfection. These results suggest there is a complex interaction between a polymer backbone and its substituent in the design of biocompatible gene delivery vectors. 


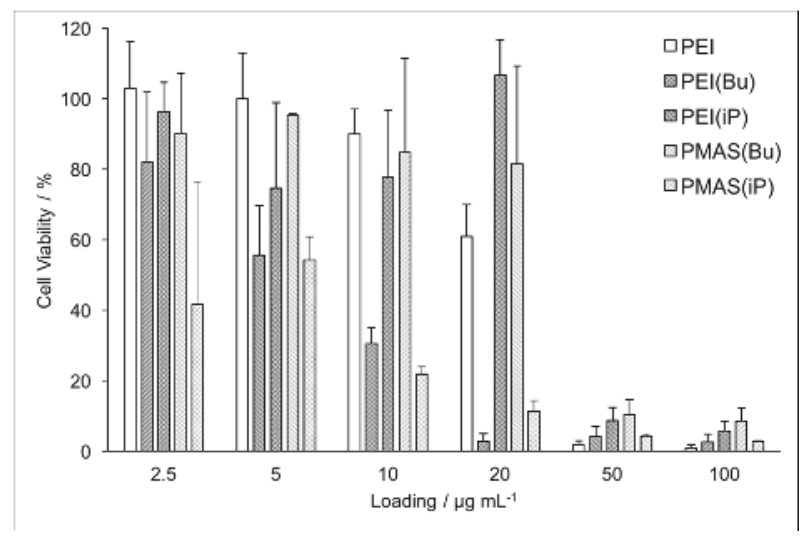

Figure 2. Biocompatibility of TAC-based polymers at various doses in HEK-293T cells following $48 \mathrm{~h}$ incubation. Viability is measured by trypan blue dye exclusion and normalized to untreated cells. Error bars show the standard deviation of triplicate measurement.

\subsection{DNA Binding and In Vitro Gene Transfection}

In order to affect transfection, candidate gene delivery vectors must condense nucleic acids into a polyplex, permeate the cell membrane, escape the endosome, and release their payload [27]. We found that fine tuning the substituent chain branching had a dramatic influence on delivery efficacy. In order to assess the amount of polymer necessary to completely condense pDNA into a polyplex, gel electrophoresis shift assays were performed (Figures S2-S5). While all polymers were able to fully bind the pDNA by a weight ratio of 3:33:1, PEI(iP) was the most efficient, binding at a weight ratio of only $0.83: 1$. This corresponds to the lowest polymer loading tested for either biocompatibility or transfection, and less than 1 TAC unit per phosphate anion of pDNA (Tables S1 and S2). However, binding efficiency is not a clear indicator of delivery efficiency, as too favorable an interaction can be detrimental for eventual release of the genetic material.

Luciferase expression assays revealed a significant dependence on the nature of the amino substituent and polymer backbone for successful gene delivery. While all TAC-based polymers transfected pDNA significantly better than the untreated controls, PMAS(iP) demonstrated the highest transfection efficacy (Figure 3). As is the case with unmodified linear PEI, successful delivery of intact pDNA to cells comes at the cost of significant cytotoxicity. By contrast, the nontoxic PEI(iP) demonstrated a much lower luciferase activity. Interestingly, PEI(iP) was the most efficient at compacting pDNA into a polyplex, suggesting that it binds nucleic acids too strongly and never releases its payload. Converting either of the polymer backbones into a TAC bearing n-butyl chains seemed to yield successful nonviral vectors capable of both binding and slowly releasing pDNA. This could potentially be attributed to critical destabilization of the cell and endosomal membranes due to the long, flexible alkyl substituents. As none of the TAC polymers examined here contain any protonatable centers, it is unlikely that polyplexes escape the endosome via a proton sponge mechanism [24]. At their optimal loadings, both $\mathrm{PEI}(\mathrm{Bu})$ and $\mathrm{PMAS}(\mathrm{Bu})$ demonstrated two orders-of-magnitude improvement over untreated control cells. Taken together with the cytotoxicity and pDNA-binding data, we conclude that amongst this family of TAC polymers, $\operatorname{PMAS}(\mathrm{Bu})$ is the most potent nonviral vector. 


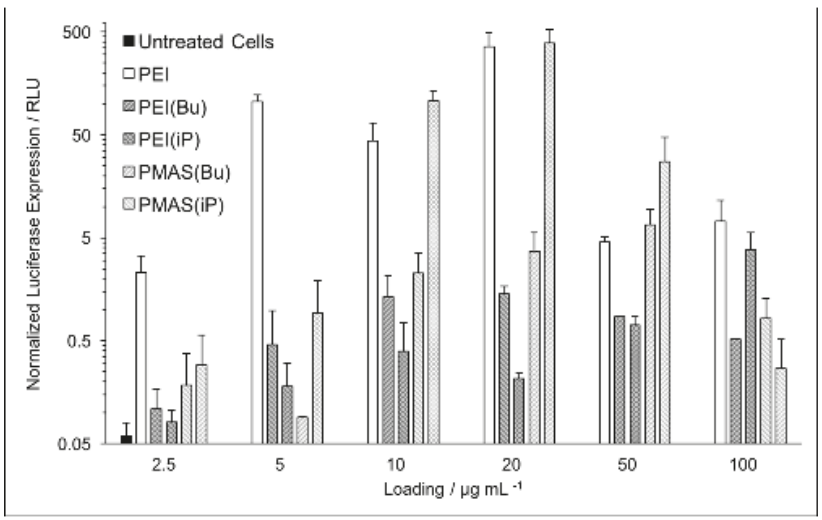

Figure 3. Luciferase expression in HEK-293T cells transfected with pDNA containing the firefly luciferase reporter gene using TAC polymers. Luciferase expression is measured after $48 \mathrm{~h}$ incubation with specified polymer loadings (all with pDNA loadings of $3 \mu \mathrm{g} \cdot \mathrm{mL}^{-1}$ ) and normalized by cell count. Error bars show the standard deviation of triplicate measurement.

\subsection{Hydrodynamic Size and Zeta Potential Measurements}

Dynamic light scattering determined the hydrodynamic diameter $\left(D_{H}\right)$ and zeta potential $(\zeta)$ of the polyplexes at their optimal loading for transfection efficacy (Table 1). All four cationic polymers formed stable polyplexes of small sizes and highly positive charge. The polyplexes all exhibit a hydrodynamic diameter in the size regime considered optimal for successful gene transfection. Notably, the polymers modified with BAC(iP) resulted in more positively charged polyplexes than those with $\mathrm{BAC}(\mathrm{Bu})$, which could be a result of enhanced hydrophobic screening of the charge by the longer, flexible n-butyl chains $[20,28]$. These data suggest the dramatic differences in cytotoxicity and transfection efficacy between the four TAC-based polymers are likely a result of structural variations rather than significant size or surface charge differences.

Table 1. Characterization of transfection agents and polyplexes at optimal transfection efficacy.

\begin{tabular}{ccccc}
\hline Transfection agent $^{\mathbf{1}}$ & $\mathbf{M M}^{\mathbf{2}} \mathbf{( k D a )}$ & Charge Ratio $^{\mathbf{3}}$ & $\mathbf{D}_{\mathbf{H}}(\mathbf{n m})$ & $\zeta_{\text {Potential }}(\mathbf{m V})$ \\
\hline PEI(Bu) & 215 & $6: 1$ & $110 \pm 40$ & $45 \pm 7$ \\
PEI(iP) & 182 & $35: 1$ & $100 \pm 40$ & $58 \pm 5$ \\
PMAS(Bu) & 25 & $12: 1$ & $150 \pm 50$ & $31 \pm 8$ \\
PMAS(iP) & 21 & $5: 1$ & $160 \pm 40$ & $44 \pm 6$ \\
\hline
\end{tabular}

1 Polyplexes of polymers at the loading corresponding to highest transfection efficacy in Figure $3{ }^{2}$ Molecular mass of transfection agent, calculated based on commercial linear 25k PEI; for PMAS(R) materials, PMAS was measured by gel permeation chromatography (GPC) calibrated using polystyrene (PS) standards of narrow dispersity, then calculated for the corresponding TAC group. ${ }^{3}$ Ratio of TAC to phosphate anions.

\section{Conclusions}

We have shown that fine-tuning polymer structure can have dramatic effects on macromolecular properties-in particular, cytotoxicity and gene delivery. Beginning with two discrete parent polymers, we synthesized two pairs of TAC-based polymers differing solely in alkyl substituent identity, branching, and polymer backbone. While none of the examined polymers surpass linear PEI in transfection efficiency, our results demonstrate that there is an important interplay between polymer backbone and substituent structure, and that both must be carefully considered in the design of nonviral vectors. Amongst our examined TAC-based polymers, PMAS(Bu) exhibited the best balance of biocompatibility, efficient DNA binding, and transfection efficacy. While hydrophobic modifications 
of nonviral vectors are frequently reported to promote transfection, our results demonstrate that a careful balance of hydrophobicity and substituent flexibility must be achieved for optimal gene delivery. Importantly, this work exemplifies that design of transfection reagents demands precise control over all aspects of polymer structure and a robust, modular platform from which to study them. Only through these kinds of systematic studies can an optimized nonviral vector to achieve successful gene delivery be developed.

Supplementary Materials: The following are available online at www.mdpi.com/2073-4360/9/3/79/s1, Figure S1: GPC trace of poly(methylaminostyrene), the parent polymer for both PMAS(Bu) and PMAS(iP); Figure S2: Gel electrophoresis shift assay of pDNA polyplexes formed with PMAS(Bu) at the indicated polymer: pDNA weight ratios. All pDNA is bound in polyplexes at a weight ratio of 1.66 PMAS(Bu): 1 pDNA; Figure S3: Gel electrophoresis shift assay of pDNA polyplexes formed with $\mathrm{PEI}(\mathrm{Bu})$ at the indicated polymer:pDNA weight ratios. All pDNA is bound in polyplexes at a weight ratio of $3.33 \mathrm{PEI}(\mathrm{Bu}): 1 \mathrm{pDNA}$; Figure S4: Gel electrophoresis shift assay of pDNA polyplexes formed with PMAS(iP) at the indicated polymer:pDNA weight ratios. All pDNA is bound in polyplexes at a weight ratio of 1.66 PMAS(iP): 1 pDNA; Figure S5: Gel electrophoresis shift assay of pDNA polyplexes formed with PEI(iP) at the indicated polymer:pDNA weight ratios. All pDNA is bound in polyplexes at a weight ratio of $0.83 \mathrm{PEI}(\mathrm{iP})$ : 1 pDNA; Table S1: Polymer loading and pDNA weight ratios for gel electrophoresis and transfection experiments; Table S2: Conversion of weight ratios to charge ratios for each tested polymer.

Acknowledgments: This work was funded by the National Science Foundation (NSF CAREER DMR-1351293, and CHE 1464992), ACS Petroleum Research Fund, and 3M Non-Tenured Faculty Award. S.D.B. is grateful for NSF GRFP (DGE-16-44869). We are grateful to the Stockwell lab for use of their cell culture hoods and plate reader.

Author Contributions: Spencer D. Brucks, Jessica L. Freyer, Tristan H. Lambert, and Luis M. Campos conceived and designed the experiments; Spencer D. Brucks and Jessica L. Freyer performed the experiments; The manuscript was written by Spencer D. Brucks, Jessica L. Freyer, and Luis M. Campos, with contributions from all authors.

Conflicts of Interest: The authors declare no conflict of interest.

\section{References}

1. Yin, H.; Kanasty, R.L.; Eltoukhy, A.A.; Vegas, A.J.; Dorkin, J.R.; Anderson, D.G. Non-viral vectors for gene-based therapy. Nat. Rev. Genet. 2014, 15, 541-555. [CrossRef] [PubMed]

2. Mintzer, M.A.; Simanek, E.E. Nonviral vectors for gene delivery. Chem. Rev. 2009, 109, 259-302. [CrossRef] [PubMed]

3. Bender, E. Gene therapy: Industrial strength. Nature 2016, 537, S57-S59. [CrossRef] [PubMed]

4. Li, S.D.; Huang, L. Non-viral is superior to viral gene delivery. J. Control. Release 2007, 3, 181-183. [CrossRef] [PubMed]

5. Kay, M.A. State-of-the-art gene-based therapies: The road ahead. Nat. Rev. Genet. 2011, 5, 316-328. [CrossRef] [PubMed]

6. Kostarelos, K.; Miller, A.D. Synthetic, self-assembly ABCD nanoparticles: A structural paradigm for viable synthetic non-viral vectors. Chem. Soc. Rev. 2005, 34, 970-994. [CrossRef] [PubMed]

7. Samal, S.K.; Dash, M.; Van Vlierberghe, S.; Kaplan, D.L.; Chiellini, E.; van Blitterswijk, C.; Moroni, L.; Dubruel, P. Cationic polymers and their therapeutic potential. Chem. Soc. Rev. 2012, 21, 7147-7194. [CrossRef] [PubMed]

8. Merdan, T.; Kopeček, J.; Kissel, T. Prospects for cationic polymers in gene and oligonucleotide therapy against cancer. Adv. Drug Deliv. Rev. 2002, 54, 715-758. [CrossRef]

9. Boussif, O.; Lezoualc'h, F.; Zanta, M.A.; Mergny, M.D.; Scherman, D.; Demeneix, B.; Behr, J.P. A versatile vector for gene and oligonucleotide transfer into cells in culture and in vivo: Polyethylenimine. Proc. Natl. Acad. Sci. USA 1995, 92, 7297-7301. [CrossRef] [PubMed]

10. Godbey, W.T.; Wu, K.K.; Mikos, A.G. Size matters: Molecular weight affects the efficiency of poly(ethylenimine) as a gene delivery vehicle. J. Biomed. Mater. Res. 1998, 3, 268-275. [CrossRef]

11. Fischer, D.; Bieber, T.; Li, Y.; Elsässer, H.P.; Kissel, T. A novel non-viral vector for DNA delivery based on low molecular weight, branched polyethylenimine: Effect of molecular weight on transfection efficiency and cytotoxicity. Pharm. Res. 1999, 8, 1273-1279. [CrossRef]

12. Layman, J.M.; Ramirez, S.M.; Green, M.D.; Long, T.E. Influence of polycation molecular weight on poly(2-dimethylaminoethyl methacrylate)-mediated DNA delivery in vitro. Biomacromolecules 2009, 10, 1244-1252. [CrossRef] [PubMed] 
13. Bishop, C.J.; Abubaker-Sharif, B.; Guiriba, T.; Tzeng, S.Y.; Green, J.J. Gene delivery polymer structure-function relationships elucidated via principal component analysis. Chem. Commun. 2015, 51, 12134-12137. [CrossRef] [PubMed]

14. Breitenkamp, R.B.; Emrick, T. Pentalysine-grafted ROMP polymers for DNA complexation and delivery. Biomacromolecules 2008, 9, 2495-2500. [CrossRef] [PubMed]

15. Synatschke, C.V.; Schallon, A.; Jérôme, V.; Freitag, R.; Müller, A.H.E. Influence of polymer architecture and molecular weight of poly(2-(dimethylamino)ethyl methacrylate) polycations on transfection efficiency and cell viability in gene delivery. Biomacromolecules 2011, 12, 4247-4255. [CrossRef] [PubMed]

16. Raup, A.; Stahlschmidt, U.; Jérôme, V.; Synatschke, C.V.; Müller, A.H.E.; Freitag, R. Influence of polyplex formation on the performance of star-shaped polycationic transfection agents for mammalian cells. Polymers 2016, 8, 224. [CrossRef]

17. Zhou, D.; Cutlar, L.; Gao, Y.; Wang, W.; O'Keeffe-Ahern, J.; McMahon, S.; Duarte, B.; Larcher, F.; Rodriguez, B.J.; Greiser, U.; et al. The transition from linear to highly branched poly( $\beta$-amino ester)s: Branching matters for gene delivery. Sci. Adv. 2016, 2, 1-15. [CrossRef] [PubMed]

18. Cutlar, L.; Zhou, D.; Gao, Y.; Zhao, T.; Greiser, U.; Wang, W.; Wang, W. Highly branched poly( $\beta$-amino esters): Synthesis and application in gene delivery. Biomacromolecules 2015, 16, 2609-2617. [CrossRef] [PubMed]

19. Zhou, D.; Gao, Y.; O'Keeffe Ahern, J.; Xu, Q.; Huang, X.; Greiser, U.; Wang, W. Development of branched poly(5-amino-1-pentanol-co-1,4-butanediol diacrylate) with high gene transfection potency across diverse cell types. ACS Appl. Mater. Interfaces 2016, 8, 34218-34226. [CrossRef] [PubMed]

20. Zhou, D.; Gao, Y.; Aied, A.; Cutlar, L.; Igoucheva, O.; Newland, B.; Alexeeve, V.; Greiser, U.; Uitto, J.; Wang, W. Highly branched poly( $\beta$-amino ester)s for skin gene therapy. J. Control. Release 2016, 244, 336-346. [CrossRef] [PubMed]

21. Liu, S.; Gao, Y.; Zhou, D.; Greiser, U.; Guo, T.; Guo, R.; Wang, W. Biodegradable highly branched poly( $\beta$-amino ester)s for targeted cancer cell gene transfection. ACS Biomater. Sci. Eng. 2016, 18, 440-451. [CrossRef]

22. Freyer, J.L.; Brucks, S.D.; Gobieski, G.S.; Russell, S.T.; Yozwiak, C.E.; Sun, M.; Chen, Z.; Jiang, Y.; Bandar, J.S.; Stockwell, B.R.; et al. Clickable poly(ionic liquids): A materials platform for transfection. Angew. Chem. Int. Ed. 2016, 55, 12382-12386. [CrossRef] [PubMed]

23. Gao, Y.; Chen, L.; Zhang, Z.; Gu, W.; Li, Y. Linear cationic click polymer for gene delivery: Synthesis, biocompatibility, and in vitro transfection. Biomacromolecules 2010, 11, 3102-3111. [CrossRef] [PubMed]

24. Hemp, S.T.; Allen, M.H., Jr.; Green, M.D.; Long, T.E. Phosphonium-containing polyelectrolytes for nonviral gene delivery. Biomacromolecules 2012, 13, 231-238. [CrossRef] [PubMed]

25. Jarzębińska, A.; Pasewald, T.; Lambrecht, J.; Mykhaylyk, O.; Kümmerling, L.; Beck, P.; Hasenpusch, G.; Rudolph, C.; Plank, C.; Dohmen, C. A Single methylene group in oligoalkylamine-based cationic polymers and lipids promotes enhanced mRNA delivery. Angew. Chem. Int. Ed. 2016, 55, 9591-9595. [CrossRef] [PubMed]

26. Jiang, Y.; Freyer, J.L.; Cotanda, P.; Brucks, S.D.; Killops, K.L.; Bandar, J.S.; Torsitano, C.; Balsara, N.P.; Lambert, T.H.; Campos, L.M. The evolution of cyclopropenium ions into functional polyelectrolytes. Nat. Commun. 2015, 6, 5950. [CrossRef] [PubMed]

27. Vaidyanathan, S.; Orr, B.G.; Banaszak Holl, M.M. Role of cell membrane-vector interactions in successful gene delivery. Acc. Chem. Res. 2016, 49, 1486-1493. [CrossRef] [PubMed]

28. Santos, J.L.; Oliveira, H.; Pandita, D.; Rodrigues, J.; Pêgo, A.P.; Granja, P.L.; Tomás, H. Functionalization of poly(amidoamine) dendrimers with hydrophobic chains for improved gene delivery in mesenchymal stem cells. J. Control. Release 2010, 144, 55-64. [CrossRef] [PubMed] 
Article

\title{
Influences of Alkyl and Aryl Substituents on Iminopyridine Fe(II)- and Co(II)-Catalyzed Isoprene Polymerization
}

\author{
Lihua Guo ${ }^{1}$, Xinyu Jing ${ }^{1}$, Shuoyan Xiong ${ }^{2}$, Wenjing Liu ${ }^{1}$, Yanlan Liu ${ }^{1}$, Zhe Liu ${ }^{1}$ and \\ Changle Chen ${ }^{2, *}$ \\ 1 School of Chemistry and Chemical Engineering, Qufu Normal University, Qufu 273165, China; \\ 18753770200@163.com (L.G.); m18463758798@163.com (X.J.); liuwenjingaaa@126.com (W.L.); \\ lyl373740@163.com (Y.L.); liuzheqd@163.com (Z.L.) \\ 2 Key Laboratory of Soft Matter Chemistry, Chinese Academy of Sciences, \\ Department of Polymer Science and Engineering, University of Science and Technology of China, \\ Hefei 230026, China; xiong18@mail.ustc.edu.cn \\ * Correspondence: changle@ustc.edu.cn; Tel.: +86-551-6360-1495
}

Academic Editor: Alexander Böker

Received: 28 September 2016; Accepted: 28 October 2016; Published: 3 November 2016

\begin{abstract}
A series of alkyl- and aryl-substituted iminopyridine Fe(II) complexes 1a-7a and Co(II) complexes $\mathbf{2 b}, \mathbf{3 b}, \mathbf{5 b}$, and $\mathbf{6 b}$ were synthesized. The activator effect, influence of temperature, and, particularly, the alkyl and aryl substituents' effect on catalytic activity, polymer molecular weight, and regio-/stereoselectivity were investigated when these complexes were applied in isoprene polymerization. All of the Fe(II) complexes afforded polyisoprene with high molecular weight and moderate cis-1,4 selectivity. In contrast, the Co(II) complexes produced polymers with low molecular weight and relatively high cis-1,4 selectivity. In the iminopyridine $\mathrm{Fe}(\mathrm{II})$ system, the alkyl and aryl substituents' effect exhibits significant variation on the isoprene polymerization. In the iminopyridine Co(II) system, there is little influence observed on isoprene polymerization by alkyl and aryl substituents.
\end{abstract}

Keywords: iminopyridine; Iron(II); Cobalt(II); isoprene polymerization; selectivity

\section{Introduction}

The polymerization of isoprene can afford polymers with various regio- and/or stereoregularities such as isotactic or syndiotactic polyisoprene via 1,2 or 3,4 addition, and cis- or trans-1,4 polyisoprene via 1,4 addition. The structures of polyisoprene strongly influence the properties of the resulting material. For example, the properties of cis-1,4 polyisoprene is very similar to those of natural rubber [1], while the properties of trans-1,4 polyisoprene is very close to those of gutta-percha [2]. The development of highly efficient and highly regio- and stereoselective catalysts plays a key role in the field of metal-catalyzed polymerization of conjugate dienes [3]. Titanium and rare-earth metal catalysts can afford cis-1,4 and trans-1,4 polybutadienes and polyisoprenes with up to $98 \%$ selectivity [4-15]. In addition, some late transition-metal catalytic systems were successfully applied in olefins [16-34], butadiene $[4,35-45]$, and isoprene $[36,37,46-50]$ polymerization. Late transition-metal catalysts have lower Lewis acid characteristics and may possess high tolerance towards functional groups and polar additives. Special attention was paid to low-cost and earth-abundant iron- and cobalt-based catalysts with well-defined molecular structures that could be easily prepared.

Recently, Dai et al. [41] showed that an aryl-substituted iminopyridine Co(II) catalyst exhibited high catalytic activity and cis-1,4-selectivity for 1,3-butadiene polymerization. Raynaud et al. [51] 
reported that the combination of the iminopyridine $\mathrm{Fe}(\mathrm{II})$ complexes, alkylaluminum, and dealkylating reagent $\left[\mathrm{Ph}_{3} \mathrm{C}\right]\left[\mathrm{B}\left(\mathrm{C}_{6} \mathrm{~F}_{5}\right)_{4}\right]$ can polymerize isoprene with high stereoselectivity. The octyl-substituted imines favor trans-1,4 insertion, whereas supermesityl-substituted imines favor cis-1,4 insertion. The authors suggested that higher electron density at the iron center may increase the trans-1,4 selectivity. However, this accidental discovery and studies of only these two catalysts make it difficult draw any rational conclusions.

Inspired by these works, we became very interested in the influence of iminopyridine ligand substituents on the selectivity of isoprene polymerization. In this work, various alkyl- and aryl-substituted iminopyridine $\mathrm{Fe}(\mathrm{II})$ and $\mathrm{Co}$ (II) complexes were synthesized and employed in isoprene polymerization when activated using an alkylaluminum (methylaluminoxane (MAO) or $\mathrm{AlEtCl}_{2}$ ) (Scheme 1). The effects of the imine moiety on the catalytic activity, molecular weight, and, particularly, the regio- and stereoselectivity were investigated.

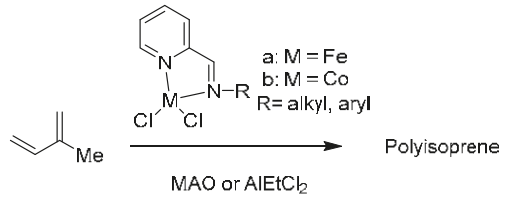

Scheme 1. Alkyl- and aryl-substituted iminopyridine Fe(II) and Co(II) complexes for isoprene polymerization.

\section{Experimental Section}

\subsection{General Information}

All manipulations of air-and-moisture sensitive materials were performed under a dry nitrogen atmosphere by using standard Schlenk techniques. Nitrogen was purified by passing through a $\mathrm{MnO}$ oxygen-removal column and an activated $4 \AA$ molecular sieve column. ${ }^{1} \mathrm{H}$ and ${ }^{13} \mathrm{C} \mathrm{NMR}$ spectra were recorded using $\mathrm{CDCl}_{3}$ as solvent on a Bruker Ascend ${ }^{\mathrm{TM}} 500$ spectrometer (Bruker, Karlsruhe, Germany) at room temperature unless otherwise stated. The chemical shifts of the ${ }^{1} \mathrm{H}$ and ${ }^{13} \mathrm{C}$ NMR spectra (Bruker, Karlsruhe, Germany) were referenced to tetramethylsilane (TMS). Coupling constants are in units of hertz. Fourier-transform infrared (FTIR) spectrometry was performed on a Thermo Scientific Nicolet iS5 (Thermo Fisher Scientific Corporation, Waltham, MA, USA) using the conventional $\mathrm{KBr}$ wafer technique. Elemental analysis was performed by the Analytical Center of the University of Science and Technology of China (Hefei, China). Mass spectra were recorded on a P-SIMS-Gly of Bruker Daltonics Inc. (EI, Bruker Daltonics Inc., Billerica, MA, USA). X-ray Diffraction data were collected at 298(2) K on a Bruker Smart CCD area detector (Bruker, Karlsruhe, Germany) with graphite-monochromated MoK $\alpha$ radiation $(\lambda=0.71073 \AA)$. Molecular weights and molecular weight distributions were determined by gel permeation chromatography (GPC, Waters, Milford, MA, USA) employing a series of two linear Styragel columns (HR2 and HR4) at an oven temperature of $45^{\circ} \mathrm{C}$. A Waters 1515 pump and Waters 2414 differential refractive index detector $\left(30^{\circ} \mathrm{C}\right)$ were used. The eluent was tetrahydrofuran (THF) at a flow rate of $1.0 \mathrm{~mL} \cdot \mathrm{min}^{-1}$. A series of low-polydispersity polystyrene standards was used for calibration. Hexane (Tianjin Fuyu Fine Chemical Limited Company, Tianjin, China), toluene (Laiyang Fine Chemical Factory, Laiyang, China) and THF (tetrahydrofuran, Tianjin Fuyu Fine Chemical Limited Company, Tianjin, China) were refluxed over sodium benzophenone ketyl until the solution turned blue and then distilled before use. $\mathrm{CH}_{2} \mathrm{Cl}_{2}$ (Tianjin Fuyu Fine Chemical Limited Company, Tianjin, China) was refluxed over phosphorus pentoxide for $8 \mathrm{~h}$ and distilled under a nitrogen atmosphere. Isoprene (Aladdin Industrial Corporation, Shanghai, China) was dried over $\mathrm{CaH}_{2}$ prior to use in polymerization. Ligands $\mathbf{L} 2$ and $\mathbf{L} 5$ - $\mathbf{L} 7$ were prepared according to reported procedure [51-53]. Complexes $\mathbf{2 a}, \mathbf{5} \mathbf{a}, \mathbf{6} \mathbf{a}$, and $\mathbf{6} \mathbf{b}$ were synthesized according the reported method $[41,51,54]$. All other reagents were purchased from commercial sources and used without purification. 


\subsection{General Procedure for the Synthesis of Ligands L1, L3, and $\mathbf{L 4}$}

A solution of the corresponding amine $(30 \mathrm{mmol})$ in methanol $(30 \mathrm{~mL})$ was added to pyridine-2-carbaldehyde $(30 \mathrm{mmol})$ and a drop of formic acid was subsequently added. The mixture was stirred at room temperature overnight.

Cyclohexyl(pyridin-2-yl-methylene)amine (L1): the reaction mixture was concentrated under reduced pressure. The residue was purified by distillation under vacuum to give the colorless oil. Yield: $5.37 \mathrm{~g}$ (95.1\%). ${ }^{1} \mathrm{H}$ NMR $\left(500 \mathrm{MHz}, \mathrm{CDCl}_{3}\right) \delta 8.63(d, J=3.9 \mathrm{~Hz}, 1 \mathrm{H}), 8.40(\mathrm{~s}, 1 \mathrm{H}, \mathrm{CH}=\mathrm{N}), 7.99(d, J=7.8 \mathrm{~Hz}$, $1 \mathrm{H}), 7.70(d d, J=10.6,4.1 \mathrm{~Hz}, 1 \mathrm{H}), 7.43-7.13(m, 1 \mathrm{H}), 3.53-3.19(m, 1 \mathrm{H}, \mathrm{N}-\mathrm{CH}), 2.05-1.52(m, 7 \mathrm{H})$, 1.53-1.04 (m,3H). ${ }^{13} \mathrm{C}$ NMR $\left(126 \mathrm{MHz}_{2} \mathrm{CDCl}_{3}\right) \delta 157.97(\mathrm{CH}=\mathrm{N}), 153.79,147.76,134.52,122.80,119.47$, 67.83, 32.84, 24.30, 23.13. Anal. calcd. for $\mathrm{C}_{12} \mathrm{H}_{16} \mathrm{~N}_{2}$ : C, 76.55; H, 8.57; N, 14.88; found: C, 76.13; $\mathrm{H}, 8.44$; $\mathrm{N}, 14.79$.

Adamantyl(pyridin-2-yl-methylene)amine (L3): the reaction mixture was concentrated under reduced pressure. The residue was purified by distillation under vacuum to give the light-yellow oil which quickly changed to solid at room temperature. Yield: $6.76 \mathrm{~g}(93.7 \%) .{ }^{1} \mathrm{H} \mathrm{NMR}\left(500 \mathrm{MHz}, \mathrm{CDCl}_{3}\right) \delta$ $8.63(d, J=3.6 \mathrm{~Hz}, 1 \mathrm{H}), 8.36(s, 1 \mathrm{H}, \mathrm{CH}=\mathrm{N}), 8.01(t, J=16.1 \mathrm{~Hz}, 1 \mathrm{H}), 7.73(t, J=7.3 \mathrm{~Hz}, 1 \mathrm{H}), 7.35-7.27$ $(m, 1 \mathrm{H}), 2.23-2.13\left(m, 3 \mathrm{H}, \mathrm{CH}\left(\mathrm{CH}_{2}\right)_{3}\right), 1.83\left(s, 6 \mathrm{H}, \mathrm{CH}\left(\mathrm{CH}_{2}\right)_{3}\right), 1.79-1.65\left(m, 6 \mathrm{H}, \mathrm{CH}\left(\mathrm{CH}_{2}\right)_{3}\right) .{ }^{13} \mathrm{C} \mathrm{NMR}$ $\left(126 \mathrm{MHz}_{2} \mathrm{CDCl}_{3}\right) \delta 156.08(\mathrm{CH}=\mathrm{N}), 155.58,149.17,136.38,124.24,120.82,58.02,42.89,36.44,29.44$. Anal. calcd. for $\mathrm{C}_{16} \mathrm{H}_{20} \mathrm{~N}_{2}$ : C, 79.96; $\mathrm{H}, 8.39 ; \mathrm{N}, 11.66$; found: $\mathrm{C}, 79.81 ; \mathrm{H}, 8.37 ; \mathrm{N}, 11.72$.

Triphenyl(pyridin-2-yl-methylene)amine (L4): the white solid precipitated from the solution and was separated by filtration. The white solid was washed with methanol $(3 \times 5 \mathrm{~mL})$. Yield: $8.18 \mathrm{~g}(78.3 \%)$. ${ }^{1} \mathrm{H}$ NMR $\left(500 \mathrm{MHz}, \mathrm{CDCl}_{3}\right) \delta 8.61(d, J=3.9 \mathrm{~Hz}, 1 \mathrm{H}), 8.38(d, J=7.9 \mathrm{~Hz}, 1 \mathrm{H}, \mathrm{CH}=\mathrm{N}), 7.99(s, 1 \mathrm{H}), 7.81$ $(t, J=7.5 \mathrm{~Hz}, 1 \mathrm{H}), 7.34(m, 16 \mathrm{H},) .{ }^{13} \mathrm{C} \mathrm{NMR}\left(126 \mathrm{MHz}, \mathrm{CDCl}_{3}\right) \delta 160.95(\mathrm{CH}=\mathrm{N}), 155.27,149.28,145.25$, 136.61, 129.76, 127.85, 126.93, 124.84, 121.32. Anal. calcd. for $\mathrm{C}_{25} \mathrm{H}_{20} \mathrm{~N}_{2}$ : C, 86.17; $\mathrm{H}, 5.79 ; \mathrm{N}, 8.04$; found: C, 86.32; H, 5.63; N, 7.98 .

\subsection{General Procedure for the Synthesis of Iron Complexes}

All complexes were prepared in a similar manner by the reaction of anhydrous $\mathrm{FeCl}_{2}$ with the corresponding ligands in dichloromethane. A typical synthetic procedure used for complexes 1a, 3a, 4a, and $7 \mathbf{a}$ is as follows. Ligand $(1.0 \mathrm{mmol})$ and $\mathrm{FeCl}_{2}(1.0 \mathrm{mmol})$ were stirred in $10 \mathrm{~mL}$ of dichloromethane overnight at room temperature. The precipitate was collected by filtration, washed with hexane $(10 \mathrm{~mL} \times 2)$ and dried under vacuum to obtain orange, purple, or burgundy solid.

(CyclohexylIminopyridine) $\mathrm{FeCl}_{2}$ (1a) (purple solid, $0.30 \mathrm{~g}, 95 \%$ ): MALDI-TOF-MS ( $\mathrm{m} / \mathrm{z}$ ): calcd. for $\mathrm{C}_{12} \mathrm{H}_{16} \mathrm{ClFeN}_{2}$ : 279.0351, found: $278.9959[\mathrm{M}-\mathrm{Cl}]^{+}$. Anal. calcd. for $\mathrm{C}_{12} \mathrm{H}_{16} \mathrm{Cl}_{2} \mathrm{FeN}_{2}$ : C, 45.75; $\mathrm{H}, 5.12 ; \mathrm{N}, 8.89$; found: $\mathrm{C}, 46.20 ; \mathrm{H}, 4.99 ; \mathrm{N}, 9.12$. IR/ $\mathrm{cm}^{-1}: 1563, v(\mathrm{C}=\mathrm{N})$.

(Adamantyl Iminopyridine) $\mathrm{FeCl}_{2}$ (3a) (orange solid, $0.35 \mathrm{~g}, 95 \%$ ): MALDI-TOF-MS $(\mathrm{m} / \mathrm{z}$ ): calcd. for $\mathrm{C}_{16} \mathrm{H}_{20} \mathrm{ClFeN}_{2}$ : 331.0664, found: $330.9991[\mathrm{M}-\mathrm{Cl}]^{+}$. Anal. calcd. for $\mathrm{C}_{16} \mathrm{H}_{20} \mathrm{Cl}_{2} \mathrm{FeN}_{2}$ : C, 52.35; $\mathrm{H}, 5.49$; N, 7.63; found: C, 52.55; H, 5.33; N, 7.29. IR/ $\mathrm{cm}^{-1}: 1588, v(\mathrm{C}=\mathrm{N})$.

(Triphenyl Iminopyridine) $\mathrm{FeCl}_{2}$ (4a) (light-orange solid, $0.46 \mathrm{~g}, 96 \%$ ): MALDI-TOF-MS ( $\mathrm{m} / \mathrm{z}$ ): calcd. for $\mathrm{C}_{25} \mathrm{H}_{20} \mathrm{ClFeN}_{2}$ : 439.0664, found: $439.0714[\mathrm{M}-\mathrm{Cl}]^{+}$. Anal. calcd. for $\mathrm{C}_{25} \mathrm{H}_{20} \mathrm{Cl}_{2} \mathrm{FeN}_{2}: \mathrm{C}, 63.19 ; \mathrm{H}, 4.24$; $\mathrm{N}, 5.90$; found: $\mathrm{C}, 62.88 ; \mathrm{H}, 4.18 ; \mathrm{N}, 5.67 . \mathrm{IR} / \mathrm{cm}^{-1}: 1588, v(\mathrm{C}=\mathrm{N})$.

(dibenzhydryl Iminopyridine) $\mathrm{FeCl}_{2}$ (7a) (burgundy solid, $0.61 \mathrm{~g}, 93 \%$ ): MALDI-TOF-MS ( $\mathrm{m} / \mathrm{z}$ ): calcd. for $\mathrm{C}_{39} \mathrm{H}_{32} \mathrm{ClFeN}_{2}$ : 619.1603, found: 619.0020 [M - Cl] $]^{+}$. Anal. calcd. for $\mathrm{C}_{39} \mathrm{H}_{32} \mathrm{Cl}_{2} \mathrm{FeN}_{2}: \mathrm{C}, 71.47 ; \mathrm{H}, 4.92$; $\mathrm{N}, 4.27$; found: $\mathrm{C}, 71.99 ; \mathrm{H}, 4.87 ; \mathrm{N}, 4.17$. IR/ $\mathrm{cm}^{-1}: 1593, v(\mathrm{C}=\mathrm{N})$.

\subsection{General Procedure for the Synthesis of Cobalt Complexes}

All complexes were prepared in a similar manner by the reaction of anhydrous $\mathrm{CoCl}_{2}$ with the corresponding ligands in tetrahydrofuran (THF). A typical synthetic procedure used for complexes 
$\mathbf{2} \mathbf{b}, \mathbf{3} \mathbf{b}$, and $\mathbf{5 b}$ is as follows. Ligand $(1.0 \mathrm{mmol})$ and $\mathrm{CoCl}_{2}(1.0 \mathrm{mmol})$ were stirred in $10 \mathrm{~mL}$ of THF overnight at room temperature. The precipitate was collected by filtration, washed with hexane $(10 \mathrm{~mL} \times 2)$ and dried under vacuum to obtain a blue or green solid.

(octyl Iminopyridine) $\mathrm{CoCl}_{2}$ (2b) (blue solid, $0.30 \mathrm{~g}, 87 \%$ ): MALDI-TOF-MS $(\mathrm{m} / \mathrm{z}$ ): calcd. for $\mathrm{C}_{4} \mathrm{H}_{22} \mathrm{ClCoN}_{2}$ : 312.0804, found: $311.9917[\mathrm{M}-\mathrm{Cl}]^{+}$. Anal. calcd. for $\mathrm{C}_{4} \mathrm{H}_{22} \mathrm{Cl}_{2} \mathrm{CoN}_{2}: \mathrm{C}, 48.30 ; \mathrm{H}$, 6.37 ; N, 8.05; found: $\mathrm{C}, 49.41 ; \mathrm{H}, 6.45 ; \mathrm{N}, 7.91 . \mathrm{IR} / \mathrm{cm}^{-1}: 1597, v(\mathrm{C}=\mathrm{N})$.

(Adamantyl Iminopyridine) $\mathrm{CoCl}_{2}$ (3b) (blue solid, $0.33 \mathrm{~g}, 90 \%$ ): MALDI-TOF-MS $(\mathrm{m} / \mathrm{z}$ ): calcd. for $\mathrm{C}_{16} \mathrm{H}_{20} \mathrm{ClCoN}_{2}$ : 334.0647, found: $333.9984\left[\mathrm{M}-\mathrm{Cl}^{+}\right.$. Anal. calcd. for $\mathrm{C}_{16} \mathrm{H}_{20} \mathrm{Cl}_{2} \mathrm{CoN}_{2}: \mathrm{C}, 51.91$; H, 5.45; N, 7.57; found: C, 52.03; H, 5.23; N, 7.88. IR/ $\mathrm{cm}^{-1}: 1595, v(\mathrm{C}=\mathrm{N})$.

(supermesityl Iminopyridine) $\mathrm{CoCl}_{2}$ (5b) (green solid, $0.49 \mathrm{~g}$, 91\%): MALDI-TOF-MS ( $\mathrm{m} / \mathrm{z}$ ): calcd. for $\mathrm{C}_{30} \mathrm{H}_{22} \mathrm{ClCoN}_{2}$ : 504.0804, found: $503.9194\left[\mathrm{M}-\mathrm{Cl}^{+}\right.$. Anal. calcd. for $\mathrm{C}_{30} \mathrm{H}_{22} \mathrm{Cl}_{2} \mathrm{CoN}_{2}: \mathrm{C}, 66.68 ; \mathrm{H}$, 4.10; N, 5.18; found: $\mathrm{C}, 66.11 ; \mathrm{H}, 3.96 ; \mathrm{N}, 5.31$. IR/ $\mathrm{cm}^{-1}: 1597, v(\mathrm{C}=\mathrm{N})$.

\subsection{General Procedure for Isoprene Polymerization}

The polymerization of isoprene in toluene was carried out in a $50 \mathrm{~mL}$ Schlenk reactor. In a typical experiment, the reactor was heated, dried in a vacuum, and recharged with nitrogen more than three times before the required amount of an aluminum coactivator, toluene $(7 \mathrm{~mL})$, and isoprene $(2 \mathrm{~mL})$ were added into the reactor. Then, $8.0 \mu \mathrm{mol}$ of iron or cobalt complex in $1 \mathrm{~mL} \mathrm{CH} \mathrm{Cl}_{2}$ was injected to initiate the polymerization at the desired temperature. After $2 \mathrm{~h}$, the polymerization was quenched with a diluted $\mathrm{HCl}$ solution of methanol (methanol/ $\mathrm{HCl}=50 / 1$ ). The polymer was collected by filtration and washed with ethanol several times and dried at room temperature for $24 \mathrm{~h}$ under vacuum.

\subsection{Calculation of Microstructure Contents of Polyisoprenes}

According to the calculated area of the characteristic signals at $4.66-4.72$ and $5.12 \mathrm{ppm}$, the molar content of 3,4 units and 1,4 units based on ${ }^{1} \mathrm{H}$ NMR spectra can be calculated by Equations (1) and (2) where $I(5.12 \mathrm{ppm})$ and $I(4.66-4.72 \mathrm{ppm})$ represent signal areas at 5.12 and $4.66-4.72 \mathrm{ppm}$.

$$
\begin{aligned}
{[\% 1,4-\text { units }] } & =\frac{I(5.12 \mathrm{ppm})}{I(5.12 \mathrm{ppm})+\frac{I(4.66-4.72 \mathrm{ppm})}{2}} \\
{[\% 3,4-\text { units }] } & =\frac{\frac{I(4.66-4.72 \mathrm{ppm})}{2}}{I(5.12 \mathrm{ppm})+\frac{I(4.66-4.72 \mathrm{ppm})}{2}}
\end{aligned}
$$

According to the calculated area of the characteristic signals at 16.2 and $23.8 \mathrm{ppm}$, the molar content of cis-1,4 units and trans-1,4 units based on ${ }^{13} \mathrm{C}$ NMR spectra can be calculated by Equations (3) and $(4)$, where $I(23.8 \mathrm{ppm})$ and $I(16.2 \mathrm{ppm})$ represent signal areas at 23.8 and $16.2 \mathrm{ppm}$.

$$
\begin{aligned}
{[\% \text { cis- } 1,4-\text { units }] } & =\frac{I(23.8 \mathrm{ppm})}{I(23.8 \mathrm{ppm})+I(16.2 \mathrm{ppm})} \\
{[\% \text { trans-1, } 4 \text {-units }] } & =\frac{I(16.2 \mathrm{ppm})}{I(23.8 \mathrm{ppm})+I(16.2 \mathrm{ppm})}
\end{aligned}
$$

The microstructures of the polyisoprenes based on the FTIR spectra can be calculated according to the equations in the literature [50].

$$
\begin{gathered}
A_{1375}=24[\text { cis-1, } 4 \text {-units }] L+32.6[3,4 \text {-units }] L \\
A_{890}=101[3,4 \text {-units }] L \\
{[\% \text { cis-1,4-units }]=100 \times \frac{[\text { cis-1, } 4 \text {-units }]}{[\text { cis-1, } 4 \text {-units }]+[3,4 \text {-units }]}}
\end{gathered}
$$




$$
[\% 3,4 \text {-units }]=100 \times \frac{[3,4 \text {-units }]}{[\text { cis-1,4-units }]+[3,4 \text {-units }]}
$$

where $A_{1375}$ and $A_{890}$ are the absorption intensity at 1375 and $890 \mathrm{~cm}^{-1}$, expressed by the peak height, [cis-1,4-units] represents the molar content of cis-1,4-units, [3,4-units] represents the molar content of 3,4-units, and $L$ indicates the thickness of the sample.

\section{Results and Discussion}

\subsection{Synthesis and Characterization of the Iron and Cobalt Complexes}

The synthetic route for the iminopyridine complexes is shown in Scheme 2. The ligands were prepared at high yields by acid-catalyzed condensation between corresponding anilines and 2-pyridinecarboxaldehyde in methanol and identified by NMR (See Supplementary Materials, Figures S1-S6) and elemental analysis. The corresponding Fe(II) and Co(II) complexes (1a-7a, 2b, $3 \mathbf{b}, 5 \mathbf{b}, 6 \mathbf{b}$ ) were prepared from the reaction of the ligands with 1 equiv of anhydrous $\mathrm{FeCl}_{2}$ or $\mathrm{CoCl}_{2}$ in $\mathrm{CH}_{2} \mathrm{Cl}_{2}$ and THF, respectively. These complexes were characterized by mass spectroscopy (See Supplementary Materials, Figures S7-S13) and elemental analysis.

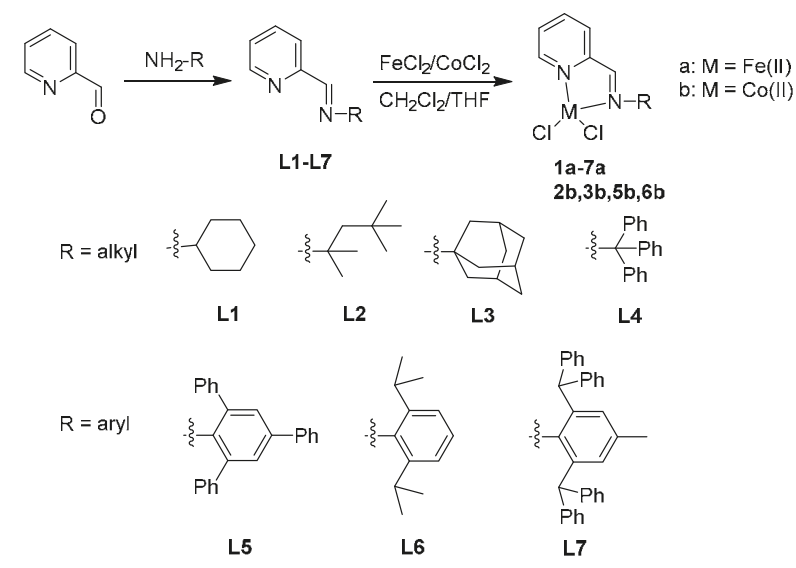

Scheme 2. Synthesis of the ligands and the Fe(II) and Co(II) complexes.

The structures of the complexes 1a-7a should be those drawn in Scheme 2. This is supported by the elemental analysis, mass spectroscopy, and literature results on similar Fe(II) complexes [51]. Multiple attempts to grow single crystals of complexes 1a-7a failed. However, during this process, single crystals of complex $7 \mathbf{a}^{\prime}$ were obtained and analyzed by X-ray diffraction (Figure 1, See Supplementary Materials, Tables S2 and S3). Complex $7 \mathbf{a}^{\prime}$ probably arises from the oxidation of $7 \mathbf{a}$ during the recrystallization process. This unusual complex of $7 \mathbf{a}^{\prime}$ is interesting, and can prove the connectivity of the iminopyridine ligand to the metal center. The X-ray crystal structure analysis of $7 \mathbf{a}^{\prime}$ shows a distorted trigonal bipyramidal coordination geometry around the $\mathrm{Fe}(\mathrm{II})$ center. The steric environment of the ligand and the blocking of the axial position of the metal center from the dibenzhydryl moiety can be clearly observed from this molecular structure. Single crystals of pure complex $\mathbf{2 b}$ could be obtained and the X-ray structure is shown in Figure 2. In a solid state, the cobalt center adopts a distorted tetrahedral coordination geometry with $\mathrm{N} 1-\mathrm{Co}-\mathrm{N} 2$ angle of $81.61^{\circ}$ and $\mathrm{Cl} 1-\mathrm{Co}-\mathrm{Cl} 2$ angle of $112.06^{\circ}$ (See Supplementary Materials, Tables S2 and S4). Complex $\mathbf{2 b}$ shows shorter $\mathrm{Co}-\mathrm{N}$ bond distance (2.040 and 2.046 $⿱$ ) than aryl-substituted Co(II) complexes reported in literature [41] (2.044 2.181 $)$ ), which may be attributed to the strong electron-donating effect of the octyl substituents. 


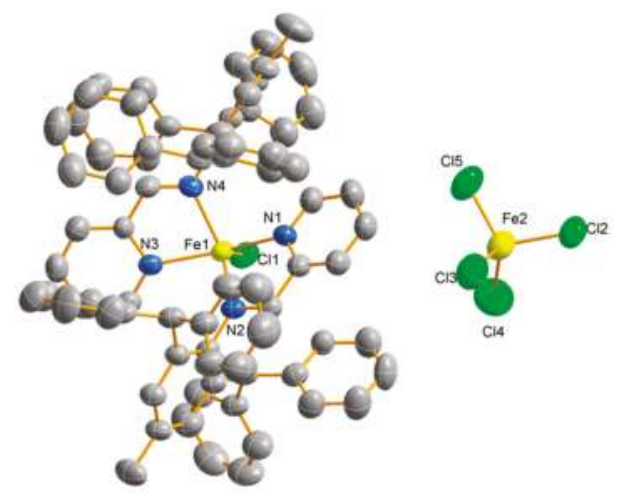

Figure 1. Molecular structure of complex $7 \mathbf{a}^{\prime}$. (Thermal ellipsoids are shown at the $50 \%$ probability level.) Hydrogen atoms have been omitted for clarity.

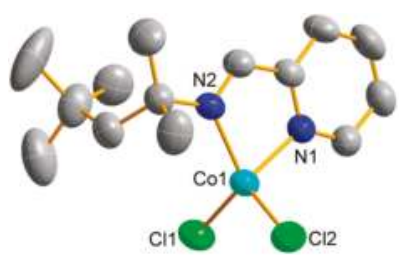

Figure 2. Molecular structure of complex 2b. (Thermal ellipsoids are shown at the $50 \%$ probability level.) Hydrogen atoms have been omitted for clarity.

\subsection{Isoprene Polymerization Studies}

\subsubsection{Polymerization of Isoprene with Iron Catalysts}

The isoprene polymerization was evaluated using various common alkylaluminum reagents as cocatalysts. Triisobutylaluminum (TIBA) or $\mathrm{AlEt}_{2} \mathrm{Cl}$ cocatalysts were not effective at all. Both $\mathrm{AlEtCl}_{2}$ and MAO were able to activate 2a for isoprene polymerization (Table 1, entries 1 and 2). However, the 2a/MAO system can generate high molecular polyisoprenes. Therefore, MAO was chosen as the activator in the iminopyridine Fe(II) system (See Supplementary Materials, Table S1).

Table 1. Isoprene polymerization results with Fe(II) catalyst ${ }^{\mathrm{a}}$.

\begin{tabular}{|c|c|c|c|c|c|c|c|c|c|c|}
\hline \multirow{2}{*}{ Entry } & \multirow{2}{*}{ Complex } & \multirow{2}{*}{$\begin{array}{c}T \\
\left({ }^{\circ} \mathrm{C}\right)\end{array}$} & \multirow{2}{*}{$\begin{array}{c}\text { Yield } \\
(\%)\end{array}$} & \multirow{2}{*}{ Activity $^{c}$} & \multirow{2}{*}{$\underset{\left(\times 10^{-4}\right)}{M_{\mathrm{n}}^{\mathrm{d}}}$} & \multirow{2}{*}{ PDI $^{d}$} & \multicolumn{4}{|c|}{ Microstructure $^{c}(\%){ }^{e}$} \\
\hline & & & & & & & cis $-1,4$ & trans $-1,4$ & cis/trans & 3,4 \\
\hline $1^{b}$ & $2 a$ & 25 & 83.4 & 7.1 & 0.18 & 4.70 & - & - & & - \\
\hline 2 & $2 a$ & 25 & 83.1 & 7.1 & 6.1 & 1.57 & 77.5 & 8.1 & $91: 9$ & 14.4 \\
\hline 3 & $2 a$ & -25 & 66.3 & 5.6 & 7.9 & 2.45 & 77.0 & 8.7 & $90: 10$ & 14.3 \\
\hline 4 & $1 a$ & 25 & 64.1 & 5.4 & 6.0 & 2.11 & 77.1 & 8.9 & $90: 10$ & 14.0 \\
\hline 5 & $3 a$ & 25 & 58.2 & 4.9 & 7.0 & 1.82 & 76.8 & 8.2 & $90: 10$ & 15.0 \\
\hline 6 & $4 a$ & 25 & 61.3 & 5.2 & 6.1 & 2.08 & 78.2 & 7.6 & $91: 9$ & 14.2 \\
\hline 7 & $5 a$ & -25 & 81.0 & 6.9 & 15.4 & 2.13 & 63.9 & 3.0 & $96: 4$ & 33.1 \\
\hline 8 & $5 a$ & 25 & 98.1 & 8.3 & 10.3 & 2.05 & 62.7 & 2.8 & $96: 4$ & 34.5 \\
\hline 9 & $6 a$ & 25 & 83.2 & 7.1 & 18.0 & 1.75 & 69.9 & 4.5 & $94: 6$ & 25.6 \\
\hline 10 & $7 a$ & 25 & 85.7 & 7.3 & 18.2 & 1.61 & 71.4 & 4.8 & $94: 6$ & 23.8 \\
\hline
\end{tabular}

a Polymerization conditions: $8.0 \mu \mathrm{mol}$ of $\mathrm{Fe}(\mathrm{II})$ complex; $\mathrm{MAO} / \mathrm{Fe}=500 ; 7 \mathrm{~mL}$ toluene and $1 \mathrm{~mL} \mathrm{CH}_{2} \mathrm{Cl}_{2}$; isoprene $=2 \mathrm{~mL}$; time $=2 \mathrm{~h} ;{ }^{\mathrm{b}}$ activator $=\mathrm{AlEtCl}_{2}, \mathrm{Al} / \mathrm{Fe}=150 ;{ }^{\mathrm{c}} 10^{4} \mathrm{~g}$ of polyisorene $(\mathrm{mol} \mathrm{of} \mathrm{Fe})^{-1} \cdot \mathrm{h}^{-1}$; ${ }^{d}$ determined by gel permeation chromatography (GPC); ${ }^{e}$ determined by ${ }^{1} \mathrm{H}$ NMR and ${ }^{13} \mathrm{C}$ NMR. 
The alkyl and aryl moiety significantly influenced the catalytic performances of the complexes. The aryl-substituted complexes 5a-7a produced polymers at higher yields $(83.2 \%-98.1 \%)$ than the alkyl-substituted complexes 1a-4a $(58.2 \%-83.1 \%)$. The aryl moiety is electronically more withdrawing than the alkyl moiety, which can reduce the electron density on the metal center, leading to better monomer coordination and faster chain propagation. This is supported by the fact that complex $\mathbf{5 a}$ bears the strongest electron-withdrawing substituent and displays the highest yield. In addition, the molecular weight of polyisoprenes obtained by aryl-substituted complexes $\mathbf{5 a}-\mathbf{7 a}$ is higher than alkyl-substituted complexes 1a-4a $\left(10.3 \times 10^{4} \sim 18.2 \times 10^{4}\right.$ vs. $\left.6.0 \times 10^{4} \sim 7.9 \times 10^{4}\right)$. Probably, the steric environment of the aryl moiety retards chain transfer reaction more effectively than the alkyl moiety (See Supplementary Materials, Figures S14-S20). This is supported by the fact that complex 7a bears a sterically bulky dibenzhydryl-derived ligand framework and generates polyisoprene with the highest molecular weight $\left(18.2 \times 10^{4}\right)$. The temperature influence on the catalytic performance was also investigated. Polymerization of isoprene at $-25^{\circ} \mathrm{C}$ showed lower yields (2a: $66.3 \%$ vs. $83.1 \%$; $5 \mathrm{a}$ : $81.0 \%$ vs. $98.1 \%)$ and afforded the polymer with higher molecular weight $\left(2 \mathrm{a}: 7.9 \times 10^{4}\right.$ vs. $6.1 \times 10^{4}$; 5a: $15.4 \times 10^{4}$ vs. $10.3 \times 10^{4}$ ) than those at $25^{\circ} \mathrm{C}$.

The microstructures of the resulting polyisoprenes were analyzed via ${ }^{1} \mathrm{H}$ NMR and ${ }^{13} \mathrm{C} N M R$ (See Supplementary Materials, Figures S23-S26) [51]. The representative ${ }^{1} \mathrm{H}$ NMR spectra of the polyisoprenes obtained by the Fe(II) catalysts are shown in Figure 3. The 1,2-unit was not observed. The polyisoprene obtained by aryl-substituted complex 5 a contains $34.5 \%$ 3,4-units (Table 1, entry 10), which was much higher than that of the aryl-substituted complex 3a (15.0\%, entry 5). Similar trends were observed for other alkyl-substituted complexes (14.0\% 15.0\%) and aryl-substituted complexes (greater than $23.8 \%$ ). Interestingly, the $\mathrm{R}$ group in the alkyl-substituted complexes only slightly influenced 3,4-selectivity from $14.0 \%$ to $15.0 \%$. However, the selectivity of 3,4-units was increased from $23.8 \%$ to $34.5 \%$ when the steric hindrance of the aryl-substituted complexes was decreased. The high 3,4 units of polyisoprene can increase the toughness of the synthetic rubber and show outstanding wet skid resistance and low heat build-up when applied as car tires [55], thus representing a big advantage of this catalyst system. Additionally, the alkyl-substituted complexes 1a-4a produced polymers with higher cis-1,4 content $(77.1 \% \sim 78.2 \%)$ than the aryl-substituted complexes $5 \mathbf{a}-7 \mathbf{a}(62.7 \%-71.4 \%)$. At the same time, the alkyl-substituted complexes produced polyisoprene with $7.6 \% \sim 8.9 \%$ trans- 1,4 content, which was ca. twice as much as that by aryl-substituted complexes $(2.8 \% \sim 4.8 \%)$. However, polymers generated from the aryl-substituted complexes had the higher cis-1,4/trans-1,4 ratio (e.g., 5a: 96:4, entry 8) than the alkyl-substituted complexes (e.g., 3a: 90:10, entry 5). These results indicated that the electron-donating alkyl-substituted complexes tend to polymerize isoprene with trans-1,4-selectivity when 1,4-addition occurred. It was also observed that the steric hindrance of both kinds of complexes almost have minimum influence on cis-1,4/trans-1,4 stereoselectivity with $\mathbf{1 a}-\mathbf{4 a}$ (ca. 90:10) and 5a-7a (ca. 95:5).

Previously, Raynaud et al. used alkylaluminum $/\left[\mathrm{Ph}_{3} \mathrm{C}\right]\left[\mathrm{B}\left(\mathrm{C}_{6} \mathrm{~F}_{5}\right)_{4}\right]$ cocatalysts to activate the $\mathrm{Fe}(\mathrm{II})$ complexes, and the 1,4-trans/1,4-cis selectivity was affected by the alkyl/aryl substituents and the

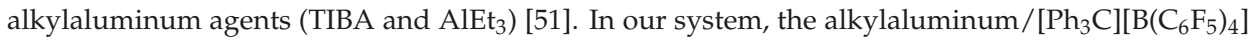
cocatalysts led to highly unreproducible results, which may originate from the high sensitivity of these $\mathrm{Fe}(\mathrm{II})$ complexes. As a result, MAO was chosen as the cocatalyst. These Fe(II) complexes showed high activities and high polymer molecular weight when activated using MAO as cocatalyst. Furthermore, in our $\mathrm{Fe}(\mathrm{II})$ /MAO system, the aryl-substituted iminopyridine iron complexes also favor 3,4-insertion and give rise to higher amounts of 3,4-units than the alkyl-substituted iminopyridine iron complexes, which is similar to the $\mathrm{Fe}(\mathrm{II}) /$ alkylaluminum/ $\left./ \mathrm{Ph}_{3} \mathrm{C}\right]\left[\mathrm{B}\left(\mathrm{C}_{6} \mathrm{~F}_{5}\right)_{4}\right]$ system. However, there are some notable differences between these two systems. In our $\mathrm{Fe}(\mathrm{II}) / \mathrm{MAO}$ system, the ratio between 1,4-cis/trans units was not affected by the aryl or alkyl substituents. Although this difference is not fully understood, it is clear that the cocatalysts may play an important role in determining the stereoselectivity. 


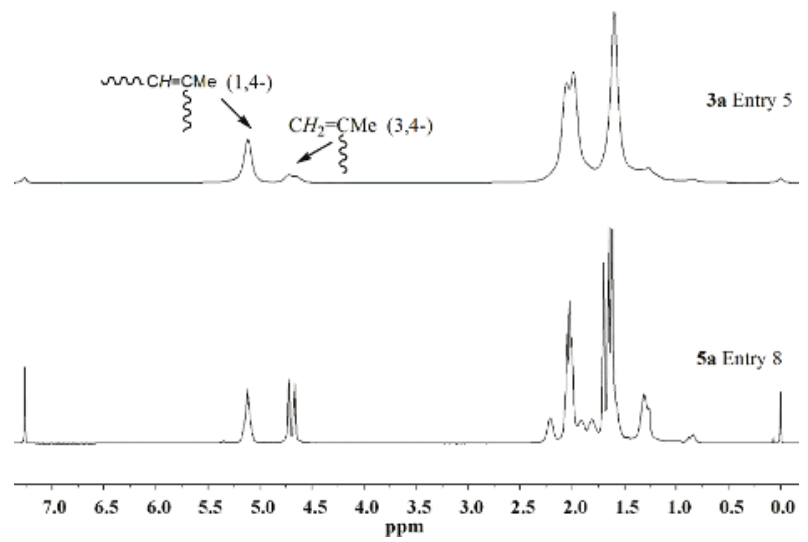

Figure 3. ${ }^{1} \mathrm{H}$ NMR spectra of polyisoprenes obtained by Fe(II) catalyst.

\subsubsection{Polymerization of Isoprene with Co(II) Catalysts}

The polymerization results using $\mathrm{Co}(\mathrm{II})$ complexes $\mathbf{2} \mathbf{b}, \mathbf{3} \mathbf{b}, \mathbf{5} \mathbf{b}$, and $\mathbf{6} \mathbf{b}$ are summarized in Table 2 . Four cocatalysts ( $\mathrm{TIBA}, \mathrm{AlEt}_{2} \mathrm{Cl}, \mathrm{AlEtCl}_{2}$, and $\mathrm{MAO}$ ) were used in attempts to generate the active catalysts. Only cocatalyst $\mathrm{AlEtCl}_{2}$ was able to activate $\mathrm{Co}(\mathrm{II})$ complex $\mathbf{2 b}$ for isoprene polymerization. Although the yields (greater than $76.9 \%$ ) of polyisoprene generated from $\mathrm{Co}$ (II) complexes are similar with those of $\mathrm{Fe}(\mathrm{II})$ complexes, there are some apparent differences between the $\mathrm{Co}(\mathrm{II})$ and the $\mathrm{Fe}$ (II) systems. In sharp contrast to the Fe(II) complexes, the polymers produced by $\mathrm{Co}(\mathrm{II})$ complexes were white powder with molecular weights below 2000 and broad molecular distribution of above 4.76 (See Supplementary Materials, Figures S21 and S22). Moreover, complexes $\mathbf{5 b}$ and $\mathbf{6 b}$ containing electron-withdrawing aryl substituents afforded polymers with higher molecular weights $(5 \mathbf{b}$ : 1700 , 6b: 1800 ) at higher yields ( $5 \mathbf{b}: 97.3 \%, \mathbf{6} \mathbf{b}: \mathbf{9 4 . 9} \%$ ) than those by complexes $\mathbf{2} \mathbf{b}$ and $\mathbf{3 b}$ containing electron-donating alkyl substituents (2b: 1400 and $78.2 \%, 3 \mathbf{b}$ : 1500 and $76.9 \%)$. This is similar with the trend observed in the $\mathrm{Fe}(\mathrm{II})$ systems.

Table 2. Isoprene polymerization results with Co(II) catalyst ${ }^{\mathrm{a}}$.

\begin{tabular}{cccccccc}
\hline \multirow{2}{*}{ Entry } & Complex & Yield (\%) & Activity $^{\mathbf{b}}$ & $\boldsymbol{M}_{\mathbf{n}}{ }^{\mathbf{c}}\left(\times \mathbf{1 0}^{-\mathbf{3}}\right)$ & PDI $^{\mathbf{c}}$ & \multicolumn{2}{c}{ Microstructure $^{\mathbf{d}} \mathbf{( \% )}$} \\
\hline 1 & $\mathbf{2 b}$ & 78.2 & 6.6 & 1.4 & 7.97 & 91.1 & 8.9 \\
2 & $\mathbf{3 b}$ & 76.9 & 6.5 & 1.5 & 4.76 & 90.8 & 9.2 \\
3 & $\mathbf{5 b}$ & 97.3 & 8.3 & 1.7 & 8.05 & 88.1 & 11.9 \\
4 & $\mathbf{6 b}$ & 94.9 & 8.1 & 1.8 & 9.38 & 89.7 & 10.3 \\
\hline
\end{tabular}

${ }^{a}$ Polymerization conditions: $8.0 \mu \mathrm{mol}$ of $\mathrm{Co}(\mathrm{II})$ complex; activator $=\mathrm{AlEtCl}_{2}, \mathrm{Al} / \mathrm{Co}=150 ; 7 \mathrm{~mL}$ toluene and $1 \mathrm{~mL} \mathrm{CH} \mathrm{Cl}_{2}$; isoprene $=2 \mathrm{~mL}$; time $=2 \mathrm{~h} ;{ }^{\mathrm{b}} 10^{4} \mathrm{~g}$ of polyisorene $(\mathrm{mol} \mathrm{of} \mathrm{Co})^{-1} \cdot \mathrm{h}^{-1} ;^{\mathrm{c}}$ determined by GPC;

d determined by Fourier-transform infrared (FTIR) spectroscopy.

The ${ }^{1} \mathrm{H}$ NMR and ${ }^{13} \mathrm{C}$ NMR spectra of polyisoprene obtained by Co(II) complexes have the broad peaks and low resolution because of the low molecular weight of the polymers (See Supplementary Materials, Figures S27-S30). It was difficult to assign the peaks of these polymers in the ${ }^{1} \mathrm{H}$ NMR and ${ }^{13} \mathrm{C}$ NMR spectra, so FTIR measurements were carried out to determine and analyze the microstructures of the polyisoprenes (See Supplementary Materials, Figures S31-S34). The absorption bands at 1375 and $890 \mathrm{~cm}^{-1}$ correspond to the cis-1,4 and the 3,4-units [50]. The typical bands of trans-1,4 units are at $845,1152,1325$, and $1385 \mathrm{~cm}^{-1}$ and the band of 1,2-units is at $911 \mathrm{~cm}^{-1}$ [50]. As shown in Figure 4, no bands were observed for the trans-1,4 unit or 1,2-unit in the spectrum. Based on the 
equations shown in the experimental section, the polymer generated with the $\mathrm{Co}(\mathrm{II}) / \mathrm{AlEtCl}_{2}$ system is composed of predominantly cis-1,4 units (ca. 90\%) along with a small amount of 3,4-units (ca. 10\%). Interestingly, the $\mathrm{Co}$ (II) system produced polymers with higher cis-1,4 content (ca. 90\%) than the $\mathrm{Fe}$ (II) system $(65 \% \sim 85 \%)$. The stereoregularity of the polyisoprenes was only slightly influenced by the ligand environment.

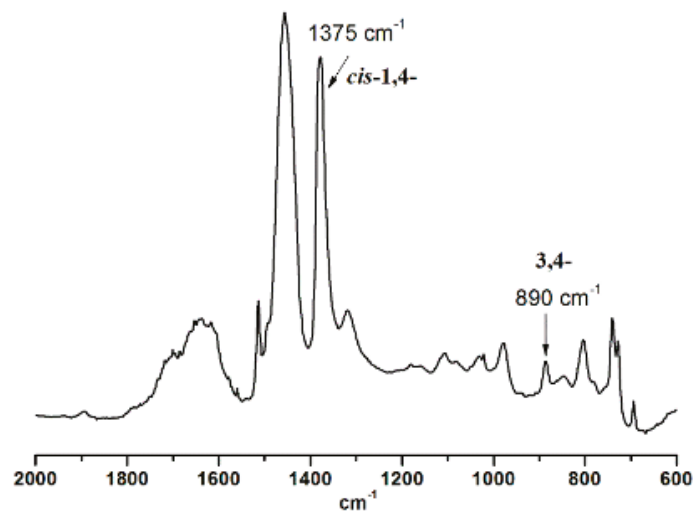

Figure 4. FTIR spectra of polyisoprenes obtained by Co(II) catalysts (entry 6).

\section{Conclusions}

In conclusion, a series of iminopyridine Fe(II) and Co(II) complexes bearing various alkyl and aryl substituents was prepared. The aim is to systematically investigate the influence of alkyl and aryl substituents on the isoprene polymerization. Activated by MAO, the Fe(II) complexes exhibited moderate cis-1,4 selectivity, generating high molecular weight polyisoprenes. The Fe(II) catalyzed polymerization of isoprene was relatively sensitive to alkyl and aryl substituents. High 3,4-units (up to $34.5 \%$ ) and high molecular weight $\left(10.3 \times 10^{4} \sim 18.2 \times 10^{4}\right)$ polyisoprenes can be obtained using aryl-substituted $\mathrm{Fe}(\mathrm{II})$ complexes. Meanwhile, the $\mathrm{Co}(\mathrm{II}) / \mathrm{AlEt}_{2} \mathrm{Cl}$ system exhibited relatively high cis-1,4-selectivity, affording low molecular weight polyisoprenes. The alkyl and aryl substituents in $\mathrm{Co}(\mathrm{II})$ complexes did not significantly influence the selectivity and molecular weight of the resulting polymers.

Supplementary Materials: The following are available online at www.mdpi.com/2073-4360/8/11/389/s1 Optimization of MAO/Fe Ratio with 3a (Table S1), NMR spectra of the ligands L1, L3 and L4 (Figures S1-S6), MALDI-TOF-MS of complexes (Figures S7-S13), crystal data of complex 7a' (CCDC number: 1503575) and 2b (CCDC number: 1503576) (Tables S2-S4), GPC curves of polyisoprene samples (Figures S14-S22), NMR spectra of the representive polyisoprenes (Figures S23-S30) and FTIR spectra of representive polyisoprenes (Figures S31-S34).

Acknowledgments: This work was supported by National Natural Science Foundation of China (NSFC, 21304054, 21374108 and 51522306), Foundation of Qufu Normal University (xkJ201603), National College Students Innovation Project (201610446029), Anhui Provincial Natural Science Foundation (1408085QB28, 1608085MB29) and the Recruitment Program of Global Experts.

Author Contributions: Lihua Guo and Changle Chen conceived and designed the experiments; Lihua Guo, Xinyu Jing, Shuoyan Xiong, Wenjing Liu, Yanlan Liu and Zhe Liu performed the experiments; Lihua Guo and Changle Chen analyzed the data and wrote the paper.

Conflicts of Interest: The authors declare no conflict of interest.

\section{References}

1. Horne, S.E., Jr.; Kiehl, J.P.; Shipman, J.J.; Folt, V.L.; Gibbs, C.F. Ameripol SN-A cis-1,4-polyisoprene. Ind. Eng. Chem. 1956, 48, 784-791. [CrossRef]

2. Natta, G. Progress in five years of research in stereospecific polymerization. SPE J. 1959, 53, 373-382. 
3. Proto, A.; Capacchione, C. Stereoselective Polymerization with Single-Site Catalysts; Baugh, L.S., Canich, J.A.M., Eds.; CRC Press: Boca Raton, FL, USA, 2008.

4. Ricci, G.; Sommazzi, A.; Masi, F.; Ricci, M.; Boglia, A.; Leone, G. Well-defined transition metal complexes with phosphorus and nitrogen ligands for 1,3-dienes polymerization. Coord. Chem. Rev. 2010, 254, 661-676. [CrossRef]

5. Osakada, K.; Takeuchi, D. Coordination polymerization of dienes, allenes, and methylenecycloalkanes. Adv. Polym. Sci. 2004, 171, 137-194.

6. Zhang, L.X.; Suzuki, T.; Luo, Y.; Nishiura, M.; Hou, Z.M. Cationic alkyl rare-earth metal complexes bearing an ancillary Bis(phosphinophenyl)amido ligand: A catalytic system for living cis-1,4-polymerization and copolymerization of isoprene and butadiene. Angew. Chem. Int. Ed. 2007, 46, 1909-1913. [CrossRef] [PubMed]

7. Gao, W.; Cui, D.M. Highly cis-1,4 selective polymerization of dienes with homogeneous Ziegler-Natta catalysts based on NCN-pincer rare earth metal dichloride precursors. J. Am. Chem. Soc. 2008, 130, 4984-4991. [CrossRef] [PubMed]

8. Zhang, L.X.; Nishiura, M.; Yuki, M.; Luo, Y.; Hou, Z.M. Isoprene polymerization with yttrium amidinate catalysts: Switchingthe regio-and stereoselectivity by addition of $\mathrm{AlMe}_{3}$. Angew. Chem. Int. Ed. 2008, 47, 2642-2645. [CrossRef] [PubMed]

9. Li, D.F.; Li, S.H.; Cui, D.M.; Zhang, X.Q. $\beta$-diketiminato rare-earth metal complexes. Structures, catalysis, and active species for highly cis-1,4-selective polymerization of isoprene. Organometallics 2010, 29, 2186-2193. [CrossRef]

10. Lv, K.; Cui, D.M. CCC-pincer bis(carbene) lanthanide dibromides. Catalysis on highlycis-1,4-selective polymerization of isoprene and active species. Organometallics 2010, 29, 2987-2993. [CrossRef]

11. Nishiura, M.; Hou, Z.M. Novel polymerization catalysts and hydride clusters from rare-earth metal dialkyls. Nat. Chem. 2010, 2, 257-268. [CrossRef] [PubMed]

12. Milione, S.; Cuomo, C.; Capacchione, C.; Zannoni, C.; Grassi, A.; Proto, A. Stereoselective polymerization of conjugated dienes and styrene-butadiene copolymerization promoted by octahedral titanium catalyst. Macromolecules 2007, 40, 5638-5643. [CrossRef]

13. Buonerba, A.; Fienga, M.; Milione, S.; Cuomo, C.; Grassi, A.; Proto, A.; Capacchione, C. Binary copolymerization of $p$-methylstyrene with butadiene and isoprene catalyzed by titanium compounds showing different stereoselectivity. Macromolecules 2013, 46, 8449-8457. [CrossRef]

14. Proto, A.; Avagliano, A.; Saviello, D.; Ricciardi, R.; Capacchione, C. Living, isoselective polymerization of styrene and formation of stereoregular block copolymers via sequential monomer addition. Macromolecules 2010, 43, 5919-5921. [CrossRef]

15. Capacchione, C.; Saviello, D.; Ricciardi, R.; Proto, A. Living, isoselective polymerization of 4-methyl-1,3pentadiene and styrenic monomers and synthesis of highly stereoregular block copolymers via sequential monomer addition. Macromolecules 2011, 44, 7940-7947. [CrossRef]

16. Johnson, L.K.; Killian, C.M.; Brookhart, M. New Pd(II)- and Ni(II)-based catalysts for polymerization of ethylene and $\alpha$-olefins. J. Am. Chem. Soc. 1995, 117, 6414-6415. [CrossRef]

17. Nakamura, A.; Ito, S.; Nozaki, K. Coordination-insertion copolymerization of fundamental polar monomers. Chem. Rev. 2009, 109, 5215-5244. [CrossRef] [PubMed]

18. Chen, E.Y.X. Coordination polymerization of polar vinyl monomers by single-site metal catalysts. Chem. Rev. 2009, 109, 5157-5214. [CrossRef] [PubMed]

19. Guan, Z.; Cotts, P.M.; McCord, E.F.; McLain, S.J. Chain Walking: A new strategy to control polymer topology. Science 1999, 283, 2059-2061. [CrossRef] [PubMed]

20. Vaidya, T.; Klimovica, K.; LaPointe, A.M.; Keresztes, I.; Lobkovsky, E.B.; Daugulis, O.; Coates, G.W. Secondary alkene insertion and precision chain-walking: A new route to semicrystalline "polyethylene" from $\alpha$-olefins by combining two rare catalytic events. J. Am. Chem. Soc. 2014, 136, 7213-7216. [CrossRef] [PubMed]

21. Takano, S.; Takeuchi, D.; Osakada, K.; Akamatsu, N.; Shishido, A. Dipalladium catalyst for olefin polymerization: Introduction of acrylate units into the main chain of branched polyethylene. Angew. Chem. Int. Ed. 2014, 53, 9246-9250. [CrossRef] [PubMed]

22. Guo, L.H.; Chen, C.L. ( $\alpha$-Diimine) palladium catalyzed ethylene polymerization and copolymerization with polar comonomers. Sci. China Chem. 2015, 58, 1663-1673. [CrossRef]

23. Guo, L.H.; Dai, S.Y.; Sui, X.L.; Chen, C.L. Palladium and nickel catalyzed chain walking olefin polymerization and copolymerization. ACS Catal. 2016, 6, 428-441. [CrossRef] 
24. Guo, L.; Gao, H.; Guan, Q.; Hu, H.; Deng, J.; Liu, J.; Liu, F.; Wu, Q. Substituent effects of the backbone in $\alpha$-diimine palladium catalysts on homo- and copolymerization of ethylene with methyl acrylate. Organometallics 2012, 31, 6054-6062. [CrossRef]

25. Guo, L.H.; Sui, X.L.; Dai, S.Y.; Chen, C.L. Ligand electronic effects on $\alpha$-diimine nickel(II) catalyzed ethylene polymerization. Polymers 2016, 8, 37. [CrossRef]

26. Dai, S.Y.; Chen, C.L. Direct synthesis of functionalized high-molecular-weight polyethylene by copolymerization of ethylene with polar monomers. Angew. Chem. Int. Ed. 2016, 55, 13281-13285. [CrossRef] [PubMed]

27. Dai, S.Y.; Sui, X.L.; Chen, C.L. Synthesis of high molecular weight polyethylene using iminopyridyl nickel catalysts. Chem. Commun. 2016, 52, 9113-9116. [CrossRef] [PubMed]

28. Wang, R.K.; Zhao, M.H.; Chen, C.L. Influence of ligand second coordination sphere effects on the olefin (co)polymerization properties of $\alpha$-diimine Pd(II) catalysts. Polym. Chem. 2016, 7, 3933-3938. [CrossRef]

29. Hu, X.H.; Dai, S.Y.; Chen, C.L. Ethylene polymerization by salicylaldimine nickel(II) complexes containing dibenzhydryl moiety. Dalton Trans. 2016, 45, 1496-1503. [CrossRef] [PubMed]

30. Wang, R.K.; Sui, X.L.; Pang, W.M.; Chen, C.L. Ethylene polymerization by xanthene bridged dinuclear $\alpha$-diimine Ni(II) complexes. Chem CatChem 2016, 8, 434-440. [CrossRef]

31. Chen, M.; Yang, B.P.; Chen, C.L. Redox-controlled olefin (co)polymerization catalyzed by ferrocene bridged phosphine-sulfonate palladium complexes. Angew. Chem. Int. Ed. 2015, 54, 15520-15524. [CrossRef] [PubMed]

32. Dai, S.Y.; Sui, X.L.; Chen, C.L. Highly robust Pd(II) $\alpha$-diimine catalysts for slow-chain-walking polymerization of ethylene and copolymerization with methyl acrylate. Angew. Chem. Int. Ed. 2015, 54, 9948-9953. [CrossRef] [PubMed]

33. Sui, X.L.; Dai, S.Y.; Chen, C.L. Ethylene polymerization and copolymerization with polar monomers by cationic phosphine phosphonic amide palladium complexes. ACS Catal. 2015, 5, 5932-5937. [CrossRef]

34. Chen, M.; Zou, W.P.; Cai, Z.G.; Chen, C.L. Norbornene homopolymerization and copolymerization with ethylene by phosphine-sulfonate nickel catalysts. Polym. Chem. 2015, 6, 2669-2676. [CrossRef]

35. Ricci, G.; Battistella, M.; Porri, L. Chemoselectivity and stereospecificity of chromium(II) catalysts for 1,3-diene polymerization. Macromolecules 2001, 34, 5766-5769. [CrossRef]

36. Bazzini, C.; Giarrusso, A.; Porri, L. Diethylbis(2,2'-bipyridine)iron/MAO. A very active and stereospecific catalyst for 1,3-diene polymerization. Macromol. Rapid Commun. 2002, 23, 922-927. [CrossRef]

37. Ricci, G.; Morganti, D.; Sommazzi, A.; Santi, R.; Masi, F. Polymerization of 1,3-dienes with iron complexes based catalysts influence of the ligand on catalyst activity and stereospecificity. J. Mol. Catal. A Chem. 2003, 204-205, 287-293. [CrossRef]

38. Bazzini, C.; Giarrusso, A.; Porri, L.; Pirozzi, B.; Napolitano, R. Synthesis and characterization of syndiotactic 3,4-polyisoprene prepared with diethylbis(2,2'-bipyridine)iron-MAO. Polymer 2004, 45, 2871-2875. [CrossRef]

39. Ricci, G.; Forni, A.; Boglia, A.; Sonzogni, M. New chromium(II) bidentate phosphine complexes: Synthesis, characterization, and behavior in the polymerization of 1,3-butadiene. Organometallics 2004, 23, 3727-3732. [CrossRef]

40. Ricci, G.; Forni, A.; Boglia, A.; Motta, T. Synthesis, structure, and butadiene polymerization behavior of alkylphosphine cobalt(II) complexes. J. Mol. Catal. A Chem. 2005, 226, 235-241. [CrossRef]

41. Dai, Q.Q.; Jia, X.Y.; Yang, F.; Bai, C.X.; Hu, Y.M.; Zhang, X.Q. Iminopyridine-based cobalt(II) and nikel(II) complexes: Synthesis, characterization, and their catalytic behaviors for 1,3-butadiene polymerization. Polymers 2016, 8, 12. [CrossRef]

42. Gong, D.R.; Dong, W.M.; Hu, Y.M.; Bi, J.F.; Zhang, X.Q.; Jiang, L.S. Syndiotactically enriched 1,2-selective polymerization of 1,3-butadiene initiated by iron catalysts based on a new class of donors. Polymer 2009, 50, 5980-5986. [CrossRef]

43. Cámpora, J.; Tabla, L.O.; Palma, P.; Álvarez, E.; Lahoz, F.; Mereiter, K. Synthesis and catalytic activity of cationic allyl complexes of nickel stabilized by a single $n$-heterocyclic carbene ligand. Organometallcs 2006, 25, 3314-3316. [CrossRef]

44. Ricci, G.; Forni, A.; Boglia, A.; Motta, T.; Zannoni, G.; Canetti, M.; Bertini, F. Synthesis and X-ray structure of $\mathrm{CoCl}_{2}\left(\mathrm{P}^{i} \mathrm{PrPh}_{2}\right)_{2}$. A new highly active and stereospecific catalyst for 1,2 polymerization of conjugated dienes when used in association with MAO. Macromolecules 2005, 38, 1064-1070. [CrossRef] 
45. Ricci, G.; Boglia, A.; Motta, T. Synthesis of new $\mathrm{Cr}$ (II) complexes with bidentate phosphine ligands and their behavior in the polymerization of butadiene influence of the phosphine bite angle on catalyst activity and stereoselectivity. J. Mol. Catal. A Chem. 2007, 267, 102-107. [CrossRef]

46. Ricci, G.; Leone, G.; Boglia, A.; Boccia, A.C.; Zetta, L. cis-1,4-alt-3,4 polyisoprene: Synthesis and characterization. Macromolecules 2009, 42, 9263-9267. [CrossRef]

47. Ricci, G.; Leone, G.; Boglia, A.; Bertini, F.; Boccia, A.C.; Zetta, L. Synthesis and characterization of isotactic 1,2-poly(E-3-methyl-1,3-pentadiene). Some remarks about the influence of monomer structure on polymerization stereoselectivity. Macromolecules 2009, 42, 3048-3056. [CrossRef]

48. Ricci, G.; Motta, T.; Boglia, A.; Alberti, E.; Zetta, L.; Bertini, F.; Arosio, P.; Famulari, A.; Meille, S.V. Synthesis, characterization, and crystalline structure of syndiotactic 1,2-polypentadiene: The trans polymer. Macromolecules 2005, 38, 8345-8352. [CrossRef]

49. Ricci, G.; Boglia, A.; Motta, T.; Bertini, F.; Boccia, A.C.; Zetta, L.; Alberti, E.; Famulari, A.; Arosio, P.; Meille, S.V. Synthesis and structural characterization of syndiotactic trans-1,2 and cis-1,2 polyhexadienes. J. Polym. Sci. A Polym. Chem. 2007, 45, 5339-5353. [CrossRef]

50. He, A.H.; Wang, G.; Zhao, W.Z.; Jiang, X.B.; Yao, W.; Sun, W.H. High cis-1,4 polyisoprene or cis-1,4/3,4 binary polyisoprene synthesized using 2-(benzimidazolyl)-6-(1-(arylimino)ethyl)pyridine cobalt(II) dichlorides. Polym. Int. 2013, 62, 1758-1766. [CrossRef]

51. Raynaud, J.; Wu, J.Y.; Ritter, T. Iron-catalyzed polymerization of isoprene and other 1,3-dienes. Angew. Chem. Int. Ed. 2012, 51, 11805-11808. [CrossRef] [PubMed]

52. Wu, J.Y.; Stanzl, B.N.; Ritter, T. A Strategy for the synthesis of well-defined iron catalysts and application to regioselective diene hydrosilylation. J. Am. Chem. Soc. 2010, 132, 13214-13216. [CrossRef] [PubMed]

53. Sun, W.H.; Song, S.J.; Li, B.X.; Redshaw, C.; Hao, X.; Li, Y.S.; Wang, F.S. Ethylene polymerization by 2-iminopyridylnickel halide complexes: Synthesis, characterization and catalytic influence of the benzhydryl group. Dalton Trans. 2012, 41, 11999-12010. [CrossRef] [PubMed]

54. Gibson, V.C.; O'Reilly, R.K.; Wass, D.F.; White, A.J.P.; Williams, D.J. Iron complexes bearing iminopyridine and aminopyridine ligands as catalysts for atom transfer radical polymerization. Dalton Trans. 2003. [CrossRef]

55. Wang, B.L.; Cui, D.M.; Lv, K. Highly 3,4-selective living polymerization of isoprene with rare earth metal fluorenyl $N$-heterocyclic carbene precursors. Macromolecules 2008, 41, 1983-1988. [CrossRef]

(C) 2016 by the authors. Licensee MDPI, Basel, Switzerland. This article is an open access article distributed under the terms and conditions of the Creative Commons Attribution (CC BY) license (http:/ / creativecommons.org/licenses/by/4.0/). 
Article

\title{
Multiresponsive Behavior of Functional Poly( $p$-phenylene vinylene)s in Water
}

\author{
Kanykei Ryskulova ${ }^{1}$, Anupama Rao Gulur Srinivas ${ }^{2,3}$, Thomas Kerr-Phillips ${ }^{2,3}$, Hui Peng ${ }^{4,5}$, \\ David Barker ${ }^{2}$, Jadranka Travas-Sejdic ${ }^{2,3}$ and Richard Hoogenboom ${ }^{1, *}$ \\ 1 Supramolecular Chemistry Group, Department of Organic and Macromolecular Chemistry, \\ Faculty of Science, Ghent University, Krijgslaan 281 S4, Ghent B-9000, Belgium; kanikey@gmail.com \\ 2 Polymer Electronics Research Center, School of Chemical Sciences, The University of Auckland, \\ Private Bag 92019, Auckland, New Zealand; gsanu85@gmail.com (A.R.G.S.); \\ tker016@aucklanduni.ac.nz (T.K.-P.); d.barker@auckland.ac.nz (D.B.); \\ j.travas-sejdic@auckland.ac.nz (J.T.-S.) \\ 3 MacDiarmid Institute for Advanced Materials and Nanotechnology, Victoria University of Wellington, \\ P.O. Box 600, Wellington, New Zealand \\ 4 Key Laboratory of Polarized Materials and Devices, Ministry of Education, East China Normal University, \\ Shanghai 200062, China; h.peng@auckland.ac.nz \\ 5 Collaborative Innovation Center of Extreme Optics, Shanxi University, Taiyuan 030006, Shanxi, China \\ * Correspondence: Richard.Hoogenboom@ugent.be; Tel.: +32-926-449-98; Fax: +32-926-444-82
}

Academic Editors: Alexander Böker and Frank Wiesbrock

Received: 18 August 2016; Accepted: 10 October 2016; Published: 18 October 2016

\begin{abstract}
The multiresponsive behavior of functionalized water-soluble conjugated polymers (CPs) is presented with potential applications for sensors. In this study, we investigated the aqueous solubility behavior of water-soluble CPs with high photoluminescence and with a particular focus on their $\mathrm{pH}$ and temperature responsiveness. For this purpose, two poly(phenylene vinylene)s (PPVs) - namely 2,5-substituted PPVs bearing both carboxylic acid and methoxyoligoethylene glycol units-were investigated, with different amount of carboxylic acid units. Changes in the $\mathrm{pH}$ and temperature of polymer solutions led to a response in the fluorescence intensity in a $\mathrm{pH}$ range from 3 to 10 and for temperatures ranging from 10 to $85^{\circ} \mathrm{C}$. Additionally, it is demonstrated that the polymer with the largest number of carboxylic acid groups displays upper critical solution temperature (UCST)-like thermoresponsive behavior in the presence of a divalent ion like $\mathrm{Ca}^{2+}$. The sensing capability of these water-soluble PPVs could be utilized to design smart materials with multiresponsive behavior in biomedicine and soft materials.
\end{abstract}

Keywords: polymeric sensor; water-soluble conjugated polymer; fluorescent sensor; reversible calcium binding; thermoresponsive polymer; upper critical solution temperature

\section{Introduction}

Polymers that respond to external stimuli such as $\mathrm{pH}$, temperature, ionic strength, light, and concentration are extensively studied for a broad range of applications, including biomedical applications like drug delivery [1,2], cell imaging [3-5], as well as biological [6] and optical sensing [7-9]. Polymer-based systems with responsive properties can straightforwardly be designed thanks to recent advances in polymer synthesis as well as better understanding of physicochemical properties at the molecular level [10-12]. Such responsive functions arise from the chemical or topological structure of the polymer and lead to the design of stimuli-responsive smart materials with tailored functions. In contrast to their small molecular analogs, macromolecular or polymeric sensors exhibit numerous advantageous features, such as adjustable water solubility, higher detection sensitivity, straightforward integration into sensing devices, better stability, biocompatibility, and sharpened responses [13]. 
The important role of temperature and $\mathrm{pH}$ in many biological processes has led to increased interest in constructing smart materials that respond to these parameters. To develop polymeric sensors for temperature and/or $\mathrm{pH}$, normally two routes can be followed. Either a water-soluble polymer is modified with a temperature- or $\mathrm{pH}$-sensitive dye $[3,4,7,14]$, or a temperature- or $\mathrm{pH}$-responsive polymer is modified with a solvatochromic dye [15-18] to translate the polymer phase transition into a fluorescent or absorbance output signal. Whereas a variety of polymeric sensors are reported in literature either for $\mathrm{pH}$ [19-21] or temperature[22,23], only a few dual-responsive polymers for both $\mathrm{pH}$ and temperature exist $[3,7,24]$. A recent emerging strategy to design such dual-responsive system incorporates a $\mathrm{pH}$-responsive solvatochromic dye, such as Disperse Red 1, into a thermoresponsive polymer $[3,25]$.

Conjugated polymers (CPs) are widely applied for sensing applications. CPs provide a platform for prominent detection of chemical and biological entities due to a highly delocalized electronic backbone structure and optical signal amplification that are sensitive to changes in the structure induced by minor quantities of analytes [26-29]. For biomedical applications and aqueous sensing, water-soluble CPs are required, which can be achieved by introducing ionic or polar functional groups to the side chains, such as sulfonate, phosphate, carboxylate, quaternary ammonium, and ethylene glycol units $[8,13]$. Such water-soluble CPs have been utilized to develop biosensors for detection of DNA strands [30-34] and proteins [35,36] and for cellular imaging [3,37]. Aggregation induced by the analyte, which results in a change of the spectral signal (absorbance or fluorescence), is the most common method for analyte sensing with CPs [38,39], although aggregation can also lead to fluorescence quenching or signal enhancement [40].

Carboxylate-functionalized conjugated polymers developed for ion sensing demonstrated cation-induced aggregation with divalent cations [38], where quenching is enhanced with cation concentration increase [41]. Fluorescent carboxylated polyelectrolytes, water-soluble poly( $p$-phenylene ethylene) [42] and poly(thiophene) [43], are credited for their high sensitivity in biosensing applications. Sensing by these polymers of viologen and protein calmodulin, respectively, is based upon $\mathrm{Ca}^{2+}$ binding, which gives a shift in absorption or emission spectra based on aggregation of polymer or change in conformation.

In our recent report [13], we described the synthesis of water-soluble poly(phenylene vinylene)s (PPVs) having both carboxylic acid and methoxyoligoethylene glycol pendant groups (Scheme 1). In the current work, we focused our attention to the multiresponsive sensing behavior of these materials, as the carboxylic acid moiety should bring $\mathrm{pH}$ responsiveness while the oligoethylene glycol side chains may induce thermoresponsive behavior. Furthermore, the conjugated polymer backbone may enable a direct visual output signal upon a change in solubility and/or aggregation state of the polymer. Thermoresponsive oligoethylene glycol-modified polymers are gaining increased attention as alternatives for the well-known poly( $N$-isopropylacrylamide) (PNIPAAM) [44-46]. Such oligoethylene glycol-modified polymers often exhibit lower critical solution temperature (LCST) behavior in water, meaning that they are water-soluble at lower temperatures and undergo entropy-driven phase separation at a critical temperature. Opposite thermoresponsive behavior, so-called upper critical solution temperature (UCST), whereby the polymer is insoluble at lower temperatures and solubilizes upon heating, is less common in water and requires strong interpolymer interactions $[47,48]$. UCST behavior can also be induced by combining charged polymers with oppositely charged species, such as metal ions, metal ligand complexes, or organic compounds. A prime example is the UCST behavior of alginate in presence of calcium(II) ions [49-54]. Inspired by these systems, we additionally explored whether the acid-functionalized PPVs under study exhibit UCST behavior in presence of calcium(II) analogs (Scheme 1). 


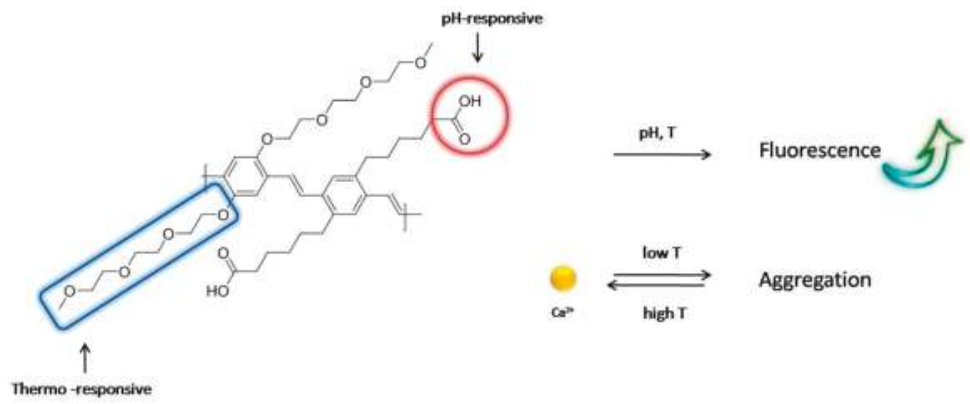

Scheme 1. General polymer structure with stimuli-responsive functional units, its response to $\mathrm{pH}$, temperature, and upper critical solution temperature (UCST)-like behavior when complexed with $\mathrm{Ca}^{2+}$.

\section{Experimental}

\subsection{Materials}

All chemicals were commercially available and used as received unless otherwise stated. Milli-Q water was obtained from a Sartorius Arium 611(Brussels, Belgium) with a Sartopore 2150 $(0.45+0.2 \mathrm{~mm}$ pore size) cartridge filter (resistivity less than $18.2 \mathrm{MU} \mathrm{cm}) . \mathrm{NaOH}$ and $\mathrm{HCl}$ are from Acros Organics (Geel, Belgium) and were diluted with Milli-Q water. Functionalized PPVs were synthesized as previously reported by us [13].

\subsection{Instrumentation/Methods}

The $\mathrm{pH}$ was recorded with a Mettler Toledo FE20 FiveEasy Benchtop pH meter (Brussels, Belgium). All spectroscopic measurements were carried out in $1 \mathrm{~cm}$ quartz cuvettes. The fluorescence emission spectra were measured on a Cary Eclipse fluorescence spectrometer (Santa Clara, CA, USA) with Peltier temperature control under stirring. The excitation wavelength was set at $392 \mathrm{~nm}$ with photomultiplier tube voltage at $600 \mathrm{~V}$. The split width of the excitation and emission were both $5 \mathrm{~nm}$. The fluorescence spectra were recorded with a recording emission range of 400-750 nm. The thermal measurements were taken between 5 and $85^{\circ} \mathrm{C}$ with heating/cooling rate of $1{ }^{\circ} \mathrm{C} / \mathrm{min}$. Optical absorption (UV-vis) spectra were measured using a Varian Cary 300 Bio UV-visible spectrometer (Santa Clara, CA, USA) equipped with a Cary Peltier temperature control while stirring. Samples were measured in quartz cuvettes with a pathlength of $1.0 \mathrm{~cm}$ in the wavelength range of $200-600 \mathrm{~nm}$. The concentration of each sample was $1.0 \mathrm{mg} / \mathrm{mL}$ in Milli-Q water.

${ }^{1} \mathrm{H}-\mathrm{NMR}$ spectra were recorded on a Bruker Avance 400 or $300 \mathrm{MHz}$ spectrometer (Billerica, MA, USA) at room temperature in deuterated solvents. Chemical shifts $(\delta)$ are given in ppm relative to TMS.

Size-exclusion chromatography (SEC) was performed on an Agilent 1260-series HPLC system (Santa Clara, CA, USA) equipped with a 1260 online degasser, a 1260 ISO-pump, a 1260 automatic liquid sampler, a thermostatted column compartment, a 1260 diode array detector (DAD) and a 1260 refractive index detector (RID). Analyses were performed on a PSS Gram30 column in series with a PSS Gram 1000 column at $50{ }^{\circ} \mathrm{C}$. DMAc containing $50 \mathrm{mM}$ of $\mathrm{LiCl}$ was used as eluent at a flow rate of $0.6 \mathrm{~mL} / \mathrm{min}$. The SEC traces were analyzed using the Agilent Chemstation software with the GPC add-on. Size exclusion chromatography was used to evaluate the number average molecular weight $\left(M_{\mathrm{n}}\right)$ and dispersity $(\bigoplus)$ against PMMA standards.

Turbidity measurements were performed on a Crystal16 from Technobis Crystallization Systems (Alkmaar, The Netherlands) at a wavelength of $600 \mathrm{~nm}$. The samples were fully dissolved at $\mathrm{pH}=10(0.5 \mathrm{mg} / \mathrm{mL})$, after which the samples were placed in the instrument and cooled to $10{ }^{\circ} \mathrm{C}$. The transmittance was measured during at least two controlled cooling/heating cycles with a cooling/heating rate of $1^{\circ} \mathrm{C} \cdot \mathrm{min}^{-1}$ while stirring in PS cuvettes controlled by block temperature probe. 


\subsection{Synthesis and Characterization}

\subsubsection{Synthesis of 1,4-bis(2-(2-(2-Methoxyethoxy)ethoxy)ethoxy)-2,5-divinylbenzene}

To a solution of 1,4-bis(2-(2-(2-methoxyethoxy)ethoxy)ethoxy)-2,5-diiodobenzene ( $1 \mathrm{~g}, 1.5 \mathrm{mmol})$ and vinyltributyltin $(1 \mathrm{~mL}, 3.7 \mathrm{mmol})$ in DMF $(6 \mathrm{~mL})$, triphenylphosphine palladium $(0)$ was added $(0.08 \mathrm{~g}, 0.07 \mathrm{mmol})$ and the reaction vessel was immediately sealed and degassed via freeze-pump-thaw (ca. 5 cycles). The mixture was then heated to $100{ }^{\circ} \mathrm{C}$ and stirred for $6 \mathrm{~h}$. The solution was then allowed to cool before diluting with DCM (ca. $50 \mathrm{~mL}$ ) and filtering into cold water. The organic layer was then washed three times with water, once with brine solution, dried $\left(\mathrm{Na}_{2} \mathrm{SO}_{4}\right)$, and reduced via vacuo. This was then purified by passing through a hexane column to yield the title compound, in approximately $40 \%$ yield, as a dark red oil.

$\delta \mathrm{H}\left(300 \mathrm{MHz} ; \mathrm{CDCl}_{3} ; \mathrm{Me}_{4} \mathrm{Si}\right): 7.25(2 \mathrm{H}, \mathrm{s}, \mathrm{ArH}), 4.18\left(4 \mathrm{H}, \mathrm{t}, J=6 \mathrm{~Hz}, \mathrm{CH}_{2}\right), 3.82(4 \mathrm{H}, \mathrm{t}, J=6 \mathrm{~Hz}$, $\left.\mathrm{CH}_{2}\right), 3.80-3.75\left(4 \mathrm{H}, \mathrm{m}, \mathrm{CH}_{2}\right), 3.74-3.68\left(8 \mathrm{H}, \mathrm{m}, \mathrm{CH}_{2}\right), 3.66-3.49\left(4 \mathrm{H}, \mathrm{m}, \mathrm{CH}_{2}\right), 3.38\left(6 \mathrm{H}, \mathrm{s}, \mathrm{OCH}_{3}\right)$.

IR: vmax(neat)/ $\mathrm{cm}^{-1} ; 2875(\mathrm{CH}$, aromatic), $1352(\mathrm{C}-\mathrm{O}$, ether), $1242(\mathrm{C}-\mathrm{O}$, ether), $1176(\mathrm{C}-\mathrm{O}$, ether), 1096 (C-O, ether) $395(\mathrm{C}-\mathrm{I}) . \mathrm{m} / z(\mathrm{CI}+) 677$ (MH+, 100\%). High Resolution (CI+): found (MH+): $677.0100 \mathrm{C}_{22} \mathrm{H}_{31} \mathrm{O}_{8} \mathrm{I}_{2}$ requires 677.0103. The $1 \mathrm{H}$ NMR data was in agreement with literature values [13].

\subsubsection{Synthesis of PMEE-PDTriG (Figure 1)}

A solution of tri- $n$-butylamine $(0.60 \mathrm{~mL}, 1.86 \mathrm{mmol})$, palladium acetate $(15.00 \mathrm{mg}, 0.02 \mathrm{mmol})$, tri-o-tolylphosphine (80.00 mg, $0.25 \mathrm{mmol})$, 1,4-bis(2-(2-(2-methoxyethoxy)ethoxy)ethoxy)-2,5divinylbenzene $(0.2 \mathrm{~g}, 0.44 \mathrm{mmol})$ and 1,4-diiodo-2,5-dimethoxybenzene $(0.36 \mathrm{mmol}), 6,6^{\prime}$-((2,5-diiodo1,4-phenylene)bis(oxy))dihexanoic acid $(0.055 \mathrm{~g}, 0.09 \mathrm{mmol})$ in DMF $(4 \mathrm{~mL})$ under an atmosphere of nitrogen, was degassed using freeze-thaw cycles $(\times 5)$, before heating at $90{ }^{\circ} \mathrm{C}$ for $24 \mathrm{~h}$. The mixture was then filtered and dissolved in basic water, this was then dialyzed against deionized water for 2 days with a 6-8 kD MWCO cellulose membrane. Water was removed in vacuum to give the title compound PMEE-PDTriG, in approximately 50\% yield, as a red gel-like solid.

$\delta \mathrm{H}\left(400 \mathrm{MHz} \mathrm{CDCl}_{3} ; \mathrm{Me}_{4} \mathrm{Si}\right): 7.96(2 \mathrm{H}, \mathrm{s}, \mathrm{ArH}), 7.54-7.51(2 \mathrm{H}, \mathrm{m}, \mathrm{ArH}), 7.29-7.20$ (4H, m, ArH), 6.92-6.85 (8H, m, CH=CH), $4.24\left(8 \mathrm{H}, \mathrm{m}, \mathrm{OCH}_{2}\right), 3.92\left(6 \mathrm{H}, \mathrm{bs}, \mathrm{OCH}_{3}\right), 3.69\left(6 \mathrm{H}, \mathrm{bs}, \mathrm{OCH}_{3}\right), 3.58-3.19$ $\left(\mathrm{m}, \mathrm{OCH}_{2}, \mathrm{OCH}_{3}\right), 2.29\left(4 \mathrm{H}, \mathrm{m}, \mathrm{CH}_{2}\right), 1.72-1.50\left(12 \mathrm{H}, \mathrm{m}, \mathrm{CH}_{2}\right)$.
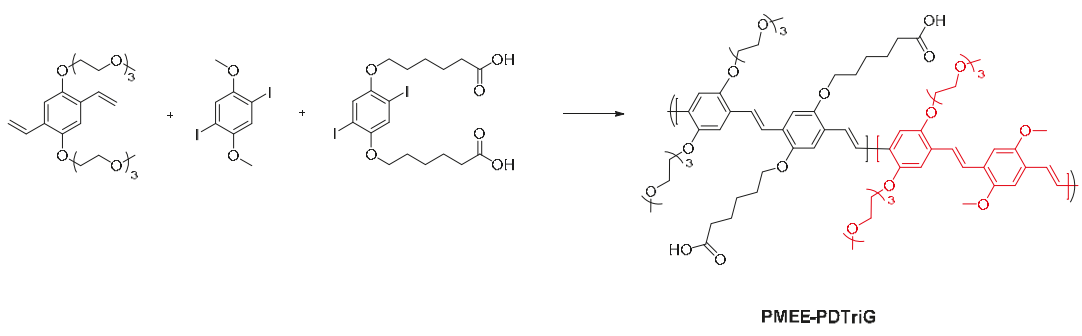

Figure 1. Synthetic scheme of PMEE-PDTriG.

\subsection{Sample Preparation}

Samples for fluorescent measurements first were dissolved in Milli-Q water at $\mathrm{pH}=13$ and adjusted to lower $\mathrm{pH}$ values by dilute $\mathrm{HCl}$. All samples were prepared at $0.01 \mathrm{mg} / \mathrm{mL}$. For UV-vis measurements, samples were prepared at $\mathrm{pH}=10(0.5 \mathrm{mg} / \mathrm{mL})$ in Milli-Q water (Milli-Q, resistivity $\leq 18.2 \mathrm{M} \Omega \cdot \mathrm{cm})$. 


\section{Results and Discussion}

\subsection{Synthesis and Characterization}

PPVs with functional carboxylic acid and oligoethylene glycol units were previously reported to show high solubility and high luminescence in aqueous and organic media [13]. Here, specifically 2,5-substituted PPV polymers decorated with methoxytriethylene glycol units and carboxylic acid groups (denoted as PDTriG) and its more hydrophobic copolymer with methoxytrietylene glycol and methoxy side groups (denoted as PMEE-PDTriG) were investigated to evaluate their responsive behavior (Figure 2). PDTriG was synthesized as previously reported [13] whereas the monomer and polymer of PMEE-PDTriG were synthesized using a slightly modified literature procedure [13]. These polymers were characterized by means of NMR and SEC. Size exclusion chromatography (SEC) with DMA as a solvent and PMMA as calibration standard was used to obtain the average molecular mass of PDTriG $\left(M_{n}=197,000 ; \emptyset=1.34\right)$ and PMEE-PDTriG $\left(M_{n}=108,200 ; \emptyset=1.04\right)$.

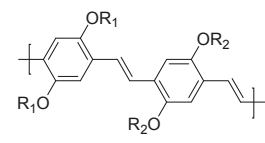

PDTriG

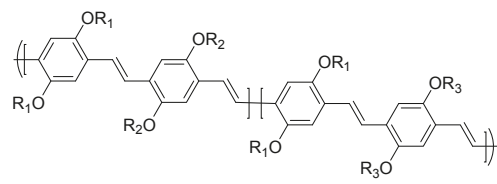

PMEE-PDTriG

$\mathrm{R} 1=\mathrm{CH}_{2} \mathrm{CH}_{2} \mathrm{OCH}_{2} \mathrm{CH}_{2} \mathrm{OCH}_{2} \mathrm{CH}_{2} \mathrm{OCH}_{3}$ $\mathrm{R}_{2}=\mathrm{C}_{5} \mathrm{H}_{10} \mathrm{CO}_{2} \mathrm{H}$ $\mathrm{R}_{3}=\mathrm{CH}_{3}$

Figure 2. Structures of the polymers: PDTriG and PMEE-PDTriG.

\subsection{Responsive Behavior}

\subsection{1. $\mathrm{pH}$-Responsive Behavior}

It is well known that the optical properties of CPs are governed by the polarity of the polymer, conformational changes in the backbone, the polarity of the solvent, intramolecular dynamics, and interchain interactions $[55,56]$. A change in the conformation of the polymer chain is dictated by different variables, among which solvent and temperature are prominent. Accordingly, any external factor effecting backbone configuration influences the photophysical features of CPs. It was shown previously that the emission spectra of functional PPVs were highly dependent on acidity of the media [13]. Photophysical properties of both PDTriG and PMEE-PDTriG were studied in aqueous media as function of $\mathrm{pH}$ by fluorescence spectroscopy. It was observed that the polymers did not readily dissolve in water and in buffer solution, unless the $\mathrm{pH}$ was increased to 13 . Therefore, the samples were first fully solubilized at $\mathrm{pH}=13$ followed by addition of dilute hydrochloric acid solution to adjust the $\mathrm{pH}$ to the desired $\mathrm{pH}$ values. When following this procedure the polymers remained in aqueous solution and no macroscopic precipitate was observed. As shown in the fluorescence spectra in Figure 3a, PDTriG has a maximum emission at $498 \mathrm{~nm}$ and an excitation maximum at $392 \mathrm{~nm}$, as measured at $\mathrm{pH}=13,0.01 \mathrm{mg} / \mathrm{mL}$. PMEE-PDTriG was found to have an emission $\lambda_{\max }$ of $478 \mathrm{~nm}$ and an excitation $\lambda_{\max }$ of $378 \mathrm{~nm}$ when measured at the same concentration and $\mathrm{pH}$ (Figure $3 \mathrm{~b}$ ) indicating both polymers have similar optical properties. Thus, all the emission measurements were carried out at an excitation maximum wavelength $\lambda_{\max }=392 \mathrm{~nm}$ for PDTriG and $\lambda_{\max }=378 \mathrm{~nm}$ for PMEE-PDTriG. The fluorescence intensity of both polymers was found to be dependent on the $\mathrm{pH}$ of the solution, as expected based on the presence of the carboxylic acid groups. While at $\mathrm{pH}$ below 7 quenching of the fluorescence was observed, strong emission was found at higher $\mathrm{pH}(\mathrm{pH}>7)$. This change in emission intensity can be attributed to the enhanced solubility of the polymer at higher $\mathrm{pH}$ values due to deprotonation of the carboxylic acid groups, thereby suppressing aggregation of the polymer and quenching of fluorescence [57]. Fluorescence intensity increased for both of the polymers 
as $\mathrm{pH}$ values increased, without shifting of emission maxima peaks (Figure $3 \mathrm{c}, \mathrm{d}$ ). A slight blue shift is observed for PDTriG with decreasing acidity, at pH 6 and below, due to aggregation of amphiphilic CPs as they become protonated [56]. It was, however, observed that the fluorescence intensity of PDTriG decreased in time at pH 13 and at pH 6 and lower. In contrast, PMEE-PDTriG was found to be more stable and only showed a decrease in fluorescence intensity at pH 13 and below pH 3. At lower $\mathrm{pH}$ values, protonation of the carboxylic acid groups is presumed to cause insolubility of the polymers; this was proved by complete loss of emission intensity, which was not restored even after adjusting $\mathrm{pH}$ value back to 10. Especially, the higher stability at lower $\mathrm{pH}$ values for PMEE-PDTriG indicates that the presence of protonated carboxylic acid groups induces degradation and/or insolubility, which is more pronounced for PDTriG as it has more carboxylic acid groups. Note that for sample preparation, the polymers were only dissolved in $\mathrm{pH} 13$ for a short time to avoid degradation of the materials, presumably resulting from the high concentration of hydroxyl anions facilitating degradation of the polymer backbone.
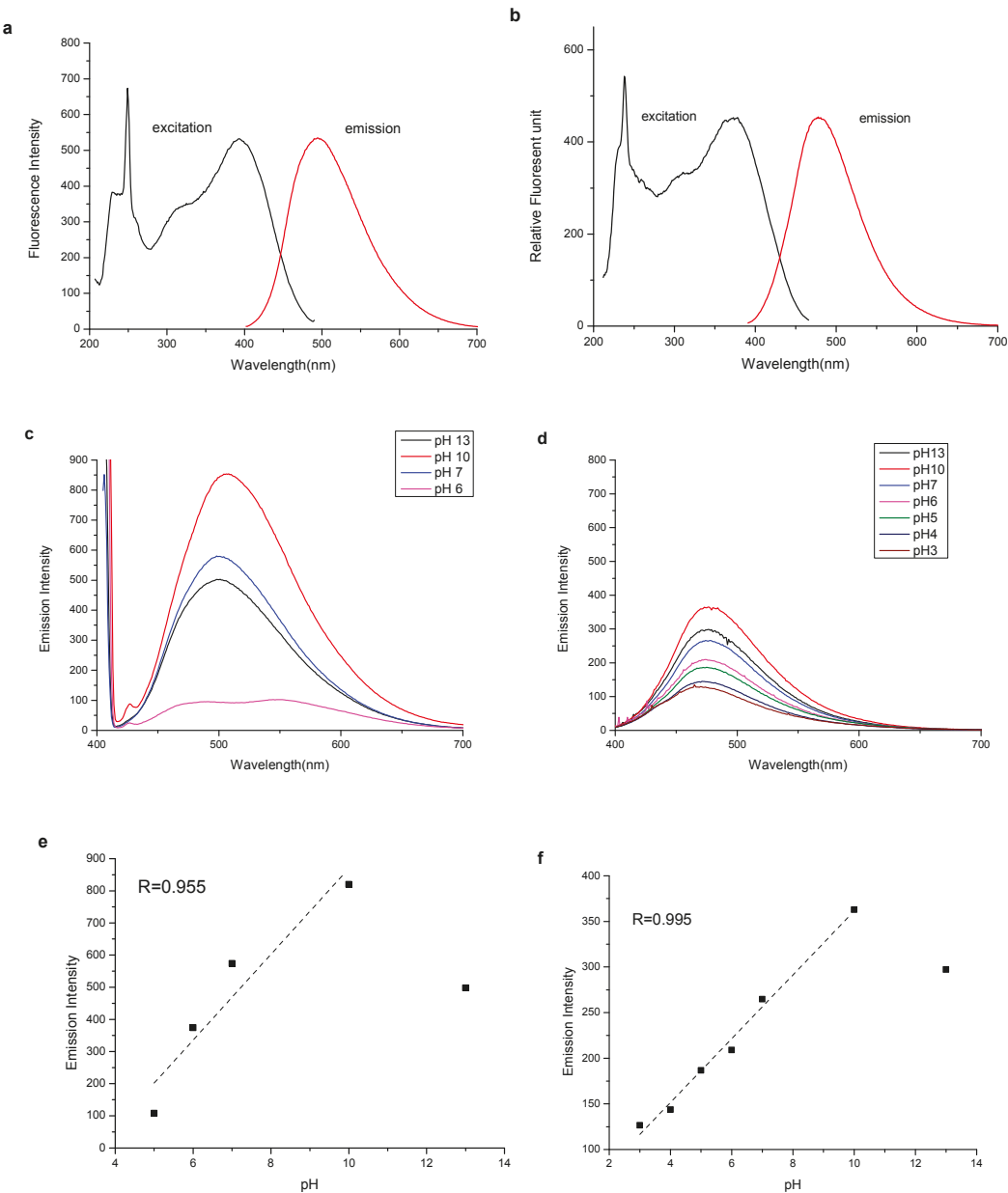

Figure 3. Emission and excitation spectra of (a) PDTriG; (b) PMEE-PDTriG, measured at pH =13, $0.01 \mathrm{M}$. Fluorescence spectra of (c) PDTriG; (d) PMEE-PDTriG at different $\mathrm{pH}$ values at $20{ }^{\circ} \mathrm{C}, 0.01 \mathrm{mg} / \mathrm{mL}$. Linear fit of fluorescence intensity of (e) PDTriG (f) PMEE-PDTriG as a function of $\mathrm{pH}$ at emission $\lambda_{\max }$. 
When plotting the fluorescence intensity of PDTriG versus $\mathrm{pH}$, it is clear that no good correlation can be drawn, possibly due to the low stability of the polymers. However, PMEE-PDTriG revealed a linear decrease of fluorescence intensity with decreasing $\mathrm{pH}$ in between $\mathrm{pH} 3$ and $\mathrm{pH} 10$, making it suitable as fluorescent $\mathrm{pH}$ sensor. This rather broad $\mathrm{pH}$ range also facilitates employment in a biological environment as it covers the physiological $\mathrm{pH}$ range.

\subsubsection{Thermoresponsive Behavior}

As mentioned above, the conformation of the $\pi$-conjugated backbone has substantial impact on the photophysical properties of CPs [8]. Therefore, thermoresponsive behavior of the studied PPVs would also induce a change in solubility that also strongly influences the backbone conformation, directly leading to fluorescent output signal. Possible temperature effects on the photoluminescent properties of the more stable PMEE-PDTriG were studied by investigating the fluorescence intensity as function of temperatures, where it was anticipated that these polymers may exhibit LCST behavior based on the methoxyoligoethylene glycol side chains. Fluorescence intensity of the PMEE-PDTriG was measured at different $\mathrm{pH}$ values upon heating from 20 to $80^{\circ} \mathrm{C}$, revealing a minor decrease in fluorescence intensity with increasing temperature due to increased chain mobility leading to a decrease in conjugation length (Figure 4). The relative order in fluorescence intensity at different $\mathrm{pH}$ values is, however, retained at different temperatures. Nonetheless, this minor temperature influence will make it necessary to recalibrate the $\mathrm{pH}$-sensing response at different temperatures.

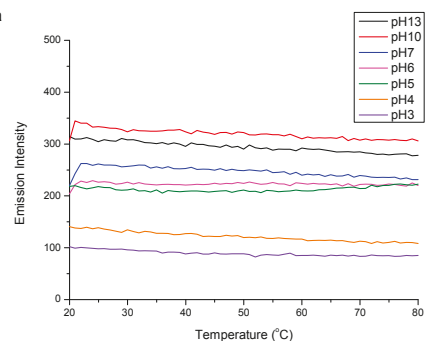

c

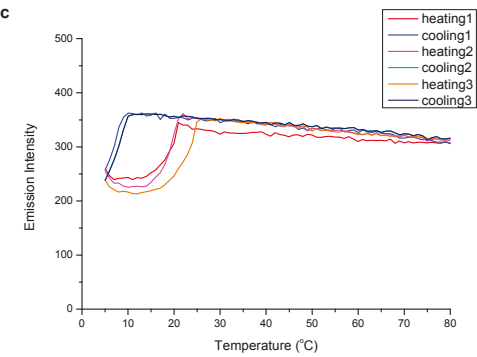

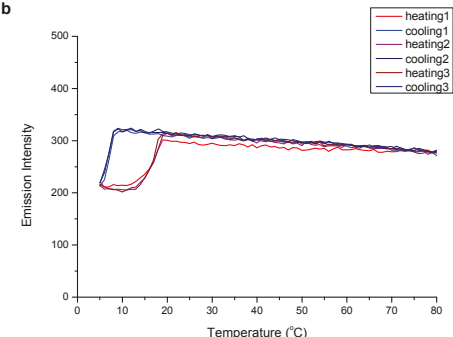

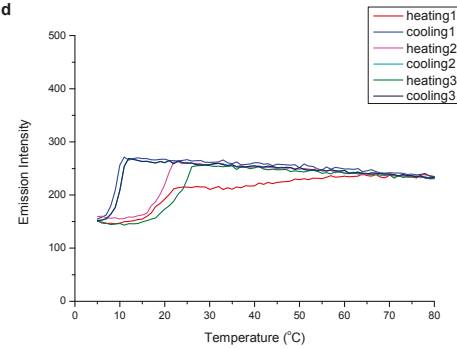

Figure 4. Fluorescence emission as a function of temperature at different $\mathrm{pH}$ values (a) PMEE-PDTriG. Thermal stability with heating and cooling cycles of PMEE-PDTriG at (b) $\mathrm{pH}=13$; (c) $\mathrm{pH}=10$; (d) $\mathrm{pH}=7$.

A more critical look at Figure 4 reveals some minor disturbances in fluorescence intensity at lower temperature, especially at $\mathrm{pH}$ 7. Intrigued by this effect and to probe the stability of the polymer, the fluorescence intensity was recorded during multiple heating-cooling cycles at $\mathrm{pH} 7,10$, and 13 in between 10 and $80^{\circ} \mathrm{C}$ (Figure $4 \mathrm{~b}-\mathrm{d}$ ). First of all, these experiments revealed that PMEE-PDTriG is stable during multiple cycles; only the first heating shows a slightly different behavior at $\mathrm{pH} 7$ and 10, most likely related to solubilization since this effect is no longer seen in the second and third heating runs. 
Secondly, it is evident that PMEE-PDTriG reveals distinct thermoresponsive behavior around $10-20{ }^{\circ} \mathrm{C}$. This abrupt increase in fluorescence emission intensity upon heating is most likely due to LCST-like behavior of the oligoethylene glycol side chains, as such LCST behavior is an abrupt cooperative dehydration effect. More specifically, the observed increase in fluorescence emission intensity may be attributed to (partial) dehydration of the oligoethylene glycol side chains, leading them to collapse. No macroscopic phase separation is observed, as the PMEE-PDTriG remains stabilized and solubilized by the charged carboxylate groups. Interestingly, the reverse process of rehydration of the oligoethylene glycol side chains only occurs around $10^{\circ} \mathrm{C}$, representing some hysteresis in the responsive behavior. This observation may be related to the presence of the hydrophobic alkyl chains on the carboxylic acid side chains facilitating the formation of hydrophobic domains with the dehydrated oligoethylene glycol chains, thereby making it more difficult to rehydrate these oligoethylene glycol chains during cooling.

To gain further insights into the effect of temperature and, especially, dehydration of the oligoethylene glycol side chains on the polymer chain conformation, full emission spectra were recorded at different temperatures (Figure 5). Changing the temperature, however, showed no shift in emission wavelength maxima but only resulted in a decrease in fluorescence intensity. The low emission intensity at lower temperatures showed a pronounced increase at $9^{\circ} \mathrm{C}$, giving a jump of emission intensity as depicted in Figure 5b. Therefore, it may be speculated that the collapse of the oligoethylene glycol side chains leads to an increase in chain rigidity, thereby increasing the conjugation length that results in enhanced emission intensity. It is noteworthy to mention that the more hydrophilic PDTriG did not show such thermoresponsive behavior.

a

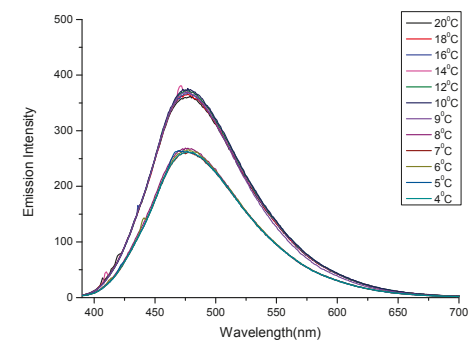

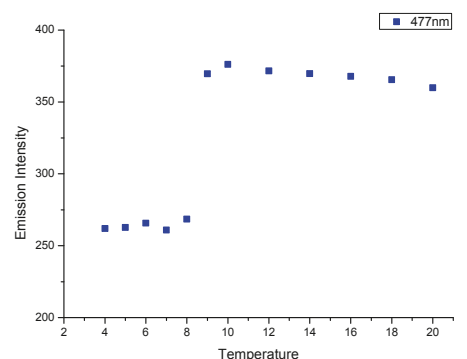

Figure 5. (a) Fluorescence emission spectra of PMEE-PDTriG at low temperatures, from 4 to $20{ }^{\circ} \mathrm{C}$; (b) emission intensity at emission maxima, $\lambda_{\max }$ versus temperature plot.

The observed thermoresponsive emission behavior of PMEE-PDTriG may be utilized for aqueous temperature sensing, whereby the hysteresis gap could lead to a thermal memory of the solution temperature $[58,59]$.

\subsubsection{UCST-Like Behavior with Calcium(II) Ions}

Besides studying the potential of these functional PPVs as $\mathrm{pH}$ and temperature sensors, we were interested to exploit the presence of carboxylate groups for inducing UCST-like thermoresponsive solubility behavior. Therefore, PDTriG was employed as it has a higher load of carboxylic acid groups. The polymer was solubilized in basic solution so that the carboxylic acid groups were present as carboxylate groups, which are known to bind with calcium(II) ions in a 2:1 fashion [60-62]. Upon addition of half an equivalent of calcium(II) ions compared to the carboxylate groups, all polymer precipitated out of solution, ascribed to interpolymer crosslinking by calcium(II)-carboxylate coordination as schematically depicted in Scheme 2. By heating up the polymer-calcium(II) precipitate, the supramolecular association is weakened leading to dissolution of the polymer and the calcium(II) ions, thereby providing reversible binding of calcium(II) as function of temperature. 


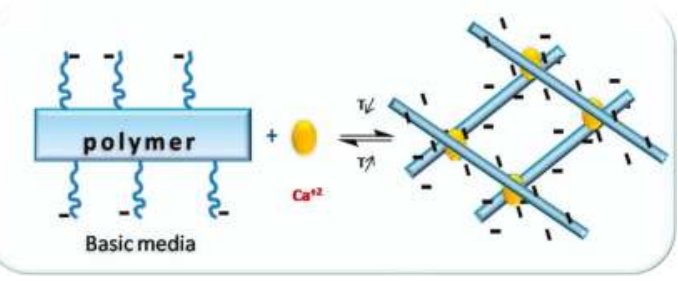

Scheme 2. Schematic representation of the reversible binding of PDTriG with $\mathrm{CaCl}_{2}$ in basic media leading to UCST behavior.

The complexation and precipitation of the polymer with calcium(II) ions is also shown in Figure 6, indicated by the loss of UV-absorbance as well as by the photographs of the vial. As the precipitated PDTriG-calcium complex is held together by noncovalent supramolecular interactions, we explored whether heating would lead to sufficient lowering of the interaction strength to bring the polymer back in solution. Indeed, heating the sample vial led to regaining of the yellow solution as the precipitate redissolved. Subsequent cooling led to precipitation demonstrating the reversibility of this UCST-like thermoresponsive behavior of PDTriG. Temperature response of the PDTriG and PDTriG-calcium complex is further shown by UV-vis turbidimetry. As depicted in Figure 7, heating the samples from 10 to $70^{\circ} \mathrm{C}$ gives no change in the transmittance of the polymer solution, whereas the polymer-calcium complex goes through UCST-like phase transition.
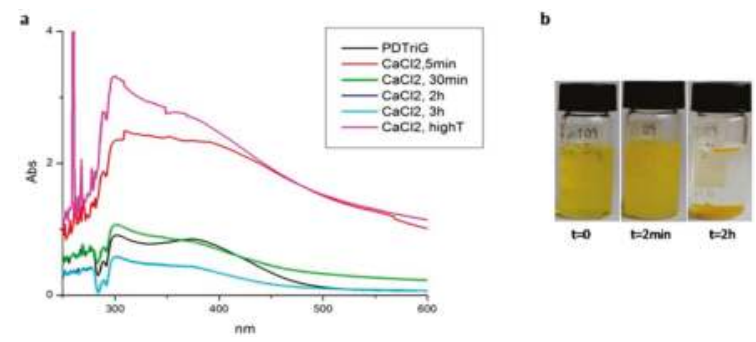

Figure 6. (a) UV-vis spectra of PDTriG $\left(\mathrm{pH}=10,0.5 \mathrm{mg} / \mathrm{mL}\right.$ ) on addition of $\mathrm{CaCl}_{2}$ (1:1 equivalents) in basic media at $25^{\circ} \mathrm{C}$ after $5 \mathrm{~min}$ (red), after $30 \mathrm{~min}$ (green), after $2 \mathrm{~h}$ (blue), after $3 \mathrm{~h}$ (light blue), and at $60{ }^{\circ} \mathrm{C}$ (purple); (b) visual observation of complexation within time demonstrating the phase separation of PDTriG in presence of calcium(II) ions.

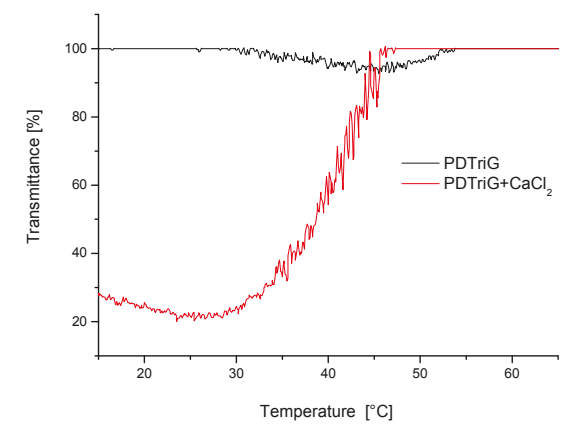

Figure 7. Transmittance as a function of temperature for PDTriG (black) and PDTriG with $\mathrm{CaCl}_{2}$ (red) at $\mathrm{pH}=10(0.5 \mathrm{mg} / \mathrm{mL})$ measured at a wavelength of $600 \mathrm{~nm}$. 


\section{Conclusions}

Functional groups on PPV not only give high solubility in water but also provide new properties for sensing regime of the polymers. In this study, water-soluble PPVs with carboxylic acid groups and methoxyoligoethylene units as pendant side chains are reported to have high luminescence in aqueous media with multiresponsive behavior. Higher solubility is attained for the polymers with increased $\mathrm{pH}$ values as more carboxylic acid groups are deprotonated, thereby inhibiting aggregation of the polymers and enhancing fluorescence intensity. This phenomenon is observed for both of the polymers, providing $\mathrm{pH}$ response behavior of PDTriG in between $\mathrm{pH}$ 6-13 and in between $\mathrm{pH}$ 3-13 for PMEE-PDTriG. Higher solubility of the reported polymers was also achieved by influence of temperature, making them polymeric-thermometers as they are thermosensitive in aqueous media. Photophysical properties at different $\mathrm{pH}$ values in a broad temperature window $\left(10-80^{\circ} \mathrm{C}\right)$ shows that there is a temperature response for both polymers. Moreover, stability of the polymers was maintained throughout multiple heating-cooling cycles. Furthermore, UCST-like thermoresponsive behavior was demonstrated for PDTriG with $\mathrm{Ca}^{2+}$ ions, which are known to bind divalently with carboxylate groups. The combined $\mathrm{pH}$-responsive and thermoresponsive properties of these PPVs are promising features for the design of sensors, specifically for their use as dual sensors for temperature and $\mathrm{pH}$ values. Such dual sensors are a highly desired addition to the class of polymeric sensors and a related system based on a temperature-responsive polymer with a $\mathrm{pH}$-responsive solvatochromic dye in the side chain. The hysteresis gap in the heating-cooling cycles at lower temperatures for PMEE-PDTriG brings about an optional application of a solution thermometer with memory function.

Acknowledgments: Kanykei Ryskulova and Richard Hoogenboom thank Ghent University (BOF) and FWO Flanders for financial support. Richard Hoogenboom is grateful to BELSPO (IAP VII/5 Functional Supramolecular Systems FS2). Thomas Kerr-Phillips thanks MacDiarmid Institute for Advanced Materials and Nanotechnology for doctoral scholarship.

Author Contributions: Jadranka Travas-Sejdic and Richard Hoogenboom conceived and designed the experiments and supervised the work; David Barker and Hui Peng designed the polymers and David Barker supervised the polymer synthesis; Anupama Rao Gulur Srinivas and Thomas Kerr-Phillips synthesized the polymers; Kanykei Ryskulova performed the experiments and analyzed the data; Kanykei Ryskulova and Richard Hoogenboom wrote the paper.

Conflicts of Interest: The authors declare no conflict of interest.

\section{References}

1. Schmaljohann, D. Thermo- and pH-responsive polymers in drug delivery. Adv. Drug Deliv. Rev. 2006, 58, 1655-1670. [CrossRef] [PubMed]

2. Mura, S.; Nicolas, J.; Couvreur, P. Stimuli-responsive nanocarriers for drug delivery. Nat. Mater. 2013, 12, 991-1003. [CrossRef] [PubMed]

3. Yin, L.Y.; He, C.S.; Huang, C.S.; Zhu, W.P.; Wang, X.; Xu, Y.F.; Qian, X.H. A dual pH and temperature responsive polymeric fluorescent sensor and its imaging application in living cells. Chem. Commun. 2012, 48, 4486-4488. [CrossRef] [PubMed]

4. Bao, Y.Y.; De Keersmaecker, H.; Corneillie, S.; Yu, F.; Mizuno, H.; Zhang, G.F.; Hofkens, J.; Mendrek, B.; Kowalczuk, A.; Smet, M. Tunable ratiometric fluorescence sensing of intracellular pH by aggregation-induced emission-active hyperbranched polymer nanoparticles. Chem. Mater. 2015, 27, 3450-3455. [CrossRef]

5. Peng, H.S.; Stolwijk, J.A.; Sun, L.N.; Wegener, J.; Wolfbeis, O.S. A nanogel for ratiometric fluorescent sensing of intracellular pH values. Angew. Chem. Int. Ed. 2010, 49, 4246-4249. [CrossRef] [PubMed]

6. Li, K.; Liu, B. Water-soluble conjugated polymers as the platform for protein sensors. Polym. Chem. 2010, 1, 252-259. [CrossRef]

7. Pietsch, C.; Hoogenboom, R.; Schubert, U.S. Soluble polymeric dual sensor for temperature and $\mathrm{pH}$ value. Angew. Chem. Int. Ed. 2009, 48, 5653-5656. [CrossRef] [PubMed]

8. Zhu, C.; Liu, L.; Yang, Q.; Lv, F.; Wang, S. Water-soluble conjugated polymers for imaging, diagnosis, and therapy. Chem. Rev. 2012, 112, 4687-4735. [CrossRef] [PubMed] 
9. Cabane, E.; Zhang, X.Y.; Langowska, K.; Palivan, C.G.; Meier, W. Stimuli-responsive polymers and their applications in nanomedicine. Biointerphases 2012, 7, 9. [CrossRef] [PubMed]

10. Cheng, R.; Meng, F.H.; Deng, C.; Klok, H.A.; Zhong, Z.Y. Dual and multi-stimuli responsive polymeric nanoparticles for programmed site-specific drug delivery. Biomaterials 2013, 34, 3647-3657. [CrossRef] [PubMed]

11. Gil, E.S.; Hudson, S.M. Stimuli-reponsive polymers and their bioconjugates. Prog. Polym. Sci. 2004, 29, 1173-1222. [CrossRef]

12. Galaev, I.Y.; Mattiasson, B. "Smart" polymers and what they could do in biotechnology and medicine. Trends Biotechnol. 1999, 17, 335-340. [CrossRef]

13. Gulur Srinivas, A.R.; Kerr-Phillips, T.E.; Peng, H.; Barker, D.; Travas-Sejdic, J. Water-soluble anionic poly( $p$-phenylene vinylenes) with high luminescence. Polym. Chem. 2013, 4, 2506-2514. [CrossRef]

14. Enzenberg, A.; Laschewsky, A.; Boeffel, C.; Wischerhoff, E. Influence of the near molecular vicinity on the temperature regulated fluorescence response of poly(N-vinylcaprolactam). Polymers 2016, 8, 109. [CrossRef]

15. Zhang, Q.L.; Vancoillie, G.; Mees, M.A.; Hoogenboom, R. Thermoresponsive polymeric temperature sensors with broad sensing regimes. Polym. Chem. 2015, 6, 2396-2400. [CrossRef]

16. Pietsch, C.; Vollrath, A.; Hoogenboom, R.; Schubert, U.S. A fluorescent thermometer based on a pyrene-labeled thermoresponsive polymer. Sensors 2010, 10, 7979-7990. [CrossRef] [PubMed]

17. Uchiyama, S.; Kawai, N.; De Silva, A.P.; Iwai, K. Fluorescent polymeric AND logic gate with temperature and $\mathrm{pH}$ as inputs. J. Am. Chem. Soc. 2004, 126, 3032-3033. [CrossRef] [PubMed]

18. Jin, Q.A.; Liu, G.Y.; Ji, J.A. Micelles and reverse micelles with a photo and thermo double-responsive block copolymer. J. Polym. Sci. Polym. Chem. 2010, 48, 2855-2861. [CrossRef]

19. Uchiyama, S.; Makino, Y. Digital fluorescent $\mathrm{pH}$ sensors. Chem. Commun. 2009, 19, 2646-2648. [CrossRef] [PubMed]

20. Korostynska, O.; Arshak, K.; Gill, E.; Arshak, A. Review on state-of-the-art in polymer based pH sensors. Sensors 2007, 7, 3027-3042. [CrossRef]

21. Wang, $\mathrm{S}$. Dopamine modified cationic conjugated polymers as a new platform for $\mathrm{pH}$ sensing and autophagy imaging. Adv. Funct. Mater. 2013, 23, 764-769.

22. Gota, C.; Uchiyama, S.; Ohwada, T. Accurate fluorescent polymeric thermometers containing an ionic component. Analyst 2007, 132, 121-126. [CrossRef] [PubMed]

23. Wang, X.-D.; Wolfbeis, O.S.; Meier, R.J. Luminescent probes and sensors for temperature. Chem. Soc. Rev. 2013, 42, 7834-7869. [CrossRef] [PubMed]

24. Khine, Y.Y.; Jiang, Y.Y.; Dag, A.; Lu, H.X.; Stenzel, M.H. Dual-responsive pH and temperature sensitive nanoparticles based on methacrylic acid and Di(ethylene glycol) methyl ether methacrylate for the triggered release of drugs. Macromol. Biosci. 2015, 15, 1091-1104. [CrossRef] [PubMed]

25. Wang, X.D.; Meier, R.J.; Wolfbeis, O.S. A fluorophore-doped polymer nanomaterial for referenced imaging of $\mathrm{pH}$ and temperature with sub-micrometer resolution. Adv. Funct. Mater. 2012, 22, 4202-4207. [CrossRef]

26. McQuade, D.T.; Pullen, A.E.; Swager, T.M. Conjugated polymer-based chemical sensors. Chem. Rev. 2000, 100, 2537-2574. [CrossRef] [PubMed]

27. Feng, X.L.; Liu, L.B.; Wang, S.; Zhu, D.B. Water-soluble fluorescent conjugated polymers and their interactions with biomacromolecules for sensitive biosensors. Chem. Soc. Rev. 2010, 39, 2411-2419. [CrossRef] [PubMed]

28. Aydemir, N.; Malmstrom, J.; Travas-Sejdic, J. Conducting polymer based electrochemical biosensors. Phys. Chem. Chem. Phys. 2016, 18, 8264-8277. [CrossRef] [PubMed]

29. Zhu, B.; Alsager, O.A.; Kumar, S.; Hodgkiss, J.M.; Travas-Sejdic, J. Label-free electrochemical aptasensor for femtomolar detection of $17 \beta$-estradiol. Biosens. Bioelectron. 2015, 70, 398-403. [CrossRef] [PubMed]

30. Liu, B.; Bazan, G.C. Homogeneous fluorescence-based DNA detection with water-soluble conjugated polymers. Chem. Mater. 2004, 16, 4467-4476. [CrossRef]

31. Gaylord, B.S.; Heeger, A.J.; Bazan, G.C. DNA detection using water-soluble conjugated polymers and peptide nucleic acid probes. Proc. Natl. Acad. Sci. USA 2002, 99, 10954-10957. [CrossRef] [PubMed]

32. Jadranka, T.-S.; AnupamaRaoGulur, S. Optical gene detection using conjugated polymers. In Optical Properties of Functional Polymers and Nano Engineering Applications; CRC Press: Boca Raton, FL, USA, 2014; pp. 61-86.

33. Srinivas, A.R.G.; Peng, H.; Barker, D.; Travas-Sejdic, J. Switch on or switch off: An optical DNA sensor based on poly( $p$-phenylenevinylene) grafted magnetic beads. Biosens. Bioelectron. 2012, 35, 498-502. [CrossRef] [PubMed] 
34. Srinivas, A.R.G.; Barker, D.; Travas-Sejdic, J. “Switch-on” DNA sensor based on poly (p-phenylene vinylenes) bound tentacle probes. Pure Appl. Chem. 2015, 87, 707-715. [CrossRef]

35. Giepmans, B.N.G.; Adams, S.R.; Ellisman, M.H.; Tsien, R.Y. Review-The fluorescent toolbox for assessing protein location and function. Science 2006, 312, 217-224. [CrossRef] [PubMed]

36. Fan, C.H.; Plaxco, K.W.; Heeger, A.J. High-efficiency fluorescence quenching of conjugated polymers by proteins. J. Am. Chem. Soc. 2002, 124, 5642-5643. [CrossRef] [PubMed]

37. Zhang, W.; Sun, H.; Yin, S.Y.; Chang, J.J.; Li, Y.H.; Guo, X.Y.; Yuan, Z. Bright red-emitting polymer dots for specific cellular imaging. J. Mater. Sci. 2015, 50, 5571-5577. [CrossRef]

38. Thomas, S.W.; Joly, G.D.; Swager, T.M. Chemical sensors based on amplifying fluorescent conjugated polymers. Chem. Rev. 2007, 107, 1339-1386. [CrossRef] [PubMed]

39. Lakowicz, J.R. Principles of Fluorescence Spectroscopy, 2nd ed.; Plenm: New York, NY, USA, 1999.

40. Tan, C.Y.; Alas, E.; Muller, J.G.; Pinto, M.R.; Kleiman, V.D.; Schanze, K.S. Amplified quenching of a conjugated polyelectrolyte by cyanine dyes. J. Am. Chem. Soc. 2004, 126, 13685-13694. [CrossRef] [PubMed]

41. Kim, I.B.; Dunkhorst, A.; Gilbert, J.; Bunz, U.H.F. Sensing of lead ions by a carboxylate-substituted PPE: Multivalency effects. Macromolecules 2005, 38, 4560-4562. [CrossRef]

42. Jiang, H.; Zhao, X.Y.; Schanze, K.S. Amplified fluorescence quenching of a conjugated polyelectrolyte mediated by $\mathrm{Ca}^{2+}$. Langmuir 2006, 22, 5541-5543. [CrossRef] [PubMed]

43. Nilsson, K.P.R.; Inganas, O. Optical emission of a conjugated polyelectrolyte: Calcium-induced conformational changes in calmodulin and calmodulin-calcineurin interactions. Macromolecules 2004, 37, 9109-9113. [CrossRef]

44. Vancoillie, G.; Frank, D.; Hoogenboom, R. Thermoresponsive poly(oligo ethylene glycol acrylates). Prog. Polym. Sci. 2014, 39, 1074-1095. [CrossRef]

45. Weber, C.; Hoogenboom, R.; Schubert, U.S. Temperature responsive bio-compatible polymers based on poly(ethylene oxide) and poly(2-oxazoline)s. Prog. Polym. Sci. 2012, 37, 686-714. [CrossRef]

46. Lutz, J.F. Thermo-switchable materials prepared using the OEGMA-platform. Adv. Mater. 2011, 23, $2237-2243$. [CrossRef]

47. Seuring, J.; Agarwal, S. Polymers with upper critical solution temperature in aqueous solution. Macromol. Rapid Commun. 2012, 33, 1898-1920. [CrossRef] [PubMed]

48. Zhang, Q.L.; Hoogenboom, R. Polymers with upper critical solution temperature behavior in alcohol/water solvent mixtures. Prog. Polym. Sci. 2015, 48, 122-142. [CrossRef]

49. Plamper, F.A.; Schmalz, A.; Ballauff, M.; Muller, A.H.E. Tuning the thermoresponsiveness of weak polyelectrolytes by $\mathrm{pH}$ and light: Lower and upper critical-solution temperature of poly(N,N-dimethylaminoethyl methacrylate). J. Am. Chem. Soc. 2007, 129, 14538-14539. [CrossRef] [PubMed]

50. Ott, C.; Hoogenboom, R.; Hoeppener, S.; Wouters, D.; Gohy, J.F.; Schubert, U.S. Tuning the morphologies of amphiphilic metallo-supramolecular triblock terpolymers: From spherical micelles to switchable vesicles. Soft Matter 2009, 5, 84-91. [CrossRef]

51. Jia, X.; Chen, D.Y.; Jiang, M. Preparation of PEO- $b$-P2VPH(+)- $\mathrm{S}_{2} \mathrm{O}_{8}{ }^{2-}$ micelles in water and their reversible UCST and redox-responsive behavior. Chem. Commun. 2006, 16, 1736-1738. [CrossRef] [PubMed]

52. Serp, D.; Mueller, M.; von Stockar, U.; Marison, I.W. Low-temperature electron microscopy for the study of polysaccharide ultrastructures in hydrogels. II. Effect of temperature on the structure of $\mathrm{Ca}^{2+}$-alginate beads. Biotechnol. Bioeng. 2002, 79, 253-259. [CrossRef] [PubMed]

53. Pawar, S.N.; Edgar, K.J. Alginate derivatization: A review of chemistry, properties and applications. Biomaterials 2012, 33, 3279-3305. [CrossRef] [PubMed]

54. Morch, Y.A.; Donati, I.; Strand, B.L.; Skjak-Braek, G. Effect of $\mathrm{Ca}^{2+}, \mathrm{Ba}^{2+}$, and $\mathrm{Sr}^{2+}$ on alginate microbeads. Biomacromolecules 2006, 7, 1471-1480. [CrossRef] [PubMed]

55. De Silva, A.P.; Gunaratne, H.Q.N.; Gunnlaugsson, T.; Huxley, A.J.M.; McCoy, C.P.; Rademacher, J.T.; Rice, T.E. Signaling recognition events with fluorescent sensors and switches. Chem. Rev. 1997, 97, 1515-1566. [CrossRef] [PubMed]

56. Van Severen, I.; Breselge, M.; Fourier, S.; Adriaensens, P.; Manca, J.; Lutsen, L.; Cleij, T.J.; Vanderzande, D. 2,5-substituted PPV-derivatives with different polarities: The effect of side chain polarity on solubility, optical and electronic properties. Macromol. Chem. Phys. 2007, 208, 196-206. [CrossRef]

57. Lee, K.; Kim, H.J.; Kim, J. Design principle of conjugated polyelectrolytes to make them water-soluble and highly emissive. Adv. Funct. Mater. 2012, 22, 1076-1086. [CrossRef] 
58. Sambe, L.; De La Rosa, V.R.; Belal, K.; Stoffelbach, F.; Lyskawa, J.; Delattre, F.; Bria, M.; Cooke, G.; Hoogenboom, R.; Woisel, P. Programmable polymer-based supramolecular temperature sensor with a memory function. Angew. Chem. Int. Ed. 2014, 53, 5044-5048.

59. De la Rosa, V.R.; Hoogenboom, R. Solution polymeric optical temperature sensors with long-term memory function powered by supramolecular chemistry. Chem. Eur. J. 2015, 21, 1302-1311. [CrossRef] [PubMed]

60. Custers, J.P.A.; van Nispen, S.F.G.M.; Can, A.; De la Rosa, V.R.; Maji, S.; Schubert, U.S.; Keurentjes, J.T.F.; Hoogenboom, R. Reversible calcium(II)-ion binding through an apparent $\mathrm{pK}(\mathrm{a})$ shift of thermosensitive block-copolymer micelles. Angew. Chem. Int. Ed. 2015, 54, 14085-14089. [CrossRef] [PubMed]

61. Custers, J.P.A.; Kelemen, P.; Van den Broeke, L.J.P.; Stuart, M.A.C.; Keurentjes, J.T.F. Reversible binding of multivalent ions by surfactant self-assembly. J. Am. Chem. Soc. 2005, 127, 1594-1595. [CrossRef] [PubMed]

62. Mahou, R.; Tran, N.M.; Dufresne, M.; Legallais, C.; Wandrey, C. Encapsulation of Huh-7 cells within alginate-poly(ethylene glycol) hybrid microspheres. J. Mater. Sci. Mater. Med. 2012, 23, 171-179. [CrossRef] [PubMed]

(C) 2016 by the authors. Licensee MDPI, Basel, Switzerland. This article is an open access article distributed under the terms and conditions of the Creative Commons Attribution (CC BY) license (http:/ / creativecommons.org/licenses/by/4.0/). 


\title{
Article \\ A Comprehensive Systematic Study on Thermoresponsive Gels: Beyond the Common Architectures of Linear Terpolymers
}

\author{
Anna P. Constantinou, Hanyi Zhao, Catriona M. McGilvery, Alexandra E. Porter and \\ Theoni K. Georgiou * \\ Department of Materials, Imperial College London, Royal School of Mines, Exhibition Road, \\ London SW7 2AZ, UK; anna.constantinou14@imperial.ac.uk (A.P.C.); hanyi.zhao15@imperial.ac.uk (H.Z.); \\ catriona.mcgilvery@imperial.ac.uk (C.M.M.); a.porter@imperial.ac.uk (A.E.P.) \\ * Correspondence: t.georgiou@imperial.ac.uk; Tel.: +44-20-7594-5177
}

Academic Editor: Alexander Böker

Received: 4 December 2016; Accepted: 16 January 2017; Published: 20 January 2017

\begin{abstract}
In this study, seven thermoresponsive methacrylate terpolymers with the same molar mass (MM) and composition but various architectures were successfully synthesized using group transfer polymerization (GTP). These terpolymers were based on tri(ethylene glycol) methyl ether methacrylate (TEGMA, A unit), n-butyl methacrylate (BuMA, B unit), and 2-(dimethylamino)ethyl methacrylate (DMAEMA, C unit). Along with the more common $\mathrm{ABC}, \mathrm{ACB}, \mathrm{BAC}$, and statistical architectures, three diblock terpolymers were also synthesized and investigated for the first time, namely $(\mathrm{AB}) \mathrm{C}, \mathrm{A}(\mathrm{BC})$, and $\mathrm{B}(\mathrm{AC})$; where the units in the brackets are randomly copolymerized. Two BC diblock copolymers were also synthesized for comparison. Their hydrodynamic diameters and their effective $\mathrm{p} K_{\mathrm{a}} \mathrm{s}$ were determined by dynamic light scattering (DLS) and hydrogen ion titrations, respectively. The self-assembly behavior of the copolymers was also visualized by transmission electron microscopy (TEM). Both dilute and concentrated aqueous copolymer solutions were extensively studied by visual tests and their cloud points $(\mathrm{CP})$ and gel points were determined. It is proven that the aqueous solution properties of the copolymers, with specific interest in their thermoresponsive properties, are influenced by the architecture, with the $\mathrm{ABC}$ and $\mathrm{A}(\mathrm{BC})$ ones to show clear sol-gel transition.
\end{abstract}

Keywords: thermoresponsive polymers; 2-(dimethylamino)ethyl methacrylate; injectable gels; 3-D printing; group transfer polymerization (GTP); terpolymers; complex architectures; well-defined polymers

\section{Introduction}

Thermoresponsive polymers are "smart" polymers which are able to respond to temperature [1-3]. The response of the polymers is indicated by a change of their properties. A special class of thermoresponsive polymers which has become very popular covers polymers that exhibit a Lower Critical Solution Temperature (LCST) behavior. These polymers become insoluble in aqueous media when increasing the temperature. This behavior is explained by the "hydrophobic effect", i.e., the entropy of water becomes the most dominant factor and forces the polymer to precipitate out of solution [2,4]. It is the same phenomenon that in lower temperatures and concentrations forces the polymers to form micelles $[2,4]$. Thermoresponsive polymers with the appropriate structural parameters, and under the appropriate environmental conditions form 3-D networks of physically-interconnected micelles; these 3-D networks are known as thermoresponsive gels [5]. As has been well-demonstrated, the architecture, the molar mass (MM), the composition, and the molar mass distribution (MMD) of the thermoresponsive 
copolymers determine whether, and at which temperature and concentration a gel is formed [1]. In the case of aqueous solutions, these micelles are connected via well-hydrated bridges [5].

Thermoresponsive gels have been widely studied on account of their interesting applications, including tissue engineering as injectable gels [6-8] and more recently in 3-D printing [9-12]. For thermoresponsive polymers to be used as injectable gels a sol-gel transition close to body temperature is required for a minimal invasion administration [13]. On the other hand, for the 3-D printing application the sol-gel transition should occur either close to room or body temperature, depending on the temperature that a stable printed structure is desirable at $[11,14]$. In this case, the thermoresponsive gel should not only possess good mechanical properties in order to maintain the printed structure, but it should also be characterized by good shear-thinning properties [11,14].

For thermoresponsive polymers to be widely applied and industrialized, their synthesis should be easy, time- and cost-effective, reproducible, and scalable. Furthermore, a "living" or "controlled" polymerization technique is required for the synthesis in order to produce copolymers with well-defined structural parameters [15] since it has been well-demonstrated in the literature that these parameters can affect the sol-gel transition [1]. Group transfer polymerization (GTP) is an ideal polymerization method for the synthesis of methacrylate well-defined polymers because: (i) it is a fast polymerization technique (10-15 min per block) [16]; (ii) it produces polymers with narrow MMD (usually below 1.2) and well-defined and controllable composition [16,17]; (iii) it can produce polymers in an industrial scale and (iv) it is cost-effective [17]. GTP is cost-effective for several reasons: it works at higher concentrations than for example anionic polymerization, it is performed at room temperature so there is no need to cool down or heat up the reaction, and there is a $100 \%$ conversion of the monomer to the polymer so sequential one-pot polymerization can be easily achieved for block copolymers without any extra pot(s) or purification steps needed [17]. Therefore, this polymerization technique has been used in the present study and the aim was to investigate how the polymers' architecture affects their polymers' thermoresponsive behavior.

Our group has previously published five research articles on thermoresponsive gels in which several polymeric parameters have been varied [18-22]. In four of the studies, the effects of architecture [18,20], composition [20-22], and MM [21] of triblock and statistical terpolymers on the thermoresponsive properties have been systematically investigated. These terpolymers were based on (i) a poly(ethylene glycol) (PEG) methacrylate unit as the hydrophilic and biocompatible unit (A unit); (ii) an alkyl methacrylate hydrophobic unit (B unit); and (iii) the thermoresponsive and pH-responsive 2-(dimethylamino)ethyl methacrylate (DMAEMA, C unit). The lengths of the alkyl and the PEG side-chains have been also systematically varied by using: (i) ethyl, $n$-butyl, and $n$-hexyl methacrylate (EtMA, BuMA, and HexMA, respectively) [18]; and (ii) di-, penta-, and nona(ethylene glycol) methyl ether methacrylate (DEGMA, PEGMA, and NEGMA, respectively) [22]. It has been demonstrated that the optimum parameters producing polymers with the clearest sol-gel transition and gels of good mechanical properties are: (i) the ABC architecture [18,20]; (ii) MM ranging between $7000-10,000 \mathrm{~g} \cdot \mathrm{mol}^{-1}$ [21]; (iii) intermediate hydrophobic composition of around 30\%-35\% $w / w$ [20-22]; (iv) BuMA as the hydrophobic unit [18]; and (v) a PEG methacrylate unit with ethylene glycol groups between two and five [22].

Given these optimum parameters, new copolymers which are based on the unique combination of these structural characteristics were designed. Therefore, the copolymers are based on BuMA, and DMAEMA as B and C units, respectively, while the A unit was based on tri(ethylene glycol) methyl ether methacrylate (TEGMA). We chose TEGMA instead of the $300 \mathrm{~g} \cdot \mathrm{mol}^{-1} \mathrm{PEGMA}$ that we normally use because based on our latest study we think it will improve the thermoresponsive ability of the terpolymers. The target TEGMA-BuMA-DMAEMA composition was $25 \%-35 \%-40 \%$ $w / w$, while the target $\mathrm{MM}$ was $8200 \mathrm{~g} \cdot \mathrm{mol}^{-1}$. Both the composition and the MM values are within the intermediate range established previously. The aim of this study is not only to use the optimum structural parameters, but also to examine interesting architectures of terpolymers which have not been systematically investigated before. More specifically, apart from the ABC, ACB, BAC, and statistical 
architectures that have been previously studied, in the present study diblock terpolymers have also been synthesized. The three possible combinations that produce different diblock terpolymers are: (i) $(\mathrm{AB}) \mathrm{C}, \mathrm{A}(\mathrm{BC})$, and $\mathrm{B}(\mathrm{AC})$; the units in the brackets have been randomly copolymerized and the resulting block has been polymerized with the third monomer to form the final diblock terpolymer. Two BC diblock bipolymers have also been synthesized to mimic: (i) the BuMA:DMAEMA weight percentages in the terpolymers, and (ii) the hydrophobic:hydrophilic [BuMA:(DMAEMA + TEGMA)] weight percentages in the terpolymers. To the best of our knowledge this is the first time so many different architectures have been synthesized and investigated in terms of their thermoresponsive behavior. Furthermore, it should be noted that the architecture was able to be varied independently i.e., without altering the MM and the composition that is not easy to achieve, but it was because GTP was used.

\section{Experimental}

\subsection{Materials}

TEGMA (monomer, $\mathrm{MM}=232.27 \mathrm{~g} \cdot \mathrm{mol}^{-1}, 94 \%$ ), BuMA (monomer, 99\%), DMAEMA (monomer, $98 \%$ ), activated basic aluminum oxide $\left(\mathrm{Al}_{2} \mathrm{O}_{3} \cdot \mathrm{KOH}\right)$, calcium hydride $\left(\mathrm{CaH}_{2}, \geq 90 \%\right)$, 2,2-diphenyl-1-picrylhydrazyl (DPPH, free-radical inhibitor), potassium metal, sodium metal, tetrahydrofuran (THF, polymerization solvent, HPCL grade, $\geq 99.9 \%$ ), methyl trimethylsilyl dimethylketene acetal (MTS, initiator, 95\%), deuterated chloroform (chloroform-d, 99.8 atom\% D), sodium hydroxide pellets $(\mathrm{NaOH}, 97 \%)$, concentrated hydrochloric acid ( $\mathrm{HCl}$, ACS reagent, 37\%), and hydrochloric acid solution (volumetric, $1 \mathrm{M}$ ) were purchased from Sigma Aldrich Co Ltd., Irvine, UK. Tetrabutylammonium hydroxide ( $40 \%$ in water) and benzoic acid were purchased from Acros Organics-UK distributor Fisher Scientific UK Ltd., Loughborough, UK. Tetrahydrofuran (THF, mobile phase in GPC, GPC grade) and n-hexane (precipitation solvent) were purchased from Fisher Scientific UK Ltd. (Loughborough, UK) and VWR International Ltd. (Lutterworth, UK), respectively. Phosphate buffered saline (PBS, 10x solution) was purchased from Fischer Scientific UK Ltd., Loughborough, UK.

\subsection{Purification of the Starting Materials}

The monomers, TEGMA, BuMA, and DMAEMA, were passed twice through $\mathrm{Al}_{2} \mathrm{O}_{3} \cdot \mathrm{KOH}$ in order to remove the inhibitor (monomethyl ether hydroquinone) and any acidic impurities. DPPH was added in order to prevent free-radical polymerization and the humidity was eliminated by stirring the monomers over $\mathrm{CaH}_{2}$ for $3 \mathrm{~h}$. The monomers were then kept refrigerated until use. The polymerization solvent, THF, was dried by refluxing for 3 days over potassium and sodium metals. The initiator, MTS, and the monomers were distilled under vacuum prior to polymerization. The catalyst, tetrabutylammonium bibenzoate (TBABB), was previously synthesized by tetrabutylammonium hydroxide and benzoic acid, as reported by Dicker et al. [23], and it was dried and kept under vacuum until use. All the glassware was dried overnight at $140{ }^{\circ} \mathrm{C}$ and assembled hot under vacuum. The chemical structures of the monomers, the initiator and the catalyst are shown in Figure 1.

\subsection{Copolymer Synthesis}

All the copolymers were synthesized using a cost-effective and easy to scale-up anionic polymerization technique, specifically group transfer polymerization (GTP), which enables the synthesis of each block in only 10-15 min. The block copolymers were synthesized via sequential addition of the monomers. As an example, the synthesis of polymer 1 , namely TEGMA $-b$-BuMA $20-b-$ DMAEMA $_{21}$ follows: As a first step, TBABB $(\sim 10 \mathrm{mg})$ was added in a $250 \mathrm{~mL}$ round-bottom flask. This flask was sealed with a rubber septum and purged with argon, followed by the addition of $60 \mathrm{~mL}$ freshly-distilled THF using a syringe. MTS $(0.37 \mathrm{~mL}, 0.32 \mathrm{~g}, 1.8 \mathrm{mmol})$ was then syringed into the flask. The addition of the TEGMA monomer $(3.6 \mathrm{~mL}, 3.7 \mathrm{~g}, 15.9 \mathrm{mmol})$ followed and the exothermic reaction was monitored; the temperature was increased from 24.2 to $28.3^{\circ} \mathrm{C}$. As soon as the reaction 
was complete, two samples of $0.1 \mathrm{~mL}$ were obtained for GPC and ${ }^{1} \mathrm{H}-\mathrm{NMR}$ analysis. Following this, BuMA (5.8 mL, $5.2 \mathrm{~g}, 36.3 \mathrm{mmol})$ was added, thus the temperature was increased from 25.2 to $28.9^{\circ} \mathrm{C}$. Two samples of $0.1 \mathrm{~mL}$ were obtained in order to analyze them by GPC and ${ }^{1} \mathrm{H}-\mathrm{NMR}$. As a last step, $6.3 \mathrm{~mL}$ of DMAEMA $(5.9 \mathrm{~g}, 37.5 \mathrm{mmol})$ was added and an exotherm from 27.6 to $31.8^{\circ} \mathrm{C}$ was observed. GPC and ${ }^{1} \mathrm{H}-\mathrm{NMR}$ samples $(0.1 \mathrm{~mL}$ each) were extracted for analysis. The polymers were precipitated in cool $n$-hexane and dried in a vacuum oven at room temperature. In this study, seven terpolymers of the same composition and target MM, but different architectures were synthesized. This was achieved by keeping the amount of monomers the same, while the order of each monomer addition was varied. More specifically, concerning the diblock terpolymers, the statistical bipolymer was synthesized via simultaneous addition of the two monomers. The statistical terpolymer was synthesized by adding all the monomers prior to the addition of the MTS. Two diblock copolymers based only on BuMA and DMAEMA with different compositions were also synthesized by adding different amounts of the monomers.<smiles>[Z]C(C)(C)OCCOCCOC(=O)C(=C)C</smiles>

Tri(ethylene glycol) methyl ether methacrylate

(TEGMA)

Monomer

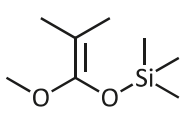

Methyl trimethylsilyl dimethylketene acetal (MTS) Initiator<smiles>C=C(C)C(=O)OCCCC</smiles>

n-Butyl methacrylate (BuMA)

Monomer

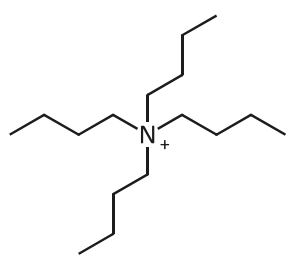

Tetrabutylammonium bibenzoate (TBABB) Catalyst<smiles>COC(=O)c1ccccc1</smiles><smiles>C=C(C)C(=O)OCCN(C)C</smiles>

2-(Dimethylamino) ethyl methacrylate

(DMAEMA)

Monomer

$\sqrt{2}$

Figure 1. Chemical structures of the monomers, the initiator, and the catalyst.

\subsection{Characterization in Organic Solvents}

The MM, the molar mass distribution (MMD), and the composition of all the copolymers and their linear precursors were determined in organic solvents.

\subsubsection{Gel Permeation Chromatography (GPC)}

The final copolymers and their precursors were characterized in terms of their MM and MMD by GPC. For this, an Agilent SECurity GPC system, with a Polymer Standard Service (PSS) SDV analytical linear M column (SDA083005LIM) was used (Agilent technologies UK Ltd., Shropshire, UK). This system is equipped with a "1260 Iso" isocratic pump and an Agilent 1260 refractive index (RI) detector. As a mobile phase, THF with $5 \%$ vol triethylamine was used, which was pumped with a flow rate of $1 \mathrm{~mL} \cdot \mathrm{min}^{-1}$. The calibration curve was plotted by running six different linear poly(methyl methacrylate) (PMMA) standard samples with MM equal to 2000, 4000, 8000, 20,000, 50,000, and $100,000 \mathrm{~g} \cdot \mathrm{mol}^{-1}$, purchased from Fluka, Sigma Aldrich Co Ltd., Irvine, UK. 


\subsubsection{Proton Nuclear Magnetic Resonance Spectroscopy $\left({ }^{1} \mathrm{H}-\mathrm{NMR}\right)$}

The final copolymers and their precursors were characterized in terms of their composition by ${ }^{1} \mathrm{H}-\mathrm{NMR}$ using a $400 \mathrm{MHz}$ Avance Bruker NMR spectrometer (Bruker UK Ltd., Coventry, UK). The ${ }^{1} \mathrm{H}$-NMR spectra were obtained using $\mathrm{CDCl}_{3}$ as the deuterated solvent.

\subsection{Characterization in Aqueous Solution}

The effective dissociation constants $\left(\mathrm{p} K_{\mathrm{a}}\right)$, the hydrodynamic diameters $\left(d_{\mathrm{h}}\right)$, the cloud points $(\mathrm{CP})$, and the thermal response of copolymers in aqueous solutions were determined.

\subsubsection{Hydrogen Ion Titrations}

Hydrogen ion titrations of $1 \% w / w$ aqueous polymer solutions were performed by using a HI98103 pH-checker from Hanna instruments Ltd., Leighton Buzzard, UK. The solutions were titrated from $\mathrm{pH} 2$ to $\mathrm{pH} 12$ using a $0.25 \mathrm{M} \mathrm{NaOH}$ solution. The start- and end-point of the titration of the DMAEMA units were determined by plotting the first derivative of the titration curve and the effective $\mathrm{p} K_{\mathrm{a}}$ was determined as the $\mathrm{pH}$ of the solution at which the amino groups were protonated by $50 \%$.

\subsubsection{Dynamic Light Scattering (DLS)}

DLS measurements of $1 \% w / w$ aqueous polymer solutions ( $\mathrm{pH}$ adjusted to 6 and 7) were conducted by using a Zetasizer Nano ZSP instrument from Malvern Instruments Ltd., Malvern, UK. The polymer solutions were filtered using nylon $0.45 \mu \mathrm{m}$ PTFE syringe filters in order to remove any dust and bigger aggregates. After filtering, the solutions were allowed to settle in order to ensure complete bubble removal. Three DLS experiments were performed per sample at room temperature and the scattered light was collected at a backscatter angle of $173^{\circ}$. The $d_{\mathrm{h}} \mathrm{s}$ reported are the mean values determined as the diameters corresponding to the peak of maximum intensity.

These experimental $d_{\mathrm{h}} \mathrm{s}$ were compared to the theoretical ones. Four different theoretical models were applied depending on the polymer architecture. Concerning the block copolymers, formation of spherical micelles was assumed and the calculations were based on the projected length of the methacrylate unit, equal to $0.254 \mathrm{~nm}$, and the experimental degree of polymerization (DP). (1) When the $\mathrm{ABC}$ and $\mathrm{BC}$ architectures are concerned, the theoretical diameter was calculated as follows: $d=\left(\mathrm{DP}_{\text {BuMA }}+2 \times \mathrm{DP}_{\text {DMAEMA }}\right) \times 0.254 \mathrm{~nm}$; (2) When the hydrophobic BuMA unit forms a distinct block at the end of the polymer chain, i.e., $\mathrm{ACB}, \mathrm{BAC}$, and $\mathrm{B}(\mathrm{AC})$, the theoretical values were calculated using the following equation: $d=\left[\mathrm{DP}_{\mathrm{BuMA}}+2 \times\left(\mathrm{DP}_{\mathrm{DMAEMA}}+\mathrm{DP}_{\mathrm{TEGMA}}\right)\right] \times 0.254 \mathrm{~nm}$; (3) When the hydrophobic BuMA unit is randomly copolymerized with either of the hydrophilic monomer (TEGMA or DMAEMA), while the other hydrophilic monomer is in a block structure, i.e., $(A B) C$ and $A(B C)$, the following formulae were used: $d=\left(\mathrm{DP}_{\text {BuMA }}+\mathrm{DP}_{\text {TEGMA }}+2 \times \mathrm{DP}_{\mathrm{DMAEMA}}\right) \times 0.254 \mathrm{~nm}$ and $d=\left(\mathrm{DP}_{\mathrm{BuMA}}+\mathrm{DP}_{\mathrm{DMAEMA}}+2 \times \mathrm{DP}_{\mathrm{TEGMA}}\right) \times 0.254 \mathrm{~nm}$; (4) When a random copolymer is concerned, random coil is assumed to be formed, the diameter of which is calculated according to the following equation: $\left\langle d_{\mathrm{g}}{ }^{2}\right\rangle^{1 / 2}=2 \times\left[2 \times 2.20 \times\left(\mathrm{DP}_{\mathrm{TEGMA}}+\mathrm{DP}_{\mathrm{BuMA}}+\mathrm{DP}_{\mathrm{DMAEMA}}\right) / 3\right]^{1 / 2} \times 0.154 \mathrm{~nm}$. For these calculations, the experimental DPs were used, as calculated from GPC and ${ }^{1} \mathrm{H}-\mathrm{NMR}$ results.

\subsubsection{Transmission Electron Microscopy (TEM)}

The TEM images were recorded using an FEI Titan 80-300 transmission electron microscope (TEM) (FEI (part of Thermo Fisher Scientific, Hillsboro, OR, USA), equipped with an image corrector. The instrument was operated at $80 \mathrm{kV}$ to enhance contrast for bright field TEM, and an objective aperture of $70 \mu \mathrm{m}$ was used. $1 \% w / w$ aqueous copolymer solutions ( $\mathrm{pH}$ adjusted at 6 ) was used for the preparation of the TEM samples. The TEM samples were prepared by pipetting $3.5 \mu \mathrm{L}$ of solution onto holey-carbon grids. After two minutes any excess of solvent was removed using filter paper. To increase contrast in the TEM the samples were then negatively stained by adding $30 \mu \mathrm{L}$ of $1 \% w / v$ 
uranyl acetate solution to the grid, while the TEM grids were held at an angle of $45^{\circ}$. Any remaining excess solution was removed with filter paper, and the grids were left to dry.

\subsubsection{Visual Tests}

The visual tests were performed using an IKA RCT stirrer hotplate (IKA ${ }^{\circledR}$ England Ltd., Oxford, UK), equipped with an IKA ETS-D5 temperature controller, and a continuously-stirred water-bath. For the determination of the CPs, $1 \% w / w$ aqueous copolymer solutions were used, whereas for the construction of the phase diagrams, 1\%, 2\%, 5\%, 10\%, 15\%,20\%,25\%, and 30\% w/w copolymer solutions in PBS, were tested. In both cases, the DMAEMA units were protonated by $10 \%$ to enhance solubility. The vials were suspended in a water-bath and a thermal response was visually inspected every one degree from 20 to $80^{\circ} \mathrm{C}$. The $\mathrm{CP}$ was determined as the temperature at which the solution turned cloudy, while the gel point was determined as the temperature at which a stable gel was formed, which did not flow upon tube inversion.

\section{Results and Discussion}

In this study, the synthesis of seven terpolymers was achieved using GTP and the architecture was systematically varied, while the TEGMA-BuMA-DMAEMA composition and the MM were kept constant at $25 \%-35 \%-40 \% w / w$ and $8200 \mathrm{~g} \cdot \mathrm{mol}^{-1}$, respectively. More specifically, three triblock terpolymers $(\mathrm{ABC}, \mathrm{CAB}$, and $\mathrm{BAC})$, three diblock terpolymers $((\mathrm{AB}) \mathrm{C}, \mathrm{A}(\mathrm{BC})$, and $\mathrm{B}(\mathrm{AC}))$ and one statistical copolymer were synthesized in order to investigate the effect of architecture on the thermoresponsive behavior. For comparison, two BC diblock copolymers were also synthesized, with the same MM but with the BuMA-DMAEMA weight percentages mimicking the BuMA-DMAEMA and the hydrophobic-hydrophilic weight ratio in the terpolymers. The structures of the copolymers are schematically shown in Figure 2. The TEGMA, BuMA, and DMAEMA units are represented by blue, orange, and green spheres, respectively.
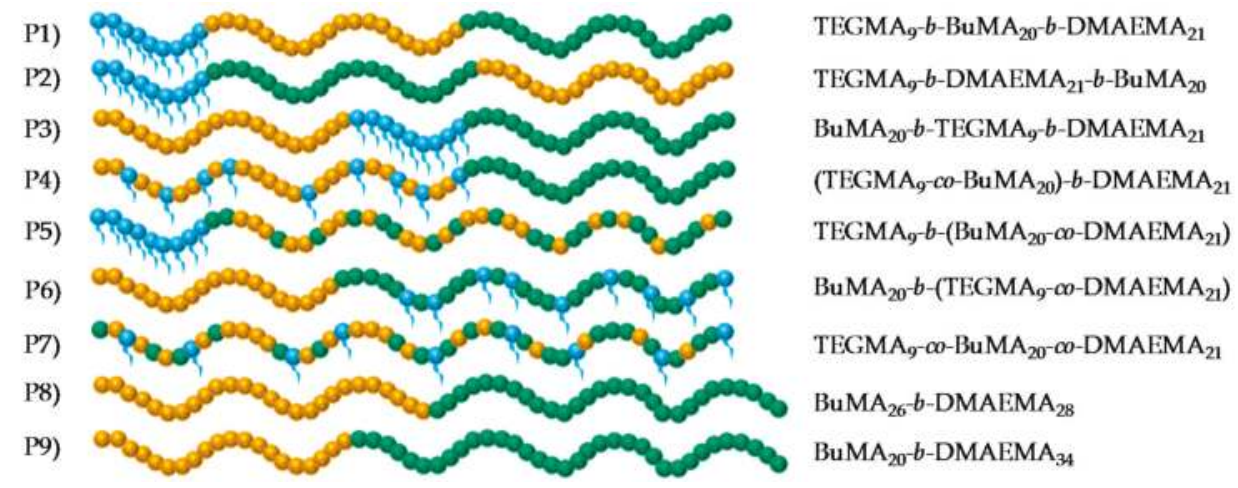

Figure 2. Schematic showing the structures of all the copolymers studied. Blue, orange, and green colors represent the TEGMA, BuMA, and DMAEMA units, respectively.

\subsection{Structural Properties}

Table 1 summarizes the structural properties of all the copolymers and their linear precursors. Specifically, the theoretical MMs, and the experimental MMs and MMDs as resulted from GPC analysis are shown; the latter are shown as dispersity indices $(\bigoplus)$. The theoretical compositions and the experimental ones, as resulted by ${ }^{1} \mathrm{H}-\mathrm{NMR}$ analysis, are also listed in Table 1. 
Table 1. Theoretical and experimental molar masses (MMs) and compositions, and molar mass distributions (MMDs) of the copolymers and their precursors.

\begin{tabular}{|c|c|c|c|c|c|c|}
\hline \multirow[t]{2}{*}{ Polymer No. } & \multirow[t]{2}{*}{ Theoretical polymer structure ${ }^{a}$} & \multicolumn{2}{|c|}{$\begin{array}{c}\% w / w \\
\text { TEGMA-BuMA-DMAEMA }\end{array}$} & \multirow{2}{*}{$\begin{array}{l}\text { MM Theor. } \\
\left(\mathrm{g} \cdot \mathrm{mol}^{-1}\right)\end{array}$} & \multirow[t]{2}{*}{$\begin{array}{c}M_{\mathrm{n}}{ }^{\mathrm{c}} \\
\left(\mathrm{g} \cdot \mathrm{mol}^{-1}\right)\end{array}$} & \multirow[t]{2}{*}{$\bigoplus^{\mathrm{c}}$} \\
\hline & & Theoretical & ${ }^{1} \mathrm{H}-\mathrm{NMR}$ & & & \\
\hline \multirow[t]{3}{*}{1} & TEGMA $_{9}$ & $100-00-00$ & $100-00-00$ & 2125 & 3020 & 1.12 \\
\hline & TEGMA $_{9}-b$-BuMA 20 & $42-58-00$ & $42-58-00$ & 4960 & 6600 & 1.12 \\
\hline & TEGMA $_{9}-b$-BuMA $20-b$-DMAEMA 21 & $25-35-40$ & $25-35-40$ & 8200 & 10,700 & 1.17 \\
\hline \multirow[t]{3}{*}{2} & TEGMA $_{9}$ & $100-00-00$ & $100-00-00$ & 2125 & 3030 & 1.11 \\
\hline & TEGMA $_{9}-b$-DMAEMA 21 & $38-00-62$ & $38-00-62$ & 5365 & 7650 & 1.11 \\
\hline & TEGMA $_{9}-b$-DMAEMA $21-b-$ BuMA $_{20}$ & $25-35-40$ & $26-34-40$ & 8200 & 10,800 & 1.16 \\
\hline \multirow[t]{3}{*}{3} & $\mathrm{BuMA}_{20}$ & $00-100-00$ & $00-100-00$ & 2935 & 2980 & 1.12 \\
\hline & BuMA $_{20}-b$-TEGMA 9 & $42-58-00$ & $41-59-00$ & 4960 & 6700 & 1.08 \\
\hline & BuMA $_{20}-b$-TEGMA $-b$-DMAEMA 21 & $25-35-40$ & $26-35-39$ & 8200 & 10,100 & 1.13 \\
\hline \multirow[t]{2}{*}{4} & $\mathrm{TEGMA}_{9}-\mathrm{co}-\mathrm{BuMA}_{20}$ & $42-58-00$ & $42-58-00$ & 4960 & 5940 & 1.09 \\
\hline & $\left(\right.$ TEGMA $\left._{9}-c o-\mathrm{BuMA}_{20}\right)-b$-DMAEMA 21 & $25-35-40$ & $26-35-39$ & 8200 & 8780 & 1.10 \\
\hline \multirow[t]{2}{*}{5} & TEGMA9 $_{9}$ & $100-00-00$ & $100-00-00$ & 2125 & 2810 & 1.12 \\
\hline & TEGMA $_{9}-b-\left(\mathrm{BuMA}_{20}-\mathrm{co}-\mathrm{DMAEMA}_{21}\right)$ & $25-35-40$ & $26-34-40$ & 8200 & 9950 & 1.14 \\
\hline \multirow[t]{2}{*}{6} & $\mathrm{BuMA}_{20}$ & $00-100-00$ & $00-100-00$ & 2935 & 4180 & 1.10 \\
\hline & BuMA $_{20}-b-\left(\right.$TEGMA$\left._{9}-\mathrm{co}-\mathrm{DMAEMA}_{21}\right)$ & $25-35-40$ & $26-37-37$ & 8200 & 8580 & 1.13 \\
\hline 7 & $\mathrm{TEGMA}_{9}-\mathrm{co}-\mathrm{BuMA}_{20}$-co-DMAEMA 21 & $25-35-40$ & $26-35-39$ & 8200 & 8760 & 1.10 \\
\hline \multirow[t]{2}{*}{8} & $\mathrm{BuMA}_{26}$ & $00-100-00$ & $00-100-00$ & 3826 & 6240 & 1.07 \\
\hline & BuMA $_{26}-b$-DMAEMA 28 & $00-46-54$ & $00-47-53$ & 8200 & 11,000 & 1.11 \\
\hline \multirow[t]{2}{*}{9} & $\mathrm{BuMA}_{20}$ & $00-100-00$ & $00-100-00$ & 2935 & 4040 & 1.10 \\
\hline & BuMA $_{20}-b$-DMAEMA 34 & $00-35-65$ & $00-37-63$ & 8200 & 9380 & 1.10 \\
\hline
\end{tabular}

a TEGMA, BuMA, and DMAEMA stand for tri(ethylene glycol) methyl ether methacrylate, $n$-butyl methacrylate, and 2-(dimethylamino) ethyl methacrylate, respectively; ${ }^{\mathrm{b}}$ The theoretical MM of the polymer was calculated as follows: $\left(\mathrm{DP}_{\text {TEGMA }} \times \mathrm{MM}_{\text {TEGMA }}\right)+\left(\mathrm{DP}_{\text {BuMA }} \times \mathrm{MM}_{\text {BuMA }}\right)+\left(\mathrm{DP}_{\text {DMAEMA }}+\mathrm{MM}_{\text {DMAEMA }}\right)+100 \mathrm{~g} \cdot \mathrm{mol}^{-1}$; $\mathrm{MM}$ and DP stand for molar mass and degree of polymerization, respectively. The value of $100 \mathrm{~g} \cdot \mathrm{mol}^{-1}$ is the part of the MTS initiator which stays on the polymer chain; ${ }^{\mathrm{c}}$ The $M_{\mathrm{n}}$ and $Ð$ were determined by gel permeation chromatography (GPC). The calibration curve was plotted using six linear poly (methyl methacrylate) (pMMA) standard samples of MM equal to 2000, 4000, 8000, 20,000, 50,000, and $100,000 \mathrm{~g} \cdot \mathrm{mol}^{-1}$.

\subsubsection{Molar Masses and Molar Mass Distributions}

As can be seen in Table 1 , the number-average MM $\left(M_{n}\right)$ values of the final copolymers vary between 8550 and $11,000 \mathrm{~g} \cdot \mathrm{mol}^{-1}$, which are within the desirable range for obtaining a clear sol-gel transition. When the experimental $M_{n}$ values of the final copolymers and their precursors are compared to the theoretical ones, they are slightly higher. This is ascribed to (i) the calibration curve being based on PMMA standard samples, and (ii) the partial deactivation of the initiator, MTS, i.e., an amount of the initiator molecules is terminated by the presence of humidity or any other protic impurities. This is consistent with other studies on polymers synthesized via GTP [18-22,24].

In Table 1 , the $Ð$ values are also given, which are satisfactorily close to the ideal value of unity (varying between 1.07 and 1.17). This confirms the successful "living" GTP, similar to previously reported studies [18-22,24]. Concerning the $Ð$ values of the final copolymers, it can be generally observed that lower $Ð$ values were obtained for the $\mathrm{BC}$ diblock copolymers; these copolymers are the $\mathrm{BuMA}_{26}-b$-DMAEMA 28 (Polymer 8) and $\mathrm{BuMA}_{20}-b$-DMAEMA 34 (Polymer 9). This can be attributed to the absence of the TEGMA units, which are macromonomers with average MM, thus meaning that they have wider MMD compared to the BuMA and DMAEMA monomers with well-defined structure and MM. This is supported by other GTP studies in which a PEGMA-based macromonomer has been incorporated into the polymer structure [22,25]. Also, lower $Đ$ values are observed in the case of the statistical copolymer TEGMA 9 -co-BuMA $20-c o-D_{M A E M A}$ (Polymer 7) and the $\left(\mathrm{TEGMA}_{9}-\mathrm{co}-\mathrm{BuMA}_{20}\right)-b$-DMAEMA 21 (Polymer 4). To produce the statistical copolymer, Polymer 7 , all monomers were added to the flask prior to the initiator and the flask was cooled down in a room temperature water bath to avoid the flask overheating because the monomers were not added dropwise. This resulted in a more controlled polymerization and a synthesized polymer with 
a narrower MMD was produced unlike previous studies where the flask was not cooled down and the statistical copolymer had a broader $Ð[19,22]$. Polymer 4 (TEGMA $\left.-c o-B_{1} A_{20}\right)-b-D_{M A E M A} 21$ also had a very narrow MMD probably due to the first block being a statistical block that contained TEGMA and its simultaneous polymerization with BuMA assisted a controlled polymerization.

Figure 3 shows the GPC traces of the TEGMA $9-b-\mathrm{BuMA}_{20}-b-\mathrm{DMAEMA}_{21}$ (Polymer 1 ) before and after precipitation, shown in green solid and dashed line, respectively, and its precursors (TEGMA, and TEGMA $_{9}-b-\mathrm{BuMA}_{20}$, colored in blue and orange, respectively). Figure 3 confirms the successful sequential GTP since the peak appears at higher MM as the polymerization progresses (from the homopolymer to the diblock to the triblock). By precipitating, the lower polymer chains are lost in the precipitation solvent, thus the MM shifts at higher MM. The low intensity shoulder at lower MM corresponds to the TEGMA homopolymer, which slightly prevented further polymerization. Also, it is worth-noting that no peak related to the monomers was observed, thus indicating complete consumption and $100 \%$ conversion of the monomers to the polymer. These observations are the same for all the copolymers, the GPC traces of which can be found in Figure S1 in the Supplementary Materials.

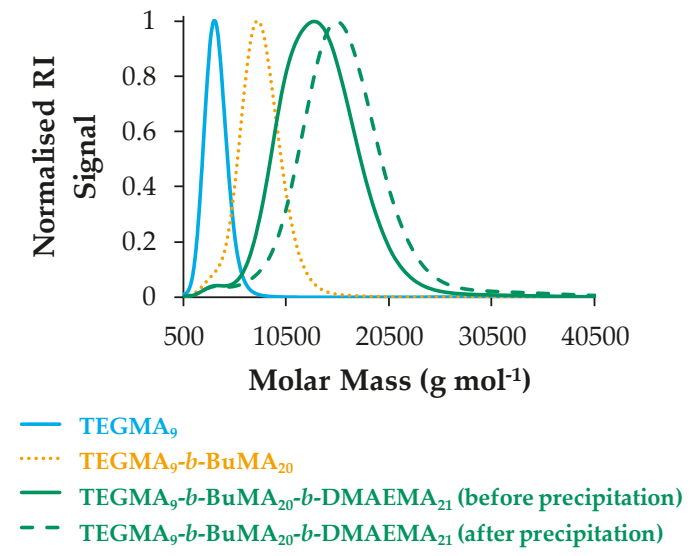

Figure 3. Gel permeation chromatography (GPC) traces of Polymer 1 (TEGMA9- $b$-BuMA $20-b$ DMAEMA $_{21}$ ) and its precursors. The TEGMA homopolymer (TEGMA9), the diblock (TEGMA $-b$ BuMA $_{20}$ ), and the triblock copolymer before precipitation are shown in blue solid, orange dotted and green solid lines, respectively. The triblock copolymer after precipitation is indicated by green dashed line.

\subsubsection{Compositions}

The ${ }^{1} \mathrm{H}-\mathrm{NMR}$ results show good agreement between the theoretical and experimental compositions of the final copolymers and their precursors; these values are listed in Table 1. The experimental compositions were calculated by using the integral of three distinctive peaks belonging to the three different repeated units (see Figure S2 in the Supplementary Materials, which shows the NMR spectra of Polymer 1 and its precursors). The distinctive peak of the TEGMA unit is the one at $3.35 \mathrm{ppm}$ and belongs to the three methoxy protons, whereas the one of BuMA appears at $3.9 \mathrm{ppm}$ and belongs to the two methylene protons of the side-chain closest to the ester. The peak at $2.25 \mathrm{ppm}$ is the one used for DMAEMA and it corresponds to the six methyl protons next to the nitrogen. 


\subsection{Aqueous Solution Properties}

\subsubsection{Hydrodynamic Diameters}

Table 2 lists the theoretical hydrodynamic diameters, calculated by assuming spherical micelle and random coil formation by the block and statistical copolymers, respectively. The experimental hydrodynamic diameters of the copolymers in aqueous solutions at both $\mathrm{pH} 6$ and 7 , as obtained by DLS, are also summarized in Table 2.

Concerning the theoretical calculations, the amphiphilic block copolymers are assumed to form spherical micelles with the BuMA-based block (either as homopolymer or random copolymer with one of the hydrophilic units) to form the core of the micelle. The structures of the spherical micelles, as well as the random coil configuration assumed to be adopted by the block and the statistical copolymers, respectively, are illustrated schematically in Figure 4. The TEGMA, BuMA, and DMAEMA units are represented by blue, orange, and green spheres, respectively.

Table 2. Theoretical and experimental hydrodynamic diameters, effective $\mathrm{p} K_{\mathrm{a}} \mathrm{s}$ and cloud points of $1 \% w / w$ aqueous copolymer solutions.

\begin{tabular}{|c|c|c|c|c|c|c|c|}
\hline \multirow{3}{*}{$\begin{array}{c}\text { Polymer } \\
\text { No. }\end{array}$} & \multirow{3}{*}{ Theoretical polymer structure ${ }^{a}$} & \multicolumn{3}{|c|}{ Hydrodynamic diameter (nm) } & \multirow{3}{*}{$\begin{array}{c}\text { Effective } \\
\mathrm{p} K_{\mathrm{a}} \mathrm{s} \pm 0.1\end{array}$} & \multicolumn{2}{|c|}{ Cloud Points $\pm 2{ }^{\circ} \mathrm{C}$} \\
\hline & & \multirow{2}{*}{ Theoretical $^{b}$} & \multicolumn{2}{|c|}{ Experimental \pm 0.5} & & \multirow{2}{*}{$0 \% \mathrm{H}^{+}$} & \multirow{2}{*}{$10 \% \mathrm{H}^{+}$} \\
\hline & & & $\mathrm{pH}=7$ & $\mathrm{pH}=6$ & & & \\
\hline 1 & TEGMA $_{9}-b-$ BuMA $_{20}-b$-DMAEMA 21 & $20.5^{c}$ & 8.7 & 6.5 & 6.7 & 29 & No CP \\
\hline 2 & TEGMA $_{9}-b$-DMAEMA $21-b$-BuMA 20 & $26.6^{d}$ & 32.7 & 32.7 & 6.8 & NA & No CP \\
\hline 3 & BuMA $_{20}-b$-TEGMA $-b$-DMAEMA 21 & $24.8^{\mathrm{d}}$ & 18.2 & 13.5 & 6.9 & NA & No CP \\
\hline 4 & $\left(\right.$ TEGMA $\left._{9}-c o-\mathrm{BuMA}_{20}\right)-b$-DMAEMA 21 & $19.1^{\mathrm{e}}$ & 21.0 & 24.4 & 6.9 & No CP & No CP \\
\hline 5 & TEGMA $_{9}-b-\left(\mathrm{BuMA}_{20}-\mathrm{co}-\mathrm{DMAEMA}_{21}\right)$ & $18.1^{\mathrm{f}}$ & 25.5 & 21.0 & 6.2 & 32 & No $C P$ \\
\hline 6 & BuMA $_{20}-b-\left(\right.$TEGMA$_{9}-\mathrm{co}$-DMAEMA 21$)$ & $20.8^{\mathrm{d}}$ & 28.2 & 18.2 & 6.7 & NA & No CP \\
\hline 7 & TEGMA $_{9}-c o-\mathrm{BuMA}_{20}-c o-\mathrm{DMAEMA}_{21}$ & $2.7 \mathrm{~g}$ & NA & 15.7 & 6.1 & NA & NA \\
\hline 8 & BuMA $_{26}-b$-DMAEMA 28 & $28.1^{c}$ & 32.7 & 15.7 & 6.5 & NA & No CP \\
\hline 9 & BuMA $_{20}-b$-DMAEMA 34 & $25.3^{c}$ & 21.0 & 15.7 & 6.8 & NA & No CP \\
\hline
\end{tabular}

a TEGMA, BuMA, and DMAEMA stand for tri(ethylene glycol) methyl ether methacrylate, $n$-butyl methacrylate, and 2-(dimethylamino) ethyl methacrylate, respectively; ${ }^{\mathrm{b}}$ The theoretical hydrodynamic diameters $\left(d_{\mathrm{h}}\right)$ were calculated by taking into account the experimental degrees of polymerization (DP) as resulted from GPC and ${ }^{1} \mathrm{H}-\mathrm{NMR}$ analysis; ${ }^{\mathrm{C}}$ The theoretical values of the $\mathrm{ABC}$ triblock terpolymer and the $\mathrm{BC}$ diblock bipolymers were calculated based on the following equation: $d_{\mathrm{h}}=\left(\mathrm{DP}_{\mathrm{BuMA}}+2 \times \mathrm{DP}_{\mathrm{DMAEMA}}\right) \times 0.254 \mathrm{~nm}$; ${ }^{\mathrm{d}}$ For the calculation of the theoretical hydrodynamic diameters of the $\mathrm{ACB}$ and $\mathrm{BAC}$ triblock and the $\mathrm{B}(\mathrm{AC})$ diblock terpolymers, the following equation was used: $d_{\mathrm{h}}=\left[\mathrm{DP}_{\text {BuMA }}+2 \times\left(\mathrm{DP}_{\mathrm{DMAEMA}}+\mathrm{DP}_{\mathrm{TEGMA}}\right)\right] \times 0.254 \mathrm{~nm}$; ${ }^{\mathrm{e}}$ The following formula was used in the case of the $(\mathrm{AB}) \mathrm{C}$ diblock terpolymer: $d_{\mathrm{h}}=\left(\mathrm{DP}_{\text {BuMA }}+\mathrm{DP}_{\text {TEGMA }}+2 \times \mathrm{DP}_{\text {DMAEMA }}\right) \times 0.254 \mathrm{~nm}$; ${ }^{\mathrm{f}}$ In the case of the $\mathrm{A}(\mathrm{BC})$ diblock terpolymer, the theoretical value was calculated according to the following equation: $d_{\mathrm{h}}=\left(\mathrm{DP}_{\mathrm{BuMA}}+\mathrm{DP}_{\mathrm{DMAEMA}}+2 \times \mathrm{DP}_{\mathrm{TEGMA}}\right) \times 0.254 \mathrm{~nm} ;{ }^{\mathrm{g}}$ A random coil configuration was assumed to be adopted by the statistical copolymer and therefore the equation for a random coil was used: $\left\langle d_{\mathrm{g}}{ }^{2}\right\rangle^{1 / 2}=2 \times$ $\left[2 \times 2.20 \times\left(\mathrm{DP}_{\text {TEGMA }}+\mathrm{DP}_{\text {BuMA }}+\mathrm{DP}_{\text {DMAEMA }}\right) / 3\right]^{1 / 2} \times 0.154 \mathrm{~nm} ; \mathrm{NA}$ Testing these copolymer solutions was not feasible because the copolymers were insoluble at this $\mathrm{pH}$.

As can be seen in Table 2, the experimental hydrodynamic diameters of the block terpolymers depend on the polymer architecture. At both $\mathrm{pH}$ values, the experimental sizes of Polymer 1, and 3, with $\mathrm{ABC}$ and $\mathrm{BAC}$ architecture, respectively, are smaller than the theoretical ones, as expected and observed before [20-22]. This is also valid for the solution of Polymer 6 (with B(AC) architecture) at $\mathrm{pH} 6$, at which the DMAEMA units are more than $50 \%$ protonated (as it is discussed in the section on effective $\mathrm{p} K_{\mathrm{a}}$ ). This trend can be attributed to the theoretical model assuming that (i) the methacrylate backbone is fully extended and (ii) the BuMA-based block fully overlaps. In reality, this is not the case since (i) the hydrophobic block is in the collapsed state on account of its incompatibility with the aqueous solvent and (ii) the polymer chains might overlap in a bigger extent, thus reducing the size of the micelles. The opposite effect is observed in the rest of the cases on block terpolymers, i.e., Polymer 2, 4, 5 with $\mathrm{ACB},(\mathrm{AB}) \mathrm{C}$, and $\mathrm{A}(\mathrm{BC})$ architecture at both $\mathrm{pH}$ values and Polymer $6[\mathrm{~B}(\mathrm{AC})]$ at $\mathrm{pH} 7$. This can be attributed to several factors including (i) the $\mathrm{DP}_{\mathrm{BuMA}}$ being higher than in the other studies, or the difference in hydrophilicity between the repeated units being less pronounced, i.e., at $\mathrm{pH} 7$, thus enhancing the hydrophobic interactions and aggregation, (ii) the core-forming block overlapping in a 
lesser extent, and (iii) the theoretical model of Polymer 4 and 5 assuming that the hydrophilic either TEGMA or DMAEMA, which are randomly copolymerized with BuMA, respectively, take part in the core formation, which might not be the case.

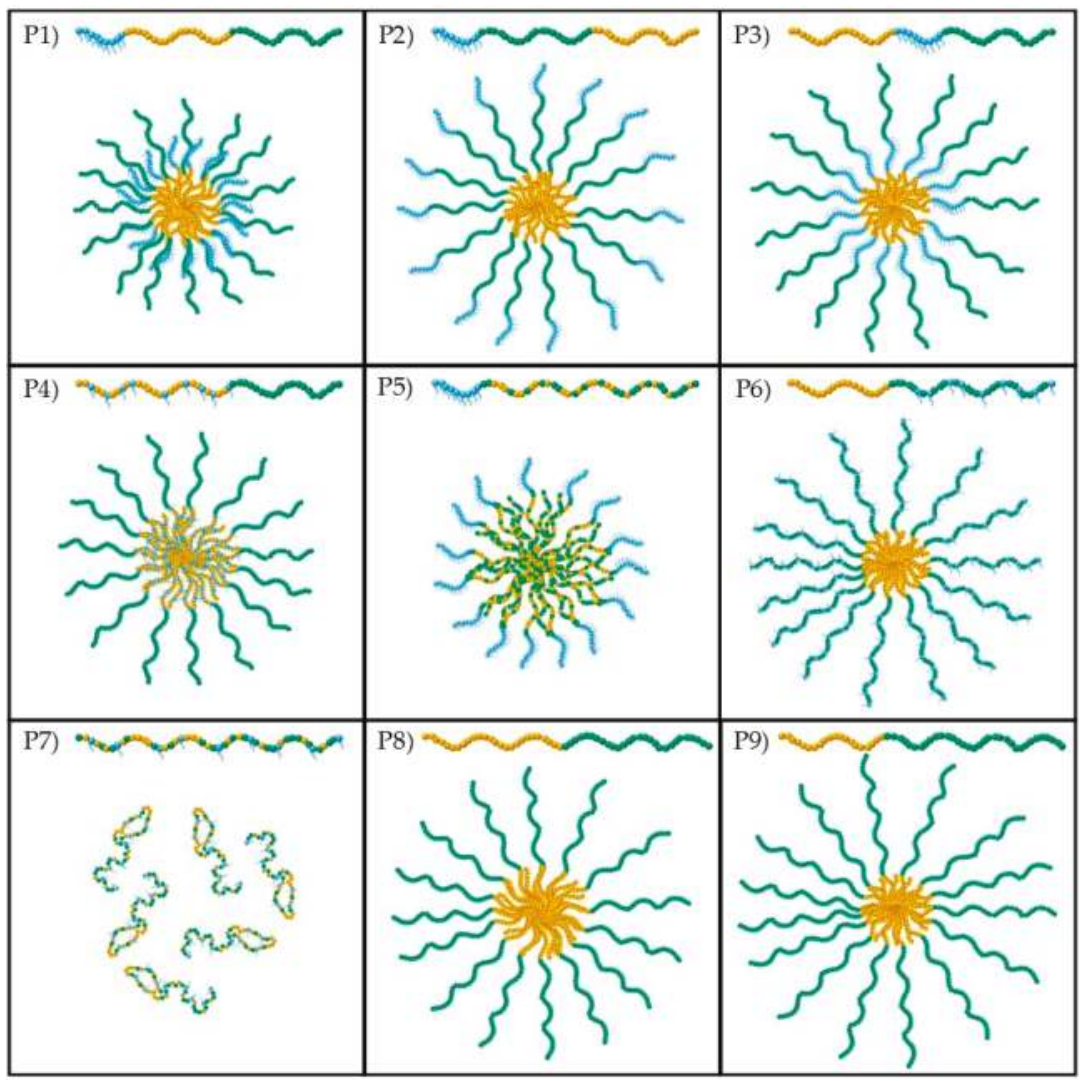

Figure 4. Schematic showing the structures of the spherical micelles adopted by the block copolymers (Polymers 1-6, 8, and 9). The random coil formed by the statistical copolymer (Polymer 7) is also shown. The TEGMA, BuMA, and DMAEMA units are colored in blue, orange, and green, respectively.

Concerning the diblock bipolymers, Polymer 9, which is the most hydrophilic, shows the expected trend at both $\mathrm{pH}$ values, i.e., the experimental values are smaller than the theoretical values. Concerning Polymer 8, the expected trend is observed at $\mathrm{pH} 6$ at which the difference in the hydrophobicity of BuMA and DMAEMA is more pronounced. However, at $\mathrm{pH} 7$, the opposite trend is observed which can be attributed to the effect of hydrophobicity. This confirms the explanation given before about the hydrophobicity enhancing the aggregation to some extent.

The experimental value of the statistical copolymer is almost six times higher than the theoretical one corresponding to random coil configuration. This is consistent with other studies, in which bigger structures were detected by DLS $[18,22]$. This can be ascribed to (i) the presence of the lengthy side chains which might favor aggregation [26], and (ii) the good compatibility of TEGMA and DMAEMA with water, thus enhancing the interactions with water and favoring the extension of the polymer chain [18]. In addition, the experimental value of $15.7 \mathrm{~nm}$ is closer to the value of $13.5 \mathrm{~nm}$ which corresponds to the length of one fully extended polymer chain $\left[\left(\mathrm{DP}_{\mathrm{TEGMA}}+\mathrm{DP}_{\mathrm{BuMA}}+\mathrm{DP}_{\mathrm{DMAEMA}}\right) \times\right.$ 
$0.254 \mathrm{~nm}$ ], rather than then size of the random coil which was calculated at $2.7 \mathrm{~nm}$. The experimental value is slightly bigger than the length of a fully extended polymer chain. This could be attributed to the fact that the long side chains of TEGMA units have not been taken into account in the calculations. As opposed to one of our earliest studies [18], in which the size was small enough to ensure no micelle or aggregate formation, in this case, this cannot be verified by the DLS results; this is consistent with our latest study [22]. The difference may be attributed to different compositions but nevertheless, another characterization technique, specifically TEM, was carried out to provide information about the state of the statistical copolymer in solution.

\subsubsection{Transmission Electron Microscopy Images}

To confirm the hypothesis that the block copolymers form spherical micelles and provide more details concerning the state of the statistical copolymer, TEM images were recorded, which are shown in Figure 5.

P1)

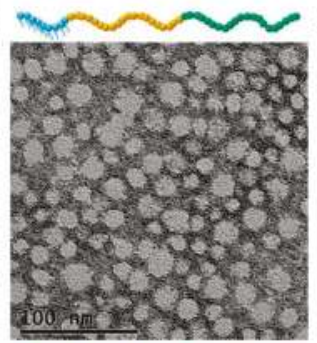

P4)

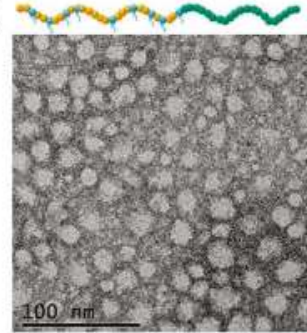

P7)

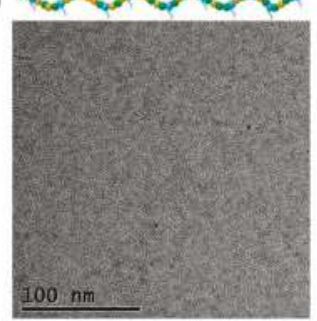

P2)

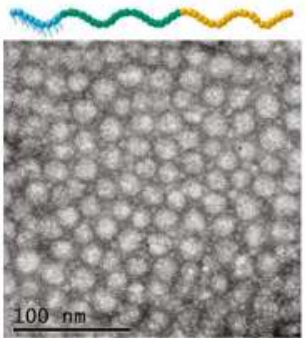

P5)

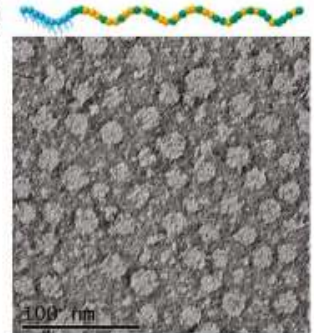

P8)

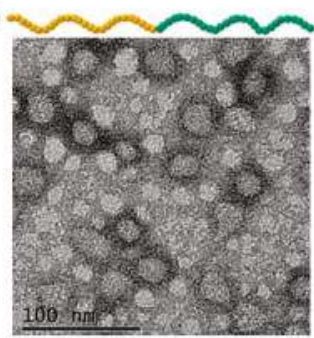

P3)

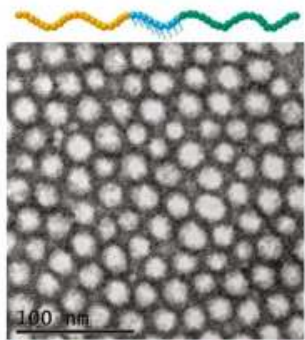

P6)

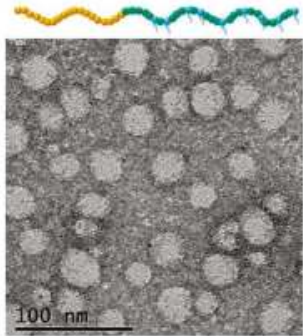

P9)

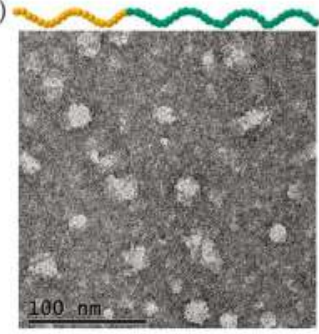

Figure 5. Transmission electron microscopy (TEM) images of $1 \% w / w$ negatively-stained copolymer solutions at $\mathrm{pH}$. The scale bar is set at $100 \mathrm{~nm}$. The schematics of the polymer structures are also given; Blue, orange, and green spheres represent the TEGMA, BuMA, and DMAEMA units, respectively.

As mentioned before, the TEM images at $100 \mathrm{~nm}$ scale are shown in Figure 5 . As a reminder the $\mathrm{pH}$ of the solutions used to prepare the TEM samples was adjusted to 6 to ensure that all polymers were soluble in water. From the TEM results, it can be confirmed that the block copolymers form spherical-like micelles, whereas the statistical copolymer does not. The diameter of these spherical 
micelles in most cases is around $20 \mathrm{~nm}$, with the exception of Polymer 6, the micelles of which appear to be slightly bigger at around $25-30 \mathrm{~nm}$. This is in a good agreement with the DLS data. The only significant difference is observed for Polymer 1, for which the DLS measurements showed a size of $6.5 \mathrm{~nm}$. At this point, it should be remembered that the hydrodynamic diameters are determined as the size corresponding to the maximum intensity on the DLS histograms. In the case of Polymer 1, a bimodal distribution was obtained by DLS, with a second peak at higher values, which confirms the TEM results. The minor differences between the DLS and TEM results can be attributed to the different way of performing the experiments, which was previously discussed [27]. Specifically, DLS is performed in the aqueous state, in which the hydrophobic BuMA units are fully collapsed and the hydrophilic TEGMA and DMAEMA units are fully expanded. On the other hand, TEM is conducted in the dry state [27]. Therefore, it is concluded that the TEM images are not representative of the actual structure of the micelles in solution, since they are recorded in the dry state. However, they give a good approximation of the micelles size and shape, i.e., the size is close to the one obtained by DLS, and formation of spherical-like micelles is revealed by the block copolymers. It is also confirmed by TEM that no micelles are formed by the statistical copolymer, which complements the DLS results.

\subsubsection{Effective $\mathrm{p} K_{\mathrm{a}} \mathrm{s}$}

The effective $\mathrm{p} K_{\mathrm{a}} \mathrm{s}$ of the copolymers, as determined by hydrogen ion titrations, are listed in Table 2 and are shown in Figure 6. In most of the cases, the effective $\mathrm{p} K_{\mathrm{a}}$ values vary between 6.7 and 6.9, which are similar to previously reported $\mathrm{p} K_{\mathrm{a}}$ values on DMAEMA-based polymers [18-22,28-30]. However, the TEGMA $-b$-(BuMA $20-c o-\mathrm{DMAEMA}_{21}$ ) (Polymer 5), the TEGMA $-c o-\mathrm{BuMA}_{20}-\mathrm{co}-\mathrm{DMAEMA}_{21}$ (Polymer 7), and the BuMA $26-b$-DMAEMA 28 (Polymer 8) show lower effective $\mathrm{p} K_{\mathrm{a}} \mathrm{s}$, specifically $6.2,6.1$, and 6.5 , respectively. The low $\mathrm{p} K_{\mathrm{a}}$ of the statistical copolymer is in agreement with other studies on similar statistical terpolymers studied by our group and it is attributed to its inability to form micelles and stabilize itself in solution [18]. This was also proven in this study by TEM. Compared to the previously-studied $\mathrm{PEGMA}_{6}-\mathrm{Co}-\mathrm{BuMA}_{18}-\mathrm{co}-\mathrm{DMAEMA}_{19}$, with the same target $\mathrm{MM}$ and composition, the TEGMA-based copolymer shows significantly lower $\mathrm{p} K_{\mathrm{a}}$ value (6.1 versus 6.7) [22]; this is due to the more hydrophobic TEGMA unit replacing the PEGMA one. The lower $\mathrm{p} K_{\mathrm{a}}$ of Polymer 8 is explained upon considering its higher hydrophobic BuMA content, which decreases the dielectric constant, similar to other studies [19-22,31,32]. Concerning Polymer 5, this is the first systematic study in which this type of architecture has been studied. It can be concluded that randomly copolymerizing the $\mathrm{pH}$-responsive unit DMAEMA with the hydrophobic BuMA one makes the DMAEMA units weaker, based on their steric hindrance and the surrounding hydrophobic environment. Among the other copolymers, it can be observed that the more exposed the DMAEMA units are (see micelle structures in Figure 4), the easier the protonation, thus the stronger the base and the higher the $\mathrm{p} K_{\mathrm{a}}$ values are.

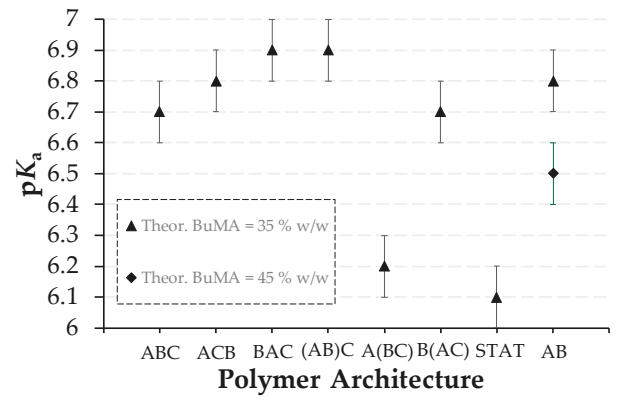

Figure 6. Effect of the polymer architecture and the theoretical hydrophobic BuMA weight percentage on the $\mathrm{p} K_{\mathrm{a}}$. 


\subsubsection{Cloud Points}

Table 2 lists the $\mathrm{CP}$ of $1 \% w / w$ copolymer solutions in DI water when the DMAEMA units were not protonated and were protonated by $10 \%$. At the initial $\mathrm{pH}$ (no protonation), only three of the solutions were homogeneous and thus they were able to be visually tested; specifically, Polymers 1, 4, and 5. The rest of the polymer solutions were insoluble. The insolubility can be ascribed to (i) their architecture (Polymers 2, 3, 6, and 7) and (ii) the increased hydrophobicity of Polymers 8 and 9 (absence of hydrophilic TEGMA and increased hydrophobic content). While Polymer 1 and 5 present a CP at 29 and $32{ }^{\circ} \mathrm{C}$, respectively, Polymer 4 did not respond to temperature, presumably on account of its architecture, which increases the hydrophilicity; the TEGMA groups are distributed in the same block as the hydrophobic BuMA. On the other hand, when the DMAEMA units were protonated by $10 \%$, none of the solutions presents a CP within the temperature range tested. This shows that the protonation of the DMAEMA units, even by only $10 \%$, increases the hydrophilicity of the structure and also prevents aggregation of the micelles on account of the electrostatic repulsion between the positively charged amino groups. The effect of protonation on the thermoresponse of DMAEMA units is well-documented [33,34].

\subsubsection{Visual Gel Points}

Phase diagrams were constructed for seven out of nine polymers. Specifically, the diluted and concentrated solutions of the following polymers in PBS formed homogeneous mixtures (either solutions or gels) and they were visually tested for thermoresponse. The phase diagrams of TEGMA ${ }_{9}-b-\mathrm{BuMA}_{20}-b$-DMAEMA 21 (Polymer 1), $\mathrm{BuMA}_{20}-b-\mathrm{TEGMA}_{9}-b-\mathrm{DMAEMA}_{21}$ (Polymer 3), $\left(\right.$ TEGMA $\left._{9}-c o-\mathrm{BuMA}_{20}\right)-b$-DMAEMA 21 (Polymer 4), TEGMA 9 - $b$-(BuMA 20 -co-DMAEMA 21 ) (Polymer 5), BuMA $_{20}-b$-(TEGMA $-c o-\mathrm{DMAEMA}_{21}$ ) (Polymer 6), TEGMA - -co-BuMA $20-c o-\mathrm{DMAEMA}_{21}$ (Polymer 7), and $\mathrm{BuMA}_{20}-b$-DMAEMA 34 (Polymer 9) are shown in Figure 7. The other two polymers, TEGMA $_{9}-b$-DMAEMA $21-b$-BuMA 20 (Polymer 2) and BuMA $_{26}-b$-DMAEMA 28 (Polymer 8 ), were only soluble at the lowest concentrations and therefore, the construction of meaningful phase diagrams was not feasible.

All the copolymer solutions at $1 \% w / w$ showed a $\mathrm{CP}$ within the temperature range tested, with the exception of Polymer 1. The non-thermoresponse of Polymer 1 can be attributed to the significantly smaller experimental hydrodynamic diameter of the micelles formed by Polymer 1 ; its size is less than half of the size of the micelles formed by the other polymers. While all the copolymer solutions showed thermoresponse at $70{ }^{\circ} \mathrm{C}$ and above, the solution of Polymer 5 interestingly showed thermoresponse at $42^{\circ} \mathrm{C}$. This demonstrates an architectural effect which, by randomly copolymerizing the thermoresponsive unit DMAEMA with the hydrophobic BuMA, while keeping the hydrophilic TEGMA unit as a distinct block, enhances the thermoresponse. This can be explained by considering the enhanced hydrophobic environment surrounding the thermoresponsive unit. This is in contrast with the results obtained by testing the solutions in DI water, during which no thermoresponse was observed. This is due to the ionic strength effect, i.e., screening of the electrostatic repulsions between the protonated DMAEMA units. The ionic strength effect on the LCST of DMAEMA units has been previously observed and discussed by De Souza et al. [33].

Since physical gels have interesting applications, including injectable gels and 3-D printing, the solutions were visually tested for gelation. The gelation region (if there is one), i.e., temperature and concentration ranges where a stable gel was formed, is approximately shown by the black dashed line in Figure 7. The points which correspond to stable transparent and cloudy gel are shown in yellow circles and squares, respectively. Interestingly, a gelation region was observed for five out of six polymers. Specifically, Polymers 1, 3, 5, 6, and 9, formed a stable gel, whereas the solutions of Polymers 4 and 7 only presented a $\mathrm{CP}$, regardless of the concentration of the solution; the formation of a stable gel by the most concentrated solution of the statistical terpolymer can be attributed to reaching the limit of entanglements. The inability of Polymer 4 to form a gel, even in highly concentrated solutions, demonstrates, once again, an architectural effect. Particularly, by randomly copolymerizing the 
hydrophobic BuMA unit with the hydrophilic TEGMA unit, while polymerizing the thermoresponsive DMAEMA in a distinct block, enhances the hydrophilicity of the structure, thus interrupting gelation.

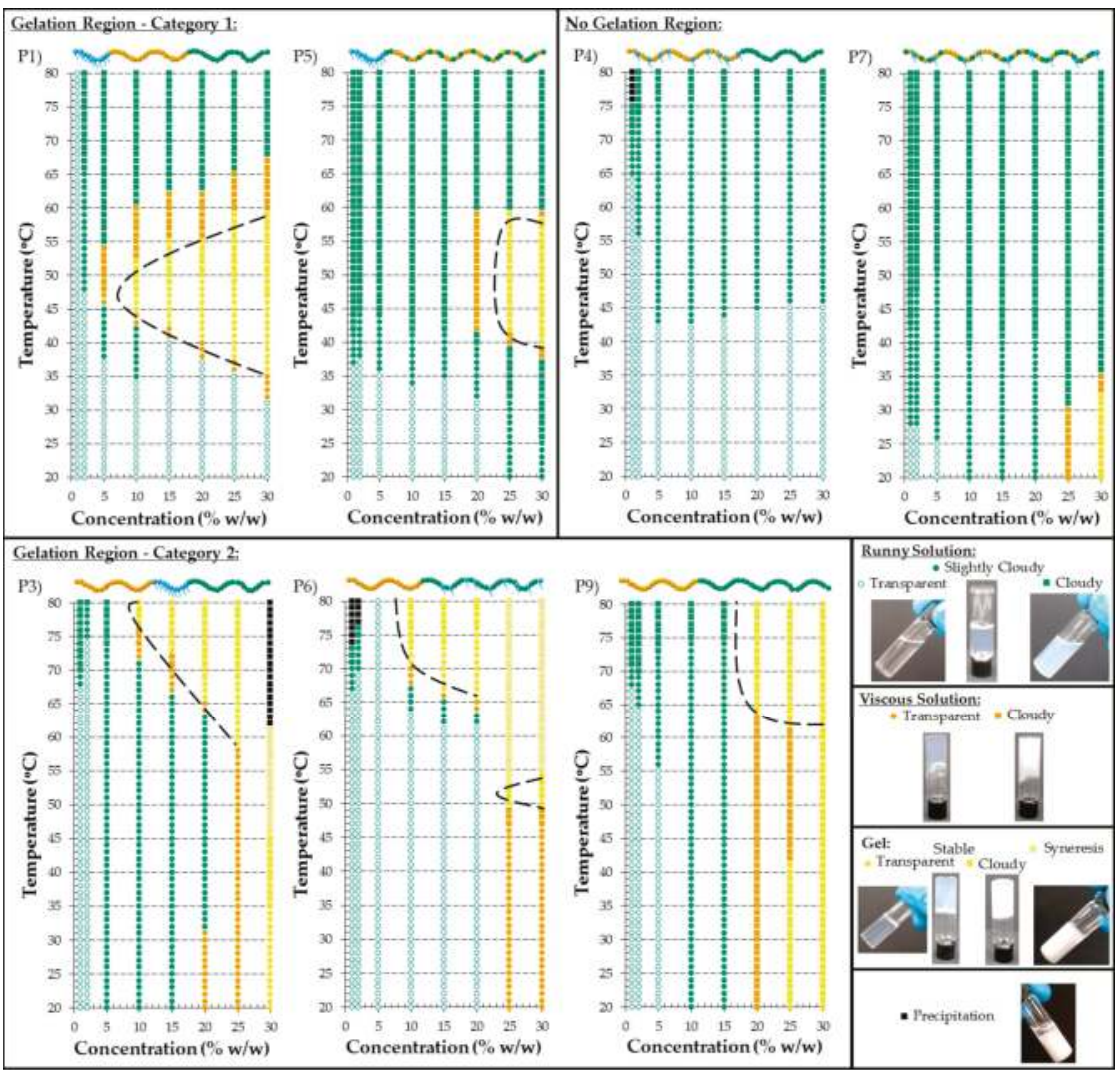

Figure 7. Phase diagrams of the TEGMA-based copolymer solutions in phosphate buffered saline (PBS) of various architectures, namely ABC (Polymer 1), BAC (Polymer 3), (AB)C (Polymer 4), A(BC) (Polymer 5), B(AC) (Polymer 6), and the statistical terpolymer (Polymer 7). The phase diagram of the diblock bipolymer BuMA $20-b$-DMAEMA 34 (Polymer 9) is also shown. The concentrated copolymer solutions in PBS of the ACB (Polymer 2), and the most hydrophobic diblock bipolymer (Polymer 8) were not soluble, thus their phase diagrams are not presented. The transparent and slightly cloudy runny solutions are presented in white and green circles, respectively, whereas the cloudy ones are indicated by green squares. The transparent and cloudy viscous solutions are shown in orange circles and squares, respectively. The transparent and cloudy stable gels are indicated by yellow circles and squares, respectively. Gel syneresis is represented by white squares with diagonal yellow lines, while the precipitation is shown in black squares. The gelation region is approximately shown with the black dashed line. The corresponding polymer structures are schematically given, in which the TEGMA, BuMA, and DMAEMA units are colored in blue, orange, and green, respectively.

The phase diagrams which include a gelation region can be divided into two categories, which differ in the thermoresponsive behavior; as is shown in Figure 7. In the first category, two copolymers are included, specifically Polymers 1 and 5, whereas Polymers 3, 6, and 9 belong to the second category. When copolymer solutions of the first category are concerned, runny solutions are formed at low temperatures, regardless of the increased concentrations. These solutions form a stable gel 
upon increasing the temperature, which then gets destabilized and turns back to solution when the temperature increases further. The ones belonging to the second category, show gelation at higher temperature ranges and the gels formed mostly remain stable until the highest temperature tested. The gelation temperature decreases by increasing the concentration of the solution; as expected. It is worth-noting that in these phase diagrams, the highly concentrated solutions formed either a stable gel or a highly viscous at room temperature. When a stable gel at room temperature was formed, these gels either remained stable upon heating or gel syneresis was observed at higher temperatures. In the case of highly viscous solution, a stable gel is formed upon increasing the temperature, followed by gel syneresis. The gelation at room temperature by highly concentrated polymer solutions can be ascribed to reaching the limit of entanglements rather than to thermoresponse. An interesting feature is that a clear region of transparent gel is not obtained by Polymer 9, which does not contain the hydrophilic TEGMA units and by Polymer 5, in which the thermoresponsive unit is within a hydrophobic BuMA-based environment. Regarding the rest of the polymers which formed a gel (Polymers 1, 3, and 6), a transparent gel was formed which was then transformed into a cloudy one at higher concentrations; this has been previously observed for triblock copolymers containing similar monomers [22].

Therefore, in this study, the effect of architecture on the thermoresponsive behavior is clearly demonstrated. Comparing the behavior of the three triblock terpolymers, the ABC architecture shows clear sol-gel transition, while the ACB one is insoluble and the BAC one shows gelation at higher temperatures, thus confirming the trends previously observed $[18,20]$. Compared to our previous study in which no gelation was observed for the ACB architecture, the gelation in the present study can be attributed to the presence of TEGMA units, instead of PEGMA units (around five repeat EG groups), which increases the hydrophobicity of the structure, thus enhancing the thermoresponse. Concerning the three diblock terpolymers, which have been systematically studied for the first time, the $A(B C)$ architecture shows clear sol-gel transition, whereas the $(A B) C$ one does not form gel, and the $B(A C)$ one forms gel at higher temperatures. On the other hand, no gelation was observed for the statistical terpolymer, which is consistent with our previous studies [18,20]. Interestingly, gelation is observed at high temperatures by the $\mathrm{AB}$ diblock copolymer which did not show solubility issues, specifically Polymer 9, as opposed to the solubility issues of Polymer 8. In conclusion, two architectures, namely $\mathrm{ABC}$ with the hydrophobic BuMA forming the central block and $\mathrm{A}(\mathrm{BC})$ in which the thermoresponsive DMAEMA has been randomly copolymerized with the hydrophobic BuMA, show clear sol-gel-sol transition close to the desirable, body temperature values. Among the two, the ABC architecture is most promising since it shows wider gelation region, i.e., a stable gel is formed at lower temperatures and concentration and this gel gets destabilized at higher temperatures.

\section{Conclusions}

In this study, seven well-defined terpolymers of different architectures were successfully synthesized via GTP. The hydrophilic TEGMA, the hydrophobic BuMA, and the thermoresponsive DMAEMA were used as the A, B, and C units, respectively. The architecture of the polymers was systematically varied in order to investigate its effect on the thermoresponsive behavior of the copolymers. Three architectures, namely $\mathrm{ABC}, \mathrm{ACB}, \mathrm{BAC}$, and statistical, that have been previously studied, as well as three novel architectures, specifically $(\mathrm{AB}) \mathrm{C}, \mathrm{A}(\mathrm{BC})$, and $\mathrm{B}(\mathrm{AC})$ were investigated and compared. Two $\mathrm{BC}$ diblock bipolymers mimicking the BuMA:DMAEMA and BuMA:(TEGMA + DMAEMA) ratios of the weight percentages in the terpolymers were also synthesized. All block copolymers formed spherical micelles unlike the statistical copolymer. Interestingly, the block architecture and the position of the hydrophobic groups strongly influence the thermoresponsive behavior with the $\mathrm{ABC}$ and $\mathrm{A}(\mathrm{BC})$ architectures to show the desirable, clear sol-gel transition close to body temperature.

Supplementary Materials: The following are available online at www.mdpi.com/2073-4360/9/1/31/s1 Figure S1 shows the GPC chromatograms of the triblock terpolymers (Polymers 1-3), the diblock terpolymers (Polymers 4-6), the statistical terpolymer (Polymer 7), the diblock bipolymers (Polymers 8 and 9), and their linear 
precursors. The GPC traces of the first block and the diblock are indicated by blue solid and orange dotted lines, whereas the final triblock terpolymer (if there is any) is shown by green solid line. The GPC chromatogram of the statistical terpolymer is colored in black. Green dashed line represents the GPC traces of the final terpolymers after precipitation; Figure S2 shows the ${ }^{1} \mathrm{H}$ NMR spectra of Polymer 1 before precipitation and its linear precursors: (a) TEGMA, (b) TEGMA $-b-\mathrm{BuMA}_{20}$, and (c) TEGMA $-b-\mathrm{BuMA}_{20}-b-\mathrm{DMAEMA}_{21}$ are colored in blue, orange, and green, respectively.

Acknowledgments: The Department of Materials at Imperial College London is acknowledged for funding a Ph.D. scholarship for Anna P. Constantinou.

Author Contributions: Author Contributions: Anna P. Constantinou performed most of the experiments, analyzed the data, and guided Hanyi Zhao, who assisted with some of the aqueous characterization; Anna P. Constantinou wrote the paper; Catriona M. McGilvery performed the TEM experiments and analysis; Alexandra E. Porter assisted with the TEM analysis and provided the materials for TEM sample preparation; Theoni K. Georgiou conceived the idea, provided the materials, designed the experiments and supervised the work.

Conflicts of Interest: The authors declare no conflict of interest.

\section{References}

1. Constantinou, A.P.; Georgiou, T.K. Tuning the Gelation of Thermoresponsive Gels. Eur. Polym. J. 2016, 78, 366-375. [CrossRef]

2. Ward, M.A.; Georgiou, T.K. Thermoresponsive Polymers for Biomedical Applications. Polymers 2011, 3, 1215-1242. [CrossRef]

3. Chassenieux, C.; Tsitsilianis, C. Recent Trends in pH/Thermo-Responsive Self-Assembling Hydrogels: From Polyions to Peptide-Based Polymeric Gelators. Soft Matter 2016, 12, 1344-1359. [CrossRef] [PubMed]

4. Southall, N.T.; Dill, K.A.; Haymet, A.D.J. A View of the Hydrophobic Effect. J. Phys. Chem. B 2002, 106, 521-533. [CrossRef]

5. Madsen, J.; Armes, S.P.; Bertal, K.; Lomas, H.; MacNeil, S.; Lewis, A.L. Biocompatible Wound Dressings Based on Chemically Degradable Triblock Copolymer Hydrogels. Biomacromolecules 2008, 9, 2265-2275. [CrossRef] [PubMed]

6. Place, E.S.; George, J.H.; Williams, C.K.; Stevens, M.M. Synthetic Polymer Scaffolds for Tissue Engineering. Chem. Soc. Rev. 2009, 38, 1139-1151. [CrossRef] [PubMed]

7. Elstad, N.L.; Fowers, K.D. OncoGel (ReGel/Paclitaxel)—Clinical Applications for a Novel Paclitaxel Delivery System. Adv. Drug Deliv. Rev. 2009, 61, 785-794. [CrossRef] [PubMed]

8. Zentner, G.M.; Rathi, R.; Shih, C.; McRea, J.C.; Seo, M.-H.; Oh, H.; Rhee, B.G.; Mestecky, J.; Moldoveanu, Z.; Morgan, M.; et al. Biodegradable Block Copolymers for Delivery of Proteins and Water-Insoluble Drugs. J. Control. Release 2001, 72, 203-215. [CrossRef]

9. Hsieh, F.-Y.; Lin, H.-Y.; Hsu, S.-H. 3D Bioprinting of Neural Stem Cell-Laden Thermoresponsive Biodegradable Polyurethane Hydrogel and Potential in Central Nervous System Repair. Biomaterials 2015, 71, 48-57. [CrossRef] [PubMed]

10. Tsai, Y.-C.; Li, S.; Hu, S.-G.; Chang, W.-C.; Jeng, U.-S.; Hsu, S.-H. Synthesis of Thermoresponsive Amphiphilic Polyurethane Gel as a New Cell Printing Material near Body Temperature. ACS Appl. Mater. Interfaces 2015, 7, 27613-27623. [CrossRef] [PubMed]

11. Zhang, M.; Vora, A.; Han, W.; Wojtecki, R.J.; Maune, H.; Le, A.B.A.; Thompson, L.E.; McClelland, G.M.; Ribet, F.; Engler, A.C.; et al. Dual-Responsive Hydrogels for Direct-Write 3D Printing. Macromolecules 2015, 48, 6482-6488. [CrossRef]

12. Kolesky, D.B.; Truby, R.L.; Gladman, A.S.; Busbee, T.A.; Homan, K.A.; Lewis, J.A. 3D Bioprinting of Vascularized, Heterogeneous Cell-Laden Tissue Constructs. Adv. Mater. 2014, 26, 3124-3130. [CrossRef] [PubMed]

13. Buwalda, S.J.; Boere, K.W.M.; Dijkstra, P.J.; Feijen, J.; Vermonden, T.; Hennink, W.E. Hydrogels in a Historical Perspective: From Simple Networks to Smart Materials. J. Control. Release 2014, 190, 254-273. [CrossRef] [PubMed]

14. Skardal, A.; Atala, A. Biomaterials for Integration with 3-D Bioprinting. Ann. Biomed. Eng. 2015, 43, 730-746. [CrossRef] [PubMed]

15. Davis, F.J. Polymer Chemistry: A Practical Approach; Oxford University Press: Oxford, UK, 2004. 
16. Webster, O.W.; Hertler, W.R.; Sogah, D.Y.; Farnham, W.B.; RajanBabu, T.V. Group-Transfer Polymerization. 1. A New Concept for Addition Polymerization with Organosilicon Initiators. J. Am. Chem. Soc. 1983, 105, 5706-5708. [CrossRef]

17. Hatada, K.; Kitayama, T.; Vogl, O. Macromolecular Desing of Polymeric Materials; Marcel Dekker, Inc.: New York, NY, USA, 1997; p. 878.

18. Ward, M.A.; Georgiou, T.K. Multicompartment Thermoresponsive Gels: Does the Length of the Hydrophobic Side Group Matter? Polym. Chem. 2013, 4, 1893-1902. [CrossRef]

19. Ward, M.A.; Georgiou, T.K. Thermoresponsive Gels Based on ABA Triblock Copolymers: Does the Asymmetry Matter? J. Polym. Sci. A 2013, 51, 2850-2859. [CrossRef]

20. Ward, M.A.; Georgiou, T.K. Thermoresponsive Terpolymers Based on Methacrylate Monomers: Effect of Architecture and Composition. J. Polym. Sci. A 2010, 48, 775-783. [CrossRef]

21. Ward, M.A.; Georgiou, T.K. Thermoresponsive Triblock Copolymers Based on Methacrylate Monomers: Effect of Molecular Weight and Composition. Soft Matter 2012, 8, 2737-2745. [CrossRef]

22. Constantinou, A.P.; Georgiou, T.K. Thermoresponsive Gels Based on ABC Triblock Copolymers: Effect of the Length of the PEG Side Group. Polym. Chem. 2016, 7, 2045-2056. [CrossRef]

23. Dicker, I.B.; Cohen, G.M.; Farnham, W.B.; Hertler, W.R.; Laganis, E.D.; Sogah, D.Y. Oxyanions Catalyze Group-Transfer Polymerization to Give Living Polymers. Macromolecules 1990, 23, 4034-4041. [CrossRef]

24. Patrickios, C.S.; Lowe, A.B.; Armes, S.P.; Billingham, N.C. ABC Triblock Polymethacrylates: Group Transfer Polymerization Synthesis of the $\mathrm{ABC}, \mathrm{ACB}$, and $\mathrm{BAC}$ Topological Isomers and Solution Characterization. J. Polym. Sci. A 1998, 36, 617-631. [CrossRef]

25. Raduan, N.H.; Horozov, T.S.; Georgiou, T.K. “Comb-Like” Non-Ionic Polymeric Macrosurfactants. Soft Matter 2010, 6, 2321-2329. [CrossRef]

26. Hadjiyannakou, S.C.; Vamvakaki, M.; Patrickios, C.S. Synthesis, Characterization and Evaluation of Amphiphilic Diblock Copolymer Emulsifiers Based on Methoxy Hexa(Ethylene Glycol) Methacrylate and Benzyl Methacrylate. Polymer 2004, 45, 3681-3692. [CrossRef]

27. Wu, C.; Ying, A.; Ren, S. Fabrication of Polymeric Micelles with Core-Shell-Corona Structure for Applications in Controlled Drug Release. Colloid Polym. Sci. 2013, 291, 827-834. [CrossRef]

28. Georgiou, T.K.; Vamvakaki, M.; Patrickios, C.S.; Yamasaki, E.N.; Phylactou, L.A. Nanoscopic Cationic Methacrylate Star Homopolymers: Synthesis by Group Transfer Polymerization, Characterization and Evaluation as Transfection Reagents. Biomacromolecules 2004, 5, 2221-2229. [CrossRef] [PubMed]

29. Georgiou, T.K.; Phylactou, L.A.; Patrickios, C.S. Synthesis, Characterization, and Evaluation as Transfection Reagents of Ampholytic Star Copolymers: Effect of Star Architecture. Biomacromolecules 2006, 7, 3505-3512. [CrossRef] [PubMed]

30. Simmons, M.R.; Patrickios, C.S. Synthesis and Aqueous Solution Characterization of Catalytically Active Block Copolymers Containing Imidazole. Macromolecules 1998, 31, 9075-9077. [CrossRef]

31. Emileh, A.; Vasheghani-Farahani, E.; Imani, M. Swelling Behavior, Mechanical Properties and Network Parameters of $\mathrm{pH}$ - and Temperature-Sensitive Hydrogels of Poly((2-Dimethyl Amino) Ethyl Methacrylate-coButyl Methacrylate). Eur. Polym. J. 2007, 43, 1986-1995. [CrossRef]

32. Philippova, O.E.; Hourdet, D.; Audebert, R.; Khokhlov, A.R. pH-Responsive Gels of Hydrophobically Modified Poly(Acrylic Acid). Macromolecules 1997, 30, 8278-8285. [CrossRef]

33. De Souza, J.C.P.; Naves, A.F.; Florenzano, F.H. Specific Thermoresponsiveness of PMMA-Block-PDMAEMA to Selected Ions and Other Factors in Aqueous Solution. Colloid Polym. Sci. 2012, 290, 1285-1291. [CrossRef]

34. Fournier, D.; Hoogenboom, R.; Thijs, H.M.L.; Paulus, R.M.; Schubert, U.S. Tunable pH- and TemperatureSensitive Copolymer Libraries by Reversible Addition-Fragmentation Chain Transfer Copolymerizations of Methacrylates. Macromolecules 2007, 40, 915-920. [CrossRef] 
Article

\title{
Semi-Interpenetrating Polymer Networks with Predefined Architecture for Metal Ion Fluorescence Monitoring
}

\author{
Kyriakos Christodoulou ${ }^{1}$, Epameinondas Leontidis ${ }^{2}$, Mariliz Achilleos ${ }^{1}$, Christiana Polydorou ${ }^{1}$ \\ and Theodora Krasia-Christoforou ${ }^{1, *}$ \\ 1 Department of Mechanical and Manufacturing Engineering, University of Cyprus, 1678 Nicosia, Cyprus; \\ christodoulou.c.kyriakos1@ucy.ac.cy (K.C.); achilleos.mariliz@ucy.ac.cy (M.A.); \\ polydorou.christiana@ucy.ac.cy (C.P.) \\ 2 Department of Chemistry, University of Cyprus, 1678 Nicosia, Cyprus; psleon@ucy.ac.cy \\ * Correspondence: krasia@ucy.ac.cy; Tel.: +357-2289-2288
}

Academic Editor: Alexander Böker

Received: 26 September 2016; Accepted: 23 November 2016; Published: 29 November 2016

\begin{abstract}
The development of new synthetic approaches for the preparation of efficient 3D luminescent chemosensors for transition metal ions receives considerable attention nowadays, owing to the key role of the latter as elements in biological systems and their harmful environmental effects when present in aquatic media. In this work, we describe an easy and versatile synthetic methodology that leads to the generation of nonconjugated 3D luminescent semi-interpenetrating amphiphilic networks (semi-IPN) with structure-defined characteristics. More precisely, the synthesis involves the encapsulation of well-defined poly(9-anthrylmethyl methacrylate) (pAnMMA) (hydrophobic, luminescent) linear polymer chains within a covalent poly(2-(dimethylamino)ethyl methacrylate) (pDMAEMA) hydrophilic polymer network, derived via the 1,2-bis-(2-iodoethoxy)ethane (BIEE)-induced crosslinking process of well-defined pDMAEMA linear chains. Characterization of their fluorescence properties demonstrated that these materials act as strong blue emitters when exposed to UV irradiation. This, combined with the presence of the metal-binding tertiary amino functionalities of the PDMAEMA segments, allowed for their applicability as sorbents and fluorescence chemosensors for transition metal ions $\left(\mathrm{Fe}^{3+}, \mathrm{Cu}^{2+}\right)$ in solution via a chelation-enhanced fluorescence-quenching effect promoted within the semi-IPN network architecture. Ethylenediaminetetraacetic acid (EDTA)-induced metal ion desorption and thus material recyclability has been also demonstrated.
\end{abstract}

Keywords: semi-interpenetrating networks; fluorescent networks; anthracene; metal ions; fluorescence sensors

\section{Introduction}

The development of luminescent chemosensors for transition metal ions has attracted considerable attention in the last years given their high importance in biological systems [1], but also their harmful environmental effects when they are present in high concentrations in aquatic media [2,3]. To ensure high efficiency in a fluorescent metal-ion sensor, the light-emitting moiety should be covalently linked to a metal ion-binding group, and its fluorescence properties must be sensitive to the ion-ligand interaction $[4,5]$.

Most literature examples focusing on 3D luminescent materials that are used as sensor platforms deal with metal-organic frameworks (MOFs) [6-15] and 3D coordination polymers [16-20]. Only a few research groups have been working on the synthesis of fluorescent semi-interpenetrating network (semi-IPN) architectures. The latter are 3D polymer structures consisting of secondary linear polymer 
chains that are interlaced—but not covalently bonded —with a primary polymer network [21-24]. In this limited number of existing reports, the fluorescent component is either a conjugated conductive polymer [25-28], fluorescent nanoparticles such as carbon nanodots and quantum dots (QDs) [29-31], or fluorescent dyes dispersed within or covalently linked to the polymer matrix [32-35]. However, the abovementioned materials have several disadvantages including: (a) low structural stability and robustness in the presence of chemical and physical impacts [36-40]; (b) possible leaching and relatively high probability of aggregation-induced fluorescence quenching of the physically entrapped organic dyes [41]; and (c) agglomeration phenomena of the fluorescent nanoparticles such as carbon nanodots resulting in inferior fluorescence properties due to self-quenching [42].

The necessity of developing functional organic polymer networks with controllable architectures have directed polymer chemists to explore new synthetic routes towards this purpose. Such materials are considered to be highly advantageous in comparison to their analogues prepared by a noncontrolled chemical crosslinking process, since the latter are usually highly inhomogeneous owing to the non-precise molar mass and broad molar mass distributions of the polymer segments constituting the networks. Consequently, polymer networks characterized by ill-defined architectures usually exhibit inferior mechanical and swelling properties, whereas their structural and compositional inhomogeneities restrict the structure-to-property correlation [43].

Although numerous examples on new synthetic approaches resulting in the generation of "model" or "quasi-model" covalent polymer networks have appeared so far, including "quasi-living" carbocationic polymerization [44,45], anionic polymerization [46,47], group transfer polymerization (GTP) [48-50], and controlled radical polymerization processes [51-54], there is only a limited number of publications discussing the synthesis of semi-IPN exhibiting structure-defined characteristics $[55,56]$. Very recently, our group has reported on the synthesis of such materials [57]. These consisted of well-defined hydrophilic and pH-responsive (poly(2-dimethylamino) ethyl methacrylate) (pDMAEMA) linear chains that were interconnected using 1,2-bis-(2-iodoethoxy)-ethane (BIEE) generating the pDMAEMA network, and well-defined hydrophobic poly ( $n$-butyl methacrylate) (pBuMA) linear chains that were encapsulated within the network during the crosslinking process. From our studies, it has been demonstrated that the mechanical properties of these materials can be easily tuned by adjusting the content of the encapsulated hydrophobic linear chains, whereas their well-defined structural characteristics allowed for the prediction of their mechanical response via mathematical modeling.

Giving further credence to the BIEE-crosslinking approach as an alternative to synthetically demanding and multistep controlled polymerization processes, in the present study we report on the synthesis of 3D structure-defined emissive (fluorescent) amphiphilic semi-IPN, consisting of BIEE-crosslinked pDMAEMA segments and embedded hydrophobic and nonconjugated/fluorescent poly(9-anthrylmethyl methacrylate) (pAnMMA) linear chains both prepared by reversible addition-fragmentation chain transfer (RAFT)-controlled radical polymerization. Although in this case the coordinating active part (pDMAEMA) [58-63] is not covalently bound to the fluorescent active component (pAnMMA), the semi-IPN network architecture promotes the reinforcement of interactions between the pDMAEMA-complexed transition metal ions and the anthracene moieties of the interlaced pAnMMA chains, thus promoting chelation-enhanced fluorescence quenching. Moreover, interlacing of the pAnMMA chains within the nonfluorescent pDMAEMA network diminishes phase separation phenomena (and, consequently, self-quenching effects). Furthermore, the high molar mass and hydrophobicity of the macromolecular fluorescent pAnMMA prevents the leaching of the active component in polar and aqueous solvents.

These materials were further evaluated in the chemosensing of $\mathrm{Fe}^{3+}$ and $\mathrm{Cu}^{2+}$ transition metal ions. The former is a key element in biological and environmental systems, playing an essential role in oxygen uptake and metabolism and electron transfer processes [64]. $\mathrm{Cu}^{2+}$ ions, which are known to exhibit high affinity for $\mathrm{N}$ - and $\mathrm{O}$-containing ligands, significantly contribute to the metal environmental pollution owing to their widespread industrial use. Even though the toxicity of $\mathrm{Cu}^{2+}$ 
ions is considerably lower compared to other heavy metal ions, very low $\mathrm{Cu}^{2+}$ concentrations are highly toxic to certain microorganisms [65].

Consequently, the presented versatile synthetic approach creates new prospects in the generation of nonconjugated 3D luminescent polymer-based sensors in which fluorescent moieties are combined with metal-chelating elements, characterized by structure-defined characteristics and tunable properties, with potential use in metal ion chemosensing.

\section{Materials and Methods}

\subsection{Chemical Reagents}

Poly(9-Anthrylmethyl methacrylate) (pAnMMA) $\left(M_{n}=27,900 \mathrm{~g} \cdot \mathrm{mol}^{-1}\right.$; where $M_{n}$ : number average molar mass determined by size exclusion chromatography using poly(methyl methacrylate) (PMMA) calibration standards; polydispersity index, PDI: 1.3) and poly(2-(dimethylamino)ethyl methacrylate) (pDMAEMA) $\left(M_{\mathrm{n}}: 19,000 \mathrm{~g} \cdot \mathrm{mol}^{-1}\right.$, polydispersity index, PDI: 1.17), were in-house synthesized by RAFT-controlled radical polymerization according to our previous publications [5]. 1,2-bis-(2-iodothoxy)ethane (BIEE, Sigma-Aldrich, 96\%, St. Louis, MO, USA), 9-anthracenemethanol (Sigma-Aldrich, 97\%, St. Louis, MO, USA), methanol (MeOH, Sharlau, analytical grade, ACS reagent, Barcelona, Spain), and tetrahydrofuran (THF, Scharlau, HPLC grade, Barcelona, Spain) were used as received. $\mathrm{FeCl}_{3} \cdot 6 \mathrm{H}_{2} \mathrm{O}$ (Sigma-Aldrich, $\geq 99 \%$, St. Louis, $\mathrm{MO}, \mathrm{USA}$ ), $\mathrm{Cu}\left(\mathrm{CH}_{3} \mathrm{COO}\right)_{2} \cdot \mathrm{H}_{2} \mathrm{O}$ (Sigma-Aldrich, $\geq 99 \%$, St. Louis, MO, USA) and ethylenediaminetetraacetic acid (EDTA) (Sharlau, $99 \%-101 \%$, Barcelona, Spain) were used as received by the supplier.

\subsection{Synthesis of Semi-Interpenetrating BIEE-Crosslinked $p A n M M A / p D M A E M A$ Networks}

By following a similar synthetic protocol as that described in a recent publication of our group [57], a semi-interpenetrating fluorescent polymer network of the type $\mathrm{p}$ (AnMMA)/pDMAEMA/BIEE was prepared via the encapsulation of pAnMMA ( $5 \mathrm{wt} \%$ in respect to the total polymer mass) within the BIEE-crosslinked pDMAEMA network. The experimental procedure is as follows. In a glass vial, pAnMMA ( $5 \mathrm{mg}$ ) was dissolved in THF ( $1.25 \mathrm{~mL}, 8 \% w / v$ solution concentration). To the solution, pDMAEMA (100 mg, $0.0052 \mathrm{mmol}$ of macro-chain transfer agent, $0.64 \mathrm{mmol}$ per DMAEMA unit) was added and the mixture was stirred rapidly until complete dissolution of pDMAEMA. To the solution, BIEE ( $58 \mu \mathrm{L}, 118 \mathrm{mg}, 0.32 \mathrm{mmol}$ ) was added using a micropipette. The resulting solution was stirred rapidly and was then left in a sealed vial at room temperature, under air and without stirring, until gelation was observed (7 days). The resulting network was then placed in excess methanol $(100 \mathrm{~mL})$ for one week to remove the sol fraction. The sol fraction (14\%) was determined gravimetrically, and it was calculated from the ratio of the dried mass of the extractables to the theoretical mass of all components in the network (i.e., pAnMMA, pDMAEMA, and BIEE).

\subsection{Swelling Behavior}

The methanol-swollen network was cut into small pieces and their mass was determined gravimetrically before placing them in a vacuum oven to dry at ca. $25^{\circ} \mathrm{C}$ for $24 \mathrm{~h}$. The mass of the dry network pieces was then determined, and the degrees of swelling (DSs) in $\mathrm{MeOH}$ were calculated as the ratio of the swollen mass divided by the dry mass. Subsequently, the dried pieces were placed in deionized water for 2 weeks before determining the water-swollen network masses.

\subsection{Fluorescent Characterization}

The fluorescence emission spectrum of pre-swollen (in methanol) polymer network was recorded at the solid state by using a Jasco FP-6300 fluorescence spectrophotometer (Jasco Incorporated, Easton, MD, USA). The excitation wavelength was set at $370 \mathrm{~nm}$, where AnMMA-containing polymers are known to exhibit maximum absorption [66]. Fluorescence microscopy was further used for visualizing the anthracene-containing fluorescent network at its swollen state (in methanol). The swollen network 
was placed in methanol-containing Petri dishes and examined under the Olympus fluorescence microscope (BX53 System Microscope, Olympus Corporation, Tokyo, Japan). The fluorescence intensity of the sample was determined by using the DAPI filter (U-FUNA, excitation: $358 \mathrm{~nm}$, emission: $461 \mathrm{~nm}$; Olympus, Tokyo, Japan). Other filters were used with different excitation and emission spectra, but no fluorescence was detected. Images were taken at $4 \times$ magnification and analyzed using the CellSens software. The same exposure time was used in all cases.

\subsection{Fluorescence Monitoring of $\mathrm{Cu}^{2+}$ and $\mathrm{Fe}^{3+}$ Ions}

Initially, $\mathrm{Fe}^{3+}$ and $\mathrm{Cu}^{2+}$ metal ion solutions of various concentrations were prepared by dissolving $\mathrm{FeCl}_{3} \cdot 6 \mathrm{H}_{2} \mathrm{O}$ and $\mathrm{Cu}\left(\mathrm{CH}_{3} \mathrm{COO}\right)_{2} \cdot \mathrm{H}_{2} \mathrm{O}$ metal ion salts in methanol. More precisely, $\mathrm{Fe}^{3+}$ and $\mathrm{Cu}^{2+}$ methanol solutions were prepared, with concentrations in the range $5 \times 10^{-6}$ to $5 \times 10^{-5} \mathrm{M}$ and $5 \times 10^{-5}$ to $10^{-3} \mathrm{M}$, respectively.

The polymer network was cut in small pieces ( $\mathrm{m} \sim 0.70 \mathrm{~g}$ ), which were subsequently immersed in glass vials containing the metal ion $\left(\mathrm{Fe}^{3+}\right.$ and $\left.\mathrm{Cu}^{2+}\right)$ solutions $(5 \mathrm{~mL})$ for $24 \mathrm{~h}$. For comparison purposes, a control sample was also prepared upon immersing the polymer network in a glass vial containing pure methanol $(5 \mathrm{~mL})$. Afterwards, the pieces were washed twice with pure methanol and placed in new vials containing methanol $(5 \mathrm{~mL})$ prior to their characterization.

For comparison purposes, the fluorescence monitoring of $\mathrm{Fe}^{3+}$ and $\mathrm{Cu}^{2+}$ in methanol (concentration range $0.1-0.25 \mathrm{mM}\left(\mathrm{Fe}^{3+}\right)$ and $0.1-1.0 \mathrm{mM}\left(\mathrm{Cu}^{2+}\right)$ ) was carried out by using the low molar mass 9-anthracenemethanol $(1.25 \mathrm{mM})$ as the metal ion fluorescent chemosensor.

\subsection{Desorption Studies-Polymer Network Regeneration}

Initially, $\mathrm{Cu}^{2+}$-loaded polymer networks were prepared by immersing the methanol-swollen $\mathrm{p}\left(\right.$ AnMMA)/pDMAEMA/BIEE networks in $\mathrm{Cu}^{2+}$ ion methanol solutions of two different $\mathrm{Cu}^{2+}$ concentrations $\left(10^{-3}\right.$ and $\left.5 \times 10^{-3} \mathrm{M}\right)$ for $24 \mathrm{~h}$ followed by extensive washing to remove any unbound cations. For the regeneration process, the $\mathrm{Cu}^{2+}$-loaded polymer network pieces $(\mathrm{m} \sim 0.35 \mathrm{~g}$ ) were immersed in aqueous EDTA solution $(5 \mathrm{~mL}, 0.25 \mathrm{M}$ ) and they were visualized by fluorescence microscopy in real time.

\section{Results and Discussion}

\subsection{Synthesis of Semi-Interpenetrating 3D Amphiphilic Fluorescent Networks}

The synthetic methodology followed for the preparation of structure-defined, BIEE-crosslinked fluorescent semi-IPN polymer networks was based on our recent publication [57]. Initially, pDMAEMA and fluorescent poly(AnMMA) linear homopolymers were prepared by RAFT-controlled radical polymerization. Unimodal polymers with controlled average molar mass (MWs) and relatively low PDIs were obtained in both cases [5,57]. The well-defined poly(DMAEMA) and poly(AnMMA) linear precursors were then dissolved in tetrahydrofuran (i.e., a good solvent for both homopolymers to ensure good intermixing at a molecular level) and employed as precursors for the generation of the $\mathrm{BIEE} / \mathrm{pDMAEMA} / \mathrm{pAnMMA}$ semi-IPN polymer networks, as schematically demonstrated in Figure 1.

The gelation process that was carried out at room temperature, under air and without mechanical stirring, was completed within 7 days. As already described in Section 2.2, the [BIEE]/[DMAEMA] molar ratio was fixed at 1:2, targeting a 100\% degree of crosslinking, since 1 BIEE molecule is capable of linking together 2 tertiary amino functionalities. However, according to Armes and co-workers [67], intrachain crosslinking or reaction of one iodide group of the BIEE crosslinker may occur as a result of deviation from quantitative crosslinking. More precisely, quaternization of the PDMAEMA chains with BIEE may lead to either intermolecular crosslinking (branching) or intramolecular cyclization, as shown in Figure 1b. The sol fraction percentage (extractables) that was determined gravimetrically was $14 \%$, which is in line with that reported in our previous study [57]. 
The networks' swelling behavior is considered to be an important influencing parameter on their performance in metal ion uptake and sensing. The swelling behavior of the BIEE-crosslinked $\mathrm{p}$ (AnMMA)/pDMAEMA networks was investigated in both, water, and methanol. Based on the experimental data, the degree of swelling determined in water (19.1 \pm 1.7$)$ was comparable to that found in methanol (16.3 \pm 0.8$)$, suggesting that shifting from methanol to water would probably not lead to significant changes on the network's sensing performance.

(a)

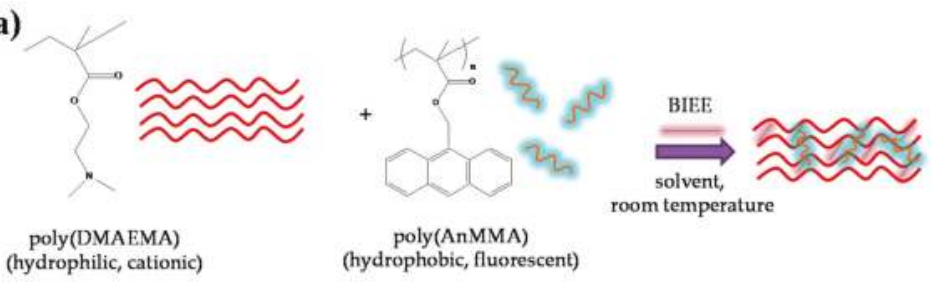

BIEE: $1 \mathrm{CH}_{2} \mathrm{CH}_{2} \mathrm{OCH}_{2} \mathrm{CH}_{2} \mathrm{OCH}_{2} \mathrm{CH}_{2} \mathrm{I}$

(b)

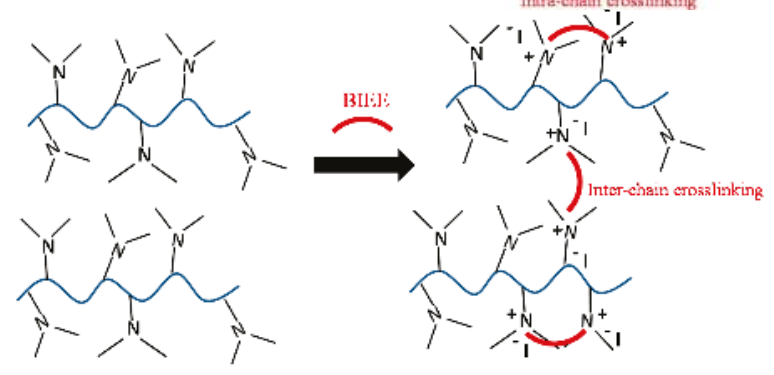

Figure 1. (a) Chemical structures of the linear poly(2-(dimethylamino)ethyl methacrylate) (pDMAEMA) and poly(9-anthrylmethyl methacrylate) (pAnMMA) homopolymers and of the 1,2-bis-(2-iodoethoxy)ethane (BIEE) crosslinking agent, and schematic of the one-step synthetic approach followed for the preparation of 3D luminescent semi-interpenetrating amphiphilic network (APN); (b) crosslinking reaction scheme presenting both, intra- and interchain crosslinking pathways that may occur between BIEE and $-\mathrm{N}(\mathrm{Me})_{3}$ pendant moieties of the poly(DMAEMA) linear chains.

\subsection{Fluorescence Properties}

The fluorescence emission of the network pre-swollen in methanol was investigated by means of fluorescence spectroscopy and microscopy. As previously described in the experimental section, a BIEE/pDMAEMA/pAnMMA semi-IPN polymer network was synthesized, in which 5 wt $\%$ of the fluorescent pAnMMA component with respect to the total mass (polymers + crosslinking agent) was incorporated. As seen in Figure 2, the resulting network displayed strong blue emission (recorded at $461 \mathrm{~nm}$ ) under $358 \mathrm{~nm}$ excitation wavelength. Moreover, the active fluorescent polymeric component was homogeneously distributed within the pDMAEMA polymer matrix, as verified by fluorescence microscopy.

Fluorescence spectroscopy was used for recording the fluorescence emission spectrum of the methanol-swollen network. The vibronic structure of the network provided in Figure 3 resembled that of the anthracene fluorophore $[66,68]$ and of the previously reported pAnMMA linear homopolymer analogue [5]. 

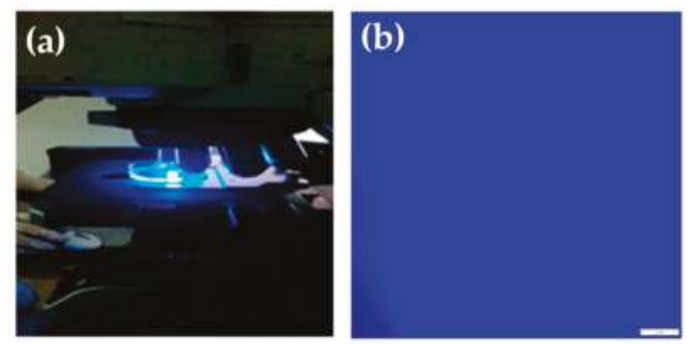

Figure 2. (a) Photograph of the BIEE/pDMAEMA/pAnMMA semi-interpenetrating network (semi-IPN) polymer network when exposed to UV irradiation, displaying strong blue emission; (b) corresponding fluorescence microscopy image. The scale bar in Figure $2 \mathrm{~b}$ is $100 \mu \mathrm{m}$.

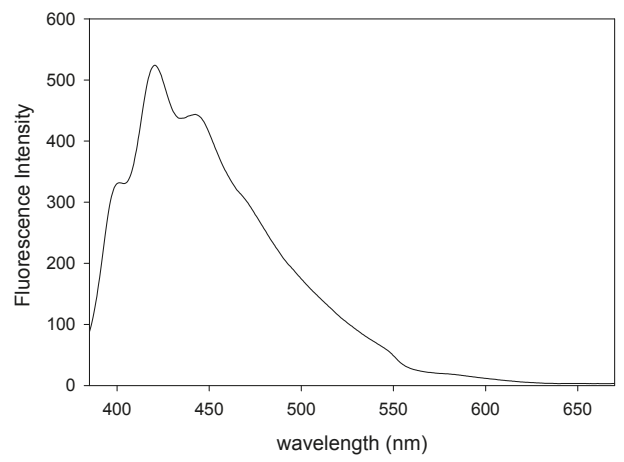

Figure 3. Fluorescence spectrum of the BIEE/pDMAEMA/pAnMMA semi-IPN polymer network pre-swollen in methanol. Excitation wavelength: $370 \mathrm{~nm}$.

\subsection{Fluorescence Monitoring of $\mathrm{Cu}^{2+}$ and $\mathrm{Fe}^{3+}$ Ions}

The BIEE/pDMAEMA/pAnMMA semi-IPN polymer network system was examined regarding its ability to act as a macromolecular sensor for transition metal ions. More precisely, the study was focused on examining the chemosensing ability of such fluorescent 3D semi-IPN towards $\mathrm{Fe}^{3+}$ and $\mathrm{Cu}^{2+}$ ions dissolved in methanol. Both fluorescence microscopy and spectroscopy were used to monitor the qualitative and quantitative changes in the network's fluorescence intensity when the latter was immersed in metal ion solutions of various concentrations prepared in methanol. The metal ion fluorescence monitoring studies were purposely performed in methanol, since this allowed for a direct comparison of the obtained fluorescence data with those acquired when using 9-anthracenemethanol (model compound) as a fluorophore (see Supplementary Materials), which is insoluble in water. Moreover, based on previous literature reports [69-73], the use of methanol or methanol/water mixtures of various volume ratios is typical in metal ion fluorescence monitoring studies, since methanol-being a protic and very polar solvent-exhibits properties similar to water. In one such example [73], the authors performed selective fluorescence quenching experiments by using an aziridine-based molecule possessing pendant anthracene units as a chemosensor, in both methanol and water as solvents, so as to determine the solvent effect on the quenching/enhancement mechanism. No significant differences were found in regards to the quenching of the fluorescence intensity in the presence of different metal ions when using water or methanol as solvents.

Figure $4 \mathrm{a}$ provides the fluorescence images corresponding to the BIEE/pDMAEMA/pAnMMA methanol-swollen network immersed in pure methanol (control samples) and methanol solutions containing different $\mathrm{Fe}^{3+}$ and $\mathrm{Cu}^{2+}$ metal ion concentrations. From the obtained fluorescence images, 
it can be clearly observed that the fluorescence intensity of the network is effectively quenched in the presence of both $\mathrm{Fe}^{3+}$ and $\mathrm{Cu}^{2+}$ metal ions, whereas quenching is more pronounced upon increasing the metal ion concentration as expected. During the analysis by means of fluorescence microscopy, photographs of the networks immersed in metal ion solutions of various concentrations were also taken in real time. As seen in Figure $4 b$, fluorescence quenching can be easily visualized, since upon immersion of the fluorescent network in the metal ion solutions its fluorescence efficiency reduces significantly.

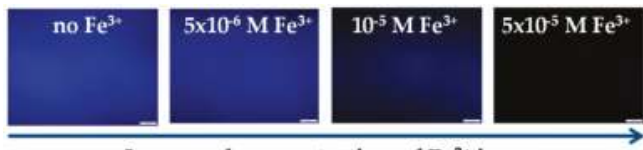

Increased concentration of $\mathrm{Fe}^{3+}$ ions

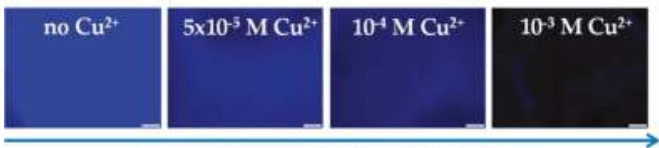

Increased concentration of $\mathrm{Cu}^{2+}$ ions
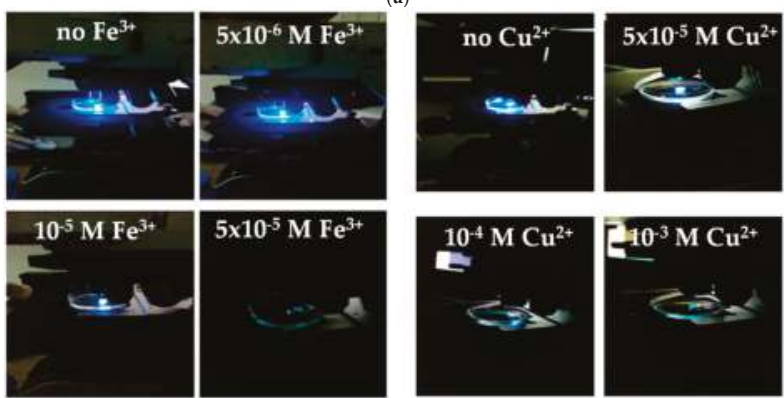

(b)

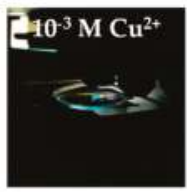

Figure 4. (a) Fluorescence images of the methanol-swollen BIEE/pDMAEMA/pAnMMA network immersed in pure methanol (control samples) and methanol solutions containing different $\mathrm{Fe}^{3+}$ and $\mathrm{Cu}^{2+}$ metal ion concentrations; (b) corresponding photographs. The scale bar in Figure 4a is $100 \mu \mathrm{m}$.

Figure 5 presents the fluorescence spectra recorded for the methanol-swollen network samples after being immersed for $24 \mathrm{~h}$ in methanol solutions containing various concentrations of $\mathrm{Fe}^{3+}$ or $\mathrm{Cu}^{2+}$, followed by extensive washing to remove any unbound metal ions. As seen in the fluorescence spectra, a quenching effect is observed in both cases. This is attributed to the presence of unpaired d electrons in transition metal ions that can effectively quench the anthryl chromophore. The quenching phenomenon is further increased upon increasing the metal ion concentration, which is in agreement with our previous studies. Buruiana and co-workers reported on the different quenching mechanisms that may occur in anthracene-containing systems [74]. These include excimer or exciplex formation, metal-p complex, electron transfer, and energy transfer. In the case of $\mathrm{Fe}^{3+}$ possessing unpaired $\mathrm{d}$ electrons, the quenching mechanism may involve an energy transfer process from the singlet excited-state anthracene chromophores to the low-lying half-filled $3 \mathrm{~d}$ orbitals of $\mathrm{Fe}^{3+}$ [75]. Besides the obvious decrease in the fluorescence intensity upon increasing the concentration of $\mathrm{Fe}^{3+}$, a blue shift is clearly observed in the fluorescence spectra (Figure 5a), whereas in the case of the $\mathrm{Cu}^{2+}$ the decrease in the fluorescence intensity is first preceded and then accompanied by a red shift (Figure 5b). According to Micheloni et al. [3], the coordination of metal ions may lead to an enhancement of the fluorescence emission (chelation-enhanced fluorescence effect, CHEF) or, as in the present study, 
to fluorescence quenching (chelation-enhancement quenching effect, CHEQ), whereas both effects may be coupled with a red or blue shift of the emission band.

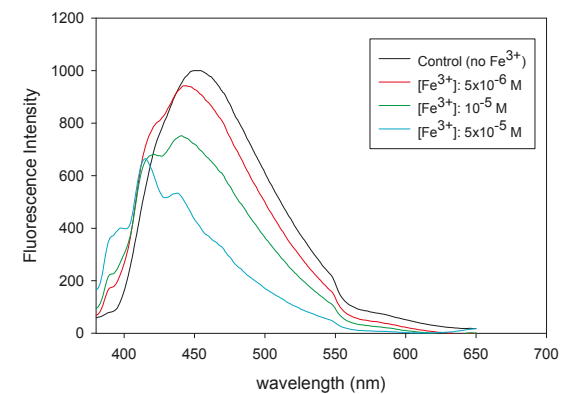

(a)

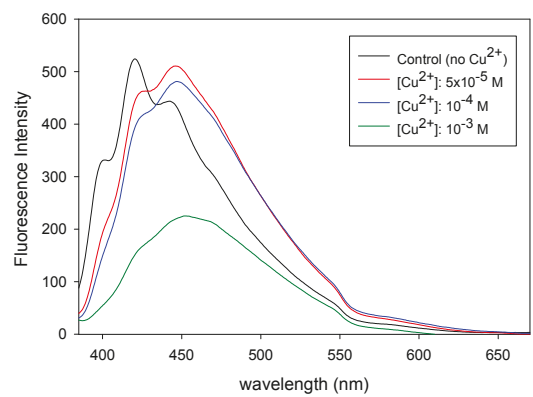

(b)

Figure 5. Fluorescence spectra of the methanol-swollen networks samples after being exposed to methanol solutions of various metal ion concentrations: (a) $\mathrm{Fe}^{3+}$ fluorescence monitoring; (b) $\mathrm{Cu}^{2+}$ monitoring.

According to the fluorescence spectroscopy data, in the case of the $\mathrm{Cu}^{2+}$ there is no obvious decrease in the fluorescence intensity until high $\mathrm{Cu}^{2+}$ concentrations $\left(10^{-3} \mathrm{M}\right)$ are reached, in contrast to the $\mathrm{Fe}^{3+}$ ions that act as effective quenchers for the anthracene fluorophores when present at much lower concentrations $\left(\sim 10^{-5} \mathrm{M}\right)$. These results are in line with the fluorescence microscopy images provided in Figure 4.

For further validation of the aforementioned results, control experiments were carried out in which 9-anthracenemethanol was used as the chemosensor in the monitoring of $\mathrm{Fe}^{3+}$ and $\mathrm{Cu}^{2+}$ in methanol. From the obtained data (Supplementary Materials, Figure S1, Table S1), the Stern-Volmer quenching plots were constructed (Figure 6), from which the Stern-Volmer quenching constants (Ksv) were determined from the Stern-Volmer equation, $I_{\mathrm{o}} / I=1+K_{\mathrm{sv}} \times\left(C_{\text {ion }}\right)$, to be $(0.33 \pm 0.04)$ and $(16.5 \pm 2.8) \mathrm{mM}^{-1}$ for the $\mathrm{Cu}^{2+}$ and $\mathrm{Fe}^{3+}$ quenchers, respectively. $I_{\mathrm{o}}$ and $I$ are the fluorescence intensities at $\sim 410 \mathrm{~nm}$ in the absence and presence of the metal ions, respectively, and $\left(C_{\text {ion }}\right)$ is the concentration of the metal ion quencher. The obtained results are in very good agreement with the fluorescence spectroscopy data obtained in the case where the networks were used as chemosensors, thus confirming the higher sensitivity of these systems towards the fluorescence detection of $\mathrm{Fe}^{3+}$ over $\mathrm{Cu}^{2+}$.

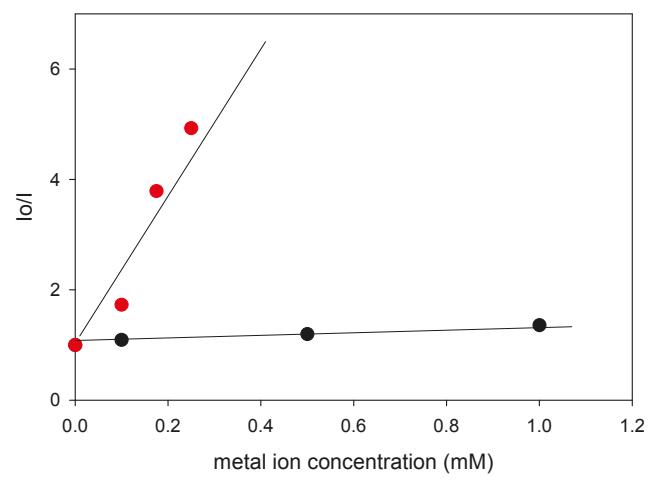

Figure 6. Stern-Volmer quenching plots of 9-anthracenemethanol in the presence of $\mathrm{Fe}^{3+}$ (red circles) and $\mathrm{Cu}^{2+}$ (black circles). 
The fact that in the control experiments using 9-anthracenemethanol as a chemosensor, which lacks metal ion-binding functionalities, no shift of the emission bands of the anthracene-containing fluorophore is observed in the presence of both metal ions (Supplementary Materials, Figure S1), strongly suggests that the semi-IPN network structure promotes the metal-ligand interactions via the metal-binding tertiary amino groups that are present on the pDMAEMA chains [58-63].

\subsection{Metal Ion Desorption-Network Regeneration}

The desorption of $\mathrm{Cu}^{2+}$ ions from the networks, and thus their regeneration, was accomplished upon immersing two different samples of the $\mathrm{Cu}^{2+}$-loaded networks (pre-equilibrated in $10^{-3}$ and $5 \times 10^{-3} \mathrm{M} \mathrm{Cu}^{2+}$ methanol solutions) in EDTA aqueous solutions. EDTA is a well-known chelating ligand that can bind onto various metal ions, including $\mathrm{Cu}^{2+}$, forming strong complexes. The metal ion desorption-regeneration process was qualitatively monitored in real time by fluorescence microscopy. As seen in the fluorescence images presented in Figure 7, an immediate increase in the fluorescence intensity of the networks was observed in both cases, qualitatively indicating desorption of the $\mathrm{Cu}^{2+}$ from the networks, thus resulting in the recovery of the networks' fluorescence efficiency.
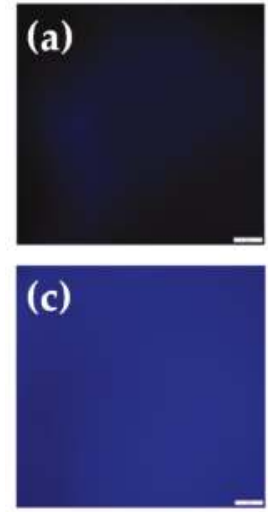
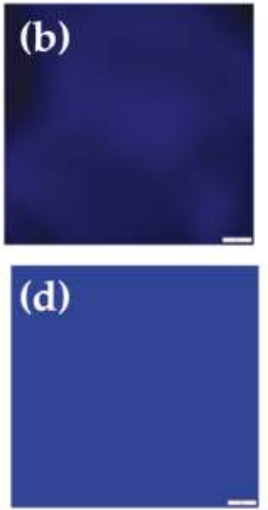

Figure 7. Fluorescence images analyzed using the CellSens software. Images $(\mathbf{a}, \mathbf{b})$ correspond to the $\mathrm{Cu}^{2+}$-loaded system equilibrated in $5 \times 10^{-3} \mathrm{M} \mathrm{Cu}^{2+}$ methanol solution prior to and after immersion in the ethylenediaminetetraacetic acid (EDTA) aqueous solution, respectively. Images (c,d) correspond to the $\mathrm{Cu}^{2+}$-loaded system equilibrated in $10^{-3} \mathrm{M} \mathrm{Cu}^{2+}$ methanol solution prior to and after immersion in the EDTA aqueous solution, respectively. The scale bar in Figure 7 is $100 \mu \mathrm{m}$.

\section{Conclusions}

We have demonstrated a simple and versatile methodology that leads to the generation of 3D fluorescent semi-IPN amphiphilic polymer networks with controlled architectures, deriving from the well-defined molecular characteristics of their linear precursors. Fluorescence spectroscopy and microscopy demonstrated that these materials behave as strong blue emitters under UV irradiation in the presence of only $5 \mathrm{wt} \%$ of the pAnMMA linear chains that are interlaced between BIEE-crosslinked pDMAEMA segments. These systems were further evaluated as sorbents for the uptake and fluorescence monitoring of transition metal ions $\left(\mathrm{Fe}^{3+}, \mathrm{Cu}^{2+}\right)$ in solution. The combination of the coordinating active part (pDMAEMA) with the fluorescent active component (pAnMMA) within a semi-IPN network architecture promotes a chelation-enhanced fluorescence quenching effect that is more pronounced in the case of $\mathrm{Fe}^{3+}$. Moreover, desorption of the $\mathrm{Cu}^{2+}$ from the networks could be realized upon immersion of the latter in an EDTA-containing solution, thus allowing their recyclability. 
Supplementary Materials: The following are available online at www.mdpi.com/2073-4360/8/12/411/s1, Table S1: Concentration of the metal ion quenchers $\left(\mathrm{Cu}^{2+}, \mathrm{Fe}^{3+}\right)$ and $I_{\mathrm{o}} / I$ data obtained by fluorescence spectroscopy when using 9-anthracenemethanol as a fluorophore; Figure S1: Fluorescence spectra of the 9-anthracenemethanol after being exposed to methanol solutions of various metal ion concentrations: $\mathrm{Cu}^{2+}$ fluorescence monitoring: left spectrum; $\mathrm{Fe}^{3+}$ monitoring: right spectrum.

Acknowledgments: This work was supported by the University of Cyprus. We are grateful to Maria Demetriou for the synthesis of the pAnMMA homopolymer used in the present study and to Triantafyllos Stylianopoulos (Cancer Biophysics Laboratory, Department of Mechanical and Manufacturing Engineering, University of Cyprus) for providing access to the fluorescence microscope.

Author Contributions: Theodora Krasia-Christoforou conceived and designed the experiments. Kyriakos Christodoulou and Mariliz Achilleos performed the synthesis of the networks. Kyriakos Christodoulou, Theodora Krasia-Christoforou and Epameinondas Leontidis performed the fluorescence spectroscopy studies and Christiana Polydorou carried out the fluorescence microscopy experiments. Epameinondas Leontidis contributed significantly to the interpretation of the obtained fluorescence spectroscopy data and designed the fluorescence quenching control experiments using 9-anthracenemethanol. Theodora Krasia-Christoforou wrote the paper.

Conflicts of Interest: The authors declare no conflict of interest.

\section{References}

1. Aron, A.T.; Ramos-Torres, K.M.; Cotruvo, J.A., Jr.; Chang, C.J. Recognition- and reactivity-based fluorescent probes for studying transition metal signaling in living systems. Acc. Chem. Res. 2015, 48, 2434-2442. [CrossRef] [PubMed]

2. Prodi, L.; Bolletta, F.; Montalti, M.; Zaccheroni, N. Luminescent chemosensors for transition metal ions. Coord. Chem. Rev. 2000, 205, 59-83. [CrossRef]

3. Formica, M.; Fusi, V.; Giorgi, L.; Micheloni, M. New fluorescent chemosensors for metal ions in solution. Coord. Chem. Rev. 2012, 256, 170-192. [CrossRef]

4. Fabbrizzi, L.; Licchelli, M.; Pallavicini, P.; Perotti, A.; Sacchi, D. An anthracene-based fluorescent sensor for transition metal ions. Angew. Chem. Int. Ed. 1994, 33, 1975-1977. [CrossRef]

5. Demetriou, M.; Krasia-Christoforou, T. Well-defined diblock copolymers possessing fluorescent and metal chelating functionalities as novel macromolecular sensors for amines and metal ions. J. Polym. Sci. Polym. Chem. 2012, 50, 52-60. [CrossRef]

6. Yang, J.; Wang, Z.; Li, Y.; Zhuang, Q.; Gu, J. Real-time monitoring of dissolved oxygen with inherent oxygen-sensitive centers in metal-organic frameworks. Chem. Mater. 2016, 28, 2652-2658. [CrossRef]

7. Zhao, S.-S.; Yang, J.; Liu, Y.-Y.; Ma, J.-F. Fluorescent aromatic tag-functionalized MOFs for highly selective sensing of metal ions and small organic molecules. Inorg. Chem. 2016, 55, 2261-2273. [CrossRef] [PubMed]

8. Wang, X.-P.; Han, L.-L.; Wang, Z.; Guo, L.-Y.; Sun, D. Microporous Cd(II) metal-organic framework as fluorescent sensor for nitroaromatic explosives at the sub-ppm level. J. Mol. Struct. 2016, 1107, 1-6. [CrossRef]

9. He, J.; Huang, J.; He, Y.; Cao, P.; Zeller, M.; Hunter, A.D.; Xu, Z. A boiling-water-stable, tunable white-emitting metal-organic framework from soft-imprint synthesis. Chem. Eur. J. 2016, 22, 1597-1601. [CrossRef] [PubMed]

10. Yang, L.; Li, X.; Qin, C.; Shao, K.-Z.; Su, Z.-M. A fluorescent sensor for highly selective sensing of nitro explosives and $\mathrm{Hg}(\mathrm{II})$ ions based on a 3D porous layer metal-organic framework. CrystEngComm 2016, 18, 4765-4771. [CrossRef]

11. Zhao, S.; Lv, X.-X.; Shi, L.-L.; Li, B.L.; Wu, B. An unusual (4,4)-connected 3D porous cadmium metal-organic framework as a luminescent sensor for detection of nitrobenzene. RSC Adv. 2016, 6, 56035-56041. [CrossRef]

12. Rachuri, Y.; Parmar, B.; Bisht, K.K.; Suresh, E. Mixed ligand two dimensional Cd(II)/Ni(II) metal organic frameworks containing dicarboxylate and tripodal $\mathrm{N}$-donor ligands: $\mathrm{Cd}(\mathrm{II}) \mathrm{MOF}$ is an efficient luminescent sensor for detection of picric acid in aqueous media. Dalton Trans. 2016, 45, 7881-7892. [CrossRef] [PubMed]

13. Gole, B.; Bar, A.K.; Mukherjee, P.S. Multicomponent assembly of fluorescent-tag functionalized ligands in metal-organic frameworks for sensing explosives. Chem. Eur. J. 2014, 20, 13321-13336. [CrossRef] [PubMed]

14. Yi, F.-Y.; Yang, W.; Sun, Z.-M. Highly selective acetone fluorescent sensors based on microporous Cd(II) metal-organic frameworks. J. Mater. Chem. 2012, 22, 23201-23209. [CrossRef]

15. He, J.; Yee, K.-K.; Xu, Z.; Zeller, M.; Hunter, A.-D.; Chui, S.S.-Y.; Che, C.-M. Thioether side chains improve the stability, fluorescence, and metal uptake of a metal-organic framework. Chem. Mater. 2011, 23, 2940-2947. [CrossRef] 
16. Liu, J.-J.; Guan, Y.-F.; Lin, M.-J.; Huang, C.-C.; Dai, W.-X. Luminescent coordination polymer with conjugated Lewis acid sites for the detection of organic amines. Cryst. Growth Des. 2015, 15, 5040-5046. [CrossRef]

17. Deng, J.; Ma, W.; Yu, P.; Mao, L. Colorimetric and fluorescent dual mode sensing of alcoholic strength in spirit samples with stimuli-responsive infinite coordination polymers. Anal. Chem. 2015, 87, 6958-6965. [CrossRef] [PubMed]

18. Lin, H.-Y.; Le, M.; Sui, F.-F.; Liu, G.-C. Two new 3D Zn(II)/Cu(II) coordination polymers based on $N, N^{\prime}$-Di(3-pyridyl)succinamide and two different aromatic dicarboxylates: Syntheses, crystal structures and properties. J. Chem. Crystallogr. 2015, 45, 94-102. [CrossRef]

19. Feng, X.; Li, R.; Wang, L.; Ng, S.W.; Qin, G.; Ma, L. A series of homonuclear lanthanide coordination polymers based on a fluorescent conjugated ligand: Syntheses, luminescence and sensor for pollutant chromate anion. CrystEngComm 2015, 17, 7878-7887. [CrossRef]

20. Xue, Z.; Sheng, T.; Wang, Y.; Hu, S.; Wen, Y.; Wang, Y.; Li, H.; Fu, R.; Wu, X. A series of d(10) coordination polymers constructed with a rigid tripodal imidazole ligand and varied polycarboxylates: Syntheses, structures and luminescence properties. CrystEngComm 2015, 17, 2004-2012. [CrossRef]

21. Dragan, E.S. Design and applications of interpenetrating polymer network hydrogels. A review. Chem. Eng. J. 2014, 243, 572-590. [CrossRef]

22. Kostanski, L.K.; Huang, R.; Filipe, C.D.M.; Ghosh, R. Interpenetrating Polymer Networks as a Route to Tunable Multi-responsive Biomaterials: Development of Novel Concepts. J. Biomater. Sci. Polym. Ed. 2009, 20, 271-297. [CrossRef] [PubMed]

23. Aminabhavi, T.M.; Nadagouda, M.N.; More, U.A.; Joshi, S.D.; Kulkarni, V.H.; Noolvi, M.N.; Kulkarni, P.V. Controlled release of therapeutics using interpenetrating polymeric networks. Expert Opin. Drug Deliv. 2015, 12, 669-688. [CrossRef] [PubMed]

24. Mespouille, L.; Hedrick, J.L.; Dubois, P. Expanding the role of chemistry to produce new amphiphilic polymer (co)networks. Soft Matter 2009, 5, 4878-4892. [CrossRef]

25. Siddhanta, S.K.; Gangopadhyay, R. Conducting polymer gel: Formation of a novel semi-IPN from polyaniline and crosslinked poly(2-acrylamido-2-methyl propanesulphonicacid). Polymer 2005, 46, 2933-3000. [CrossRef]

26. Bonillo, B.; Sprick, R.S.; Cooper, A.I. Tuning photophysical properties in conjugated microporous polymers by comonomer doping strategies. Chem. Mater. 2016, 28, 3469-3480. [CrossRef]

27. Geng, T.-M.; Zhu, H.; Song, W.; Zhu, F.; Wang, Y. Conjugated microporous polymer-based carbazole derivatives as fluorescence chemosensors for picronitric acid. J. Mater. Sci. 2016, 51, 4104-4114. [CrossRef]

28. Ji, X.; Yao, Y.; Li, J.; Yan, X.; Huang, F. A Supramolecular cross-linked conjugated polymer network for multiple fluorescent sensing. J. Am. Chem. Soc. 2013, 135, 74-77. [CrossRef] [PubMed]

29. Li, P.; Huang, L.; Lin, Y.; Shen, L.; Chen, Q.; Shi, W. Printable temperature-responsive hybrid hydrogels with photoluminescent carbon nanodots. Nanotechnology 2014, 25, 055603. [CrossRef] [PubMed]

30. Xu, L.; Wang, R.; Kelso, L.C.; Ying, Y.; Li, Y. A target-responsive and size-dependent hydrogel aptasensor embedded with QD fluorescent reporters for rapid detection of avian influenza virus H5N1. Sens. Actuators B Chem. 2016, 234, 98-108. [CrossRef]

31. Yan, J.-J.; Wang, H.; Zhou, Q.-H.; You, Y.-Z. Reversible and multisensitive quantum dot gels. Macromolecules 2011, 44, 4306-4312. [CrossRef]

32. Cellini, F.; Zhou, L.; Khapli, S.; Peterson, S.D.; Porfiri, M. Large deformations and fluorescence response of mechanochromic polyurethane sensors. Mech. Mater. 2016, 93, 145-162. [CrossRef]

33. Nguyen, T.H.; Venugopala, T.; Chen, S.; Sun, T.; Grattan, K.T.V.; Taylor, S.E.; Basheer, M.; Long, A.E. Fluorescence based fibre optic $\mathrm{pH}$ sensor for the $\mathrm{pH} 10-13$ range suitable for corrosion monitoring in concrete structures. Sens. Actuators B Chem. 2014, 191, 498-507. [CrossRef]

34. Li, Y.; Zhou, S. A simple method to fabricate fluorescent glucose sensor based on dye-complexed microgels. Sens. Actuators B Chem. 2013, 177, 792-799. [CrossRef]

35. Cajlakovic, M.; Lobnik, A.; Werner, T. Stability of new optical pH sensing material based on cross-linked poly(vinyl alcohol) copolymer. Anal. Chim. Acta 2002, 455, 207-213. [CrossRef]

36. Stock, N.; Biswas, S. Synthesis of metal-organic frameworks (MOFs): Routes to various MOF topologies, morphologies, and composites. Chem. Rev. 2012, 112, 933-969. [CrossRef] [PubMed]

37. Tuci, G.; Rossin, A.; Xu, X.; Ranocchiari, M.; van Bokhoven, J.A.; Luconi, L.; Manet, I.; Melucci, M.; Giambastiani, G. "Click" on MOFs: A versatile tool for the multimodal derivatization of N-3-decorated metal organic frameworks. Chem. Mater. 2013, 25, 2297-2308. [CrossRef] 
38. Oezdemir, E.; Thirion, D.; Yavuz, C.T. Covalent organic polymer framework with C-C bonds as a fluorescent probe for selective iron detection. RSC Adv. 2015, 5, 69010-69015. [CrossRef]

39. Jiang, H.-L.; Feng, D.; Liu, T.-F.; Li, J.-R.; Zhou, H.-C. Pore surface engineering with controlled loadings of functional groups via click chemistry in highly stable metal-organic frameworks. J. Am. Chem. Soc. 2012, 134, 14690-14693. [CrossRef] [PubMed]

40. Wang, Z.; Cohen, S.M. Postsynthetic modification of metal-organic frameworks. Chem. Soc. Rev. 2009, 38, 1315-1329. [CrossRef] [PubMed]

41. Resch-Genger, U.; Grabolle, M.; Cavaliere-Jaricot, S.; Nitschke, R.; Nann, T. Quantum dots versus organic dyes as fluorescent labels. Nat. Methods 2008, 5, 763-777. [CrossRef] [PubMed]

42. Lou, Q.; Qu, S.; Jing, P.; Ji, W.; Li, D.; Cao, J.; Zhang, H.; Liu, L.; Zhao, J.; Shen, D. Water-triggered luminescent "nano-bombs" based on supra-(carbon nanodots). Adv. Mater. 2015, 27, 1389-1394. [CrossRef] [PubMed]

43. Haraguchi, K.; Takehisa, T. Nanocomposite hydrogels: A unique organic-inorganic network structure with extraordinary mechanical, optical, and swelling/de-swelling properties. Adv. Mater. 2002, 14, 1120-1124. [CrossRef]

44. Blezer, R.; Lindhout, T.; Keszler, B.; Kennedy, J.P. Amphiphilic networks. VIII. Reduced in vitro thromboresistance of amphiphilic networks. Polym. Bull. 1995, 34, 101-107. [CrossRef]

45. Süvegh, K.; Domján, A.; Vankó, G.; Iván, B.; Vértes, A. Free volume and swelling dynamics of the poly [(2-dimethylamino) ethyl methacrylate]-l-polyisobutylene amphiphilic network by positron annihilation investigations. Macromolecules 1998, 31, 7770-7775. [CrossRef]

46. Hentze, H.-P.; Krämer, E.; Berton, B.; Förster, S.; Antonietti, M.; Dreja, M. Lyotropic mesophases of poly(ethylene oxide)- $b$-poly(butadiene) diblock copolymers and their cross-linking to generate ordered gels. Macromolecules 1999, 32, 5803-5809. [CrossRef]

47. Bromberg, L.; Temchenko, M.; Hatton, T.A. Dually Responsive microgels from polyether-modified poly(acrylic acid): Swelling and drug loading. Langmuir 2002, 18, 4944-4952. [CrossRef]

48. Simmons, M.R.; Yamasaki, E.N.; Patrickios, C.S. Cationic amphiphilic model networks: Synthesis by group transfer polymerization and characterization of the degree of swelling. Macromolecules 2000, 33, 3176-3179. [CrossRef]

49. Triftaridou, A.I.; Hadjiyannakou, S.C.; Vamvakaki, M.; Patrickios, C.S. Synthesis, characterization, and modeling of cationic amphiphilic model hydrogels: Effects of polymer composition and architecture. Macromolecules 2002, 35, 2506-2513. [CrossRef]

50. Vamvakaki, M.; Patrickios, C.S. Synthesis and characterization of electrolytic amphiphilic model networks based on cross-linked star polymers: Effect of star architecture. Chem. Mater. 2002, 14, 1630-1638. [CrossRef]

51. Gao, H.; Li, W.; Matyjaszewski, K. Synthesis of polyacrylate networks by ATRP: Parameters influencing experimental gel points. Macromolecules 2008, 41, 2335-2340. [CrossRef]

52. Rikkou, M.D.; Kolokasi, M.; Matyjaszewski, K.; Patrickios, C.S. End-linked amphiphilic polymer conetworks: Synthesis by sequential atom transfer radical polymerization and swelling characterization. J. Polym. Sci. Polym. Chem. 2010, 48, 1878-1886. [CrossRef]

53. Krasia-Christoforou, T.; Patrickios, C.S. Amphiphilic polymethacrylate model co-networks: Synthesis by RAFT radical polymerization and characterization of the swelling behavior. Macromolecules 2006, 39, 2467-2473. [CrossRef]

54. Achilleos, M.; Krasia-Christoforou, T.; Patrickios, C.S. Amphiphilic model conetworks based on combinations of methacrylate, acrylate, and styrenic units: Synthesis by RAFT radical polymerization and characterization of the swelling behavior. Macromolecules 2007, 40, 5575-5581. [CrossRef]

55. Wu, W.; Liu, J.; Cao, S.; Tan, H.; Li, J.; Xu, F.; Zhang, X. Drug release behaviors of a $\mathrm{pH}$ sensitive semi-interpenetrating polymer network hydrogel composed of poly(vinyl alcohol) and star poly[2-(dimethylamino)ethyl methacrylate]. Int. J. Pharm. 2011, 416, 104-109. [CrossRef] [PubMed]

56. Yao, F.; Xu, L.; Fu, G.-D.; Lin, B. Sliding-graft interpenetrating polymer networks from simultaneous "click chemistry" and atom transfer radical polymerization. Macromolecules 2010, 43, 9761-9770. [CrossRef]

57. Achilleos, M.; Mpekris, F.; Stylianopoulos, T.; Krasia-Christoforou, T. Structurally-defined semi-interpenetrating amphiphilic polymer networks with tunable and predictable mechanical response. RSC Adv. 2016, 6, 43278-43283. [CrossRef]

58. Shenmin Zhu, S.; Yang, N.; Zhang, D. Poly(N,N-dimethylaminoethyl methacrylate) modification of activated carbon for copper ions removal. Mater. Chem. Phys. 2009, 113, 784-789. 
59. Zhu, W.; Song, H.; Du, K.; Zeng, H.; Yao, S. Rapid removal of $\mathrm{Cr}(\mathrm{VI})$ ions from aqueous solutions by the macroporous poly $\left(N, N^{\prime}\right.$-dimethylamino ethyl methacrylate) hydrogels. J. Appl. Polym. Sci. 2013, 128, 2729-2735. [CrossRef]

60. Jiang, M.; Wang, J.; Li, L.; Pan, K.; Cao, B. Poly(N,N-dimethylaminoethyl methacrylate) modification of a regenerated cellulose membrane using ATRP method for copper(II) ion removal. RSC Adv. 2013, 3, 20625-20632. [CrossRef]

61. Zhao, L.; Sun, J.; Zhao, Y.; Xu, L.; Zhai, M. Removal of hazardous metal ions from wastewater by radiation synthesized silica-graft-dimethylaminoethyl methacrylate adsorbent. Chem. Eng. J. 2011, 170, 162-169. [CrossRef]

62. Tokuyama, H.; Ishihara, N. Temperature-swing adsorption of precious metal ions onto poly(2-(dimethylamino)ethyl methacrylate) gel. React. Funct. Polym. 2010, 70, 610-615. [CrossRef]

63. Kavakh, P.A.; Yilmaz, Z.; Sen, M. Investigation of heavy metal ion adsorption characteristics of poly(N,N-dimethylamino ethylmethacrylate) hydrogels. Sep. Sci. Technol. 2007, 42, 1245-1254. [CrossRef]

64. Cheng-Xiong, Y.; Hu-Bo, R.; Xiu-Ping, Y. Fluorescent metal-organic framework MIL-53(Al) for highly selective and sensitive detection of $\mathrm{Fe}^{3+}$ in aqueous solution. Anal. Chem. 2013, 85, 7441-7446.

65. Krämer, R. Fluorescent chemosensors for $\mathrm{Cu}^{2+}$ Ions: Fast, selective, and highly sensitive. Angezv. Chem. 1998, 37, 772-773. [CrossRef]

66. Ferreira de Deus, J.; Andrade, M.L.; Atvars, T.D.Z.; Akcelrud, L. Photo and electroluminescence studies of poly(methyl methacrylate-co-9-anthryl methyl methacrylate). Chem. Phys. 2004, 297, 177-186. [CrossRef]

67. Li, Y.; Ryan, A.J.; Armes, S.P. Synthesis of well-defined branched copolymers by quaternization of near-monodisperse homopolymers. Macromolecules 2008, 41, 5577-5581. [CrossRef]

68. Byron, C.M.; Werner, T.C. Experiments in synchronous fluorescence spectroscopy for the undergraduate instrumental chemistry course. J. Chem. Educ. 1991, 68, 433-436. [CrossRef]

69. Visscher, A.; Bachmann, S.; Schnegelsberg, C.; Teuteberg, T.; Mata, R.A.; Stalke, D. Highly selective and sensitive fluorescence detection of $\mathrm{Zn}^{2+}$ and $\mathrm{Cd}^{2+}$ ions by using an acridine sensor. Dalton Trans. 2016, 45, 5689-5699. [CrossRef] [PubMed]

70. Gupta, V.K. A highly selective fluorescence and electrochemical sensor for $\mathrm{Ni}(\mathrm{II})$ ions based on 4-(4-phenylthiazole-2-ylimino)pent-2-en-2-ol. Int. J. Electrochem. Sci. 2015, 10, 7854-7865.

71. Dereka, B.; Svechkarev, D.; Doroshenko, A.O. Facile ultrasensitive monitoring of mercury ions in water by fluorescent ratiometric detection. Cent. Eur. J. Chem. 2013, 11, 584-593. [CrossRef]

72. Yu, C.; Zhang, J.; Li, J.; Liu, P.; Wei, P.; Chen, L. Fluorescent probe for copper(II) ion based on a rhodamine spirolactame derivative, and its application to fluorescent imaging in living cells. Microchim. Acta 2011, 174, 247-255. [CrossRef]

73. Raje, V.P.; Morgado, P.I.; Ribeiro, M.P.; Correia, I.J.; Bonifácio, V.D.B.; Branco, P.S.; Aguiar-Ricardo, A. Dual on-off and off-on switchable oligoaziridine biosensor. Biosens. Bioelectron. 2013, 39, 64-69. [CrossRef] [PubMed]

74. Buruiana, E.C.; Olaru, M.; Simionescu, B.C. Photochemical aspects in anthracene-containing cationic polyurethanes. Eur. Polym. J. 2007, 43, 1359-1371. [CrossRef]

75. Weizman, H.; Ardon, O.; Mester, B.; Libman, J.; Oren Dwir, O.; Hadar, Y.; Chen, Y.; Shanzer, A. Fluorescently-labeled ferrichrome analogs as probes for receptor-mediated, microbial iron uptake. J. Am. Chem. Soc. 1996, 118, 12368-12375. [CrossRef]

(c) 2016 by the authors. Licensee MDPI, Basel, Switzerland. This article is an open access article distributed under the terms and conditions of the Creative Commons Attribution (CC BY) license (http:/ / creativecommons.org/licenses/by/4.0/). 
Article

\title{
Thixotropic Supramolecular Pectin-Poly(Ethylene Glycol) Methacrylate (PEGMA) Hydrogels
}

\author{
Siew Yin Chan ${ }^{1,2}$, Wee Sim Choo ${ }^{1, *}$, David James Young ${ }^{1,2,3, *}$ and Xian Jun Loh ${ }^{2,4,5, *}$ \\ 1 School of Science, Monash University Malaysia, Subang Jaya 47500, Malaysia; siew.chan@monash.edu \\ 2 Institute of Materials Research and Engineering (IMRE), A*STAR (Agency for Science, \\ Technology and Research), Singapore 138634, Singapore \\ 3 Faculty of Science, Health, Education and Engineering, University of the Sunshine Coast, \\ Sunshine Coast, QLD 4558, Australia \\ 4 Department of Materials Science and Engineering, National University of Singapore, \\ Singapore 117576, Singapore \\ 5 Singapore Eye Research Institute, Singapore 168751, Singapore \\ * Correspondence: choo.wee.sim@monash.edu (W.S.C.); \\ dyoung1@usc.edu.au (D.J.Y.); lohxj@imre.a-star.edu.sg (X.J.L.); \\ Tel.: +60-3-5514-6114 (W.S.C.); +61-7-5456-3448 (D.J.Y.); +65-6416-8932 (X.J.L.)
}

Academic Editor: Alexander Böker

Received: 2 October 2016; Accepted: 10 November 2016; Published: 18 November 2016

\begin{abstract}
Pectin is an anionic, water-soluble polymer predominantly consisting of covalently 1,4-linked $\alpha$-D-galacturonic acid units. This naturally occurring, renewable and biodegradable polymer is underutilized in polymer science due to its insolubility in organic solvents, which renders conventional polymerization methods impractical. To circumvent this problem, cerium-initiated radical polymerization was utilized to graft methoxy-poly(ethylene glycol) methacrylate (mPEGMA) onto pectin in water. The copolymers were characterized by ${ }^{1} \mathrm{H}$ nuclear magnetic resonance (NMR), Fourier transform infrared (FTIR) spectroscopy and thermogravimetric analysis (TGA), and used in the formation of supramolecular hydrogels through the addition of $\alpha$-cyclodextrin $(\alpha-C D)$ to induce crosslinking. These hydrogels possessed thixotropic properties; shear-thinning to liquid upon agitation but settling into gels at rest. In contrast to most of the other hydrogels produced through the use of poly(ethylene glycol) (PEG)-grafted polymers, the pectin-PEGMA / $\alpha$-CD hydrogels were unaffected by temperature changes.
\end{abstract}

Keywords: pectin; poly(ethylene glycol) methacrylate; cerium; $\alpha$-cyclodextrin; supramolecular hydrogel

\section{Introduction}

Pectins are complex carbohydrates derived from dicotyledonous and some monocotyledonous plants [1]. Commercially, pectins are produced from food industry waste [2]. They are mainly extracted with hot dilute mineral acid from citrus peel, apple pomace and to a smaller extent, sugar beet pulp [3,4]. Pectin is a safe food additive with no limit on acceptable daily intake. It is non-toxic, biodegradable and biocompatible [5,6]. Its applications are diverse spanning the food, pharmaceutical, cosmetic and polymer industries [7-9]. Pectins are primarily utilized as emulsifiers, gelling agents, glazing agents, stabilizers or thickeners [10].

This family of polysaccharides consists of galacturonic acid (GalA) units covalently-linked by $\alpha-(1 \rightarrow 4)$ glycosidic bonds and are classified according to the degree of methylation (DM). DM is defined as the molar ratio of methyl-esters present relative to the total moles of GalA units. It is the major parameter affecting gelling [11]. Pectins are classified as either high methoxy pectin (HMP) with a $\mathrm{DM}>50 \%$ or low methoxy pectin (LMP) with a $\mathrm{DM}<50 \%$. HMP gels in high co-solute concentration 
with acid through a combination of hydrophobic forces and hydrogen bonding [12]. LMP gels in the presence of divalent metal cations such as calcium over a broad range of $\mathrm{pH}$ and the gels are formed through ionic cross-linking between free carboxylate groups in an arrangement known as the egg-box model [13].

We have an interest in grafting pectin with synthetic polymers to improve its processability in organic solvents and to impart biocompatibility and biodegradability to the resulting copolymer for use in biomedical applications. Herein, we describe pectin-poly(ethylene glycol) methacrylate (PEGMA) copolymer and subsequently generated supramolecular hydrogels by threading the poly(ethylene glycol) (PEG) tendrils with $\alpha$-cyclodextrin $(\alpha-\mathrm{CD})$ units. Harada et al. [14] first reported supramolecular hydrogel formed by threading $\alpha$-cyclodextrin toroids onto PEG of high molecular weight. This PEG-graft forms physical cross-linking points via the aggregation of the inclusion complexes, yielding supramolecular hydrogels [15-19].

Pectin is a hydrophilic polymer and insoluble in all organic solvents. Most polymerization methods require organic solvents, making it a challenge to graft pectin with synthetic polymers. We have for the first time utilized a redox polymerization reaction in water to graft PEGMA onto pectin and have thereby generated pectin supramolecular gels with unique rheology.

\section{Materials and Methods}

Ammonium cerium (IV) sulfate dihydrate, apple pectin and sodium nitrate were purchased from Sigma-Aldrich, Singapore, Singapore. Methoxy-poly(ethylene glycol) methacrylate (mPEGMA) with Mn of 10,000 g. $\mathrm{mol}^{-1}$ was purchased from Sinopeg, Xiamen, China. $\alpha$-Cyclodextrin $(\alpha-C D)$ was purchased from TCI, Kawaguchi, Japan. All reagent and solvents were used as received.

\subsection{Synthesis of Pectin-PEGMA Copolymer, P-10K}

Ammonium cerium (IV) sulfate dihydrate $(0.005 \mathrm{M}$, based on the final volume of solution) was added into a pectin solution ( $1 \% w / v$ in water). The solution was stirred at room temperature for $2 \mathrm{~h}$. mPEGMA $(1 \% w / v)$ was then added. The reaction mixture was stirred at room temperature for $48 \mathrm{~h}$. Chloroform was added and the resulting mixture was left to settle. Pectin-PEGMA was obtained in $61.5 \%$ yield by precipitating the white emulsion layer in hexane followed by drying under vacuum at $40{ }^{\circ} \mathrm{C}$. The mechanism of cerium-initiated radical polymerization is shown in Figure 1.

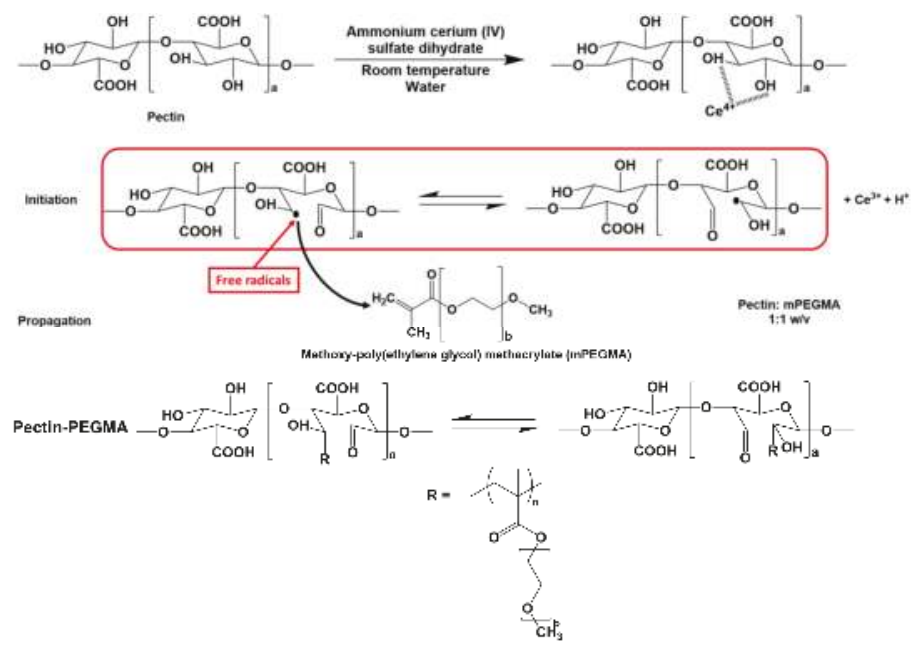

Figure 1. Mechanism of cerium-initiated radical polymerization. 


\subsection{Polymer Characterisation}

${ }^{1} \mathrm{H}$ Nuclear magnetic resonance (NMR) spectra were recorded on a JEOL $500 \mathrm{MHz} \mathrm{NMR}$ spectrometer (JEOL, Tokyo, Japan) at room temperature. The ${ }^{1} \mathrm{H}$ NMR measurements were carried out with an acquisition time of $4.37 \mathrm{~s}$, a pulse repetition time of $9.37 \mathrm{~s}$ and a $90^{\circ}$ pulse width. Measurements were done with 16 scans and chemical shifts were referred to the solvent peak $(\delta=4.79$ ppm for deuterium oxide, $\mathrm{D}_{2} \mathrm{O}$ ).

Fourier transform infrared (FTIR) spectra of the pellet samples were recorded on a Perkin Elmer Spectrum 2000 FTIR spectrometer (Perkin Elmer, Waltham, MA, USA); 64 scans were signal-averaged with a resolution of $4 \mathrm{~cm}^{-1}$ at room temperature. Pellets were prepared by coating the samples with potassium bromide.

\subsection{Thermal Analysis}

Thermogravimetric analysis (TGA) was performed on a TA Instruments TGA Q500 (TA Instruments, New Castle, DE, USA). Samples were heated from room temperature to $900{ }^{\circ} \mathrm{C}$ at a rate of $20^{\circ} \mathrm{C} \cdot \mathrm{min}^{-1}$ under continuous nitrogen purge with a flow rate of $40 \mathrm{~mL} \cdot \mathrm{min}^{-1}$.

\subsection{Preparation of Pectin-PEGMA/ $\alpha-C D$ Hydrogels}

Solutions of $\alpha$-CD in water were added into pectin-PEGMA solutions of different compositions. The resultant mixtures were sonicated and left to stand at room temperature. Gel formation was observed at $24 \mathrm{~h}$ (Figure 2). The sol-gel transition for P-10K was determined by plotting $\alpha$-CD concentration versus polymer concentration over the polymer to $\alpha$-CD solution composition range of $1 \%-10 \% w / v$, permitting determination of the critical gelation concentration.

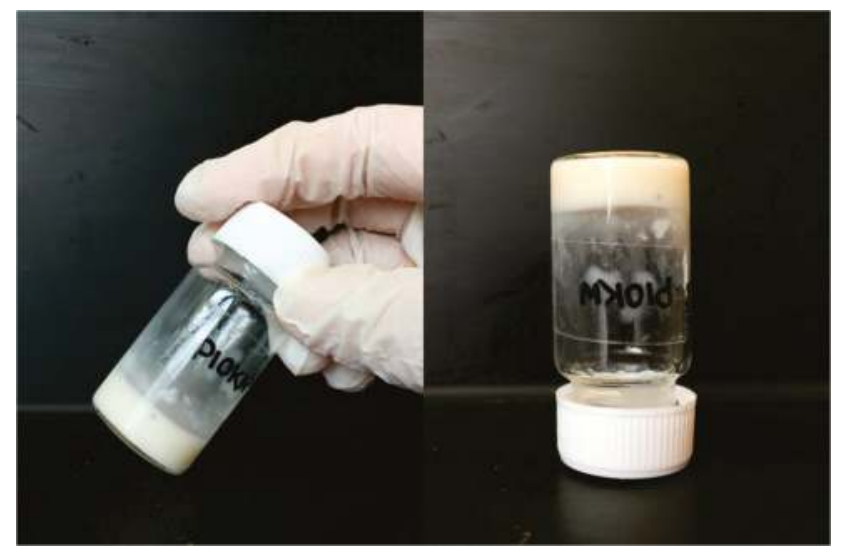

Figure 2. The preparation of pectin-PEGMA/ $\alpha$-CD gels

\subsection{Rheology Studies}

The rheological behavior of pectin-PEGMA/ $\alpha$-CD gels was investigated using a Discovery DHR-3 hybrid rheometer (TA Instruments, New Castle, DE, USA) with flat plate geometries (diameters of 20 and $40 \mathrm{~mm}$ ) in steady and dynamic modes. All the tests were performed using a flat plate geometry with diameter of $40 \mathrm{~mm}$ unless otherwise stated. Amplitude sweeps were performed under oscillatory shear at strain of $0.01 \%-100 \%$ to ensure that subsequent data were collected in the linear viscoelastic region (LVR). Frequency sweeps were then performed in the range of $0.01-50 \mathrm{~Hz}$ under oscillatory shear at a strain of $0.05 \%$. Reversibility of the hydrogels was determined by amplitude sweeps at 2 points ( $0.05 \%$ and $25 \%$ strain), 5 and $2.5 \mathrm{~min}$, respectively, at each strain point, for 3 cycles. All tests 
were performed at $25^{\circ} \mathrm{C}$ (room temperature) and $37^{\circ} \mathrm{C}$ to mimic body temperature. Temperature sweeps were performed using a flat plate geometry with diameter of $20 \mathrm{~mm}$ at a strain of $0.5 \%$ and frequency of $1 \mathrm{~Hz}$ in the temperature range of $10-40^{\circ} \mathrm{C}$, with a ramp rate of $5^{\circ} \mathrm{C} \cdot \mathrm{min}^{-1}$.

\section{Results}

\subsection{Synthesis and Characterization of Pectin-PEGMA Copolymer, P-10K}

Pectin-PEGMA copolymer (P-10K) was prepared by radical polymerization in distilled water using ammonium cerium (IV) sulfate dihydrate as a redox initiator. Chloroform was added to extract excess PEGMA. Three layers formed in settling; a cloudy brown solution, a white middle layer emulsion and at the bottom a transparent chloroform containing the unreacted mPEGMA.

${ }^{1} \mathrm{H}$ NMR spectroscopy in $\mathrm{D}_{2} \mathrm{O}$ was performed on the middle emulsion layer, but proved inconclusive (Figure S1). FTIR, however, contained absorption characteristics of pectin and mPEGMA (10KPEGMA). Pectin exhibits a broad peak at around $3450 \mathrm{~cm}^{-1}$, arising from hydroxyl group stretching $(\mathrm{O}-\mathrm{H})$ (Figure 3) [20]. Absorptions at 1750 and $1630 \mathrm{~cm}^{-1}$ were assigned to the carboxylic acid and ester groups ( $\mathrm{C}=\mathrm{O}$ stretches) of the pectin polymers [21]. 10KPEGMA displays a broad peak around $3450 \mathrm{~cm}^{-1}$ but not as intensive as for pectin. The FTIR spectrum of 10KPEGMA also contained distinctive $\mathrm{C}-\mathrm{H}$ stretches at $2875 \mathrm{~cm}^{-1}$. $\mathrm{CH}_{2}$ (bending) stretches at $1450 \mathrm{~cm}^{-1}, \mathrm{CH}_{3}$ (bending) stretches at $1350 \mathrm{~cm}^{-1}$ and $\mathrm{C}-\mathrm{O}$ stretches at $1110 \mathrm{~cm}^{-1}$ were also observed. The FTIR spectrum obtained for 10KPEGMA matched those reported by Wang et al. [22] and Bagheri et al. [23].

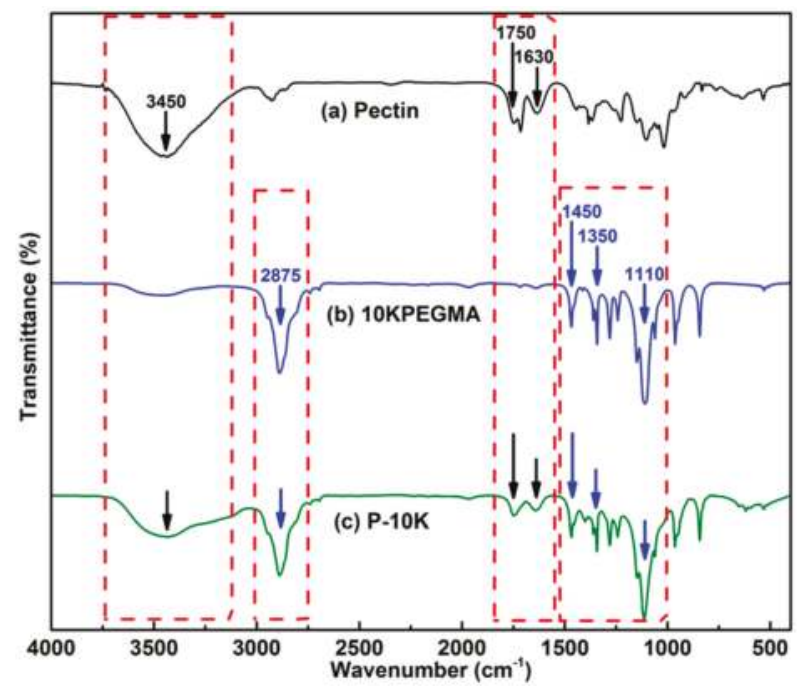

Figure 3. FTIR spectra of the precursors (pectin and 10KPEGMA) and of copolymer P-10K.

\subsection{Thermal Analysis}

Thermal stabilities of the precursors and copolymer were studied by TGA. The degradation temperatures were determined from the peak of derivative weight curves and are summarized in Table 1. There were two degradation temperatures recorded for pectin; 86.33 and $253.33{ }^{\circ} \mathrm{C}$ (Figure 4). The first degradation temperature corresponds to the loss of water. 10KPEGMA exhibited a degradation temperature of $413.53{ }^{\circ} \mathrm{C}$. TGA of $\mathrm{P}-10 \mathrm{~K}$ revealed three degradation temperatures, corresponding to those for pectin and mPEGMA. The amount of pectin in P-10K was calculated to be around $17 \%$ (Table 1). 
Table 1. Decomposition temperatures $\left(T_{\mathrm{d}}\right)$ of the precursors (pectin and 10KPEGMA) and copolymer P-10K.

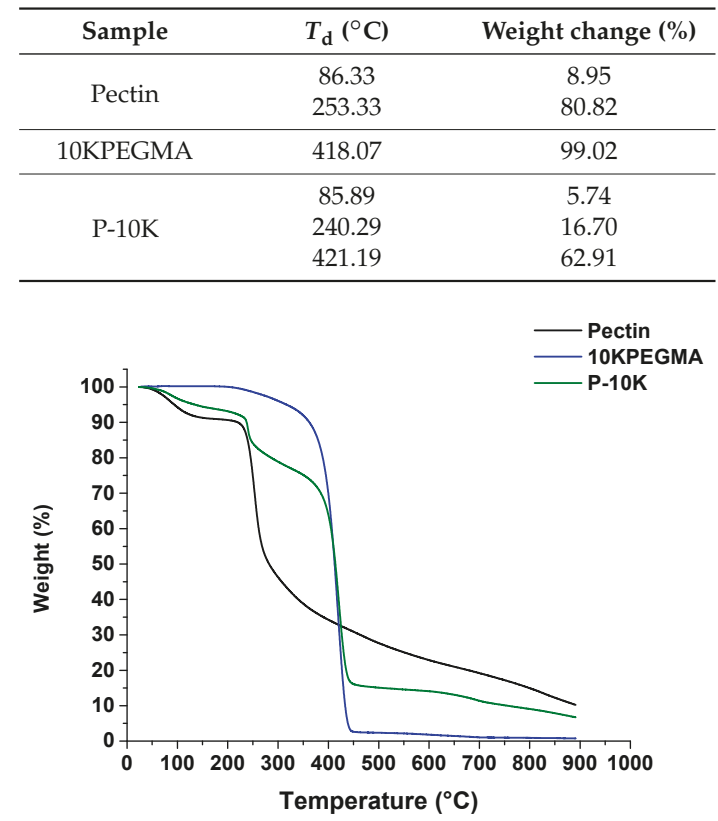

Figure 4. TGA curves of the precursors (pectin and 10KPEGMA) and copolymer P-10K.

\subsection{Critical Gelation Concentration Determination}

Aqueous solutions of $\alpha-C D$ were added to P-10K solutions and the mixtures became gradually opaque. The mixtures turned white and formed gels after a time period. The effects of the polymer and $\alpha$-CD concentrations on gelling behavior were studied by mixing a range of polymer and $\alpha-C D$ compositions $(1 \%-10 \% w / v)$. Hydrogel could be formed at a low polymer concentration of $1 \%(w / v)$ (Figure 5). The copolymer gelled at an optimum ratio of $1 \%(w / v)$ polymer and $5 \%(w / v) \alpha-C D$. Ye et al. have reported that higher cyclodextrin concentrations increase the gel strength [24].

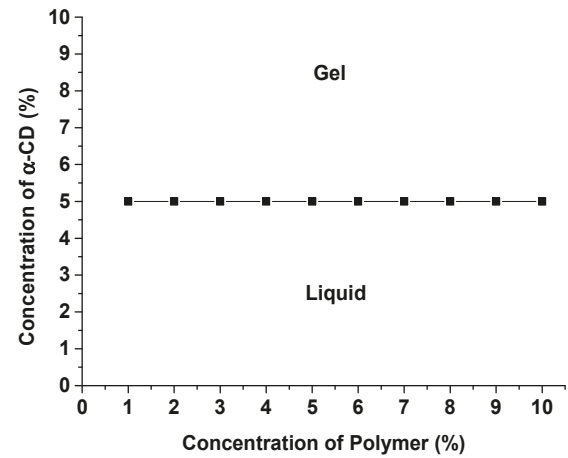

Figure 5. Sol-gel transition graph for different compositions of $\mathrm{P}-10 \mathrm{~K} / \alpha-\mathrm{CD}$. 


\subsection{Rheology of Pectin-PEGMA/ $\alpha$-CD Hydrogel}

The rheology of hydrogels prepared with $10 \% \mathrm{P}-10 \mathrm{~K}$ and $10 \% \alpha$-CD was investigated. Amplitude sweeps to measure shear strain were in the range of $0.01 \%-100 \%$ at temperatures of $25{ }^{\circ} \mathrm{C}$ (room temperature) and $37^{\circ} \mathrm{C}$ (body temperature). The hydrogels proved to be highly structured, true gels with storage moduli $\left(G^{\prime}\right)$ greater then loss moduli $\left(G^{\prime \prime}\right)$ at low shear strain (Figure 6). As the shear strain increased, $G^{\prime}$ began to decrease at a rate faster than that of $G^{\prime \prime}$. As the oscillation strain increased from $0.01 \%$ to $100 \%$, the hydrogels changed from a gel $\left(G^{\prime}>G^{\prime \prime}\right)$ to a liquid $\left(G^{\prime}<G^{\prime \prime}\right)$. The critical strain of the hydrogel was around $7 \%-11 \%$ for P-10K. At a shear strain greater than the critical strain, $G^{\prime}$ dropped below $G^{\prime \prime}$, indicating that the gel network structures had been disrupted by shearing. The linear viscoelastic region (LVR) of the hydrogels was $0.01 \%$ to $1 \%$ strain [25].

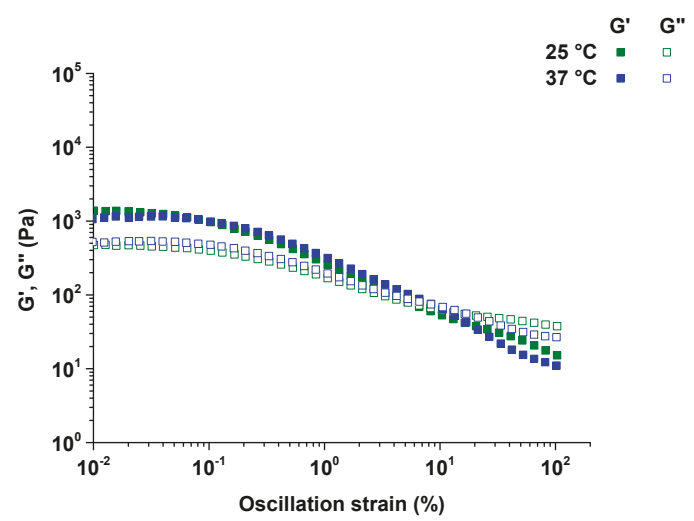

Figure 6. Amplitude sweeps performed from $0.01 \%$ to $100 \%$ of oscillation strain at 25 and $37{ }^{\circ} \mathrm{C}$ on hydrogels prepared with $10 \% \mathrm{P}-10 \mathrm{~K}$ and $10 \% \alpha-\mathrm{CD}$.

Frequency sweeps were performed on the hydrogels from $0.01-50 \mathrm{~Hz}$ at $0.05 \%$ strain at 25 and $37^{\circ} \mathrm{C}$. $G^{\prime}$ were higher than $G^{\prime \prime}$ and both the moduli were dependent on frequency at both 25 and $37^{\circ} \mathrm{C}$ (Figure 7). Frequency sweeps provide information on the effect of colloidal forces or the interaction among particles [26]. As frequency increased, there appeared to be no interaction between particles, presumably because of sufficient separation to eliminate interactions and/or collisions.

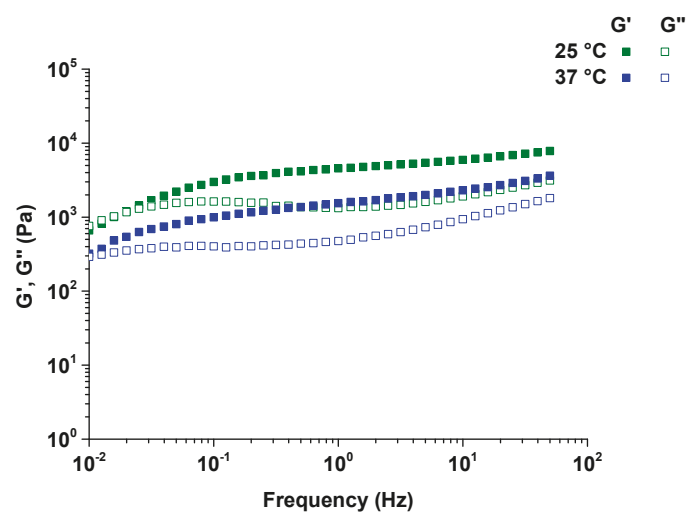

Figure 7. Frequency sweeps performed from 0.01 to $50 \mathrm{~Hz}$ of oscillation strain at 25 and $37^{\circ} \mathrm{C}$ on hydrogels prepared with $10 \% \mathrm{P}-10 \mathrm{~K}$ and $10 \% \alpha$-CD. 


\subsection{Thixotropic Properties of Pectin-PEGMA/ $\alpha-C D$ Hydrogels}

Instantaneously varying strain at $0.05 \%$ and $25 \%$ in amplitude sweeps for three cycles indicated that P-10K/ $\alpha-C D$ hydrogels recovered their original gel structure (Figure 8) [27]. G' was higher than $G^{\prime \prime}$ during the first $5 \mathrm{~min}$ of $0.05 \%$ strain and the gel was immediately sheared into a liquid where $G^{\prime}<G^{\prime \prime}$ during the $2.5 \mathrm{~min}$ of $25 \%$ strain. The liquid then reverted back to gel $\left(G^{\prime}>G^{\prime \prime}\right)$ when the strain was reduced to $0.05 \%$. However, the hydrogel required some recovery time to revert back to the original gel form. The internal network structure could be broken down by shearing, and required time to rebuild, as shown by the coincides of $G^{\prime}$ and $G^{\prime \prime}$ at each $0.05 \%$ strain point after being sheared at $25 \%$ strain. This shear-recovery phenomenon was repeatable for all the hydrogels at a minimum of three cycles, proving the thixotropy of P-10K/ $\alpha$-CD hydrogels.

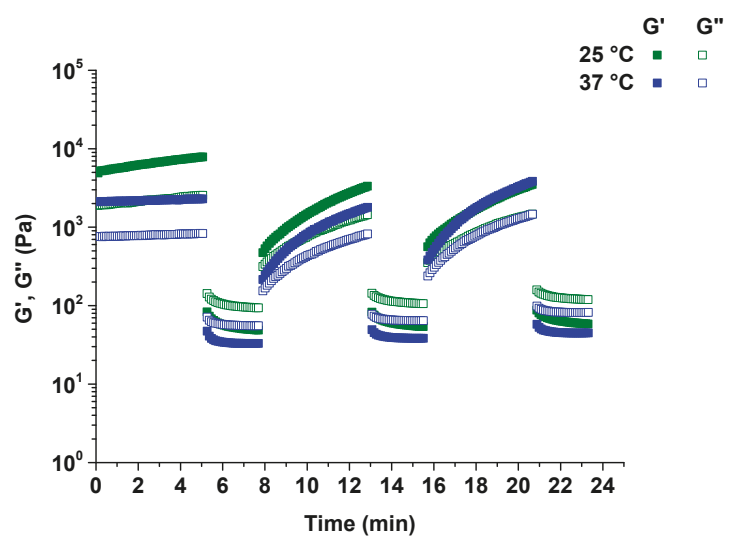

Figure 8. Amplitude sweeps at $0.05 \%$ and $25 \%$ strains performed instantaneously for three cycles at 25 and $37^{\circ} \mathrm{C}$ on hydrogels prepared with $10 \%$ P-10K and $10 \% \alpha$-CD.

\subsection{The Effect of Temperature on Pectin-PEGMA/ $\alpha-C D$ Hydrogels}

Temperature sweeps were performed to probe the temperature responsiveness of $\mathrm{P}-10 \mathrm{~K} / \alpha-\mathrm{CD}$ hydrogels (Figure 9) and demonstrated that these gels were not affected by temperature from 10 to $40{ }^{\circ} \mathrm{C}$. $G^{\prime}$ was higher than $G^{\prime \prime}$ and the moduli remained almost constant over this range.

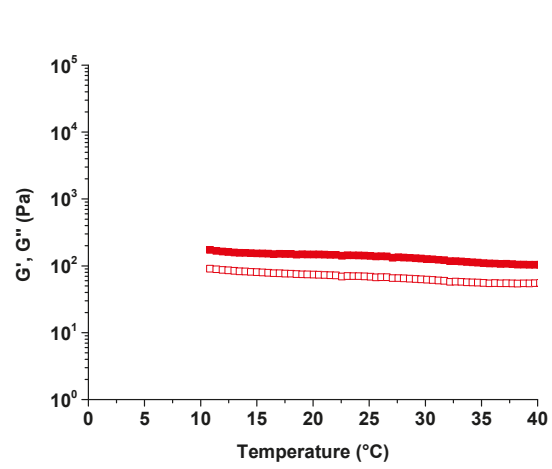

Figure 9. Temperature sweep performed from 10 to $40{ }^{\circ} \mathrm{C}$ on hydrogel prepared with $10 \% \mathrm{P}-10 \mathrm{~K}$ and $10 \% \alpha$-CD. 


\section{Discussion}

Pectin-PEGMA copolymer was successfully synthesized through cerium-initiated radical polymerization, as evidenced by FTIR spectroscopy (Figure 3) and TGA (Figure 4). The latter, in particular, was diagnostic, indicating the presence of two components in P-10K and a shift in the degradation temperature of the copolymer relative to its component precursors. Based on the weight change determined from these TGA curves (Table 1), it can be concluded there was $17 \%$ pectin in P-10K (Table 1). The FTIR spectra of this block copolymer supported the presence of pectin as a minority component (Figure 3).

Pectin-PEGMA copolymer was able to form gels with $\alpha$-CD (Figure 5). The $\alpha$-CD threaded onto PEG tendrils to form stacked inclusion complexes (Figure 10) $[28,29]$ that interact with each other to aggregate via hydrogen bonding [30]. These polypseudorotaxanes can inter- or intra-crosslink with each other while some chains of stacked inclusion complexes may remain separated (Figure 11). A control experiment involving mPEGMA alone turned opaque and eventually white when $\alpha$-CD was added, but no gel was formed (Table 2). Inclusion complexes still formed between $\alpha-C D$ and PEG chains. However, the columns of threaded PEG chains remained free with no gelation [31]. P-10K required $5 \% \alpha$-CD to gel (Figure 5). Upon addition of $10 \% \alpha$-CD into $10 \% 10 \mathrm{KPEGMA}$ and $10 \% \mathrm{P}-10 \mathrm{~K}$ solutions, respectively, the co-polymer sample gelled, but not mPEGMA (Figure 12).

The response of pectin-PEGMA / $\alpha$-CD hydrogel to shear strain and its dependence on frequency demonstrated shear-thinning. The higher the strain percentage was, the more liquid-like the hydrogel became. The time oscillation test further demonstrated that the hydrogel was thixotropic. Thixotropic materials provide a gel consistency when at rest and flow when shear is introduced (Figure 13). Pectin-PEGMA/ $\alpha$-CD hydrogel was sheared beyond critical strain, but reverted back to gel on resting. Most PEG-grafted polymers are temperature-sensitive, with the network structure disrupted by an increase in temperature $[15,19]$. High temperature may disrupt the non-covalent interactions in supramolecular hydrogels, causing $\alpha$-CD to de-thread from PEG chains [15]. The physical cross-linked network will then collapse. This effect was not observed for pectin-PEGMA/ $\alpha$-CD hydrogel which maintained its gel consistency from 10 to $40{ }^{\circ} \mathrm{C}$. We hypothesize that pectin may confer thermal stability to the pectin-PEGMA / $\alpha$-CD hydrogels. In support of this proposition, it has been reported that heat treatment of milk does not affect the ability of pectin to stabilize particles such as casein and denatured whey complex [32].

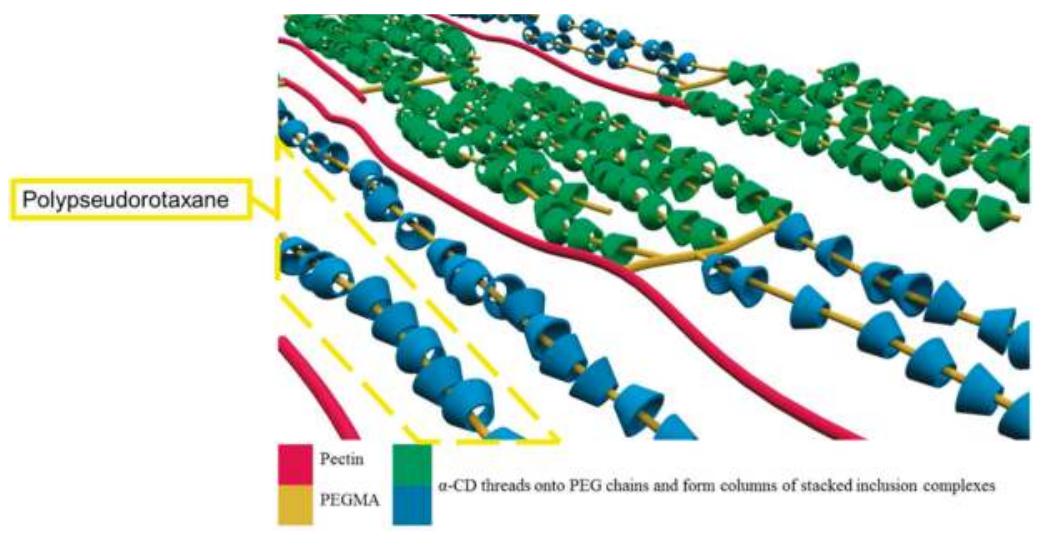

Figure 10. Threading of $\alpha$-CD onto PEG chains to form columns of stacked inclusion complexes. 


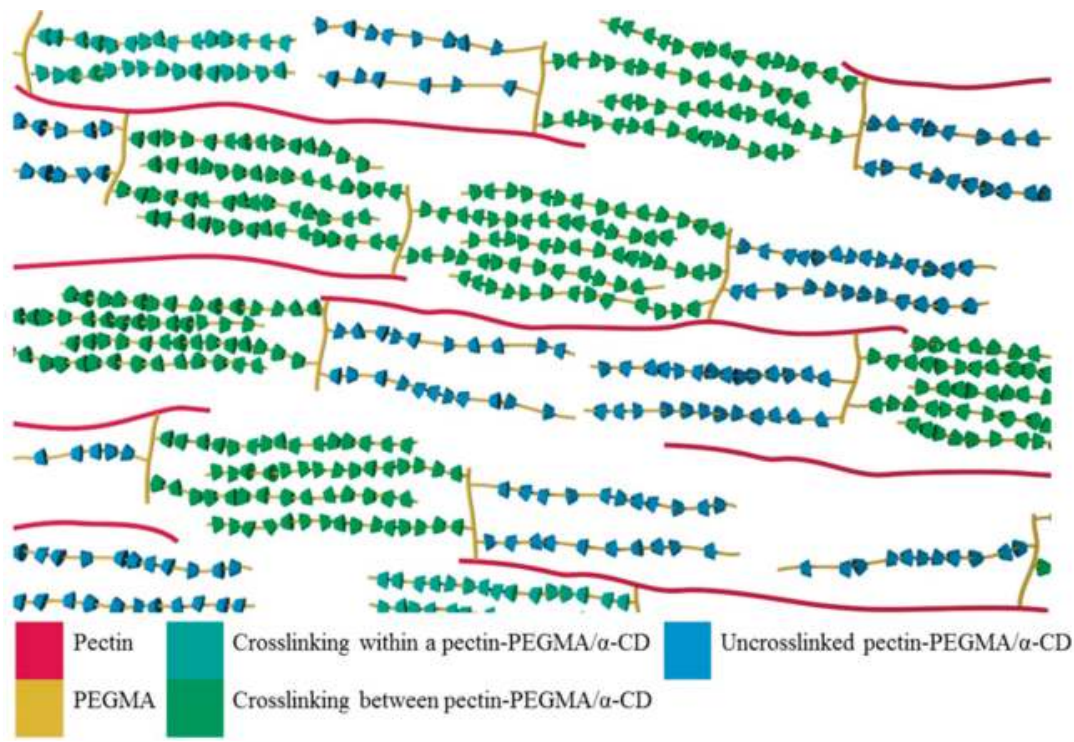

Figure 11. Polypseudorotaxanes inter- or intra-crosslink with each other via hydrogen bonding.

Table 2. Ten percent 10KPEGMA and 10\% P-10K solutions with $10 \% \alpha$-CD.

\begin{tabular}{cc}
\hline Sample & Gel \\
\hline 10KPEGMA & $x$ \\
P-10K & $\checkmark$
\end{tabular}

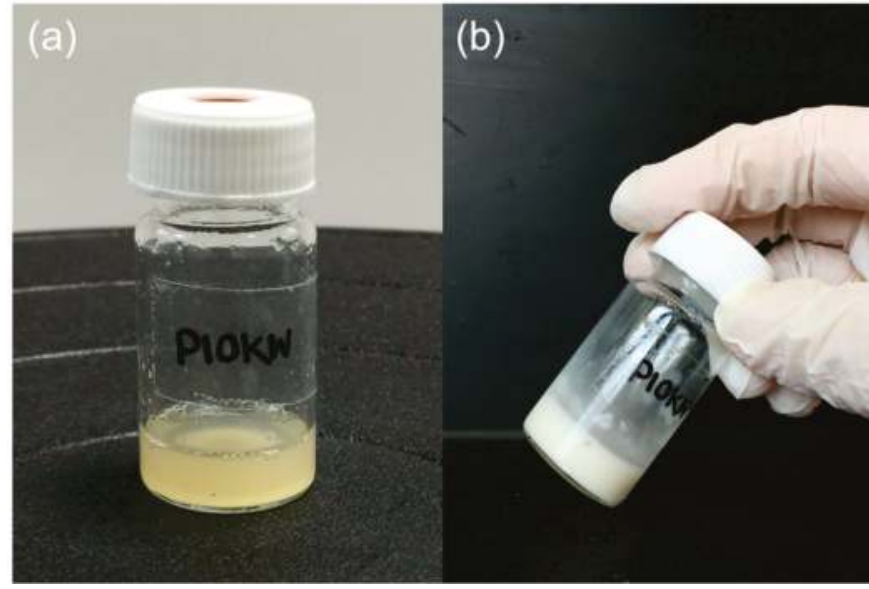

Figure 12. Pectin-PEGMA solution turned white after the addition of $\alpha$-CD: (a) original $10 \%$ P-10K solution; and (b) $10 \%$ P-10K solution after the addition of $\alpha$-CD $(10 \%)$. 


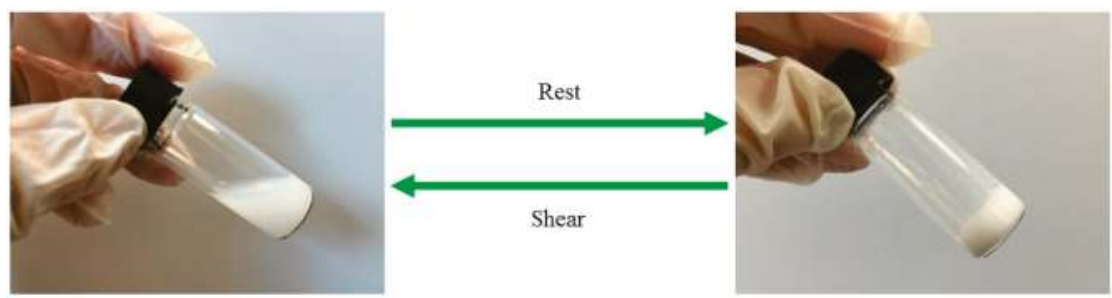

Figure 13. Thixotropic behavior of pectin-PEGMA / $\alpha-C D$ hydrogels.

In conclusion, pectin-PEGMA (P-10K) copolymer was successfully synthesized using cerium-initiated radical polymerization in water. This process is a safer, greener, eco-friendlier way to graft polymers. $\alpha$-CD was incorporated into pectin-PEGMA copolymer and supramolecular gels were formed. The copolymer gelled at low polymer concentration (1\%) with 5\% of $\alpha$-CD. The total concentration of polymer could be lowered to achieve a desired consistency of gel. These supramolecular hydrogels were thixotropic, i.e., the gel could be sheared to liquid and reverted back to gel at rest. Industrially, a thixotropic material is easier to process or transfer without disrupting the consistency. Interestingly, pectin-PEGMA/ $\alpha-C D$ hydrogels were not affected by temperature from 10 to $40{ }^{\circ} \mathrm{C}$, unlike the hydrogels of other PEG-grafted polymers $[15,19,33,34]$. This supramolecular hydrogel has potential to be developed for cosmetic, body and hair care products, or even as an injectable pharmaceutical incipient $[35,36]$. We are currently evaluating the biocompatibility and biodegradability of pectin-PEGMA and exploring the response of this material to other external stimuli.

Supplementary Materials: The following are available online at www.mdpi.com/2073-4360/8/11/404/s1. Figure S1: $500 \mathrm{MHz}{ }^{1} \mathrm{H}$ NMR spectra of the precursors (pectin and 10KPEGMA) and of copolymer P-10K.

Acknowledgments: Siew Yin Chan gratefully acknowledges the funding and support from Monash University Malaysia and the Institute of Materials Research and Engineering, A*STAR. The authors thank Benjamin Qi Yu Chan and Cally Owh for their assistance with illustrations.

Author Contributions: Xian Jun Loh, Wee Sim Choo and David James Young conceived and designed the experiments. Siew Yin Chan and Xian Jun Loh designed the polymers. Siew Yin Chan synthesized and characterized the polymers. Siew Yin Chan, Wee Sim Choo, David James Young and Xian Jun Loh co-wrote the paper. All authors discussed the results and commented on the manuscript.

Conflicts of Interest: The authors declare no conflict of interest.

\section{References}

1. Braconnot, H. Recherches sur un nouvel acide universellement répandu dans tous les végétaux. Annales de Chimie et de Physique 1825, 28, 173-178.

2. Chan, S.-Y.; Choo, W.-S. Effect of extraction conditions on the yield and chemical properties of pectin from cocoa husks. Food Chem. 2013, 141, 3752-3758. [CrossRef] [PubMed]

3. Staunstrup, J. Citrus pectin production and world market. In Proceedings of the International Citrus \& Beverage Conference, Clearwater, FL, USA, 15-18 September 2009.

4. Ciriminna, R.; Chavarría-Hernández, N.; Inés Rodríguez Hernández, A.; Pagliaro, M. Pectin: A new perspective from the biorefinery standpoint. Biofuels Bioprod. Biorefin. 2015, 9, 368-377. [CrossRef]

5. Sharma, R.; Ahuja, M. Thiolated pectin: Synthesis, characterization and evaluation as a mucoadhesive polymer. Carbohydr. Polym. 2011, 85, 658-663. [CrossRef]

6. Sriamornsak, P.; Thirawong, N.; Nunthanid, J.; Puttipipatkhachorn, S.; Thongborisute, J.; Takeuchi, H. Atomic force microscopy imaging of novel self-assembling pectin-liposome nanocomplexes. Carbohydr. Polym. 2008, 71, 324-329. [CrossRef]

7. Günter, E.A.; Popeyko, O.V. Calcium pectinate gel beads obtained from callus cultures pectins as promising systems for colon-targeted drug delivery. Carbohydr. Polym. 2016, 147, 490-499. [CrossRef] [PubMed] 
8. Lupi, F.R.; Gabriele, D.; Seta, L.; Baldino, N.; de Cindio, B.; Marino, R. Rheological investigation of pectin-based emulsion gels for pharmaceutical and cosmetic uses. Rheol. Acta 2014, 54, 41-52. [CrossRef]

9. Da Costa, M.P.M.; de Mello Ferreira, I.L.; de Macedo Cruz, M.T. New polyelectrolyte complex from pectin/chitosan and montmorillonite clay. Carbohydr. Polym. 2016, 146, 123-130. [CrossRef] [PubMed]

10. Codex Alimentarius. General standards for food additives, codex stan 192-1995. In Pectin; Food and Agriculture Organization of the United Nations: Rome, Italy, 2015.

11. Müller-Maatsch, J.; Caligiani, A.; Tedeschi, T.; Elst, K.; Sforza, S. Simple and validated quantitative ${ }^{1} \mathrm{H}$ NMR method for the determination of methylation, acetylation, and feruloylation degree of pectin. J. Agric. Food Chem. 2014, 62, 9081-9087. [CrossRef] [PubMed]

12. Oakenfull, D.; Scott, A. Hydrophobic interaction in the gelation of high methoxyl pectins. J. Food Sci. 1984, 49, 1093-1098. [CrossRef]

13. Gidley, M.J.; Morris, E.R.; Murray, E.J.; Powell, D.A.; Rees, D.A. Evidence for two mechanisms of interchain association in calcium pectate gels. Int. J. Biol. Macromol. 1980, 2, 332-334. [CrossRef]

14. Harada, A.; Li, J.; Kamachi, M. The molecular necklace: A rotaxane containing many threaded $\alpha$-cyclodextrins. Nature 1992, 356, 325-327. [CrossRef]

15. Ren, L.; He, L.; Sun, T.; Dong, X.; Chen, Y.; Huang, J.; Wang, C. Dual-responsive supramolecular hydrogels from water-soluble PEG-grafted copolymers and cyclodextrin. Macromol. Biosci. 2009, 9, 902-910. [CrossRef] [PubMed]

16. Wenz, G.; Han, B.-H.; Müller, A. Cyclodextrin rotaxanes and polyrotaxanes. Chem. Rev. 2006, 106, $782-817$. [CrossRef] [PubMed]

17. Harada, A.; Li, J.; Kamachi, M. Preparation and characterization of a polyrotaxane consisting of monodisperse poly(ethylene glycol) and $\alpha$-cyclodextrins. J. Am. Chem. Soc. 1994, 116, 3192-3196. [CrossRef]

18. Tonelli, A.E. Soluble PEG- $\alpha$-CD-rotaxanes: Where on the PEG chains are the permanently threaded $\alpha$-CDs located and are they mobile? Macromolecules 2008, 41, 4058-4060. [CrossRef]

19. Abdul Karim, A.; Loh, X.J. Design of a micellized $\alpha$-cyclodextrin based supramolecular hydrogel system. Soft Matter 2015, 11, 5425-5434. [CrossRef] [PubMed]

20. Chatjigakis, A.K.; Pappas, C.; Proxenia, N.; Kalantzi, O.; Rodis, P.; Polissiou, M. Ft-ir spectroscopic determination of the degree of esterification of cell wall pectins from stored peaches and correlation to textural changes. Carbohydr. Polym. 1998, 37, 395-408. [CrossRef]

21. Stewart, D.; Morrison, I.M. Ft-ir spectroscopy as a tool for the study of biological and chemical treatments of barley straw. J. Sci. Food Agric. 1992, 60, 431-436. [CrossRef]

22. Wang, H.; Feng, Y.; An, B.; Zhang, W.; Sun, M.; Fang, Z.; Yuan, W.; Khan, M. Fabrication of PU/PEGMA crosslinked hybrid scaffolds by in situ UV photopolymerization favoring human endothelial cells growth for vascular tissue engineering. J. Mater. Sci. 2012, 23, 1499-1510. [CrossRef] [PubMed]

23. Bagheri, M.; Bigdeli, E.; Pourmoazzen, Z. Self-assembled micellar nanoparticles of a novel amphiphilic cholesteryl-poly(L-lactic acid)- $b$-poly(poly(ethylene glycol)methacrylate) block-brush copolymer. Iran. Polym. J. 2013, 22, 293-302. [CrossRef]

24. Ye, H.; Owh, C.; Jiang, S.; Ng, C.; Wirawan, D.; Loh, X. A thixotropic polyglycerol sebacate-based supramolecular hydrogel as an injectable drug delivery matrix. Polymers 2016, 8, 130. [CrossRef]

25. Sun, Y.; Kaplan, J.A.; Shieh, A.; Sun, H.-L.; Croce, C.M.; Grinstaff, M.W.; Parquette, J.R. Self-assembly of a 5-fluorouracil-dipeptide hydrogel. Chem. Commun. 2016, 52, 5254-5257. [CrossRef] [PubMed]

26. Raghavan, S.R.; Hou, J.; Baker, G.L.; Khan, S.A. Colloidal interactions between particles with tethered nonpolar chains dispersed in polar media: Direct correlation between dynamic rheology and interaction parameters. Langmuir 2000, 16, 1066-1077. [CrossRef]

27. Yu, X.; Chen, X.; Chai, Q.; Ayres, N. Synthesis of polymer organogelators using hydrogen bonding as physical cross-links. Colloid Polym. Sci. 2016, 294, 59-68. [CrossRef]

28. Li, J.; Harada, A.; Kamachi, M. Sol-gel transition during inclusion complex formation between $\alpha$-cyclodextrin and high molecular weight poly(ethylene glycol)s in aqueous solution. Polym. J. 1994, 26, 1019-1026. [CrossRef]

29. Li, J.; Li, X.; Ni, X.; Wang, X.; Li, H.; Leong, K.W. Self-assembled supramolecular hydrogels formed by biodegradable PEO-PHB-PEO triblock copolymers and $\alpha$-cyclodextrin for controlled drug delivery. Biomaterials 2006, 27, 4132-4140. [CrossRef] [PubMed] 
30. Li, J. Self-assembled supramolecular hydrogels based on polymer-cyclodextrin inclusion complexes for drug delivery. NPG Asia Mater. 2010, 2, 112-118. [CrossRef]

31. Ye, H.; Owh, C.; Loh, X.J. A thixotropic polyglycerol sebacate-based supramolecular hydrogel showing ucst behavior. RSC Adv. 2015, 5, 48720-48728. [CrossRef]

32. Lucey, J.A.; Tamehana, M.; Singh, H.; Munro, P.A. Stability of model acid milk beverage: Effect of pectin concentration, storage temperature and milk heat treatment. J. Texture Stud. 1999, 30, 305-318. [CrossRef]

33. Huh, K.M.; Ooya, T.; Lee, W.K.; Sasaki, S.; Kwon, I.C.; Jeong, S.Y.; Yui, N. Supramolecular-structured hydrogels showing a reversible phase transition by inclusion complexation between poly(ethylene glycol) grafted dextran and $\alpha$-cyclodextrin. Macromolecules 2001, 34, 8657-8662. [CrossRef]

34. Huh, K.M.; Cho, Y.W.; Chung, H.; Kwon, I.C.; Jeong, S.Y.; Ooya, T.; Lee, W.K.; Sasaki, S.; Yui, N. Supramolecular hydrogel formation based on inclusion complexation between poly(ethylene glycol)-modified chitosan and $\alpha$-cyclodextrin. Macromol. Biosci. 2004, 4, 92-99. [CrossRef] [PubMed]

35. Chee, P.L.; Prasad, A.; Fang, X.; Owh, C.; Yeo, V.J.J.; Loh, X.J. Supramolecular cyclodextrin pseudorotaxane hydrogels: A candidate for sustained release? Mater. Sci. Eng. 2014, 39, 6-12. [CrossRef] [PubMed]

36. Kai, D.; Chua, Y.K.; Jiang, L.; Owh, C.; Chan, S.Y.; Loh, X.J. Dual functional anti-oxidant and SPF enhancing lignin-based copolymers as additives for personal and healthcare products. RSC Adv. 2016, 6, 86420-86427. [CrossRef]

(C) 2016 by the authors. Licensee MDPI, Basel, Switzerland. This article is an open access article distributed under the terms and conditions of the Creative Commons Attribution (CC BY) license (http:/ / creativecommons.org/licenses/by/4.0/). 
Article

\title{
The Effect of Thermal History on the Fast Crystallization of Poly(L-Lactide) with Soluble-Type Nucleators and Shear Flow
}

\author{
Tianfeng Shen, Piming Ma*, Qingqing Yu, Weifu Dong and Mingqing Chen \\ The Key Laboratory of Food Colloids and Biotechnology, Ministry of Education, School of Chemical and \\ Material Engineering, Jiangnan University, 1800 Lihu Road, Wuxi 214122, China; stf0710@126.com (T.S.); \\ yqq1835@163.com (Q.Y.); wfdong@jiangnan.edu.cn (W.D.); mq-chen@jiangnan.edu.cn (M.C.) \\ * Correspondence: p.ma@jiangnan.edu.cn; Tel.: +86-510-85917090
}

Academic Editors: Alexander Böker and Frank Wiesbrock

Received: 14 October 2016; Accepted: 1 December 2016; Published: 10 December 2016

\begin{abstract}
The $N_{1}, N_{1}{ }^{\prime}$-(ethane-1,2-diyl)bis( $N_{2}$-phenyloxalamide) (OXA) is a soluble-type nucleator with a dissolving temperature of $230^{\circ} \mathrm{C}$ in poly(L-lactic acid) (PLLA) matrix. The effect of thermal history and shear flow on the crystallization behavior of the PLLA/OXA samples was investigated by rheometry, polarized optical microscopy (POM), differential scanning calorimetry (DSC), wide angle X-ray diffraction (WAXD), and scanning electron microscopy (SEM). The crystallization process of the PLLA/OXA-240 sample (i.e., pre-melted at $240{ }^{\circ} \mathrm{C}$ ) was significantly promoted by applying a shear flow, e.g., the onset crystallization time $\left(t_{\text {onset }}\right)$ of the PLLA at $155^{\circ} \mathrm{C}$ was reduced from 1600 to $200 \mathrm{~s}$ after shearing at $0.4 \mathrm{rad} / \mathrm{s}$ for even as short as $1.0 \mathrm{~s}$, while the crystallinity $\left(X_{\mathrm{c}}\right)$ was increased to $40 \%$. Moreover, the $t_{\text {onset }}$ of the PLLA/OXA-240 sample is $60 \%-80 \%$ lower than that of the PLLA/OXA-200 sample (i.e., pre-melted at $200^{\circ} \mathrm{C}$ ) with a total shear angle of $2 \mathrm{rad}$, indicating a much higher crystallization rate of the PLLA/OXA-240 sample. A better organization and uniformity of OXA fibrils can be obtained due to a complete pre-dissolution in the PLLA matrix followed by shear and oscillation treatments. The well dispersed OXA fibrils and flow-induced chain orientation are mainly responsible for the fast crystallization of the PLLA/OXA-240 samples. In addition, the shear flow created some disordered $\alpha^{\prime}$-form crystals in the PLLA/OXA samples regardless of the thermal history $\left(200\right.$ or $\left.240{ }^{\circ} \mathrm{C}\right)$.
\end{abstract}

Keywords: poly (L-lactide); crystallization; soluble-type nucleator; shear flow; melting process

\section{Introduction}

Biodegradable and biocompatible poly(L-lactic acid) (PLLA) has recently received more and more attention [1,2]. It is expected to partially solve the environment issues that associated with petrochemical materials [3-5]. The main drawbacks of PLLA-based materials are brittleness, low crystallization rate, low heat resistant temperatures due to the glass transition temperature $\left(T_{g}\right)$ of about $55^{\circ} \mathrm{C}$, and low crystallinity after conventional processing. Notably, the low crystallization rate has restricted the application range of PLLA.

One effective approach to speed up the crystallization of PLLA is by applying nucleating agents to reduce the nucleating activation energy and simultaneously promote the heterogeneous nucleation effect to achieve higher crystallinity. Many nucleating agents have been investigated including talc [6], clay [7], carbon nanotubes [8], and organic additives such as poly(vinylidene fluoride), orotic acid, $N, N$-ethylene-bis(12-hydroxylstearamide) (EBH), nucleobases, substituted-aryl phosphate salts (TMP-5) and $N, N^{\prime}, N^{\prime \prime}$-tricyclohexyl-1,3,5-benzene-tricarboxylamide (TMC-328), $N, N^{\prime}$-bis(benzoyl) hexanedioic acid dihydrazide (TMC-306), and $N_{1}, N_{1}{ }^{\prime}$-(ethane-1,2-diyl) bis $\left(N_{2}\right.$-phenyloxalamide) 
(OXA) [9-22]. Among these nucleating agents, TMC-328, TMC-306, and OXA were proven to have high activity and self-assembly ability [18-22]. However, their solubility and self-assembly behavior are temperature and environment (e.g., static or dynamic conditions) dependent. As a consequence, it is still a challenge to optimize the nucleation effect of the self-organized nucleators.

Another approach to speed up the crystallization of semi-crystalline polymers is by using shear flow which can make polymer chains orientate along the flow direction, resulting in plenty of row nuclei, that thereby enhances crystallization kinetics significantly $[23,24]$. The flow-induced crystallization was mainly applied in polyolefin systems such as poly(ethylene) (PE) and poly(propylene) (PP), and it exists in polymer processing such as injection molding, extrusion, and film blowing [25-27]. Although the environmentally friendly PLLA has been commercialized for more than one decade, the flow-induced crystallization of PLLA has just received limited attention compared with polyolefins $[28,29]$. Furthermore, the effect of nucleating agents in combination with shear flow on the crystallization of PLLA is even less understood [30].

Rheometry was used in the present work to investigate the crystallization behavior of PLLA in the presence of a soluble-type nucleator (OXA) and shear flow. The effect of the thermal history (i.e., melting process) and shear conditions are emphasized and the mechanism of the enhanced crystallization kinetics is discussed. Therefore, the present work not only provides a fundamental research on a complex PLLA/OXA system but also offers a possible approach for high performance PLLA-based products.

\section{Experimental Section}

\subsection{Materials}

Poly(L-lactide) (PLLA, 4032D) was purchased from Nature Works LLC, Minnetonka, MN, USA, with a $M_{\mathrm{n}}=2.1 \times 10^{5} \mathrm{~g} \cdot \mathrm{mol}^{-1}$, PDI $=1.7$, and a D-lactide content of $2 \%$. The nucleating agent, $N_{1}, N_{1}{ }^{\prime}$-(ethane-1,2-diyl)bis( $N_{2}$-phenyloxalamide) (OXA), with a melting temperature of $338{ }^{\circ} \mathrm{C}$ and a purity of $98 \%$, was synthesized in the laboratory with the chemical structure as shown in Figure S1.

\subsection{Sample Preparation}

The PLLA and nucleating agent (OXA) were dried at $60{ }^{\circ} \mathrm{C}$ in a vacuum oven for $12 \mathrm{~h}$ before use. Both of the PLLA/OXA (100/0.5 wt/wt) and neat PLLA samples were prepared in a chamber of a rheometer (HAAKE Polylab-OS, Thermo Fisher Scientific, Bremen, Germany), at $180{ }^{\circ} \mathrm{C}$ and $50 \mathrm{rpm}$ for $5 \mathrm{~min}$. Each sample was then compression molded at $180{ }^{\circ} \mathrm{C}$ and $10 \mathrm{MPa}$ for $2 \mathrm{~min}$ using a hot compression molding machine and subsequently cooled down with room-temperature compression plates at a pressure of $5 \mathrm{MPa}$ to make disk-shaped samples with a diameter of $25 \mathrm{~mm}$ and a thickness of $1.0 \mathrm{~mm}$. The disk-shaped samples were further dried in vacuum at $60^{\circ} \mathrm{C}$ for $12 \mathrm{~h}$ before the rheological measurements.

\subsection{Characterization}

Differential Scanning Calorimetry (DSC): The crystallization and melting behaviour of the samples were studied by using DSC (DSC 8000, Perkin Elmer, Waltham, MA, USA). Each sample was heated to $200{ }^{\circ} \mathrm{C}$ at $10{ }^{\circ} \mathrm{C} / \mathrm{min}$, held for $3 \mathrm{~min}$ at $200^{\circ} \mathrm{C}$, then cooled to $0^{\circ} \mathrm{C}$ and re-heated to $200^{\circ} \mathrm{C}$ at $10{ }^{\circ} \mathrm{C} / \mathrm{min}$. The crystallinity of PLLA $\left(X_{\mathrm{c}}\right)$ is calculated via $X_{c}=\left(\Delta H_{m}-\Delta H_{c c}\right) /\left(\omega * \Delta H_{m}^{0}\right) \times$ $100 \%$ [31], where $\Delta H_{\mathrm{m}}$ and $\Delta H_{\mathrm{cc}}$ are the measured melt and cold crystallization enthalpy of the PLLA, respectively, $\omega$ is the weight fraction of the PLLA in the blends, and $\Delta H_{m}^{0}=93.6 \mathrm{~J} / \mathrm{g}$ is the melting enthalpy of $100 \%$ crystalline PLLA [32]. All tests were carried out in a nitrogen atmosphere.

Polarized optical microscopy (POM): The crystal morphology of the PLA/OXA samples upon cooling from the melt $\left(200\right.$ and $240{ }^{\circ} \mathrm{C}$, respectively) were monitored with a POM (Axio Scope 1, Carl Zeiss, Oberkochen, Germany) in combination with a Linkam THMS600 hot-stage. Each sample 
was sandwiched between two carefully cleaned glass slides and was first held at 200 or $240{ }^{\circ} \mathrm{C}$ for $3 \mathrm{~min}$ and then cooled to room temperatures at $10^{\circ} \mathrm{C} / \mathrm{min}$. Images were taken at varied temperatures.

Rheology: Rheological experiments were carried out on a DHR-2 rheometer (TA Instruments, New Castle, DE, USA) in a plate-plate configuration ( $25 \mathrm{~mm}$ in diameter and $1 \mathrm{~mm}$ in gap) to study the isothermal crystallization of the PLLA and PLLA/OXA samples with and without pre-shear treatment. The experimental procedures for the shear-induced crystallization are illustrated in Figure 1 and were performed as follows: (1) the samples were annealed at 200 or $240{ }^{\circ} \mathrm{C}$ ( $\left.T_{1}\right)$ for $3 \mathrm{~min}$; (2) subjected to a dynamic temperature sweep with a ramp of $-5{ }^{\circ} \mathrm{C} / \mathrm{min}$ to the desired crystallization temperature $\left(T_{2}=155^{\circ} \mathrm{C}\right)$; (3) a shear pulse with controlled shear rates and shear time was applied on each sample; (4) an oscillatory time sweep was performed at $T_{2}$ to trace the evolution of the storage modulus of the samples upon the isothermal crystallization. The strain and frequency were set at $1 \%$ and $1 \mathrm{~Hz}$, respectively for the oscillatory time sweep.

The PLLA/OXA samples treated at 200 and $240{ }^{\circ} \mathrm{C}\left(T_{1}\right)$ are abbreviated as PLLA/OXA-200 and PLLA/OXA-240, respectively.

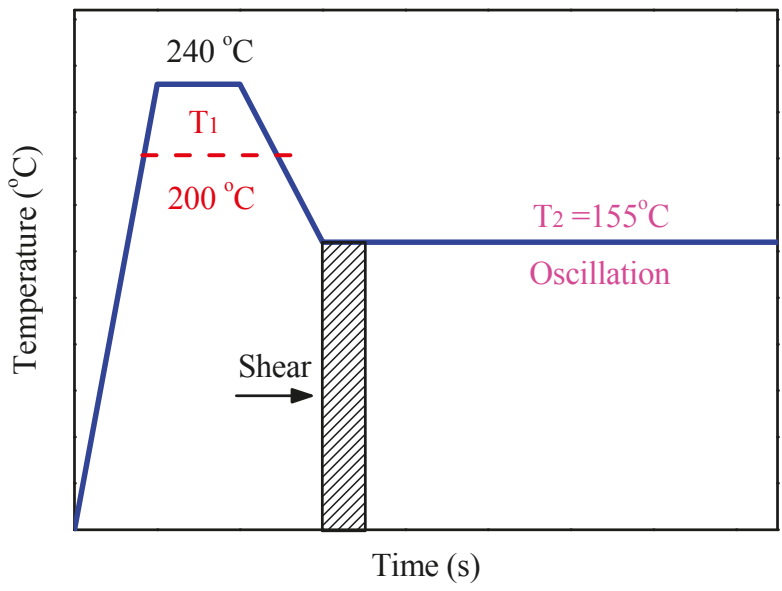

Figure 1. Procedure indications of thermal and shear applications for the rheological measurement of the PLLA/OXA samples.

Wide Angle X-ray Diffraction (WAXD): WAXD measurements were carried out by using an X-ray diffractometer (Bruker AXS D8, Karlsruhe, Germany) equipped with a Ni-filtered Cu K $\alpha$ radiation source and with a wavelength of $1.542 \AA$. The measurements were operated at $40 \mathrm{kV}$ and $40 \mathrm{~mA}$ with scan angles from $5^{\circ}$ to $50^{\circ}$ and a scan rate of $3^{\circ} / \mathrm{min}$.

Scanning Electron Microscopy (SEM): The micro-morphology of the sheared PLLA/OXA samples was observed by using a SEM (S-4800, Hitachi, Tokyo, Japan) at an accelerating voltage of $2 \mathrm{kV}$. The cross sections of each sample obtained by cryo-fracture were etched with a 1:2 water-methanol mixture containing $0.025 \mathrm{~mol} / \mathrm{L} \mathrm{NaOH}$ and were subsequently coated with a thin gold layer before observation.

\section{Results and Discussion}

\subsection{Effect of OXA on the Crystallization of the PLLA under Static Conditions}

The effect of OXA on the non-isothermal crystallization and melting behavior of PLLA was studied with DSC, as shown in Figure 2. The corresponding thermal parameters are provided in the Supporting Information (Table S1). No crystallization traces of the PLLA were detected upon cooling (Figure 2a) followed by a pronounced cold crystallization peak in the subsequent heating 
scan $\left(T_{\mathrm{cC}}=112.4{ }^{\circ} \mathrm{C}, \Delta H_{\mathrm{cc}}=-28.4 \mathrm{~J} / \mathrm{g}\right.$, Figure $\left.2 \mathrm{~b}\right)$. Huneault et al. reported a $T_{\mathrm{c}}$ of $103.2^{\circ} \mathrm{C}$ for PLLA with talc as a nucleating agent (cooling at $10{ }^{\circ} \mathrm{C} / \mathrm{min}$ ) [6], while Nam et al. reported a $T_{\mathrm{C}}$ of $110^{\circ} \mathrm{C}$ for EBH-nucleated PLLA (cooling at $2{ }^{\circ} \mathrm{C} / \mathrm{min}$ ) [10]. These results demonstrate a poor crystalline capability of PLLA due to chain stiffness and the lack of efficient nucleators [33,34]. A high $T_{\mathrm{c}}$ and a sharp crystallization peak correspond to a high crystallization rate. Therefore, the DSC data in Figure 2 indicate that the OXA could speed up the crystallization of the PLLA with a narrow crystallization peak $\left(T_{\mathrm{C}}=116.2{ }^{\circ} \mathrm{C}, X_{\mathrm{C}}=34.3 \%\right)$. A multi-melting peak behavior can be resulted from different crystalline forms or the same crystalline forms with different perfections. It is reported that PLLA can crystallize in three different forms depending on the crystallization conditions ( $\alpha, \beta, \gamma$ forms) [35-39]. The $\alpha$ form is the most common in PLLA, while $\beta$ and $\gamma$ forms can occur due to special processing conditions. Actually, the $\beta$ and $\gamma$ forms should not exist in the present samples as indicated by the XRD results (see below with shear-0). Therefore, a double melting peak of PLLA may be associated with the different crystallization conditions between the $\alpha$ and $\alpha^{\prime}$ (also noted as $\delta$ ) crystals. When PLLA was crystallized at temperatures that were more suitable for $\alpha^{\prime}$ crystal formation (e.g., around $T_{\text {cc }}$ in this work), metastable $\alpha^{\prime}$ crystals were formed, and parts of them transformed into stable $\alpha$ crystallites with a higher melting temperature upon heating, leading to the double melting behaviors in the second run of DSC [35].Thus, the double endothermic peaks of PLLA in this work are assigned to a melting/re-crystallization/re-melting mechanism. The $T_{\mathrm{m} 1}$ of PLLA/OXA is slightly higher than that of PLLA (Figure $2 b$ ) indicating a better organization and uniformity of PLLA crystals in the presence of OXA.
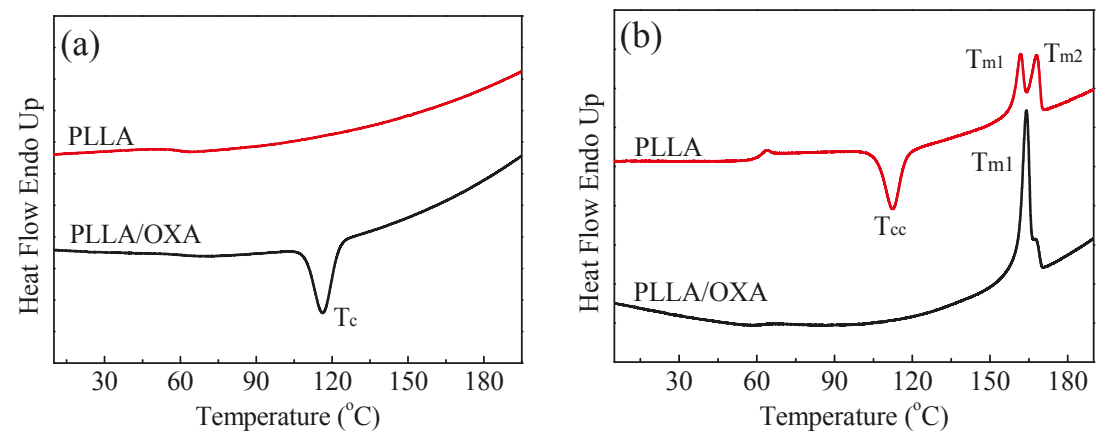

Figure 2. Differential scanning calorimetry (DSC) curves of the PLLA and PLLA/OXA samples: (a) cooling from the melt and (b) subsequent heating processes. The cooling and heating rates are $10^{\circ} \mathrm{C} / \mathrm{min}$.

The effect of OXA on the isothermal crystallization of PLLA was studied in the temperature range of $130-145^{\circ} \mathrm{C}$. Figure 3 shows the relative crystallinity $\left(X_{\mathrm{t}}\right)$ as a function of crystallization time $(t)$ and the half-life crystallization time $\left(t_{1 / 2}\right)$ as a function of temperature $\left(T_{\mathrm{c}}\right)$. The crystallization time of the PLLA/OXA sample is shorter in comparison with that of the PLLA. Taking $T_{\mathrm{C}}=135{ }^{\circ} \mathrm{C}$ as an example, the $t_{1 / 2}$ of neat PLLA was $31 \mathrm{~min}$ in comparison with $4.5 \mathrm{~min}$ of the PLLA/OXA sample. These results indicate that the OXA significantly accelerated the isothermal crystallization process of PLLA. On the other hand, the $t_{1 / 2}$ of PLLA/OXA increased with increasing $T_{\mathrm{C}}$ because of the difficulty in nucleation at high(er) temperatures. It has to be noted that no crystallization of PLLA/OXA occurred within $90 \mathrm{~min}$ at $145^{\circ} \mathrm{C}$ (see Figure S2). In literature, crystallization of PLLA was not observed at $146^{\circ} \mathrm{C}$ even in the presence of poly (D-lactic acid) as a nucleating agent [40]. Thus, it would be more difficult for the crystallization of PLLA/OXA at a temperature higher than $145^{\circ} \mathrm{C}$. 

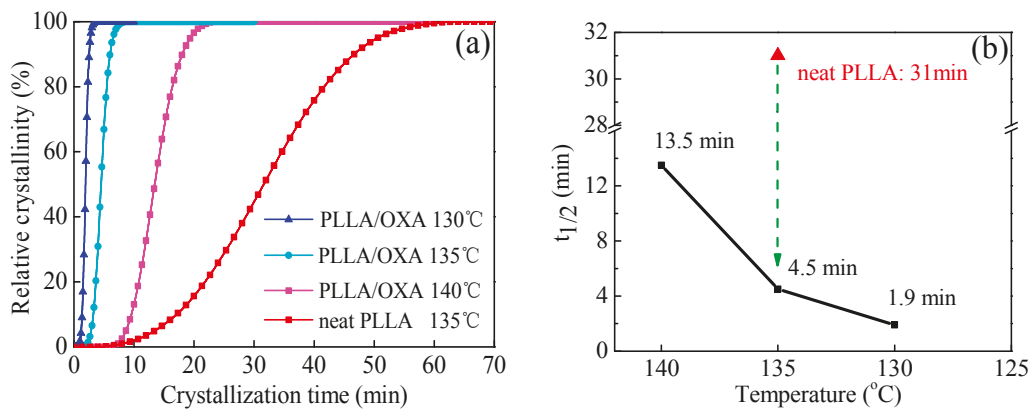

Figure 3. (a) The relative crystallinity $\left(X_{\mathrm{t}}\right)$ of PLLA and PLLA/OXA as a function of crystallization time $(t)$, and (b) the corresponding half-life crystallization time $\left(t_{1 / 2}\right)$ obtained at $X_{t}=50 \%$.

\subsection{Self-Organization of the OXA upon Cooling from Different Temperatures}

Since rheological responses are sensitive to microstructural changes [41,42], they are used to investigate the self-organization behavior of OXA in the PLLA melt and the crystallization behavior of PLLA. It is known that the dissolution temperature of OXA in the PLLA melt is around $230{ }^{\circ} \mathrm{C}$ [22]. Figure 4 shows the variation of the storage modulus $\left(G^{\prime}\right)$ of the PLLA/OXA samples upon cooling from 200 and $240{ }^{\circ} \mathrm{C}$, respectively. For the PLLA/OXA-240 sample, two steep increases in storage modulus are observed around 195 and $145{ }^{\circ} \mathrm{C}$, respectively. Two polarized optical microscopy (POM) images of the PLLA/OXA-240 samples taken upon cooling are presented as insets in Figure 4a,b, which clearly confirmed the self-organized OXA fibrillar superstructures. Therefore, the strong increase at $195{ }^{\circ} \mathrm{C}$ is associated with the self-organization process of the dissolved OXA into a non-soluble fibrillar network, while the increase at $145^{\circ} \mathrm{C}$ corresponds to the crystallization of the PLLA matrix. Similar phenomena were observed in PLA/TMC-306 systems as well [18]. In the case of the PLLA/OXA-200 sample, an inconspicuous increase of $G^{\prime}$ occurred at around $190^{\circ} \mathrm{C}$ followed also by a strong increase around $125^{\circ} \mathrm{C}$. As OXA could only be partially dissolved in the PLLA matrix at $200{ }^{\circ} \mathrm{C}$ (Image c), the former increase of $G^{\prime}$ associated with the self-organization of some dissolved OXA is not obvious. The increase at $125^{\circ} \mathrm{C}$ also resulted from the crystallization of the PLLA matrix. It is noticed that the crystallization temperature of the PLLA/OXA-240 sample is $20^{\circ} \mathrm{C}$ higher than that of the PLLA/OXA-200 sample. Apparently, the melting process is an important factor in the crystallization of PLLA/OXA, which is studied further in the presence of shear flow (see below).

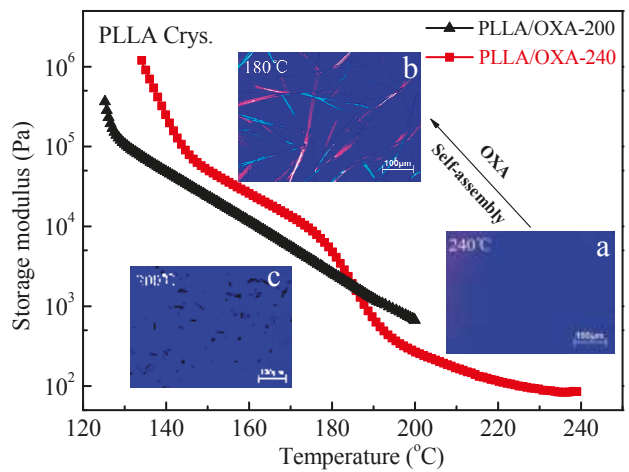

Figure 4. Storage modulus versus temperature for the PLLA/OXA samples upon cooling from 200 and $240{ }^{\circ} \mathrm{C}$, respectively. Three POM images performed under static conditions are shown: (a) PLLA/OXA-240 at $240{ }^{\circ} \mathrm{C}$; (b) PLLA/OXA-240 at $180{ }^{\circ} \mathrm{C}$; and (c) PLLA/OXA-200 at $200{ }^{\circ} \mathrm{C}$. 


\subsection{Effect of the Melting Process and Shear Flow on the Crystallization Behaviors of the PLLA/OXA Samples}

Effect of shear rate. Figure 5a shows the $G^{\prime}$ evolutions of the PLLA/OXA-240 samples during the shear-induced isothermal crystallization at $155^{\circ} \mathrm{C}$. A series of shear rates $(\gamma=0.1,0.2,0.3,0.4$, and $0.5 \mathrm{rad} / \mathrm{s}$ ) were examined while the overall shear angle was fixed at 2 rad by adjusting the shear time. The data for the non-sheared sample $(\gamma=0.0 \mathrm{rad} / \mathrm{s})$ is plotted for comparison in Figure 5a. For the non-sheared sample, the $G^{\prime}$ slowly rose with time. Impressively, the curve of $G^{\prime} \sim$ time of the PLLA/OXA-240 samples shifted to a shorter time side rapidly when a shear was applied, regardless of the shear rate. These results indicate a faster overall crystallization rate of the PLLA/OXA sample after melting at $240{ }^{\circ} \mathrm{C}$ and shearing at $155^{\circ} \mathrm{C}$.

The inflection point of $G^{\prime} \sim$ time is defined as the onset crystallization time $\left(t_{\text {onset }}\right)$, which is plotted as a function of shear rate for the PLLA/OXA samples that cooled from both temperatures, as shown in Figure $5 \mathrm{~b}$. The $t_{\text {onset }}$ of the PLLA/OXA-240 samples is reduced from $1600 \mathrm{~s}$ to around $300 \mathrm{~s}$ by increasing the shear rate from 0 to $0.1 \mathrm{rad} / \mathrm{s}$ and then leveled off, indicating a remarkable acceleration of the crystallization kinetics. The acceleration is mainly contributed by the promoted nucleation process because the crystallization was performed at the same temperature $\left(155^{\circ} \mathrm{C}\right)$ and the crystal growth rate of a polymeric material is usually the same at a certain temperature. Figure $5 \mathrm{~b}$ also shows that the $t_{\text {onset }}$ of the PLLA/OXA-240 samples is much shorter than that of the PLLA/OXA-200 samples at the same shear rate, and the differences between the $t_{\text {onset }}$ values of the PLLA/OXA-240 and the PLLA/OXA-200 samples are larger at lower shear rates. The non-sheared PLLA/OXA-200 sample did not show inflection point ( $t_{\text {onset }}$ ) within a couple of hours (data are not shown here). Therefore, it can be concluded from these results that a high(er) melting temperature (e.g., $240{ }^{\circ} \mathrm{C}$ ) and a shear flow are both beneficial to the fast crystallization of PLLA/OXA samples.
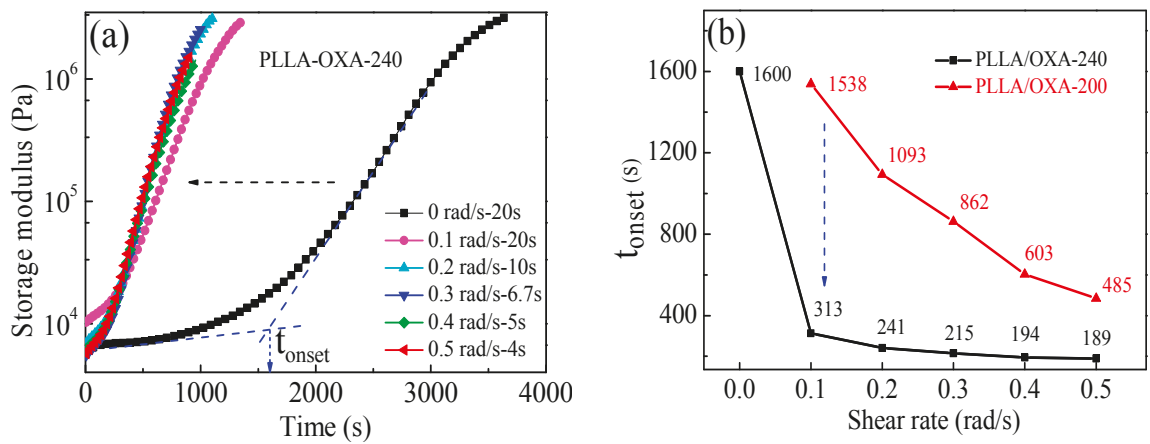

Figure 5. (a) Storage modulus of the PLLA/OXA-240 samples as a function of the crystallization time at $155^{\circ} \mathrm{C}$ and the shear rate; (b) the $t_{\text {onset }}$ values of the PLLA/OXA samples as a function of the shear rate.

The crystallized PLLA/OXA-240 samples were collected after the rheological experiments for differential scanning calorimetry (DSC) characterization, shown in Figure S3 and Table S2. It was found that the crystallinity of the PLLA increased monotonically with increasing shear rate, while the melting temperature remained constant.

Effect of shear time. A shear rate of $0.4 \mathrm{rad} / \mathrm{s}$ was selected to further study the effect of shear time on the crystallization of the PLLA/OXA samples. Figure 6a shows the variations of $G^{\prime}$ of the PLLA/OXA-240 samples as a function of the shearing time and the crystallization time at $155{ }^{\circ} \mathrm{C}$. The corresponding $t_{\text {onset }}$ as a function of shear time is plotted in Figure $6 \mathrm{~b}$. The sheared sample $(0.4 \mathrm{rad} / \mathrm{s}$ for $0.5 \mathrm{~s})$ shows a much sharper rise of $G^{\prime}$ in comparison with the non-sheared sample, demonstrating a rapid overall crystallization process after the shear. However, the $G^{\prime} \sim$ time curves did not shift any more when the shear time was longer than $5 \mathrm{~s}$. It implies that the effective orientation of microstructures might become dynamically balanced after a critical shear time $\left(t_{\mathrm{c}}\right)$. The $t_{\mathrm{c}}$ value was 
$1-5 \mathrm{~s}$ for the PLLA/OXA-240 sample at a shear rate of $0.4 \mathrm{rad} / \mathrm{s}$. The $G^{\prime} \sim$ time of the PLLA/OXA-200 samples showed a similar trend as a function of shear time (data are not shown here), whereas the $t_{\mathrm{c}}$ value was $15-20 \mathrm{~s}$ at the same shear rate.

Similar to the effect of the shear rate, the $t_{\text {onset }}$ of the PLLA/OXA-240 samples reduced by $\sim 90 \%$ when a shear time of $1 \mathrm{~s}$ was applied, and then leveled off (Figure 6b). In comparison, the PLLA/OXA-200 sample had a much longer $t_{\text {onset }}$ value, notably with a short(er) shear time. Taking a shear time of $5 \mathrm{~s}$ as an example, the $t_{\text {onset }}$ of the PLLA/OXA-200 sample was 2 times larger than that of the PLLA/OXA-240 sample. These results further confirm that a higher melting temperature in combination with a shear flow can more effectively promote the crystallization process of PLLA.
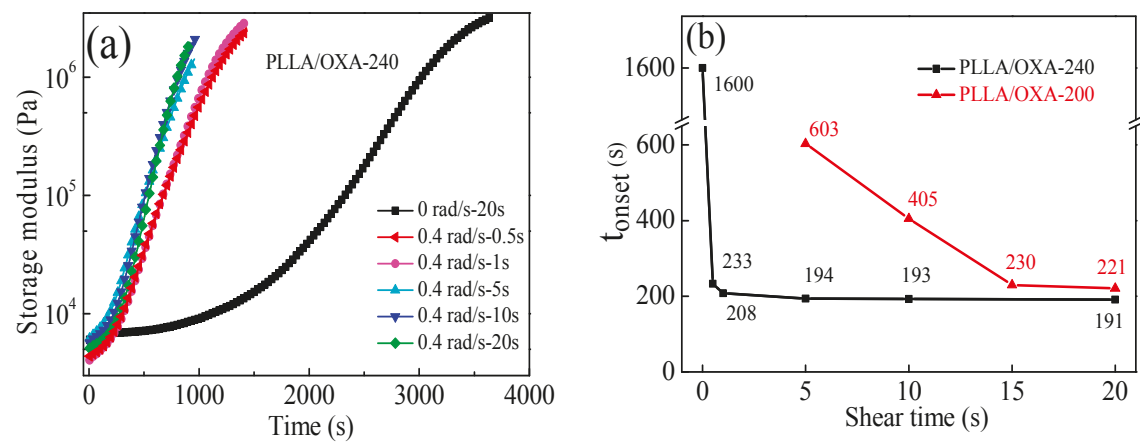

Figure 6. (a) Storage modulus of the PLLA/OXA-240 samples as a function of shear time and isothermal crystallization time at $155{ }^{\circ} \mathrm{C}$ and $(\mathbf{b})$ the $t_{\text {onset }}$ values of the PLLA/OXA samples as a function of shear time. The shear rate is fixed at $0.4 \mathrm{rad} / \mathrm{s}$ for all samples and the PLLA/OXA-200 sample without shear did not crystallize within the experimental time span (90 min).

\subsection{Crystal Structure and Morphology of the PLLA/OXA Samples}

In order to gain deeper insight into the effect of the melt process and shear flow on the crystallization of PLLA, wide angle X-ray diffraction (WAXD) measurements were carried out and the diffraction patterns are shown in Figure 7. For the non-sheared PLLA/OXA samples, three diffraction peaks at $2 \theta=16.7^{\circ}, 19.2^{\circ}$, and $22.6^{\circ}$ were detected, correlating to the $200 / 110,203$, and 105 planes of PLLA $\alpha$ form crystals, respectively [35,43,44]. Meanwhile, a broad diffraction peak was observed for both of the non-sheared PLLA/OXA-200 and PLLA/OXA-240 samples, indicating an incomplete crystallization of the PLLA phase. The peak intensity of the PLLA/OXA-240 sample at $2 \theta=16.7^{\circ}$ was larger than that of the PLLA/OXA-200 sample, indicating a relatively higher crystallinity of the PLLA/OXA-240 sample. Intriguingly, the diffraction peaks were elevated when a shear flow was applied, accompanied by the disappearance of the broad diffraction peak. Meanwhile, the diffraction peaks of the PLLA/OXA samples shifted by $0.3^{\circ}$ to smaller $2 \theta$ positions after applying the shear flow regardless of the melting temperatures $\left(T_{1}=200\right.$ or $\left.240^{\circ} \mathrm{C}\right)$. A similar result was observed in nucleator-modified PLLA fibers where the shift was ascribed to the existence of some disordered $\alpha^{\prime}$-form crystals $[45,46]$. It has been proven that $\alpha$ - and $\alpha^{\prime}$-form crystals share the same $10_{3}$ helix chain conformation and orthorhombic unit cell, but the packing of the side groups in the helical chains of the $\alpha^{\prime}$-form crystals is less ordered and looser than that of the $\alpha$-form crystals [36]. 


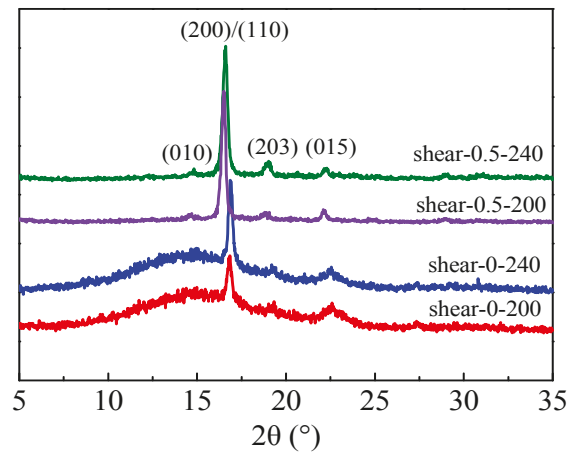

Figure 7. X-ray diffraction patterns of the PLLA/OXA-200 and PLLA/OXA-240 samples with and without a shear flow $(0.4 \mathrm{rad} / \mathrm{s}$ for $5 \mathrm{~s})$ at $155{ }^{\circ} \mathrm{C}$. The samples were taken after the rheology measurements.

The microstructures of the sheared PLLA/OXA samples taken from different crystallization periods were studied using SEM, as shown in Figure 8. The OXA molecules in the PLLA melt can self-organize into fibrils via hydrogen bonding, and are capable of serving as nucleating agents [21]. The self-organized fibrils reassembled into larger needle-like superstructures after a shear flow, as shown in Figure 8a,c. It was observed that the OXA in the PLLA/OXA-240 sample showed better self-organization and alignment in comparison with that in the PLLA/OXA-200 sample. These superstructures were subsequently re-dispersed into even smaller fibrils due to the oscillation effect during the subsequent isothermal crystallization process (Figure $8 \mathrm{~b}, \mathrm{~d}$ ). The better dispersed OXA fibrils provided extra nucleating sites for the accumulation of PLLA crystals, leading to a type of shish-kebab crystal morphology (area A in Figure 8d). Moreover, the PLLA/OXA-240 sample showed more and thicker PLLA crystals in comparison with the PLLA/OXA-200 sample (Figure 8b,d).

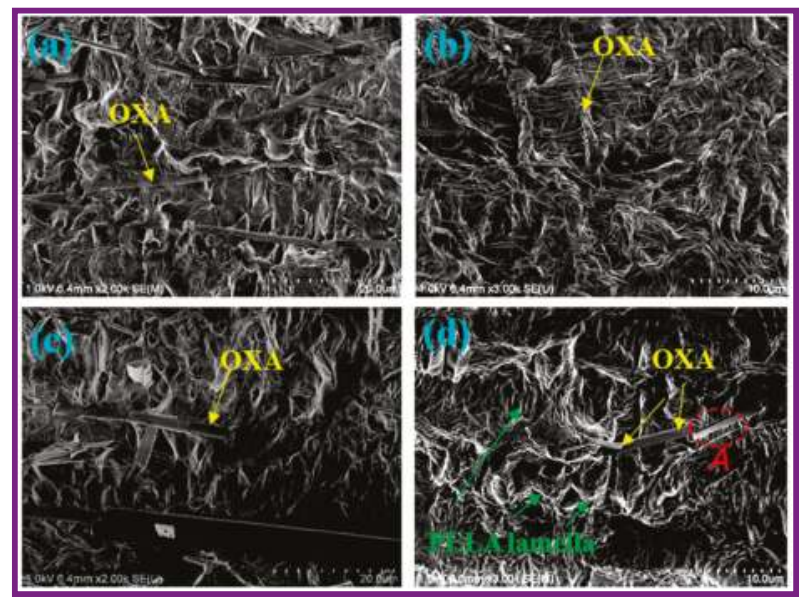

Figure 8. SEM images of the PLLA/OXA samples: (a) PLLA/OXA-200 just after shear; (b) PLLA/OXA-200 after shear and crystallization; (c) PLLA/OXA-240 just after shear and (d) PLLA/OXA-240 after shear and crystallization. The same shear condition, i.e., $0.4 \mathrm{rad} / \mathrm{s}$ for $5 \mathrm{~s}$ was applied to each sample and the crystallization is performed under oscillation conditions. A larger magnification of Images $(\mathbf{b}, \mathbf{d})(3000 \times)$ was used compared with that of Images $(\mathbf{a}, \mathbf{c})(2000 \times)$, for better visualization purposes. 


\subsection{Mechanism Discussion}

The above results clearly show that the PLLA/OXA-240 samples have better crystallization ability than the PLLA/OXA-200 sample under the same shear and crystallization conditions, and are also better than the non-sheared PLLA/OXA-240 sample. Therefore, a schematic illustration is provided for the mechanism discussion of shear flow induced crystallization of the PLLA/OXA-240 system (Figure 9). At $240^{\circ} \mathrm{C}$, the OXA can be melted and well dissolved in the PLLA matrix, as confirmed by Figure $4 \mathrm{a}$ and illustrated in Figure 9a. Upon cooling from 240 to $155^{\circ} \mathrm{C}$, the dissolved OXA molecules can self-organize more homogeneously than the original OXA aggregates (e.g., cooling from $200{ }^{\circ} \mathrm{C}$ ) into fibrils/needle-like superstructures that are capable of serving as nucleating sites which, however, were randomly distributed. At high temperatures (e.g., $155^{\circ} \mathrm{C}$ ), PLLA without shear is difficult to crystallize even in the presence of the OXA fibrils [20-22]. However, the fibrils and PLLA molecules gradually orientate in the shear direction when a shear flow is applied, see Figure 9c,d. The fibrils superstructures become finer in dimension and are better dispersed in the PLLA matrix due to the subsequent oscillation of the rheometer, providing more nucleating sites (Figure 9e). It is believed that the oriented polymer chains could assemble into a parallel array and form the precursors of primary nuclei for crystallization $[23,24]$. Therefore, the crystallization kinetics of the PLLA is promoted both by the evolution of the OXA superstructures and by a certain extent of the PLLA chain orientation.

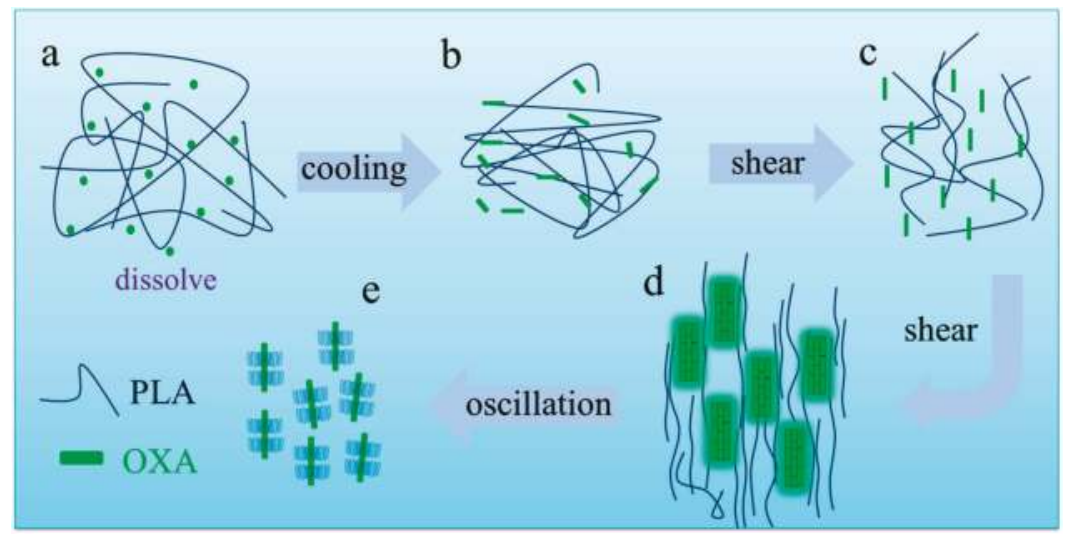

Figure 9. Schematic illustration of the enhanced crystallization kinetics of the PLLA/OXA-240 systems showing the evolution of the OXA superstructures and the orientation of the PLLA macromolecules in the presence of shear flow. (a) PLLA/OXA melt with dissolved OXA molecules; (b) self-organization of OXA in the PLLA melt; (c,d) the OXA fibrils and PLLA molecules gradually orientate in the shear direction; (e) crystallization of PLLA.

\section{Conclusions}

$N_{1}, N_{1}{ }^{\prime}$-(ethane-1,2-diyl)bis( $N_{2}$-phenyloxalamide) (OXA) was identified as a soluble-type nucleator for poly(L-lactic acid) (PLLA). In the present work, both OXA and the shear flow were applied to accelerate the crystallization of PLLA, at two different melt annealing temperatures (200 and $240{ }^{\circ} \mathrm{C}$ ). The effect of melting temperature and shear flow on the crystallization of the PLLA/OXA samples at $155^{\circ} \mathrm{C}$ was investigated by using rheometry, polarized optical microscopy (POM), differential scanning calorimetry (DSC), wide angle X-ray diffraction (WAXD) and scanning electron microscopy (SEM). As a result, the crystallization of the PLLA/OXA-240 sample was significantly sped up by even a gentle shear flow, e.g., the onset crystallization time ( $t_{\text {onset }}$ ) of the PLLA could be reduced by $\sim 90 \%$ with a shear flow as small as $0.4 \mathrm{rad}$, while the crystallinity $\left(X_{\mathrm{c}}\right)$ reached $40 \%$. 
Compared with the PLLA/OXA-200 sample, the $t_{\text {onset }}$ of the PLLA/OXA-240 sample was reduced by $60 \%-80 \%$ under the same shear conditions (a total shear angle of $2 \mathrm{rad}$ ). Therefore, the higher melting temperature $\left(240^{\circ} \mathrm{C}\right)$ does accelerate the crystallization of PLLA in the presence of OXA and shear flow. OXA can be dissolved completely in the PLLA matrix at $240{ }^{\circ} \mathrm{C}$, while only partially dissolved at $200{ }^{\circ} \mathrm{C}$. A better organization and uniformity of the OXA superstructures can be achieved due to the complete pre-dissolution in the PLLA matrix and a subsequent shear and oscillation treatment. The well dispersed OXA fibrils and shear flow induced PLLA chain orientation are responsible for the fast crystallization of the PLLA/OXA-240 samples. In addition, the X-ray diffraction patterns showed that the shear flow created some disordered $\alpha^{\prime}$-form crystals in the PLLA/OXA samples regardless of the melting temperatures $\left(200\right.$ or $\left.240^{\circ} \mathrm{C}\right)$. The new findings in this work may be applicable to other OXA-nucleated polymeric systems as well, and thus may expand the application range of OXA and PLLA.

Supplementary Materials: The following are available online at www.mdpi.com/2073-4360/8/12/431/s1. Figure S1: The chemical structure of the OXA, Figure S2: DSC heat flow as a function of isothermal crystallization time and temperatures for the PLLA/OXA (100/0.5 wt/wt) samples, Figure S3: First heating DSC curves of the sheared PLLA/OXA-240 samples after the rheological experiments at $155{ }^{\circ} \mathrm{C}$, Table S1: Thermal parameters of the PLLA and PLLA/OXA samples obtained from the DSC cooling and 2nd heating scans, Table S2: Thermal parameters of the PLLA/OXA-240 samples derived from Figure S3.

Acknowledgments: This work was supported by the Natural Science Foundation of Jiangsu Province (BK20130147), the National Natural Science Foundation of China (51573074, 51303067), and the Fundamental Research Funds for the Central Universities (JUSRP51624A).

Author Contributions: Tianfeng Shen and Qingqing Yu performed experiments, wrote the manuscript and prepared the figures and tables. Piming Ma initiated and guided the work and revised the manuscript. Weifu Dong and Mingqing Chen provided discussion and suggestions on this work.

Conflicts of Interest: The authors declare no conflict of interest.

\section{References}

1. Rasal, R.M.; Janorkar, A.V.; Hirt, D.E. Poly(lactic acid) modifications. Prog. Polym. Sci. 2010, 35, 338-356. [CrossRef]

2. Reddy, M.M.; Vivekanandhan, S.; Misra, M.; Bhatia, S.K.; Mohanty, A.K. Biobased plastics and bionanocomposites: Current status and future opportunities. Prog. Polym. Sci. 2013, 38, 1653-1689. [CrossRef]

3. Nampoothiri, K.M.; Nair, N.R.; John, R.P. An overview of the recent developments in polylactide (PLA) research. Bioresour. Technol. 2010, 101, 8493-8501. [CrossRef] [PubMed]

4. Liu, H.; Zhang, J. Research progress in toughening modification of poly(lactic acid). J. Polym. Sci. Polym. Phys. 2011, 49, 1051-1083. [CrossRef]

5. Raquez, J.M.; Habibi, Y.; Murariu, M.; Dubois, P. Polylactide (PLA)-based nanocomposites. Prog. Polym. Sci. 2013, 38, 1504-1542. [CrossRef]

6. Li, H.; Huneault, M.A. Effect of nucleation and plasticization on the crystallization of poly(lactic acid). Polymer 2007, 48, 6855-6866. [CrossRef]

7. Krikorian, V.; Pochan, D.J. Unusual crystallization behavior of organoclay reinforced poly(L-lactic acid) nanocomposites. Macromolecules 2004, 37, 6480-6491. [CrossRef]

8. Xu, Z.; Zhang, Y.; Wang, Z.; Sun, N.; Li, H. Enhancement of electrical conductivity by changing phase morphology for composites consisting of polylactide and poly( $\varepsilon$-caprolactone) filled with acid-oxidized multiwalled carbon nanotubes. ACS Appl. Mater. Interface 2011, 3, 4858-4864. [CrossRef] [PubMed]

9. Sun, Y.; He, C. Synthesis and stereocomplex crystallization of poly(lactide)-graphene oxide nanocomposites. ACS Macro. Lett. 2012, 1, 709-713. [CrossRef]

10. Nam, J.Y.; Okamoto, M.; Okamoto, H.; Nakano, M.; Usuki, A.; Matsuda, M. Morphology and crystallization kinetics in a mixture of low-molecular weight aliphatic amide and polylactide. Polymer 2006, 47, 1340-1347. [CrossRef]

11. Pan, P.; Shan, G.; Bao, Y. Enhanced nucleation and crystallization of poly (L-lactic acid) by immiscible blending with poly(vinylidene fluoride). Ind. Eng. Chem. Res. 2014, 53, 3148-3156. [CrossRef] 
12. Brochu, S.; Prud'Homme, R.E.; Barakat, I.; Jerome, R. Stereocomplexation and morphology of polylactides. Macromolecules 1995, 28, 5230-5239. [CrossRef]

13. Schmidt, S.C.; Hillmyer, M.A. Polylactide stereocomplex crystallites as nucleating agents for isotactic polylactide. J. Polym. Sci. Polym. Phys. 2001, 39, 300-313. [CrossRef]

14. Yamane, H.; Sasai, K. Effect of the addition of poly(D-lactic acid) on the thermal property of poly(L-lactic acid). Polymer 2003, 44, 2569-2575. [CrossRef]

15. Qiu, Z.; Li, Z. Effect of orotic acid on the crystallization kinetics and morphology of biodegradable poly(L-lactide) as an efficient nucleating agent. Ind. Eng. Chem. Res. 2011, 50, 12299-12303. [CrossRef]

16. Shi, Y.; Shao, L.; Yang, J.; Huang, T.; Wang, Y.; Zhang, N.; Wang, Y. Highly improved crystallization behavior of poly(L-lactide) induced by a novel nucleating agent: Substituted-aryl phosphate salts. Polym. Adv. Technol. 2013, 24, 42-50. [CrossRef]

17. Pan, P.; Yang, J.; Shan, G.; Bao, Y.; Weng, Z.; Inoue, Y. Nucleation effects of nucleobases on the crystallization kinetics of poly(L-lactide). Macromol. Mater. Eng. 2012, 297, 670-679. [CrossRef]

18. Bai, H.; Zhang, W.; Deng, H.; Zhang, Q.; Fu, Q. Control of crystal morphology in poly(L-lactide) by adding nucleating agent. Macromolecules 2011, 44, 1233-1237. [CrossRef]

19. Bai, H.; Huang, C.; Xiu, H.; Zhang, Q.; Fu, Q. Enhancing mechanical performance of polylactide by tailoring crystal morphology and lamellae orientation with the aid of nucleating agent. Polymer 2014, 55, 6924-6934. [CrossRef]

20. Ma, P.; Xu, Y.; Shen, T.; Dong, W.; Chen, M.; Lemstra, P.J. Tailoring the crystallization behavior of poly(L-lactide) with self-assembly-type oxalamide compounds as nucleators: 1 . Effect of terminal configuration of the nucleators. Eur. Polym. J. 2015, 70, 400-411. [CrossRef]

21. Ma, P.; Xu, Y.; Wang, D.; Dong, W.; Chen, M. Rapid crystallization of poly(lactic acid) by using tailor-made oxalamide derivatives as novel soluble-type nucleating agents. Ind. Eng. Chem. Res. 2014, 53, 12888-12892. [CrossRef]

22. Shen, T.; Xu, Y.; Cai, X.; Ma, P.; Dong, W.; Chen, M. Enhanced crystallization kinetics of poly(lactide) with oxalamide compounds as nucleators: Effect of spacer length between the oxalamide moieties. RSC Adv. 2016, 6, 48365-48374. [CrossRef]

23. Somani, R.H.; Yang, L.; Hsiao, B.S.; Sun, T.; Pogodina, N.V.; Lustiger, A. Shear-induced molecular orientation and crystallization in isotactic polypropylene: Effects of the deformation rate and strain. Macromolecules 2005, 38, 1244-1255. [CrossRef]

24. Kumaraswamy, G.; Kornfield, J.A.; Yeh, F.; Hsiao, B.S. Shear-enhanced crystallization in isotactic polypropylene. 3. Evidence for a kinetic pathway to nucleation. Macromolecules 2002, 35, 1762-1769. [CrossRef]

25. Hsiao, B.S.; Yang, L.; Somani, R.H.; Avila-Orta, C.A.; Zhu, L. Unexpected shish-kebab structure in a sheared polyethylene melt. Phys. Rev. Lett. 2005, 94, 117802. [CrossRef] [PubMed]

26. Somani, R.H.; Hsiao, B.S.; Nogales, A.; Srinivas, S.; Tsou, A.H.; Sics, I.; Balta-Calleja, F.J.; Ezquerra, T.A. Structure development during shear flow-induced crystallization of i-PP: In-situ small-angle X-ray scattering study. Macromolecules 2000, 33, 9385-9394. [CrossRef]

27. Peterlin, A. Drawing and extrusion of semi-crystalline polymers. Colloid Polym. Sci. 1987, 265, 357-382. [CrossRef]

28. Ghosh, S.; Viana, J.C.; Reis, R.L.; Mano, J.F. Effect of processing conditions on morphology and mechanical properties of injection-molded poly L-lactic acid. Polym. Eng. Sci. 2007, 47, 1141-1147. [CrossRef]

29. Yamazaki, S.; Itoh, M.; Oka, T.; Kimura, K. Formation and morphology of "shish-like" fibril crystals of aliphatic polyesters from the sheared melt. Eur. Polym. J. 2010, 46, 58-68. [CrossRef]

30. Tang, H.; Chen, J.B.; Wang, Y.; Xu, J.Z.; Hsiao, B.S.; Zhong, G.J.; Li, Z.M. Shear flow and carbon nanotubes synergistically induced nonisothermal crystallization of poly(lactic acid) and its application in injection molding. Biomacromolecules 2012, 13, 3858-3867. [CrossRef] [PubMed]

31. Tsuji, H. In vitro hydrolysis of blends from enantiomeric poly(lactide)s. Part 4: Well-homo-crystallized blend and nonblended films. Biomaterials 2003, 24, 537-547. [CrossRef]

32. Södergård, A.; Stolt, M. Properties of lactic acid based polymers and their correlation with composition. Prog. Polym. Sci. 2002, 27, 1123-1163. [CrossRef]

33. Saeidlou, S.; Huneault, M.A.; Li, H.; Park, C.B. Poly(lactic acid) crystallization. Prog. Polym. Sci. 2012, 37, 1657-1677. [CrossRef] 
34. Inkinen, S.; Hakkarainen, M.; Albertsson, A.C.; Södergård, A. From lactic acid to poly(lactic acid) (PLA): Characterization and analysis of PLA and its precursors. Biomacromolecules 2011, 12, 523-532. [CrossRef] [PubMed]

35. Pan, P.; Kai, W.; Zhu, B.; Dong, T.; Inoue, Y. Polymorphous crystallization and multiple melting behavior of poly(L-lactide): Molecular weight dependence. Macromolecules 2007, 40, 6898-6905. [CrossRef]

36. Zhang, J.M.; Duan, Y.X.; Sato, H.; Tsuji, H.; Noda, I.; Yan, S.; Ozaki, Y. Crystal modifications and thermal behavior of poly(L-lactic acid) revealed by infrared spectroscopy. Macromolecules 2005, 38, 8012-8021. [CrossRef]

37. Kawai, T.; Rahman, N.; Matsuba, G.; Nishida, K.; Kanaya, T.; Nakano, M.; Nakajima, K. Crystallization and melting behavior of poly(L-lactic acid). Macromolecules 2007, 40, 9463-9469. [CrossRef]

38. Puiggali, J.; Ikada, Y.; Tsuji, H.; Cartier, L.; Okihara, T.; Lotz, B. The frustrated structure of poly(L-lactide). Polymer 2000, 41, 8921-8930. [CrossRef]

39. Cartier, L.; Okihara, T.; Ikada, Y.; Tsuji, H.; Puiggali, J.; Lotz, B. Epitaxial crystallization and crystalline polymorphism of polylactides. Polymer 2000, 41, 8909-8919. [CrossRef]

40. Wei, X.; Bao, R.; Cao, Z.Q.; Yang, W.; Xie, B.; Yang, M. Stereocomplex crystallite network in asymmetric PLLA/PDLA blends: Formation, structure, and confining effect on the crystallization rate of homocrystallites. Macromolecules 2014, 47, 1439-1448. [CrossRef]

41. Luo, F.; Geng, C.; Wang, K.; Deng, H.; Chen, F.; Fu, Q.; Na, B. New understanding in tuning toughness of $\beta$-polypropylene: The role of $\beta$-nucleated crystalline morphology. Macromolecules 2009, 42, 9325-9331. [CrossRef]

42. Zhong, Y.; Fang, H.; Zhang, Y.; Wang, Z.; Yang, J.; Wang, Z. Rheologically determined critical shear rates for shear-induced nucleation rate enhancements of poly(lactic acid). ACS Sustain. Chem. Eng. 2013, 1, 663-672. [CrossRef]

43. Wasanasuk, K.; Tashiro, K. Crystal structure and disorder in Poly(L-lactic acid) $\delta$ form ( $\alpha^{\prime}$ form) and the phase transition mechanism to the ordered $\alpha$ form. Polymer 2011, 52, 6097-6109. [CrossRef]

44. Tsuji, H.; Tashiro, K.; Bouapao, L.; Hanesaka, M. Separate crystallization and cocrystallization of poly(L-lactide) in the presence of L-lactide-based copolymers with low crystallizability, poly(L-lactideco-glycolide) and poly(L-lactide-co-D-lactide). Macromol. Chem. Phys. 2012, 213, 2099-2112. [CrossRef]

45. Zhang, J.; Tashiro, K.; Tsuji, H.; Domb, A.J. Disorder-to-order phase transition and multiple melting behavior of poly(L-lactide) investigated by simultaneous measurements of WAXD and DSC. Macromolecules 2008, 41, 1352-1357. [CrossRef]

46. Zhang, H.; Bai, H.; Liu, Z.; Zhang, Q.; Fu, Q. Towards high-performance poly(L-lactide) fibers via tailoring crystallization with the aid of fibrillar nucleating agent. ACS Sustain. Chem. Eng. 2016, 4, 3939-3947. [CrossRef]

(C) 2016 by the authors. Licensee MDPI, Basel, Switzerland. This article is an open access article distributed under the terms and conditions of the Creative Commons Attribution (CC BY) license (http:/ / creativecommons.org/licenses/by/4.0/). 
Article

\title{
Investigation of Thermal and Thermomechanical Properties of Biodegradable PLA/PBSA Composites Processed via Supercritical Fluid-Assisted Foam Injection Molding
}

\author{
Sai Aditya Pradeep ${ }^{1,2}$, Hrishikesh Kharbas ${ }^{3}$, Lih-Sheng Turng ${ }^{3}$, Abraham Avalos ${ }^{4}$, \\ Joseph G. Lawrence ${ }^{4}$ and Srikanth Pilla ${ }^{1,2, *}$ \\ 1 Department of Automotive Engineering, Clemson University, Clemson, SC 29607, USA; \\ spradee@g.clemson.edu \\ 2 Department of Material Science and Engineering, Clemson University, Clemson, SC 29634, USA \\ 3 Polymer Engineering Center, Department of Mechanical Engineering, University of Wisconsin-Madison, \\ Madison, WI 53706, USA; kharbas@gmail.com (H.K.); turng@engr.wisc.edu (L.-S.T.) \\ 4 Polymer Institute, University of Toledo, Toledo, OH 43606, USA; Abraham.Avalos@utoledo.edu (A.A.); \\ joseph.lawrence@utoledo.edu (J.G.L.) \\ * Correspondence: spilla@clemson.edu; Tel.: +1-864-283-7216
}

Academic Editors: Alexander Böker and Frank Wiesbrock

Received: 29 November 2016; Accepted: 5 January 2017; Published: 9 January 2017

\begin{abstract}
Bio-based polymer foams have been gaining immense attention in recent years due to their positive contribution towards reducing the global carbon footprint, lightweighting, and enhancing sustainability. Currently, polylactic acid (PLA) remains the most abundant commercially consumed biopolymer, but suffers from major drawbacks such as slow crystallization rate and poor melt processability. However, blending of PLA with a secondary polymer would enhance the crystallization rate and the thermal properties based on their compatibility. This study investigates the physical and compatibilized blends of PLA/poly (butylene succinate-co-adipate) (PBSA) processed via supercritical fluid-assisted $(\mathrm{ScF})$ injection molding technology using nitrogen $\left(\mathrm{N}_{2}\right)$ as a facile physical blowing agent. Furthermore, this study aims at understanding the effect of blending and ScF foaming of PLA/PBSA on crystallinity, melting, and viscoelastic behavior. Results show that compatibilization, upon addition of triphenyl phosphite (TPP), led to an increase in molecular weight and a shift in melting temperature. Additionally, the glass transition temperature $\left(T_{\mathrm{g}}\right)$ obtained from the $\tan \delta$ curve was observed to be in agreement with the $T_{\mathrm{g}}$ value predicted by the Gordon-Taylor equation, further confirming the compatibility of PLA and PBSA. The compatibilization of ScF-foamed PLA-PBSA was found to have an increased crystallinity and storage modulus compared to their physically foamed counterparts.
\end{abstract}

Keywords: polylactide; poly(butylene succinate-co-adipate); compatibilization; crystallization; foaming

\section{Introduction}

Thermoplastic foams, as lightweight materials, are extensively used in sectors such as automotive, packaging, and aerospace due to advantages such as high strength-to-weight ratios, acoustic properties, low susceptibility to water vapor, superior impact resistance, and low densities [1]. However, a majority of these foams have precursors that are sourced from crude oil, which is a finite, non-renewable resource and a major cause of increasing carbon emissions that contribute to anthropogenic climate change. In the present paradigm, bio-based compostable thermoplastic foams have been gaining ground in many industries as they help to meet environmental regulations and standards set by international 
and domestic agencies via their application. Polylactic acid (PLA) is an aliphatic polyester that has emerged as one of the most commercially successful biopolymers due to its transparency, high strength, and high stiffness, making it superior to many other bio-based polymers [2-5]. However, despite its several advantages, commercially available PLA has many inherent weaknesses-in particular, its low toughness, low heat resistance, and brittleness - that prevent it from being widely adopted for durable applications. More importantly, PLA has poor melt processability due to a narrow processing window and a very slow crystallization rate. Typically, a higher crystallinity is desirable in finished products due to its strong influence on mechanical and thermal properties.

Several strategies exist to overcome the slow crystallization rate and melt processability of PLA, such as the addition of fillers [6,7], copolymerization [8,9], and melt-blending [10-12], all of which offer an effective medium for enhancing its overall performance. Among these, blending with inherently toughened (bio)polymers is one of the most effective solutions. However, most of these physical blends are immiscible in nature and can lead to the overall deterioration of properties [13]. The successful application of the reactive compatibilization technique has provided enormous opportunities to enhance the compatibility of blends that are otherwise immiscible and incompatible. Reactive compatibilization can be achieved via melt-blending of PLA with other suitable polymers, resulting in the formation of a block or graft copolymer at the interface and reducing the interfacial tension of immiscible polymer components, thereby promoting interfacial adhesion [13]. While blending PLA with toughened polymers enables us to overcome some of its aforementioned drawbacks, the foaming of PLA and its blends is critical to obtaining lightweight, sustainable thermoplastic foams.

In this context, supercritical fluid (ScF)-assisted injection molding, also known as microcellular injection molding, has been shown to broaden the processing window for biopolymers such as PLA, as it employs supercritical $\mathrm{N}_{2}$ or $\mathrm{CO}_{2}$ [14,15]. The broadening of the processing window is because ScF lowers the melt viscosity of the polymer [16,17] due to the formation of a single-phase polymer/gas solution, enabling the polymer to be processed at lower temperatures [18]. In addition ScF-assisted injection molding produces foamed components containing micron-sized cells and high cell densities while consuming a smaller amount of material and energy and having lower cycle times vs. conventional injection molding [19-21]. Hence, the production of lightweight, sustainable foams via eco-friendly processing routes necessitates an advanced understanding of the effects of reactive compatibilization on thermal and viscoelastic response of such foaming systems for enhancing their commercial application.

Several studies have been conducted on the melt-blending and foaming of PLA with secondary (bio)polymers, such as poly( $\varepsilon$-caprolactone) (PCL) [22], poly(hydroxybutyrate) (PHB) [23], polyhydroxybutyrate-valerate (PHBV) [24], poly(butylene succinate) (PBS) [25], poly(butylene adipate-co-terephthalate) (PBAT) [26], and poly(butylene succinate-co-adipate) (PBSA) [27]. Wu et al. [22] observed an increase in the crystallinity of compression-molded PLA-PCL blends upon incorporation of nanofillers. Abdelwahab et al. [23] reported improvement in crystalinity on compatibalized blends of PLA/PHB processed via compression-molding. Zhao et al. [24,28] prepared ScF-foamed physical blends of PLA/PHBV blends and composites, and studied their impact on crystallinity and thermomechanical properties. Yokohara and Yamaguchi [25] found that compression-molded PLA/PBS blends led to improved crystallinity and enhanced processability. Javadi et al. [26] studied the miscibility and the thermal and mechanical properties of ScF-foamed physical blends of PLA/PBAT and found improvements in damping ability. Ojijo et al. [27] studied compression-molded PLA/PBSA blends compatibilized via triphenyl phosphite (TPP) and observed a significant increase in crystallinity and thermal stability.

To the best of our knowledge, no studies have been undertaken on analyzing the effect of compatibilization and foaming on the crystallization and thermomechanical behavior of PLA/PBSA blends and composites processed via ScF injection molding. Hence, the objectives of this study are to understand the impact of compatibilization, $\mathrm{ScF}$ foaming, and the addition of talc on thermal behavior and viscoelastic properties. The (70:30) PLA/PBSA ratio was chosen as a model blend to study and 
understand the above-mentioned effects since it exhibited the highest crystallinity and improved properties, as detailed in Ojijo et al. [29].

\section{Materials and Methods}

Commercial polylactic acid (PLA) (3001 D) was purchased in pelletized form from Natureworks LLC (Minnetonka, MN, USA), with specific gravity of 1.24 and melt flow index of $\sim 22 \mathrm{~g} / 10 \mathrm{~min}$. Commercially available poly (butylene succinate-co-adipate) (PBSA) (Bionolle \#3001) pellets were sourced from Showa Denko (Tokyo, Japan), its specific gravity being 1.23 and melt flow index being $25 \mathrm{~g} / 10 \mathrm{~min}$. Talc used in this study (Mistrocell M90) was supplied by Imerys Talc (San Jose, CA, USA) with a mean diameter of $18.8 \mu \mathrm{m}$. Coupling agent triphenyl phosphite (TPP) was obtained from Sigma-Aldrich (Milwaukee, WI, USA), and 2 wt \% TPP was used to compatibilize blends. Commercial-grade nitrogen was sourced from Airgas (Greenville, SC, USA) and used as a blowing agent in ScF-assisted injection molding.

\subsection{Methods}

A co-rotating twin screw extruder (ZSK 30 from Werner \& Pfleiderer, Stuttgart, Baden-Württemberg, Germany) was used to compound the eight compositions prepared for this study, as listed in Table 1. Prior to extrusion, as-received PLA and PBSA pellets were dried at $75^{\circ} \mathrm{C}$ for $8 \mathrm{~h}$. Subsequently, talc and/or TPP were manually mixed with PLA/PBSA pellets in weight compositions listed in Table 1. Except for pure and talc-filled PBSA, extrusion for other compositions was subsequently carried out at temperature zones of $130 / 150 / 165 / 170 / 175{ }^{\circ} \mathrm{C}$ at a screw rotation speed of $35 \mathrm{rpm}$. Due to its low melting point $\left(91^{\circ} \mathrm{C}\right)$, extrusion of pure and talc-filled PBSA was carried out at temperatures of $100 / 125 / 135 / 140 / 145^{\circ} \mathrm{C}$.

Table 1. Design of experiment (DOE) formulations in this study (ratio) ${ }^{1}$.

\begin{tabular}{ccccc}
\hline Sample & Nomenclature & PLA & PBSA & Talc \\
\hline Pure PLA & A & 100 & - & - \\
Pure PBSA & B & - & 100 & - \\
Physical Blend & P & 70 & 30 & - \\
TPP Compatibilized blend & C & 70 & 30 & - \\
PLA + Talc & AT & 95 & - & 5 \\
PBSA + Talc & BT & - & 95 & 5 \\
Physical Blend + Talc & PT & 70 & 30 & 5 \\
TPP Compatibilized blend +Talc & CT & 70 & 30 & 5 \\
\hline
\end{tabular}

${ }^{1}$ Polylactic acid (PLA), poly(butylene succinate-co-adipate) (PBSA), triphenyl phosphite (TPP).

Extruded pellets of all compositions were dried at $80^{\circ} \mathrm{C}$ for $8 \mathrm{~h}$ prior to injection molding (IM). Conventional and ScF-assisted IM were carried out using an injection molding machine (Arburg Allrounder 3205, Lossburg, Baden-Württemberg, Germany), which was equipped with a Trexel Series II ScF dosing system, Wilmington, MA, USA. Injection molding parameters are listed in Table 2, while IM melt temperatures were reduced for pure PBSA to $100 / 140 / 145 / 135 / 125^{\circ} \mathrm{C}$ - similar to extrusion due to its low melting point. However, in the metering zone, temperatures had to be increased to ensure a consistent pressure drop during gas dosage. Weight $\%$ of supercritical $\mathrm{N}_{2}$ was calculated by Equation (1):

$$
\text { wt } \% \mathrm{ScF}=\frac{\dot{m} t X(27.8)}{m}
$$

where $\dot{m}$ is the mass flow rate of $\mathrm{ScF}_{\mathrm{c}}(\mathrm{kg} / \mathrm{h}), t$ is the $\mathrm{ScF}$ dosage time (s), $m$ is the shot weight $(\mathrm{g})$, and 27.8 is a conversion factor.

A total of 24 samples (solid IM and ScF IM with two gas dosages, 0.73 and 0.94 wt \%) -3 per each composition listed in Table 1 -were prepared. Subsequently, injection-molded specimens were labeled 
as "XX-Y", where $X X$ corresponds to nomenclature mentioned in Table 1 , while " $Y$ " indicates the nature of the sample as solid or foamed, with " $S$ " referring to the solid injection-molded sample, " 1 " referring to the ScF-assisted injection-molded sample obtained at a ScF gas dosage of $0.73 \mathrm{wt} \%$, and " 2 " referring to the ScF-assisted injection-molded sample at a gas dosage of $0.94 \mathrm{wt} \% \mathrm{ScF}$.

Table 2. Experimental conditions for solid and supercritical fluid assisted injection molding (ScF IM).

\begin{tabular}{ccc}
\hline Parameter & Solid Molding & Foamed Molding \\
\hline Back pressure $(\mathrm{MPa})$ & 10 & 80 \\
Melt temperatures $\left({ }^{\circ} \mathrm{C}\right)$ & $155 / 165 / 175 / 185 / 195$ & $155 / 165 / 175 / 185 / 195$ \\
Injection pressure $(\mathrm{bar})$ & 2500 & 2500 \\
Injection speed $\left(\mathrm{cm}^{3} / \mathrm{s}\right)$ & 65 & 65 \\
Holding pressure $(\mathrm{bar})$ & 800 & 0 \\
Holding time $(\mathrm{s})$ & 3 & 0 \\
Cooling time $(\mathrm{s})$ & 60 & 60 \\
Gas dosage $(\mathrm{wt} \%)$ & 0 & 0.73 and 0.94 \\
\hline
\end{tabular}

The IM samples were characterized using gel permeation chromatography (GPC), differential scanning calorimetry (DSC) and a dynamic mechanical analyzer (DMA) in order to understand the effect of physical and chemical compatibilization, the addition of fillers, and the ScF foaming of PLA and PBSA on thermal and viscoelastic properties.

\subsection{Gel Permeation Chromatography}

Number-average molecular weight $\left(M_{n}\right)$ and polydispersity index (PDI) for solid injection-molded samples were determined via gel permeation chromatography (GPC) on Waters GPC equipped with a UV-Vis and RI detector. Chloroform was used as an effluent (flow rate of $1.0 \mathrm{~mL} / \mathrm{min}$ ) at $33{ }^{\circ} \mathrm{C}$. All samples were prepared as $0.5 \%(w / v)$ solutions in chloroform, with $\sim 50 \mu \mathrm{L}$ of sample injected into the GPC. Prior to injection, the dissolved solution was filtered using a $0.2 \mu \mathrm{m}$ PTFE filter. Calibration was done using narrow molecular weight polystyrene standards ranging from $\sim 436$ to $\sim 990,500$ Daltons.

\subsection{Differential Scanning Calorimetry}

A differential scanning calorimeter (TA Instruments, Q2000, New Castle, DE, USA) was used to study the crystallization behavior of all 24 samples. About 7-9 $\mathrm{mg}$ of sample was taken in hermetically sealed aluminum pans. Samples were subjected to heating/cooling/heating cycles at $5{ }^{\circ} \mathrm{C} / \mathrm{min}$, beginning with heating from -100 to $200{ }^{\circ} \mathrm{C}$ (to remove any thermal history from processing), held isothermally for $5 \mathrm{~min}$, cooled to $-100{ }^{\circ} \mathrm{C}$, and subsequently heated to $200{ }^{\circ} \mathrm{C}$. The temperature of cold crystallization $\left(T_{\mathrm{cc}}\right)$, the melting temperature $\left(T_{\mathrm{m}}\right)$, the apparent melting enthalpy $\left(\Delta H_{\mathrm{m}}\right)$, and the enthalpy of cold crystallization $\left(\Delta H_{\mathrm{cc}}\right)$ were determined via DSC curves. The crystallinity of PLA and PBSA were calculated by Equation (2):

$$
\chi_{\mathrm{C}}(\% \text { crystallinity })=\frac{\Delta H_{\mathrm{m}}-\Delta H_{\mathrm{cc}}}{\Delta H^{0}} \times \frac{100}{W}
$$

where $\Delta H_{\mathrm{m}}(\mathrm{PLA})$ and $\Delta H_{\mathrm{m}}$ (PBSA) are the enthalpies of melting per gram of $100 \%$ crystal (perfect crystal) of PLA and PBSA (93.7 and $142 \mathrm{~J} / \mathrm{g}$ ), respectively, and $W$ is the weight fraction of either PLA or PBSA in the blend [30,31].

\subsection{Dynamic Mechanical Analyzer}

Dynamic mechanical analysis was carried out using TA Q800 Dynamic Mechanical Analyzer, New Castle, DE, USA. Rectangular specimen $(4 \mathrm{~mm} \times 8 \mathrm{~mm} \times 70 \mathrm{~mm})$ were cut from the gauge length of injection-molded specimen and tested in dual cantilever mode. Samples were tested at temperatures between -50 and $100{ }^{\circ} \mathrm{C}$ at a heating rate of $3{ }^{\circ} \mathrm{C} / \mathrm{min}$ at a $1 \mathrm{~Hz}$ frequency and a $0.1 \%$ strain amplitude in order to determine glass transition temperature, storage, and loss moduli. 


\section{Results}

\subsection{Gel Permeation Chromatography}

Number-average molecular weight $\left(M_{n}\right)$, weight-average molecular weight $\left(M_{W}\right)$, and polydispersity index (PDI) were determined for all solid samples and tabulated in Table 3. As can be seen,

(a) $\quad M_{n}$ of PLA (A-S) and PBSA (B-S) were obtained as $\sim 90,000$ and $\sim 62,000$ Daltons, respectively;

(b) While the $M_{n}$ of the physical blends (P-S) ( 64,000 Daltons) was found to be between that of PLA (A-S) and PBSA (B-S), compatibilized blends (C-S) showed a higher $M_{\mathrm{n}}(\sim 101,796$ Daltons) compared to both P-S (by over $\sim 40,000$ Daltons) and A-S (by over $\sim 10,000$ Daltons);

(c) Addition of talc resulted in a marginal reduction in the $M_{n}$ of PLA (AT-S) and a marginal increase in the $M_{n}$ of all other compositions-namely, BT-S, PT-S, and CT-S-compared to its non-talc counterparts-within talc-filled compositions, the $M_{\mathrm{n}}$ of PT-S ( 79,026 Daltons) was found to be between those of AT-S ( 85,083 Daltons) and BT-S ( 66,173 Daltons), while CT-S ( 108,483 Daltons) showed an improvement over all three compositions;

(d) PDI for pure and talc-filled compatibalized blends (C-S and CT-S) was found to be narrower than that for other compositions.

Table 3. $M_{\mathrm{n}}, M_{\mathrm{W}}$, polydispersity index (PDI), and area for all compositions.

\begin{tabular}{ccc}
\hline Sample & $\boldsymbol{M}_{\mathbf{n}}$ (Daltons) & PDI \\
\hline A-S & 90,039 & 1.8 \\
B-S & 62,175 & 2.1 \\
P-S & 64,685 & 2.1 \\
C-S & 101,796 & 1.4 \\
AT-S & 85,083 & 1.7 \\
BT-S & 66,173 & 2.0 \\
PT-S & 79,026 & 1.8 \\
CT-S & 108,483 & 1.4 \\
\hline
\end{tabular}

\subsection{Differential Scanning Calorimetry}

\subsubsection{First Heating Thermograms}

Temperature of cold crystallization $\left(T_{\mathrm{cc}}\right)$, melting temperature $\left(T_{\mathrm{m}}\right)$, and their respective ethalpies of cold crystalization $\left(\Delta H_{\mathrm{cc}}\right)$ and $\left(\Delta H_{\mathrm{m}}\right)$-as obtained from first heating thermograms-are reported for pure polymer (Table 4 ) and for polymer blends (Table 5), respectively.

Table 4. The thermal behavior of injection-molded pure samples obtained from 1st heating thermograms.

\begin{tabular}{cccccc}
\hline Sample & $\boldsymbol{T}_{\mathrm{cc}}\left({ }^{\circ} \mathrm{C}\right)$ & $\Delta \boldsymbol{H}_{\mathrm{cc}}(\mathrm{J} / \mathrm{g})$ & $\boldsymbol{T}_{\mathrm{m}}\left({ }^{\circ} \mathrm{C}\right)$ & $\Delta \boldsymbol{H}_{\mathrm{m}}(\mathrm{J} / \mathrm{g})$ & $\%$ Crystallinity \\
\hline A-S & 97.3 & 26.42 & 168.8 & 45.34 & 20.19 \\
A-1 & 99.6 & 26 & 168.7 & 46 & 21.34 \\
A-2 & 100.8 & 22.41 & 168.7 & 47.76 & 27.05 \\
AT-S & 90.2 & 19.23 & 168.4 & 43.51 & 25.91 \\
AT-1 & 90.9 & 17.63 & 168.2 & 43.04 & 27.11 \\
AT-2 & 91.4 & 18.19 & 168.2 & 44.22 & 27.78 \\
B-S & - & - & 92.9 & 47.22 & 33.25 \\
B-1 & - & - & 92.9 & 49.99 & 35.20 \\
B-2 & - & - & 92.9 & 50.75 & 35.73 \\
BT-S & - & - & 93.0 & 49 & 34.50 \\
BT-1 & - & - & 92.8 & 51 & 35.91 \\
BT-2 & - & 92.9 & 52 & 36.61 \\
\hline
\end{tabular}

Among individual polymer compositions, pure PLA compositions showed $T_{\mathrm{cc}}$ values of $\sim 97.3^{\circ} \mathrm{C}$ (A-S), $\sim 99.6^{\circ} \mathrm{C}(\mathrm{A}-1)$, and $\sim 100{ }^{\circ} \mathrm{C}(\mathrm{A}-2)$, respectively. Compared to the physical blends $\left(T_{\mathrm{cc}} \sim 81^{\circ} \mathrm{C}\right)$, 
chemically compatibilized blends exhibited a significant reduction in $T_{\mathrm{cc}}\left(T_{\mathrm{cc}} \sim 71^{\circ} \mathrm{C}\right)$. The introduction of talc in the PLA samples led to a reduction in $T_{\mathrm{cc}}$ compared to pure PLA compositions, such as from $\sim 97.3{ }^{\circ} \mathrm{C}$ (A-S) to $\sim 90.2{ }^{\circ} \mathrm{C}$ (AT-S) or from $\sim 100{ }^{\circ} \mathrm{C}$ (A-2) to $\sim 91.4{ }^{\circ} \mathrm{C}$ (AT-2), respectively. In the case of physical and chemically compatibilized PLA-PBSA blends, the addition of talc did not significantly alter $T_{\mathrm{CC}}$ compared to non-talc counterparts. All $T_{\mathrm{CC}}$ values observed in the blended samples correspond to the PLA component, while PBSA samples (both talc-filled and non-talc) did not exhibit any $T_{\mathrm{cc}}$ value.

With regard to melting behavior, while all PLA samples showed a single melting peak at $\sim 168{ }^{\circ} \mathrm{C}$, all PBSA samples showed a single melting peak at $\sim 92^{\circ} \mathrm{C}$. However, in the case of blended samples, two melting peaks were observed, one each corresponding to melting temperatures of PBSA and PLA, respectively. While physically blended samples showed melting peaks at $\sim 92$ and $\sim 167^{\circ} \mathrm{C}$, chemically blended samples showed a shift in both melting peaks to $\sim 88$ and $\sim 155^{\circ} \mathrm{C}$. The addition of talc and/or $\mathrm{ScF}$ was not found to result in any significant shift in melting point $\left(T_{\mathrm{m}}\right)$.

With respect to crystallinity, solid PLA (A-S) exhibited a crystallinity of $\sim 20.19 \%$, while its foamed counterpart (A-2) showed a higher crystallinity of $\sim 27 \%$. A similar increase in crystallinity of PBSA was observed from $\sim 33 \%$ (B-S) to $\sim 35 \%$ (B-1 and B-2). While physically blended foamed samples (P-1, P-2, PT-1, and PT-2) showed a crystallinity of $\sim 15 \%-18 \%$, their chemically conjugated foamed counterparts (C-1, C-2, CT-1, and CT-2) exhibited crystallinity levels of $\sim 20 \%-28 \%$. The addition of talc was observed to improve crystallinity to varying degrees for all compositions compared to their non-talc counterparts, both for solid and foamed compositions. For example, while AT-S showed improvement in crystallinity by $\sim 5 \%$ compared to A-S, physical blends showed improvement by $2 \%$ compared to their non-talc counterparts.

Table 5. Thermal behavior of injection-molded blends obtained from 1st heating thermograms.

\begin{tabular}{ccccccc}
\hline Sample & $\boldsymbol{T}_{\mathrm{cc}}\left({ }^{\circ} \mathrm{C}\right)$ & $\Delta \boldsymbol{H}_{\mathrm{cc}}(\mathrm{J} / \mathrm{g})$ & $\boldsymbol{T}_{\mathrm{m} 1}^{\mathrm{PBSA}}\left({ }^{\circ} \mathrm{C}\right)$ & $\boldsymbol{T}_{\mathbf{m} 2}^{\mathrm{PLA}}\left({ }^{\circ} \mathrm{C}\right)$ & $\Delta \boldsymbol{H}_{\mathrm{m}}(\mathrm{J} / \mathrm{g})$ & $\%$ Crystallinity \\
\hline P-S & 81.2 & 21.97 & 92.8 & 167.8 & 32.14 & 15.50 \\
P-1 & 81.1 & 21.07 & 92.1 & 167.8 & 32.03 & 16.70 \\
P-2 & 81.0 & 20.97 & 92.5 & 167.9 & 31.62 & 16.23 \\
PT-S & 81.1 & 18.5 & 92.8 & 167.8 & 29.85 & 17.30 \\
PT-1 & 81.0 & 16.29 & 93.0 & 167.8 & 28.23 & 18.20 \\
PT-2 & 80.9 & 16.48 & 93.1 & 167.5 & 29.19 & 19.37 \\
C-S & 71.3 & 25.33 & 88.0 & 155.6 & 35.83 & 16.00 \\
C-1 & 71.4 & 20.868 & 88.6 & 158.0 & 33.29 & 18.93 \\
C-2 & 71.0 & 18.04 & 88.1 & 155.5 & 34.07 & 24.40 \\
CT-S & 72.1 & 22 & 89.0 & 156.8 & 33 & 16.77 \\
CT-1 & 73.8 & 14.27 & 88.7 & 154.8 & 33.18 & 28.83 \\
CT-2 & 74.2 & 16.7 & 89.0 & 155.2 & 34.27 & 26.78 \\
\hline
\end{tabular}

\subsubsection{Second Heating Thermograms}

The second heating thermograms for all samples is shown in Figure 1a-d, while the glass transition temperature $\left(T_{\mathrm{g}}\right)$, the melting temperature $\left(T_{\mathrm{m}}\right)$, and crystallinity levels $(\%)$ obtained from these thermograms are reported for the pure polymers (Table 6) and for the blends (Table 7).

$T_{\mathrm{g}}$ for solid PLA (A-S) was observed to be $63.8^{\circ} \mathrm{C}$ with marginal decrease for both foamed compositions (A-1 and A-2). A similar trend was observed for PBSA, with $T_{\mathrm{g}}$ gradually reducing from $-41.8^{\circ} \mathrm{C}$ (solid PBSA or B-S) to lower values for both foamed counterparts (B-1 and B-2). However, $T_{g}$ for both physical and chemical blends could not be observed at the ramp rate tested in this study.

Melting point $\left(T_{\mathrm{m}}\right)$ was observed to be $169^{\circ} \mathrm{C}$ for all pure PLA compositions (A-S, A-1, and A-2), albeit with the addition of talc (AT-S, AT-1, and AT-2) resulting in obtainment of bimodal melting peaks at $\sim 165$ and $\sim 171{ }^{\circ} \mathrm{C}$, respectively. However, PBSA showed a consistent single melting peak of $\sim 92{ }^{\circ} \mathrm{C}$ for all PBSA samples (both talc-filled and non-talc). Physically blended solid samples showed three melting peaks: one at $\sim 94.6{ }^{\circ} \mathrm{C}$ (corresponding to PBSA), and bimodal peaks at $\sim 165$ and $\sim 170{ }^{\circ} \mathrm{C}$ (corresponding to PLA). Chemically compatibilized solid samples exhibited a similar trend, with melting peaks at $\sim 90.0^{\circ} \mathrm{C}$ (corresponding to PBSA), and bimodal peaks at $\sim 158.8$ and 164.1 
${ }^{\circ} \mathrm{C}$ (corresponding to PLA). Interestingly, their foamed counterparts (P-1, P-2, C-1, and C-2) showed only two peaks at $\sim 93^{\circ} \mathrm{C}$ (corresponding to PBSA) and $\sim 169^{\circ} \mathrm{C}$ (a single peak corresponding to PLA). The addition of talc to blends resulted in the obtainment of bimodal peaks (corresponding to PLA) in physical blends (PT-S, PT-1, and PT-2), in stark contrast to a single melting peak (corresponding to PLA) in chemical blends (CT-S, CT-1, and CT-2).

With regard to crystallinity, the addition of talc led to an increase in crystallinity of PLA and PBSA samples by $\sim 4 \%$ and $\sim 5 \%$, respectively. The crystallinity of the PLA component in the blends was observed to enhance by $\sim 4 \%$ for both physical and chemically compatibilized blends, with the effect of talc being more pronounced for compatibilized blends. Chemically compatibilized foamed blends (C-1, C-2, CT-1, and CT-2) showed higher crystallinity vs. their physically foamed counterparts (P-1, P-2, PT-1, and PT-2, respectively). However, the enhancement in crystallinity due to the use of ScF foaming was not as pronounced as that due to the addition of talc, with $\sim 50 \%$ crystallinity observed for talc-filled chemically compatibilized samples (CT-S, CT-1, and CT-2).

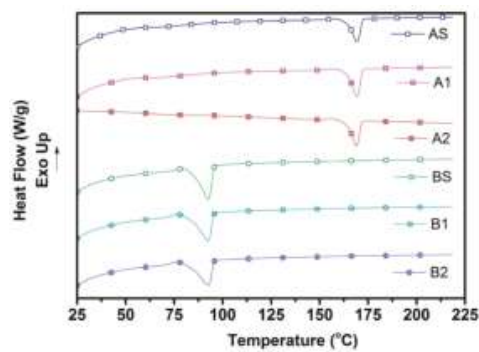

(a)

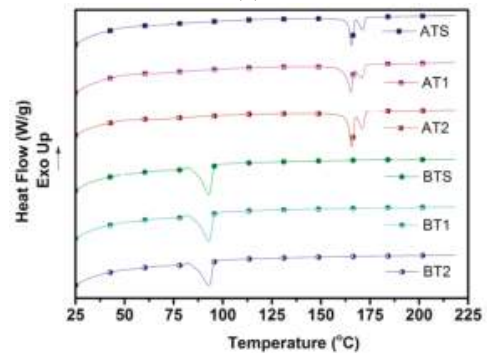

(c)

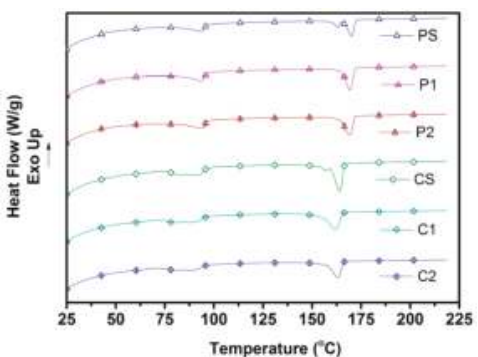

(b)

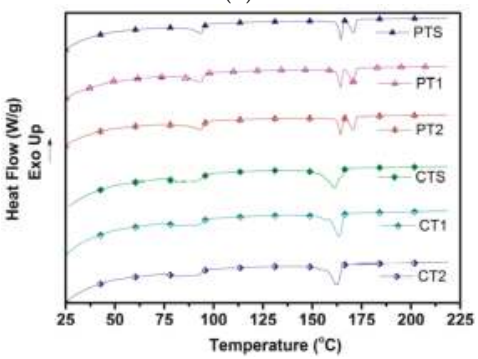

(d)

Figure 1. Second heating Differential Scanning Calorimetry (DSC) curves of (a) non-talc pure; (b) non-talc blend; (c) talc pure; and (d) talc blend compositions.

Table 6. Thermal properties of injection-molded samples obtained in 2nd heating thermograms.

\begin{tabular}{|c|c|c|c|c|c|c|c|c|}
\hline \multirow{2}{*}{ Sample } & \multirow{2}{*}{$T_{\mathrm{g}}\left({ }^{\circ} \mathrm{C}\right)$} & \multirow{2}{*}{$T_{\mathrm{m}}^{\mathrm{PBSA}}\left({ }^{\circ} \mathrm{C}\right)$} & \multicolumn{2}{|c|}{$T_{\mathrm{m}}^{\mathrm{PLA}}$} & \multirow{2}{*}{$\begin{array}{c}\Delta H_{\mathrm{m}} \text { of } \\
\text { PBSA }(\mathrm{J} / \mathrm{g})\end{array}$} & \multirow{2}{*}{$\begin{array}{c}\Delta H_{\mathrm{m}} \text { of } \\
\operatorname{PLA}(\mathrm{J} / \mathrm{g})\end{array}$} & \multirow{2}{*}{$\begin{array}{c}\% \text { Crystallinity } \\
\text { PBSA }\end{array}$} & \multirow{2}{*}{$\begin{array}{c}\% \text { Crystallinity } \\
\text { PLA }\end{array}$} \\
\hline & & & $T_{\mathrm{m} 1}\left({ }^{\circ} \mathrm{C}\right)$ & $T_{\mathrm{m} 2}\left({ }^{\circ} \mathrm{C}\right)$ & & & & \\
\hline A-S & 63.8 & - & 169.4 & - & - & 36.69 & - & 39.15 \\
\hline A-1 & 63.1 & - & 169.1 & - & - & 39.17 & - & 41.8 \\
\hline A-2 & 61.9 & - & 168.8 & - & - & 38.78 & - & 41.3 \\
\hline AT-S & 63.2 & - & 165.6 & 171.5 & - & 40.84 & - & 43.58 \\
\hline AT-1 & 63.1 & - & 165.1 & 170.6 & - & 43.01 & - & 46.90 \\
\hline AT-2 & 62.7 & - & 165.6 & 171.2 & - & 44.35 & - & 47.33 \\
\hline B-S & -41.8 & 92.7 & - & - & 37.24 & - & 26.22 & - \\
\hline B-1 & -42.8 & 92.4 & - & - & 43.16 & - & 30.39 & - \\
\hline B-2 & -42.1 & 92.7 & - & - & 44.37 & - & 31.24 & - \\
\hline BT-S & -42.3 & - & - & - & 45.4 & - & 31.97 & - \\
\hline BT-1 & -43.4 & - & - & - & 53.06 & - & 37.36 & - \\
\hline BT-2 & -43.0 & - & - & - & 54.29 & - & 38.23 & - \\
\hline
\end{tabular}


Table 7. Thermal properties of injection-molded samples obtained in 2nd heating thermograms.

\begin{tabular}{|c|c|c|c|c|c|c|c|}
\hline \multirow{2}{*}{ Sample } & \multirow{2}{*}{$T_{\mathrm{m}}^{\mathrm{PBSA}}\left({ }^{\circ} \mathrm{C}\right)$} & \multicolumn{2}{|c|}{$T_{\mathrm{m}}^{\mathrm{PLA}}$} & \multirow{2}{*}{$\begin{array}{c}\Delta H_{\mathrm{m}} \text { of } \\
\operatorname{PBSA}(\mathrm{J} / \mathrm{g})\end{array}$} & \multirow{2}{*}{$\begin{array}{c}\% \text { Crystallinity } \\
\text { PBSA }\end{array}$} & \multirow{2}{*}{$\begin{array}{c}\Delta H_{\mathrm{m}} \text { of } \\
\text { PLA }(\mathrm{J} / \mathrm{g})\end{array}$} & \multirow{2}{*}{$\begin{array}{c}\% \text { Crystallinity } \\
\text { PLA }\end{array}$} \\
\hline & & $T_{\mathrm{m} 1}\left({ }^{\circ} \mathrm{C}\right)$ & $T_{\mathrm{m} 2}\left({ }^{\circ} \mathrm{C}\right)$ & & & & \\
\hline P-S & 94.65 & 165.6 & 170.10 & 15.46 & 36.6 & 28.53 & 43.49 \\
\hline P-1 & 93.54 & 169.33 & - & 12.66 & 23.80 & 25.11 & 38.28 \\
\hline P-2 & 93.63 & 169.23 & - & 13.45 & 24.47 & 25.21 & 38.43 \\
\hline PT-S & 93.45 & 164.48 & 170.62 & 13.17 & 30.91 & 27.74 & 40.29 \\
\hline PT-1 & 93.25 & 164.20 & 170.06 & 13.76 & 32.30 & 27.90 & 42.53 \\
\hline PT-2 & 93.29 & 164.40 & 171.06 & 14.64 & 34.36 & 26.83 & 42.90 \\
\hline C-S & 90.03 & 158.81 & 164.14 & 11.13 & 34.51 & 34.51 & 42.90 \\
\hline C-1 & 87.75 & 160.39 & - & 9.39 & 22.042 & 30.39 & 46.33 \\
\hline C-2 & 88.61 & 160.97 & - & 10.67 & 25.04 & 30.74 & 46.86 \\
\hline CT-S & 90.67 & 160.23 & - & 9.916 & 23.27 & 35.76 & 54.52 \\
\hline CT-1 & 90.96 & 163.54 & - & 9.961 & 23.38 & 34.86 & 53.14 \\
\hline CT-2 & 90.28 & 162.71 & - & 9.54 & 22.39 & 34.49 & 52.58 \\
\hline
\end{tabular}

\subsection{Dynamic Mechanical Analysis}

Viscoelastic behavior of all samples was studied using DMA to track temperature dependence of storage modulus and $\tan \delta$. Figure $2 \mathrm{a}-\mathrm{d}$ show storage modulus curves as a function of temperature, while Figure 3a-d show dependence of $\tan \delta$ on temperature. All samples exhibited a decline in storage modulus with an increase in temperature. While a plateau region was observed for all PLA samples up to its $T_{\mathrm{g}}$ of $\sim 63{ }^{\circ} \mathrm{C}$, similar plateau regions were not observed for the blends and PBSA samples. Solid and foamed physical blends (P-S, P-1, and P-2) exhibited glass transition at $\sim 62{ }^{\circ} \mathrm{C}$ (corresponding to PLA), while compatibilized blends (C-S, C-1, and C-2) exhibited a shift in $T_{\mathrm{g}}$ to $\sim 53^{\circ} \mathrm{C}$ (corresponding to PLA). $T_{\mathrm{g}}$ values for all PBSA samples (B-S, B-1, B-2, BT-S, BT-1, and BT-2) was the same as that of PBSA at $\sim-40^{\circ} \mathrm{C}$.

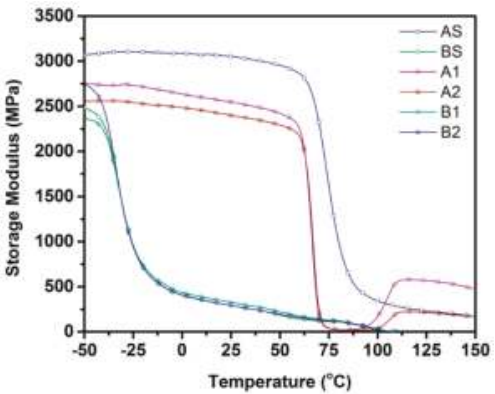

(a)

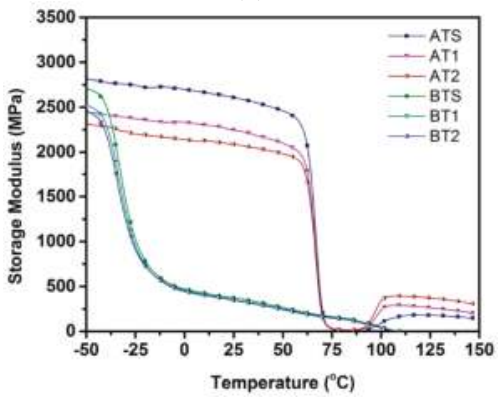

(c)

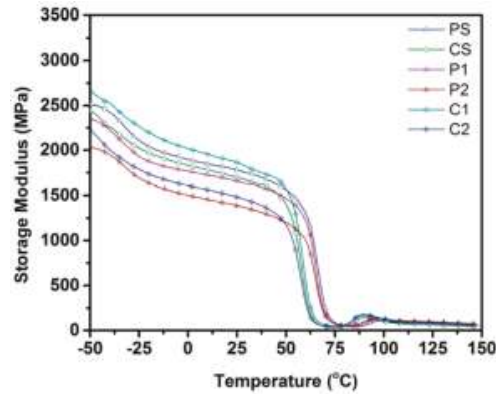

(b)

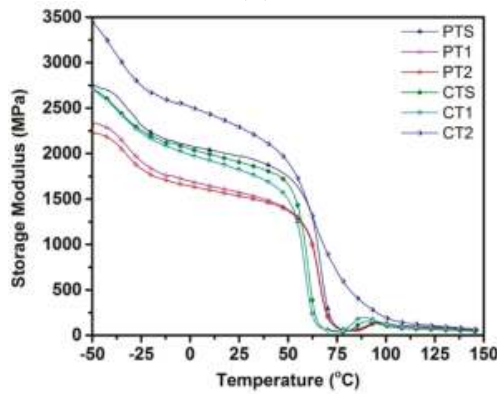

(d)

Figure 2. Storage modulus of (a) non-talc pure; (b) non-talc blend; (c) talc pure; and (d) talc blend compositions. 


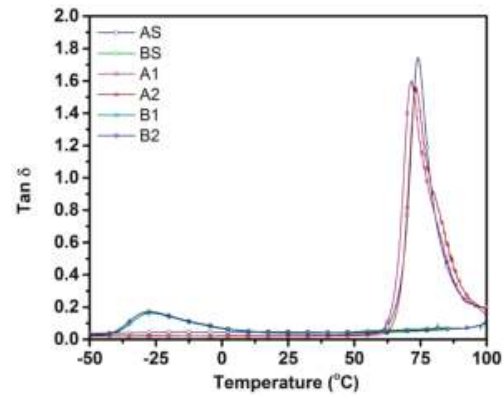

(a)

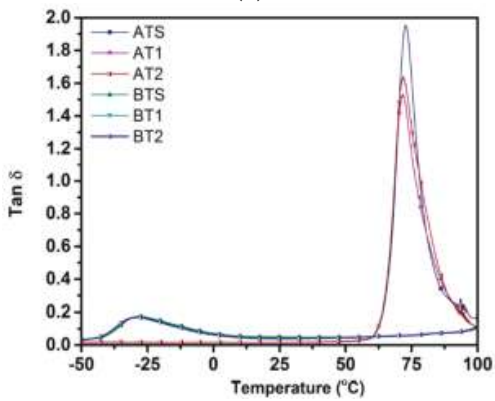

(c)

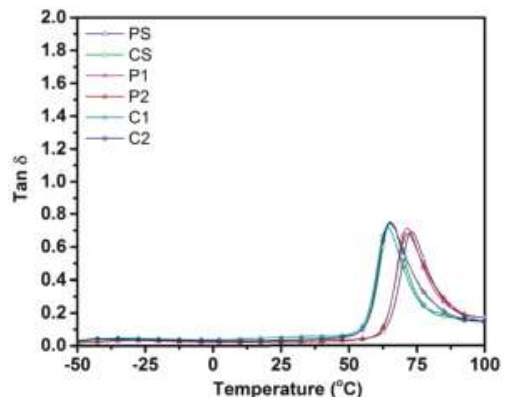

(b)

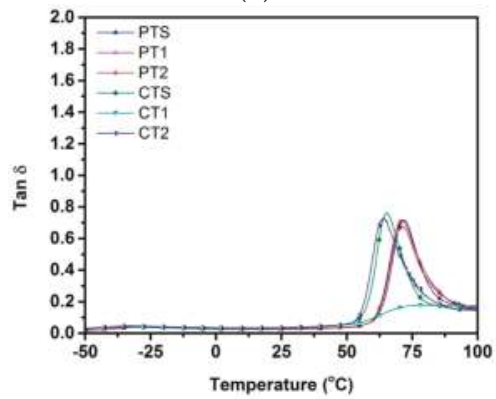

(d)

Figure 3. Tan $\delta$ of (a) non-talc pure; (b) non-talc blend; (c) talc pure; and (d) talc blend, compositions.

The storage moduli at -50 and $25{ }^{\circ} \mathrm{C}$ for all compositions is reported in Table 8 . The storage modulus at $-50{ }^{\circ} \mathrm{C}$ was observed to reduce upon the use of ScF for both talc-filled and non-talc PLA and PBSA samples, with non-talc-based PLA compositions showing a higher storage modulus vs. non-talc-filled PBSA or blend compositions (Figure 2a,b). Figure 2c,d shows that, in sum, talc-filled samples (excluding those of PLA) exhibited a higher storage modulus compared to their non-talc counterparts. While solid physical and chemically compatibilized blends exhibited distinct storage moduli of $\sim 2500 \mathrm{MPa}$ at $-50^{\circ} \mathrm{C}$, microcellular physical blends showed lower storage moduli (2315 and $2028 \mathrm{MPa}$ ), while chemically foamed blends showed higher storage moduli. Among foamed compatibilized blends, non-talc blends at a lower ScF gas dosage (C-1) exhibited the highest storage modulus among all non-talc blends, while CT-2 showed the highest storage modulus among all 24 samples.

Table 8. Storage moduli of all compositions at -50 and $25^{\circ} \mathrm{C}$.

\begin{tabular}{ccccccc}
\hline \multirow{2}{*}{ Sample } & \multicolumn{2}{c}{ Storage Modulus $\mathbf{( M P a )}$ at $\mathbf{- 5 0}{ }^{\circ} \mathbf{C}$} & \multicolumn{2}{c}{ Storage Modulus (MPa) at $\mathbf{2 5}{ }^{\circ} \mathbf{C}$} \\
\cline { 2 - 6 } & Solid & ScF $\mathbf{1}$ & ScF $\mathbf{2}$ & Solid & ScF 1 & ScF 2 \\
\hline A & 3069 & 2731 & 2555 & 3063 & 2552 & 2403 \\
B & 2500 & 2365 & 2650 & 292 & 336 & 295 \\
P & 2510 & 2315 & 2028 & 1776 & 1646 & 1386 \\
C & 2415 & 2653 & 2184 & 1709 & 1867 & 1478 \\
AT & 2812 & 2485 & 2304 & 2611 & 2245 & 2086 \\
BT & 2697 & 2507 & 2306 & 408 & 355 & 321 \\
PT & 2746 & 2332 & 2250 & 1972 & 1578 & 1535 \\
CT & 2685 & 2685 & 3429 & 1911 & 1826 & 2300 \\
\hline
\end{tabular}


The post-glass transition hump observed in Figure $2 \mathrm{a}-\mathrm{d}$ is analogous to a cold crystallization temperature $\left(T_{\mathrm{cc}}\right)$ [32]. While solid PLA samples did not show any $T_{\mathrm{cc}}$, the foamed PLA samples (A-1 and A-2) exhibited a $T_{\mathrm{CC}}$ of $\sim 108$ and $\sim 109{ }^{\circ} \mathrm{C}$, respectively. In contrast, no PBSA sample showed any $T_{\text {cc }}$. With regard to the non-talc-filled blend samples, the physically blended samples showed a $T_{\text {cc }}$ at $\sim 96{ }^{\circ} \mathrm{C}$, while chemically compatibilized blends exhibited a lower $T_{\mathrm{cc}}$ at $\sim 89{ }^{\circ} \mathrm{C}$. The addition of talc was observed to lead to a reduction in $T_{\mathrm{cc}}$ for the PLA samples (AT-1 and AT-2) to 101 and $\sim 102{ }^{\circ} \mathrm{C}$, respectively, with a reduction in $T_{\mathrm{cc}}$ for the physically blended samples $\left(\sim 95^{\circ} \mathrm{C}\right)$ and chemically compatibilized samples $\left(\sim 85^{\circ} \mathrm{C}\right)$. Interestingly, CT-2 did not show any cold crystallization temperature.

Tan $\delta$ is the ratio of loss modulus to storage modulus. Table 9 tabulates the glass transition temperature $\left(T_{\mathrm{g}}\right)$ corresponding to $\tan \delta$ peaks - as this is often analogous to $T_{\mathrm{g}}$ of the polymer-and the area under the tan $\delta$ curve. As shown in Figure $3 \mathrm{a}$, the $T_{\mathrm{g}}$ of the PLA is $\sim 75^{\circ} \mathrm{C}$; however, with chemical compatibilization, it was observed to undergo a significant shift to lower temperatures $\left(\sim 65^{\circ} \mathrm{C}\right)$ (Figure $\left.3 \mathrm{~b}\right)$. While the blend compositions showed no $T_{\mathrm{g}}$ corresponding to PBSA, the physical blends exhibited a $T_{\mathrm{g}}$ of $\sim 72{ }^{\circ} \mathrm{C}$, and compatibilized blends exhibited a relatively low $T_{\mathrm{g}}\left(\sim 64{ }^{\circ} \mathrm{C}\right)$, both corresponding to the $T_{\mathrm{g}}$ of the PLA. The addition of talc was not found to result in any significant shift in $T_{\mathrm{g}}$ of any composition based on their tan $\delta$ peaks, while the area under the $\tan \delta$ curve was observed to reduce for both physical and chemically compatibilized blends compared to the pure PLA-based compositions.

Table 9. Glass transition temperatures and area under tan $\delta$ for all compositions.

\begin{tabular}{ccccccc}
\hline \multirow{2}{*}{ Sample } & \multicolumn{3}{c}{$T_{\mathbf{g}}\left({ }^{\circ} \mathbf{C}\right)$} & \multicolumn{3}{c}{ Area under $\tan \delta$} \\
\cline { 2 - 7 } & Solid & ScF 1 & ScF 2 & Solid & ScF 1 & ScF 2 \\
\hline A & 75.1 & 71.1 & 71.2 & 27.3 & 26.1 & 24.9 \\
B & -27.4 & -27.55 & -27.35 & 9 & 9.9 & 10.9 \\
P & 72.9 & 71.7 & 71.8 & 11.6 & 12.17 & 12.35 \\
C & 64.5 & 64 & 63.7 & 12 & 13.5 & 13.4 \\
AT & 72 & 72.5 & 72.3 & 26.4 & 23.2 & 25.3 \\
BT & -28.3 & -28.7 & -28.9 & 8.8 & 8.3 & 8.1 \\
PT & 71.2 & 71.4 & 71.8 & 12.48 & 12.9 & 12.9 \\
CT & 64.2 & 64 & 64 & 13.7 & 8.1 & 14.4 \\
\hline
\end{tabular}

\section{Discussion}

\subsection{Compatibilization Mechanism}

Most physical blends of PLA with toughened secondary polymers (including PBSA) are thermodynamically immiscble [29]. It is common practice to add compatibilizers in order to improve the compatibility of these immiscible blends. An addition of compatibilizer results in a reduction of interfacial tension due to the formation of either a block or graft copolymer at interfaces within the blend, depending on the kind of compatibilizer used [13,33]. For example, an addition of compatibilizers possessing reactive end groups will result in the formation of block copolymers (with a substantial increase in $M_{n}$ ) [34], while an addition of compatibilizers with reactive pendant groups (such as TPP) will generally result in the formation of graft/branched copolymers [35].

Different researchers have undertaken studies on the effect of compatibilizers with reactive pendant groups (such as TPP) on polyester-based systems and have proposed two reaction mechanisms-one by Jacues et al. [35] and the other by Aharoni et al. [36]. These reaction mechanisms have a strong impact on the compatibilization of polymer blends and their properties. Hence, any understanding of how the addition of TPP influences the compatibilization of PLA and PBSA in this study needs to be taken into account. In both of the above-mentioned reaction mechanisms, the first step is the preferential reaction of hydroxyl end-groups of PLA/PBSA with TPP via the displacement of one of TPP's phenoxy groups, as shown in Figure 4a. This leads to the formation of an intermediate 
phosphorus-containing compound (intermediate alkyl diphenyl phosphite). The second step can be either of the two reaction mechanisms depicted in Figure $4 \mathrm{~b}$,c.

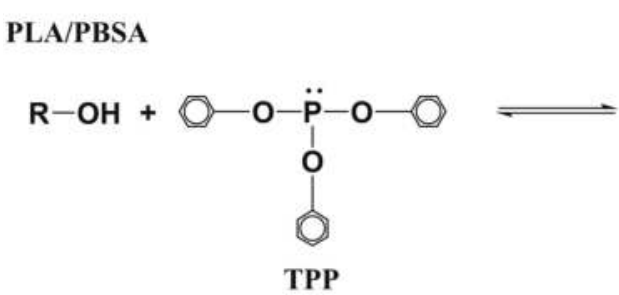

(a)
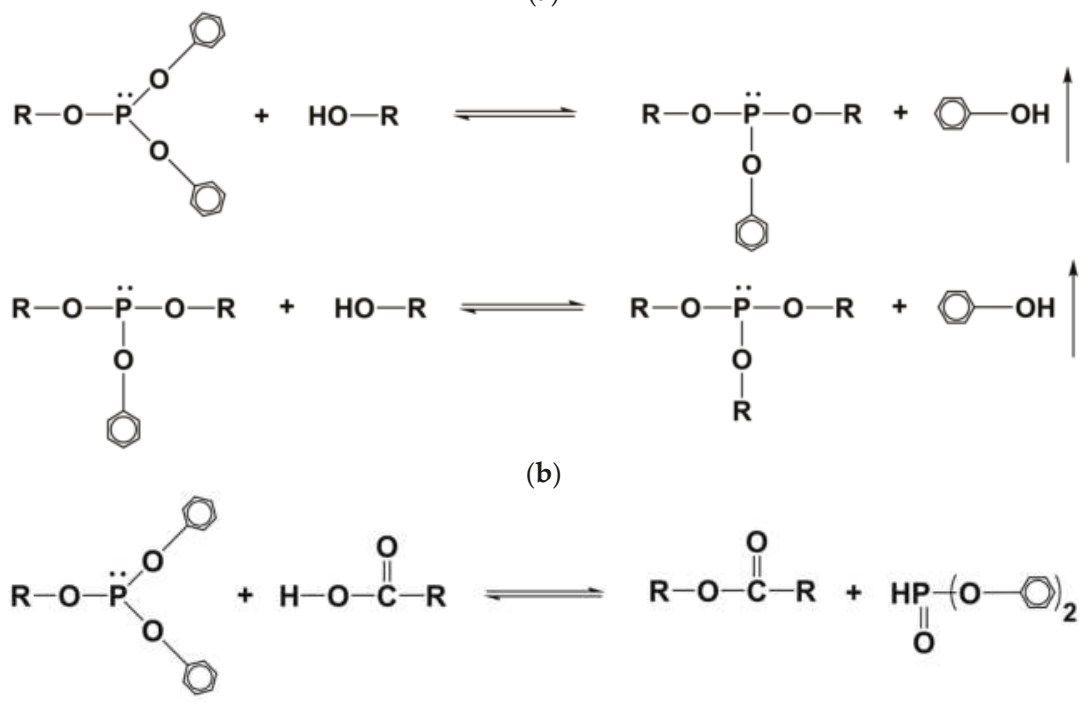

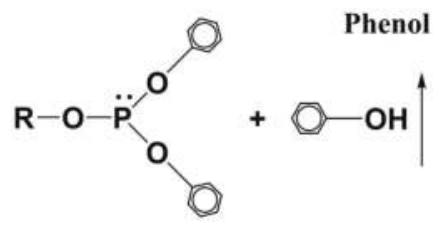

Intermediate alkyl diphenyl phosphite

(c)

Figure 4. Reaction schemes of (a) the initiation of the reaction between triphenyl phosphite (TPP) and polylactic acid (PLA)/poly(butylene succinate-co-adipate) (PBSA); (b) the propagation reaction inducing a possible branching mechanism between the hydroxyl ends of PLA/PBSA polymeric chains; and (c) the propagation reaction inducing a possible chain extension mechanism between the hydroxyl chain ends of PLA/PBSA polymeric chains. Adapted with permission from [27]. Copyright 2013, American Chemical Society.

In the first reaction mechanism, the second step involves a multi-substitution reaction of intermediate alkyl diphenyl phosphite whereby phenoxy groups are replaced with alkyl groups along with the elimination of phenol, as shown in Figure 4b. It is highly likely that this reaction continues until phosphorus serves as a binding point for the occurrence of grafting/branching [33]. In contrast, the second mechanism involves ester linkages from polymers, with phenoxy groups of intermediate product reacting with carboxyl groups of PLA/PBSA (instead of hydroxyl end-groups), leading to a chain extension without $\mathrm{P}$ atoms becoming part of the polymeric chain (Figure 1c).

In all of the above-mentioned reaction schemes, chain extension and/or branching may occur. In our case, compatibilized blends show a marginal increase in molecular weight (Table 3) compared to PLA, indicating that branching is a major reaction pathway. This has been observed in a previous study conducted by Jacues et al. [35], where 2 wt \% TPP was used to melt-blend PET/PBT in a ratio 
of 70:30 [35]. The authors observed a small increase in $M_{n}$, accompanied by branching of both polyesters, as proven by an increase in torque oscillations. Harada et al. [37] observed a similar trend for compatibilized PLA-PBSA blends involving the use of lyscine triisocyanate as a coupling agent, with cross-linking behavior being reported and accompanied by a small increase in the $M_{\mathrm{n}}$ of the PLA blends. Further studies involving multi-detector gel permeation chromatography GPC using viscometry and light scattering might be required to ascertain the exact nature of branching.

\subsection{Crystallization \& Melting Behavior}

Typically, semi-crystalline polymers such as PLA and PBSA can exhibit three kinds of crystallization behaviors - melt crystallization, cold crystallization, and recrystallization-depending on the heating/cooling rate adopted. Melt crystallization refers to the formation of crystals during cooling. Cold crystallization is the ability of amorphous domains to crystallize during heating, while re-crystallization refers to the reorientation of crystals formed during melt/cold crystallization $[6,38]$. In our study, the observation during the first heating pertains to the behavior of injection-molded samples, which were typically subjected to high cooling rates $\left(\sim 200{ }^{\circ} \mathrm{C} / \mathrm{min}\right)$, leading to insufficient time available for crystallization. The second heating cycle erases the prior thermal history of the samples while subjecting them to a low cooling rate in the first cooling cycle $\left(5^{\circ} \mathrm{C} / \mathrm{min}\right)$, and is indicative of the behavior of the nascent material [14]. Hence, the differences observed in the behavior of all samples between both heating cycles in this study, such as (1) the occurrence of cold crystallization only in the first heating cycle; (2) the presence of a single melting peak in the first cycle vs. double melting peaks in the second heating cycle (both corresponding to PLA) in few samples; and (3) an enhanced crystallinity of samples after the second heating cycle; all of which can be attributed to the stark difference in cooling rate.

The presence of $T_{\mathrm{cc}}$ (corresponding to PLA) reported in Tables 4 and 5 during the first heating cycle and its absence in the second heating cycle was because all amorphous molecular domains had crystallized in the first cooling cycle upon use of a slow cooling rate $\left(5^{\circ} \mathrm{C} / \mathrm{min}\right)$. This is in good agreement with the observed increase in the crystallinity of blends from the first heating cycle to the second one, as it indicates that amorphous domains crystallized during the first cooling cycle. Pilla et al. [14] observed similar behavior in the case of PLA/MWCNT (multi-wall carbon nanotubes) composites.

The absence of cold crystallization (corresponding to PBSA) in PBSA samples and blends could be due to several factors. First, PBSA molecules tend to undergo a faster rate of crystallization during cooling, leading to an absence of amorphous domains that could crystallize during reheating [39]. Second, in the case of blend samples, the presence of stiff PLA chains hinders the cold crystallization of PBSA [29], further making its occurrence impossible in blends. With regard to blends, the physical PLA/PBSA blends showed a reduction in $T_{\mathrm{cc}}$ compared to the pure PLA samples, which could be attributed to the possible intermingling of chains of both polymers at the interfaces, resulting in the early onset of crystallization [29]. A further decrease in $T_{\mathrm{cc}}$ was observed for chemically compatibilized blends to $\sim 71^{\circ} \mathrm{C}$, which could be attributed to the enhanced compatibility between PLA and PBSA chains [27].

The reduction in melting temperatures in the compatibilized blend of around $\sim{ }^{\circ} \mathrm{C}$ in both heating cycles was due to a stronger interaction between PLA and PBSA chain segments upon the addition of triphenyl phosphite (TPP), as TPP enhances the mobility of PLA chain segments [27]. This finding is in good agreement with Ojijo et al. [27], who observed a similar lowering in the $T_{\mathrm{m}}$ (to $\sim 152{ }^{\circ} \mathrm{C}$ ) of compatibilized PLA/PBSA blends prepared via use of similar coupling agents.

Furthermore, solid blends (P-S and C-S) exhibited double melting peaks that were due to the melting of PLA crystals with different morphologies [15]. Ojijo et al. [29] had observed that PBSA in molten form has a nucleating effect on the crystallization of PLA, forming crystals of different sizes and morphologies. Hence, the observed double melting peaks was mainly due to the nucleating effect of PBSA. This is due to the inability of simultaneous crystallization of both polymers occurring due to the large difference in their melting temperatures. However, their foamed counterparts (P-1, P-2, 
C-1, and C-2) showed only one melting peak corresponding to a melting of PLA. This indicates that foaming had a strong impact on the reorientation of crystal structures, leading to the formation of highly ordered crystals, even as TPP induced strong compatibilization between PLA and PBSA.

The addition of talc also resulted in the obtainment of double melting peaks in PLA (AT-S, AT-1, and AT-2) and physical blends (PT-S, PT-1, and PT-2), which could be due to the heterogeneous nucleation effect of talc particles resulting in the obtainment of varying crystal sizes, which is in agreement with other literature [40-42]. Interestingly, compatibilized blends showed only one melting peak upon the addition of talc-in stark contrast with the above-mentioned observation. This can be explained by the reinforcing effect of talc, which enhances bulk crystallinity without impacting crystal size, as observed by Tanniru and Misra et al. [43] for $\mathrm{CaCO}_{3}$-reinforced PE composites.

The crystallinity of foamed compatibilized blends was higher compared to the physically blended counterparts, a phenomenon also observed by Yang et al. [44] on PLA-PBSA compatibilized blends, who attributed this to branching sites acting as nucleation points, leading to a higher probability of nucleation compared to the physical blends. This is in good agreement with our molecular weight results, measured by GPC, indicating a possible occurrence of grafting/branching. For both physical and chemically compatibilized blends, foaming resulted in a higher degree of crystallinity. This could also be attributed to the biaxial extensional flow of ScF affecting the orientation of polymer molecules around cell walls due to foaming, leading to strain-induced crystallization, which results in an increase in the final crystallinity, as observed by Ameli et al. [45]. A similar trend was also observed by Zhai et al. [46] in using chemical foaming agents to foam polycaprolactone. The addition of talc led to an increase in crystallinity for most samples, which could be attributed to the nucleating effect of talc.

\subsection{Viscoelastic Behavior}

The storage modulus is a measure of energy storage and recovery exhibited during cyclic deformation, reflecting the elastic moduli of a material. In general, the storage modulus of any given material can be altered via addition of fillers. Generally, an addition of inorganic fillers is known to enhance the storage modulus of PLA $[41,42,47]$. However, the opposite trend was observed in the pure PLA in this study (A-S and AT-S), which could be due to the inability of talc to exhibit a reinforcing effect. In general, the reinforcing effect of talc is more pronounced in a material exhibiting less stiffness, as explained by Tanniru and Misra [43], who have observed a similar effect of fillers on polymeric materials with reduced stiffness. The pure PLA used in this study exhibited a storage modulus of $3050 \mathrm{MPa}$ at $40^{\circ} \mathrm{C}$, which is far higher than the storage modulus of both pure PLA (2450 MPa) and PLA containing $10 \mathrm{wt} \%$ of silane-treated wood fiber (2556 MPa) reported by Pilla et al. [39]. This excessively high storage modulus of pure PLA used in our study might be a contributing factor towards the lack of any reinforcing effect of talc in the talc-filled PLA samples. However, the opposite trend was observed for both PBSA-based and blended samples due to the elastomeric nature and resultant lower stiffness of PBSA, resulting in an improvement in the storage modulus upon the addition of talc. Among solid blends, compatibilized blends showed a lower storage modulus vis-à-vis physical blends, primarily due to the hindrance in chain movement on account of the possible branching that prevented chain realignment/packing, as observed by Khonakdar et al. in crosslinked HDPE (High-density polyethylene) [48]. Similar phenomena was observed by Ibrahim et al. [49] for cross-linked PLA/PCL (poly(e-caprolactone)) blends compared to physical PLA/PCL blends, and was attributed to the creation of voids in the system upon the formation of the crosslinking network. The compatibilized foamed blends showed a higher storage modulus in this study compared to their physically foamed counterparts, which could be attributed to the higher crystallinity (observed in Tables 5 and 7) due to the synergistic effect of TPP and ScF on crystallinity.

With regard to glass transition temperature, the absence of the plateau region in the storage modulus curve was observed for blend compositions, and can be attributed to the extremely low $T_{\mathrm{g}}$ value of PBSA $\left(\sim-40^{\circ} \mathrm{C}\right)$. Similar observations have been made in another study by Ibrahim et al. [49], where no plateau region was observed in the storage modulus curve of PLA/PCL blends on account of 
a low $T_{\mathrm{g}}$ value of PCL $\left(\sim-60^{\circ} \mathrm{C}\right)$. Ojijo et al. [29], in their study on PLA/PBSA blends, also observed similar trends, and attributed the absence of a plateau region to an increased mobility of PBSA chains above its $T_{\mathrm{g}}\left(\sim-40^{\circ} \mathrm{C}\right)$, leading to a lowering of blend stiffness.

Storage modulus was observed to undergo a sudden increase after a glass transition, corresponding to PLA, for all PLA-containing compositions. This increase was analogous to the cold crystallization from the first heating cycle of DSC, which is in accordance with Zhang et al. [32] where cold crystallization was observed for both individual and blend compositions (PLA-PHBV (polyhydroxybutyrate-valerate)-PBS (poly(butylene succinate))) after glass transition. The appearance of $\mathrm{T}_{c c}$ can be explained by the fact that, for both individual and blend samples, the DMA (Dynamic Mechanical Analyzer) tests were undertaken on injection-molded samples that possessed low crystallinity levels due to the use of high cooling rates (as explained in Section 4.2). Such low crystallinity levels indicated a significant presence of amorphous domains available for crystallization during heating in DMA, allowing them to crystallize post-glass transition, along with an associated sudden increase in storage moduli. With regard to blend compositions, the presence of molten PBSA as nucleating agents acted as an additional factor in enhancing the crystallinity and the subsequent jump in storage moduli [29].

The trends observed for the glass transition temperature $\left(T_{\mathrm{g}}\right)$ in storage moduli curves and $\tan \delta$ curves were in good agreement with each other for all samples (Figures 3 and 4). In the tan $\delta$ curve, a peak was observed in the region where, with increases in temperature, the rate of the decrease in storage modulus was higher than that of the loss modulus. Temperatures corresponding to the $\tan \delta$ peak is often considered as $T_{\mathrm{g}}$. Interestingly, $T_{\mathrm{g}}$ was not observed for the PBSA component in all blend samples due to the locking of PBSA chains by hard PLA segments, thus preventing their motion. Additionally, the use of a lower weight fraction of PBSA meant that a higher share of PBSA chains were restricted by PLA chain segments, ensuring that no $T_{\mathrm{g}}$ corresponding to PBSA was observed for blend compositions [29].

Glass transition temperature of blend samples gives us insight into the miscibility of pure polymers constituting the blends. $T_{\mathrm{g}}$ is typically dependent on the polymer composition of blends, and lies between the $T_{\mathrm{g}}$ values of pure constituents for a completely miscible blend [50]. To obtain clarity on the miscibility and effect of TPP on PLA-PBSA blends, a simplified version of the Gordon-Taylor (G-T) equation (Equation (3)) [51] was applied to $T_{\mathrm{g}}$ obtained from $\tan \delta$.

$$
T_{\mathrm{g}}=\frac{W_{1} T_{\mathrm{g} 1}+k W_{2} T_{\mathrm{g} 2}}{W_{1}+k W_{2}}
$$

Here, $T_{\mathrm{g} 1}$ and $T_{\mathrm{g} 2}$ are the glass transition temperatures of pure components PLA and PBSA, respectively, while $W_{1}$ and $W_{2}$ are the wt $\%$ of PLA and PBSA, respectively, and $k$ is a curve-fitting factor representing the miscibility of the system, with $k=1$ indicating the complete miscibility of the polymers and the lower/higher values of $k$ indicating poor miscibility. Figure 5 depicts $T_{\mathrm{g}}$ of different blend compositions. Observed $T_{\mathrm{g}}$ values for A-S, P-S, and B-S $\left(\sim 75.1, \sim 72\right.$, and $\left.\sim-27.4^{\circ} \mathrm{C}\right)$ and A-S, C-S, and B-S $\left(\sim 75.1, \sim 64\right.$, and $\left.\sim-27.4{ }^{\circ} \mathrm{C}\right)$ were plotted as the $T_{\mathrm{g}}$ of the talc-filled and ScF-foamed blends, all of which were found to overlap (Table 8). These observed values were closer to the G-T curve for $k=0.08$ and $k=0.25$, where the curve-fitting parameter $k$ showed a value of 0.08 for the physical blends, indicating the poor miscibility of PLA and PBSA, as they are thermodynamically immiscible [52]. However, an addition of $2 \mathrm{wt} \%$ TPP shifted the $T_{\mathrm{g}}$ of PLA-PBSA blends to around $64{ }^{\circ} \mathrm{C}$, with the $k$ value of 0.25 used to curve fit the G-T equation; this higher value of $k$ indicates the possibility of enhanced compatibilization. 


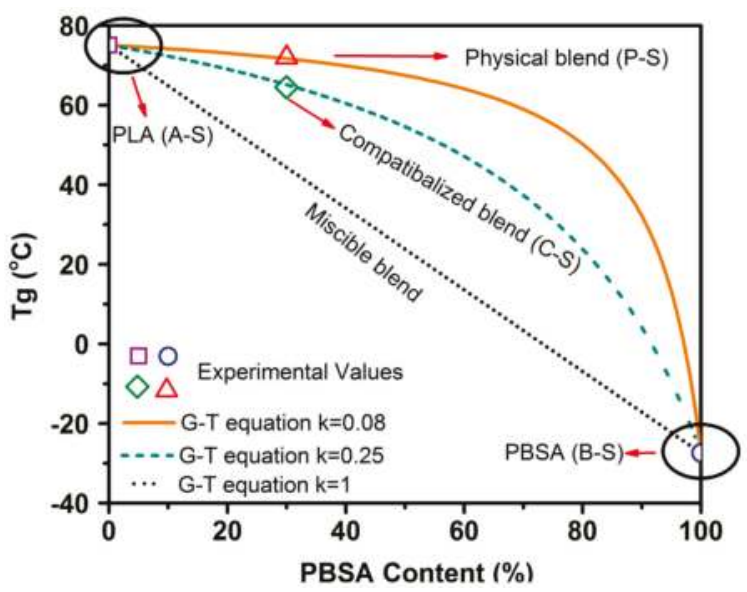

Figure 5. Comparison of the experimental and theoretical $T_{\mathrm{g}}$ values of PLA-PBSA blends.

\section{Conclusions}

Compatibilized blends of PLA and PBSA were successfully processed using TPP via reactive extrusion and foamed via ScF-assisted injection molding technology. The compatibilization was verified via an improvement in $M_{\mathrm{n}}$ using GPC, a shift in $T_{\mathrm{g}}$ using DSC, and an improved miscibility as shown by the G-T equation. Thermal properties of solid and foamed samples, studied using DSC, revealed that the addition of talc/compatibilizer and the use of ScF foaming had a significant impact on crystallinity, melt, cold crystallization, and glass transition temperatures. Compatibilized ScF-foamed blends showed an improvement in crystallinity by $\sim 10 \%$ over their physical blend unfoamed counterparts. The viscoelastic properties of the samples revealed further evidence of compatibilization, as verified by the G-T equation. Furthermore, compatibilized foamed blends showed superior storage moduli compared to their physically foamed counterparts due to the synergistic effect of TPP and ScF on crystallinity.

Acknowledgments: The authors are grateful to Kimberly Ivey for her assistance in conducting TGA, DSC, and DMA tests. Sai Aditya Pradeep would like to acknowledge the support of the Sonoco Fellowship.

Author Contributions: Sai Aditya Pradeep carried out all experiments and analyses. Srikanth Pilla conceived the experiments and directed the research. Hrishikesh Kharbas and Lih-Sheng Turng assisted with ScF experiments. Abraham Avalos and Joseph G. Lawrence assisted with extrusion experiments. All authors participated in discussions of the research, and Sai Aditya Pradeep and Srikanth Pilla wrote the manuscript.

Conflicts of Interest: The authors declare no conflict of financial interest. The funding sponsors had no role in the design of the study; in the collection, analyses or interpretation of data; or in the writing of the manuscript.

\section{References}

1. Landrock, A.H. Handbook of Plastic Foams: Types, Properties, Manufacture and Applications; Elsevier: Amsterdam, The Netherlands, 1995.

2. Urayama, H.; Kanamori, T.; Kimura, Y. Properties and biodegradability of polymer blends of poly(L-lactide)s with different optical purity of the lactate units. Macromol. Mater. Eng. 2002, 287, 116-121. [CrossRef]

3. Urayama, H.; Ma, C.; Kimura, Y. Mechanical and thermal properties of poly(L-lactide) incorporating various inorganic fillers with particle and whisker shapes. Macromol. Mater. Eng. 2003, 288, 562-568. [CrossRef]

4. Suyatma, N.E.; Copinet, A.; Tighzert, L.; Coma, V. Mechanical and barrier properties of biodegradable films made from chitosan and poly (lactic acid) blends. J. Polym. Environ. 2004, 12, 1-6. [CrossRef]

5. Auras, R.A.; Harte, B.; Selke, S.; Hernandez, R. Mechanical, physical, and barrier properties of poly(lactide) films. J. Plast. Film Sheeting 2003, 19, 123-135. [CrossRef] 
6. Pilla, S.; Gong, S.; O’Neill, E.; Yang, L.; Rowell, R.M. Polylactide-recycled wood fiber composites. J. Appl. Polym. Sci. 2009, 111, 37-47. [CrossRef]

7. Huda, M.S.; Drzal, L.T.; Misra, M.; Mohanty, A.K.; Williams, K.; Mielewski, D.F. A study on biocomposites from recycled newspaper fiber and poly(lactic acid). Ind. Eng. Chem. Res. 2005, 44, 5593-5601. [CrossRef]

8. Grijpma, D.W.; van Hofslot, R.D.A.; Supèr, H.; Nijenhuis, A.J.; Pennings, A.J. Rubber toughening of poly(lactide) by blending and block copolymerization. Polym. Eng. Sci. 1994, 34, 1674-1684. [CrossRef]

9. Odent, J.; Leclère, P. Toughening of polylactide by tailoring phase-morphology with P[CL-co-LA] random copolyesters as biodegradable impact modifiers. Eur. Polym. J. 2013, 49, 914-922. [CrossRef]

10. Odent, J.; Raquez, J.-M.; Dubois, P. Highly toughened polylactide-based materials through melt-blending techniques. In Biodegradable Polyesters; Wiley-VCH Verlag GmbH \& Co., KGaA: Weinheim, Germany, 2015; pp. 235-274.

11. Zeng, J.-B.; Li, K.-A.; Du, A.-K. Compatibilization strategies in poly(lactic acid)-based blends. RSC Adv. 2015, 5, 32546-32565. [CrossRef]

12. Kfoury, G.; Raquez, J.-M.; Hassouna, F.; Odent, J.; Toniazzo, V.; Ruch, D.; Dubois, P. Recent advances in high performance poly(lactide): From "green" plasticization to super-tough materials via (reactive) compounding. Front. Chem. 2013, 1, 32. [CrossRef] [PubMed]

13. Nagarajan, V.; Mohanty, A.K.; Misra, M. Perspective on polylactic acid (PLA) based sustainable materials for durable applications: Focus on toughness and heat resistance. ACS Sustain. Chem. Eng. 2016, 4, 2899-2916. [CrossRef]

14. Pilla, S.; Kramschuster, A.; Gong, S.; Chandra, A.; Turng, L.-S. Solid and microcellular polylactide-carbon nanotube nanocomposites. Int. Polym. Process. 2007, 22, 418-428. [CrossRef]

15. Pilla, S.; Kramschuster, A.; Yang, L.; Lee, J.; Gong, S.; Turng, L.-S. Microcellular injection-molding of polylactide with chain-extender. Mater. Sci. Eng. C 2009, 29, 1258-1265. [CrossRef]

16. Kwag, C.; Manke, C.W.; Gulari, E. Rheology of molten polystyrene with dissolved supercritical and near-critical gases. J. Polym. Sci. Part B 1999, 37, 2771-2781. [CrossRef]

17. Kwag, C.; Manke, C.W.; Gulari, E. Effects of dissolved gas on viscoelastic scaling and glass transition temperature of polystyrene melts. Ind. Eng. Chem. Res. 2001, 40, 3048-3052. [CrossRef]

18. Gong, S.; Yuan, M.; Chandra, A.; Kharbas, H.; Osorio, A.; Turng, L.S. Microcellular injection molding. Int. Polym. Process. 2005, 20, 202-214. [CrossRef]

19. Lee, J.; Turng, L.-S. Improving surface quality of microcellular injection molded parts through mold surface temperature manipulation with thin film insulation. Polym. Eng. Sci. 2010, 50, 1281-1289. [CrossRef]

20. Chandra, A.; Gong, S.; Yuan, M.; Turng, L.-S.; Gramann, P.; Cordes, H. Microstructure and crystallography in microcellular injection-molded polyamide-6 nanocomposite and neat resin. Polym. Eng. Sci. 2005, 45, 52-61. [CrossRef]

21. Yuan, M.; Turng, L.-S.; Gong, S.; Caulfield, D.; Hunt, C.; Spindler, R. Study of injection molded microcellular polyamide-6 nanocomposites. Polym. Eng. Sci. 2004, 44, 673-686. [CrossRef]

22. Wu, D.; Lin, D.; Zhang, J.; Zhou, W.; Zhang, M.; Zhang, Y.; Wang, D.; Lin, B. Selective localization of nanofillers: Effect on morphology and crystallization of PLA/PCL blends. Macromol. Chem. Phys. 2011, 212, 613-626. [CrossRef]

23. Abdelwahab, M.A.; Flynn, A.; Chiou, B.-S.; Imam, S.; Orts, W.; Chiellini, E. Thermal, mechanical and morphological characterization of plasticized PLA-PHB blends. Polym. Degrad. Stab. 2012, 97, 1822-1828. [CrossRef]

24. Zhao, H.; Cui, Z.; Sun, X.; Turng, L.-S.; Peng, X. Morphology and properties of injection molded solid and microcellular polylactic acid/polyhydroxybutyrate-valerate (PLA/PHBV) blends. Ind. Eng. Chem. Res. 2013, 52, 2569-2581. [CrossRef]

25. Yokohara, T.; Yamaguchi, M. Structure and properties for biomass-based polyester blends of PLA and PBS. Eur. Polym. J. 2008, 44, 677-685. [CrossRef]

26. Javadi, A.; Kramschuster, A.J.; Pilla, S.; Lee, J.; Gong, S.; Turng, L.-S. Processing and characterization of microcellular PHBV/PBAT blends. Polym. Eng. Sci. 2010, 50, 1440-1448. [CrossRef]

27. Ojijo, V.; Ray, S.S.; Sadiku, R. Toughening of biodegradable polylactide/poly(butylene succinate-co-adipate) blends via in situ reactive compatibilization. ACS Appl. Mater. Interfaces 2013, 5, 4266-4276. [CrossRef] [PubMed] 
28. Zhao, H.; Cui, Z.; Wang, X.; Turng, L.-S.; Peng, X. Processing and characterization of solid and microcellular poly(lactic acid)/polyhydroxybutyrate-valerate (PLA/PHBV) blends and PLA/PHBV/clay nanocomposites. Compos. Part B 2013, 51, 79-91. [CrossRef]

29. Ojijo, V.; Sinha Ray, S.; Sadiku, R. Role of specific interfacial area in controlling properties of immiscible blends of biodegradable polylactide and poly[(butylene succinate)-co-adipate]. ACS Appl. Mater. Interfaces 2012, 4, 6690-6701. [CrossRef] [PubMed]

30. Pilla, S.; Kramschuster, A.; Lee, J.; Clemons, C.; Gong, S.; Turng, L.-S. Microcellular processing of polylactide-hyperbranched polyester-nanoclay composites. J. Mater. Sci. 2010, 45, 2732-2746. [CrossRef]

31. Bureepukdee, C.; Suttiruengwong, S.; Seadan, M. A study on reactive blending of (poly lactic acid) and poly (butylene succinate co adipate). IOP Conf. Ser. Mater. Sci. Eng. 2015, 87, 12070. [CrossRef]

32. Zhang, K.; Mohanty, A.K.; Misra, M. Fully biodegradable and biorenewable ternary blends from polylactide, poly(3-hydroxybutyrate-co-hydroxyvalerate) and poly(butylene succinate) with balanced properties. ACS Appl. Mater. Interfaces 2012, 4, 3091-3101. [CrossRef] [PubMed]

33. Koning, C.; van Duin, M.; Pagnoulle, C.; Jerome, R. Strategies for compatibilization of polymer blends. Prog. Polym. Sci. 1998, 23, 707-757. [CrossRef]

34. Aycock, D.F.; Ting, S.P. Polyphenylene Ether-Polyamide Blends. U.S. Patent 460,074,1 A, 15 July 1986.

35. Jacques, B.; Devaux, J.; Legras, R.; Nield, E. Reactions induced by triphenyl phosphite addition during melt mixing of PET/PBT blends: Chromatographic evidence of a molecular weight increase due to the creation of bonds of two different natures. Polymer 1997, 38, 5367-5377. [CrossRef]

36. Aharoni, S.M.; Forbes, C.E.; Hammond, W.B.; Hindenlang, D.M.; Mares, F.; O’Brien, K.; Sedgwick, R.D. High-temperature reactions of hydroxyl and carboxyl PET chain end groups in the presence of aromatic phosphite. J. Polym. Sci. Part A 1986, 24, 1281-1296. [CrossRef]

37. Harada, M.; Ohya, T.; Iida, K.; Hayashi, H.; Hirano, K.; Fukuda, H. Increased impact strength of biodegradable poly(lactic acid)/poly(butylene succinate) blend composites by using isocyanate as a reactive processing agent. J. Appl. Polym. Sci. 2007, 106, 1813-1820. [CrossRef]

38. Lee, Y.; Porter, R.S.; Lin, J.S. On the double-melting behavior of poly(ether ether ketone). Macromolecules 1989, 22, 1756-1760. [CrossRef]

39. Pilla, S.; Gong, S.; O’Neill, E.; Rowell, R.M.; Krzysik, A.M. Polylactide-pine wood flour composites. Polym. Eng. Sci. 2008, 48, 578-587. [CrossRef]

40. Jain, S.; Misra, M.; Mohanty, A.K.; Ghosh, A.K. Thermal, mechanical and rheological behavior of poly(lactic acid)/talc composites. J. Polym. Environ. 2012, 20, 1027-1037. [CrossRef]

41. Fowlks, A.C.; Narayan, R. The effect of maleated polylactic acid (PLA) as an interfacial modifier in PLA-talc composites. J. Appl. Polym. Sci. 2010, 118, 2810-2820. [CrossRef]

42. Cipriano, T.F.; da Silva, A.L.N.; da Fonseca Thomé da Silva, A.H.M.; de Sousa, A.M.F.; da Silva, G.M.; Rocha, M.G. Thermal, rheological and morphological properties of poly (lactic acid) (PLA) and talc composites. Polímeros 2014, 24, 276-282.

43. Tanniru, M.; Misra, R.D.K. On enhanced impact strength of calcium carbonate-reinforced high-density polyethylene composites. Mater. Sci. Eng. A 2005, 405, 178-193. [CrossRef]

44. Yang, X.; Xu, H.; Odelius, K.; Hakkarainen, M. Poly(lactide)-g-poly(butylene succinate-co-adipate) with high crystallization capacity and migration resistance. Materials 2016, 9, 313. [CrossRef]

45. Ameli, A.; Jahani, D.; Nofar, M.; Jung, P.U.; Park, C.B. Development of high void fraction polylactide composite foams using injection molding: Mechanical and thermal insulation properties. Compos. Sci. Technol. 2014, 90, 88-95. [CrossRef]

46. Zhai, W.; Kuboki, T.; Wang, L.; Park, C.B.; Lee, E.K.; Naguib, H.E. Cell structure evolution and the crystallization behavior of polypropylene/clay nanocomposites foams blown in continuous extrusion. Ind. Eng. Chem. Res. 2010, 49, 9834-9845. [CrossRef]

47. Liu, X.; Wang, T.; Chow, L.C.; Yang, M.; Mitchell, J.W. Effects of inorganic fillers on the thermal and mechanical properties of poly(lactic acid). Int. J. Polym. Sci. 2014, 2014, 827028. [CrossRef] [PubMed]

48. Khonakdar, H.A.; Morshedian, J.; Wagenknecht, U.; Jafari, S.H. An investigation of chemical crosslinking effect on properties of high-density polyethylene. Polymer 2003, 44, 4301-4309. [CrossRef]

49. Ibrahim, A.N.; Wahit, M.U.; Yussuf, A.A. Effect of fiber reinforcement on mechanical and thermal properties of poly( $\varepsilon$-caprolactone)/poly(lactic acid) blend composites. Fibers Polym. 2014, 15, 574-582. [CrossRef] 
50. Krutphun, P.; Supaphol, P. Thermal and crystallization characteristics of poly(trimethylene terephthalate)/ poly(ethylene naphthalate) blends. Eur. Polym. J. 2005, 41, 1561-1568. [CrossRef]

51. Gordon, M.; Taylor, J.S. Ideal copolymers and the second-order transitions of synthetic rubbers. I. non-crystalline copolymers. J. Appl. Chem. 2007, 2, 493-500.

52. Eslami, H.; Kamal, M.R. Effect of a chain extender on the rheological and mechanical properties of biodegradable poly(lactic acid)/poly[(butylene succinate)-co-adipate] blends. J. Appl. Polym. Sci. 2013, 129, 2418-2428. [CrossRef]

(C) 2017 by the authors. Licensee MDPI, Basel, Switzerland. This article is an open access article distributed under the terms and conditions of the Creative Commons Attribution (CC BY) license (http://creativecommons.org/licenses/by/4.0/). 
Article

\title{
All-Inorganic Intumescent Nanocoating Containing Montmorillonite Nanoplatelets in Ammonium Polyphosphate Matrix Capable of Preventing Cotton Ignition
}

\author{
Jenny Alongi ${ }^{1, *}$ and Federico Carosio ${ }^{2}$ \\ 1 Dipartimento di Chimica, Università degli Studi di Milano, Via Golgi 19, 20133 Milano, Italy \\ 2 Dipartimento di Scienza Applicata e Tecnologia, Politecnico di Torino, Alessandria Site, \\ Viale Teresa Michel 5, 15121 Alessandria, Italy; federico.carosio@polito.it \\ * Correspondence: jenny.alongi@unimi.it; Tel.: +39-02-50314108
}

Academic Editor: Alexander Böker

Received: 29 October 2016; Accepted: 8 December 2016; Published: 10 December 2016

\begin{abstract}
In the present manuscript a new concept of completely inorganic intumescent flame retardant nanocoating comprised of sodium montmorillonite nanoplatelets embedded in an ammonium polyphosphate matrix has been investigated using cotton as model substrate. The coating, deposited by multistep adsorption from diluted water-based suspensions/solutions, homogenously cover each cotton fibers with average thicknesses below $50 \mathrm{~nm}$ and add-on up to $5 \%$ in weight. Combustion characterization evidences the interesting properties: indeed, the so-treated fabrics reached self-extinguishing during horizontal flame spread tests. Furthermore, when the coating add-on reaches $5 \%$, no ignition has been observed during cone calorimetry tests under $35 \mathrm{~kW} / \mathrm{m}^{2}$ heat flux. Residue analyses pointed out the formation of an expanded all-inorganic coating capable of greatly improving char formation by exerting barrier function towards volatile release and heat transfer.
\end{abstract}

Keywords: cotton; flame retardancy; combustion; intumescence; sodium cloisite; APP

\section{Introduction}

In recent years, the demand for new and sustainable materials has grown and spread in several research fields with the aim of replacing old and inefficient materials concepts with innovative and efficient solutions. In particular, the design of fire safe materials is an area of great concern since the safety and efficiency of conventionally adopted chemistry have been questioned due to perceived human and environment hazards (e.g., halogen-based compounds have been found in the food chain, dangerously ending in the bodies of animals and humans) [1-3]. Initial countermeasures to this problem have led to restrain the use of some flame-retardants (FRs) and to start a campaign aiming to evaluate the benefit to danger ratio for the remaining chemicals [4]. In this context, finding non-toxic and high-performing fire retardant solutions is of great industrial and scientific interest.

Nanotechnology represents a possible tool for achieving such goal. Indeed, in the field of materials science, nanomaterials have demonstrated to possess superior properties due to the achieved nanostructures. One clear example of nanostructured materials is represented by polymer-layered nanocomposites; this class of materials has demonstrated remarkable improvements in mechanical strength, oxygen barrier properties and flammability with respect to neat polymers [5]. For instance, the use of lamellar shaped nanoparticles allowed for obtaining peculiar gas barrier and flame retardant properties; indeed, in the former case, when homogeneously dispersed, nanoparticles would create a tortuous path capable of slowing down gas molecule diffusions through polymer matrix while in the 
latter they would allow for the formation of an inorganic barrier by cumulating on top of a burning polymer and reducing the combustion kinetics [6]. Such results were achieved with low amounts of inorganic filler (usually below $5 \mathrm{wt} \%$ ) with respect of conventional polymer micro-composites and were tightly bonded for obtaining a nanostructure in which almost each nanoparticle is isolated from the other ones. However, the nanostructuring was not easy to achieve as a lot of efforts during processing and time-consuming modifications of the nanoparticles had to be made in order to homogeneously disperse the nanoparticles within polymer matrix [7].

Recent literature clearly demonstrates that it is possible to overcome such problems, specifically in the field of gas barrier and flame retardancy, by changing the approach towards nanostructuring $[8,9]$. In this concept, nanoparticles are removed from the bulk in order to be deposited or assemble on the surface, thus resulting in a nanoscale or nanostructured coating capable of greatly improving the performances of the coated polymer. For instance, a PLA (poly(lactic acid))-film coated with efficiently organized nanoplatelets (ideally aligned parallel to the surface and perpendicularly to the gas flux) may reach a oxygen permeability several orders of magnitude lower than that of neat polymer with a substantial improvement of barrier properties with respect to bulk nanocomposites [10,11]. On the other hand, the surface approach for flame retardancy has been demonstrated as a facile and straightforward path to impressive results. This path has been aided by different surface modification techniques based on the deposition from aqueous based suspensions of nanoparticles such as the Layer-by-Layer assembly (LbL) or the simpler nanoparticle adsorption $[9,12,13]$. In both approaches, the substrate is exposed to a nanoparticle suspension in order to have the adsorption on the surface. This can be performed one time such as in nanoparticle adsorption or multiple times as in LbL assembly. By relying on the electrostatic attractions occurring between nanoparticles and polyelectrolytes in water, the latter approach deposits differently charged species at each adsorption step in order to growth a coating by stacking negatively and positively charged layers [14,15]. Due to the availability of different nanoparticles and polyelectrolytes, many FR actions have been targeted through years. The first and most simple coatings were assembled for obtaining a completely inorganic layer made of nanoparticles; to this aim, sodium montmorillonite or silica nanoparticles have been used on fabrics (cotton and polyester) or thick bulk polymers (polycarbonate (PC), polyamide (PA) and polyester (PET) ) [16-19]. The collected results showed the efficiency of this approach; indeed, the coatings were able to suppress the melt-dripping of PC and PET and to considerably slow down the combustion kinetics of these substrates, often allowing for an unexpected increase in the time to ignition (TTI) [17]. The latter result can be considered unexpected as it is in evidenced contrast with the behavior of nanoparticles observed in bulk nanocomposites where their inclusion in a polymer matrix almost systematically reduced TTI [20].

Through years of research, more complicated coating compositions have been experimented and proven successful [21-23]. Of particular interest are the coatings based on the concept of intumescence; their main FR action is due to the formation of an expanded charred structure on the surface of the burning polymer with consequent reduction of heat transmitted from the flame and combustible volatile release $[24,25]$. This mimics the FR of classical intumescent coatings that are macroscopically bigger reaching several hundreds of microns in thickness [26]. Intumescence in nanostructured coatings can be achieved by ensuring the presence of an acid source, a carbon source and a blowing agent within the coating structure; a simple example is represented by chitosan $(\mathrm{CH})$ /ammonium polyphosphate (APP) coatings where $\mathrm{CH}$ acts as carbon source and APP provides phosphoric acid and ammonia as blowing agent [27]. Intumescent coatings normally provided better results than nanoparticle containing ones; as an example, cotton can achieve self-extinguishment behavior during flammability tests only with the deposition of a polyallylamine/polyphosphate or starch/polyphosphate coating [28,29]. The FR action of this kind of coatings has been improved by the inclusion of nanoparticles, thus resulting in a hybrid organic expanded structure reinforced by the inorganic particles. However, if one flaw has to be pointed out, the presence of the organic expanded part represents a weakness of the 
protective coating as from one hand it can be easily oxidized and from the other hand it possess weak mechanical properties making it prone to collapsing as a consequence of convective motes.

In the present paper we are targeting this flaw by trying to remove the organic part while maintaining the intumescent features of the coating. To this aim, we aim to the deposition of a coating containing sodium montmorillonite (MTM) nanoparticles embedded within an ammonium polyphosphate continuous matrix in order to produce an inorganic expandable structure. The coating concept is derived from the practical observation of what occurs when a mixture of the MTM and APP powders is exposed to a radiant heat flux typical of developing fires. Indeed, the increased temperature triggers the APP dissociation with the release of ammonia and the production of polyphosphoric acid that reacts with MTM nanoplatelets forming of a silicoalluminophosphate expanded structure (see Figure S1 in Supplementary Materials). This and other possible interaction reactions have been already reported in the literature for APP/MTM containing polymers and have been ascribed as one of the reasons for the improved flame retardancy achieved by the combination of the two components with respect to the single constituents [30,31]. Here, we use an easy multi-step adsorption process for the deposition of APP/MTM coatings in order to improve cotton FR properties. Cotton has been selected as model substrate for the evaluation of the coating performances because of its hydrophilic nature and well known degradation process. The morphology of treated and untreated fabrics has been investigated by electron microscopies, the changes in thermal stability have been evaluated by thermogravimetric analyses (in inert and oxidative atmospheres) and the achieved FR characteristics have been assessed by horizontal flame spread tests and cone calorimetry. Finally, the residues collected at the end of the FR tests have been analyzed and a mechanism for the observed FR behavior has been proposed.

\section{Materials and Methods}

\subsection{Materials}

Cotton with an area density of $100 \mathrm{~g} / \mathrm{m}^{2}$ was purchased from Fratelli Ballesio S.r.l. (Torino, Italy). Prior to deposition, cotton fabrics were washed with water and Marseille soap, ethanol and then diethyl ether. After the washing steps, fabrics were dried in an oven at $70{ }^{\circ} \mathrm{C}$ for $1 \mathrm{~h}$.

The sodium montmorillonite was purchased from Southern Clays Products Inc. (Gonzales, TX, USA) and employed in $1 \mathrm{wt} \%$ water suspension. The suspension was kept under magnetic stirring for $24 \mathrm{~h}$ and then centrifuged at $4400 \mathrm{rpm}$ for $5 \mathrm{~min}$ in order to remove aggregates, resulting in a final concentration of $0.7 \mathrm{wt} \%$.

Ammonium polyphosphate (PHOS-CHEK ${ }^{\circledR}$ P30, purchased from ICL Performance Products Inc., Milano, Italy) was used for preparing $1 \mathrm{wt} \%$ water solution that was kept under magnetic stirring for $24 \mathrm{~h}$ prior to use. The $18.2 \mathrm{M} \Omega$ deionized water supplied by a Q20 Millipore system (Milano, Italy) was employed for both APP solutions and MTM suspensions.

\subsection{Coating Deposition}

Cotton fabrics were alternately dipped in the APP solution and then in MTM suspension. In between each adsorption step, the fabrics were squeezed using a Padder model FL300 produced by Gavazzi S.r.l (Bergamo, Italy) and dried in a convection oven at $80^{\circ} \mathrm{C}$ for $30 \mathrm{~min}$, mimicking an impregnation pad-dry industrial treatment. The dipping time was set at $5 \mathrm{~min}$ for the first couple of adsorption steps; subsequent steps were achieved after $1 \mathrm{~min}$. The alternate dipping/padding/drying cycle was repeated 2 or 4 times in order to achieve final coating add-ons of $2.5 \%$ and $5 \%$, respectively. In the following, coated samples are coded as \% add-on APP/MTM.

\subsection{Characterization}

Scanning Electron Microscopy: the change in surface morphology of treated cotton with respect to untreated one was evaluated using a Field-Emission Scanning Electron Microscopy (FE-SEM) on a 
ZEISS, FEG model MERLIN. A LEO-1450VP Scanning Electron Microscope (Carl Zeiss Microscopy $\mathrm{GmbH}$, Jena, Germany) equipped with an X-ray probe (INCA Energy Oxford, Oxfordshire, UK, Cu-K $\alpha$ X-ray source, $k=1.540562 \AA$ ) was used to perform elemental analysis (Energy Dispersive Spectroscopy, EDS). In both cases untreated and treated cotton fabrics were cut $\left(10 \times 10 \mathrm{~mm}^{2}\right)$, fixed to conductive adhesive tapes and either chromium- (FE-SEM) or gold- (SEM) metallized prior to imaging.

X-ray diffraction: X-ray diffraction spectra (XRD) were collected on $30 \times 30 \times 0.5 \mathrm{~mm}^{3}$ samples with a Philips X'Pert-MPD diffractometer (PANalytical, Eindhoven, The Netherlands, Cu-Ka radiation, $k=1.540562 \AA$ 注 step size: 0.02 ; step time: $2 \mathrm{~s}$ ).

Thermal stability: A TAQ500 thermogravimetric balance (TA-Instruments, Milano, Italy) was used for thermogravimetric analyses. The tests were performed from 50 to $800{ }^{\circ} \mathrm{C}$ (heating rate of $\left.10{ }^{\circ} \mathrm{C} / \mathrm{min}\right)$ in both nitrogen and air $(60 \mathrm{~mL} / \mathrm{min})$ on $10 \mathrm{mg}$ samples placed in open alumina pans. The following parameters were assessed: $T_{\text {onset } 5 \%}$ (temperature at which a $5 \mathrm{wt} \%$ weight loss is registered), $T_{\max }$ (temperature at which the maximum weight loss is registered), residue at $T_{\max }$ and $800{ }^{\circ} \mathrm{C}$. The experimental error was $0.5 \%$ on the weight and $1{ }^{\circ} \mathrm{C}$ on the temperature. Thermogravimetric (TG) and derivative (dTG) curves were reported.

Horizontal flame spread tests: The reaction to the flame application of the prepared samples was evaluated in horizontal configuration. During the test the sample $\left(100 \times 50 \mathrm{~mm}^{2}\right)$ was placed in a metallic frame and tilted $45^{\circ}$ with respect to its longer axis, then a $20 \mathrm{~mm}$ blue methane flame is applied to its short side for $3 \mathrm{~s}$ in order to ignite it. Parameters such as burning time, afterglow times and final residue were registered during the test. At least three tests were performed for each formulation.

Cone calorimetry: An oxygen consumption cone calorimeter (Fire Testing Technology, FTT) was employed to investigate the combustion behavior of square samples $\left(100 \times 100 \mathrm{~mm}^{2}\right)$ under $35 \mathrm{~kW} / \mathrm{m}^{2}$ in horizontal configuration. Tests were performed following the ISO 5660 standard implementing the optimized procedure for textiles described elsewhere [32]. The following parameters were registered: Time To Ignition (TTI, (s)), peak of Heat Release Rate (pkHRR, $\left.\left(\mathrm{kW} / \mathrm{m}^{2}\right)\right)$, Total Heat Release (THR, $\left.\left(\mathrm{MJ} / \mathrm{m}^{2}\right)\right)$ and final residue. At least three tests were performed and standard deviation $(\sigma)$ was calculated as experimental error; for non-igniting samples the test was repeated five times.

Fourier Transformed Infrared Spectroscopy in Attenuated Total Reflectance (FT-IR ATR): Spectra were collected at room temperature (range $4000-700 \mathrm{~cm}^{-1}, 16$ scans and $4 \mathrm{~cm}^{-1}$ resolution) using a Frontier FT-IR/FIR spectroscopy (Perkin Elmer, Milano, Italy) in ATR configuration, equipped with a diamond crystal (depth of penetration $1.66 \mu \mathrm{m}$, as stated by the producer).

Raman spectroscopy: Analyses were performed on an InVia Raman Microscope (argon laser source $514 \mathrm{~nm} / 50 \mathrm{~mW}$, Renishaw S.p.A., Torino, Italy) coupled with a Leica DM 2500 optical microscope (Leica Microsystems S.r.l., Milano, Italy).

\section{Results}

\subsection{Coating Morphology on Cotton}

Coating morphology on cotton fibers has been carefully investigated by FE-SEM observation combined with XRD diffraction. Figure 1 reports micrographs of uncoated and APP/MTM-coated cotton and XRD spectra performed on neat MTM powder and 5\% APP/MTM sample.

Neat cotton shows the typical morphology of a natural fiber with a rough and irregular surface. When cotton is coated by APP/MTM, no immediate change in morphology can be detected. Indeed, by a direct comparison of the uncoated and coated fibers, it is really difficult to establish whether a coating has been deposited.

The presence of the coating has been revealed by investigating sites where, as a consequence of deformations, the coating is partially detached from cotton surface. 

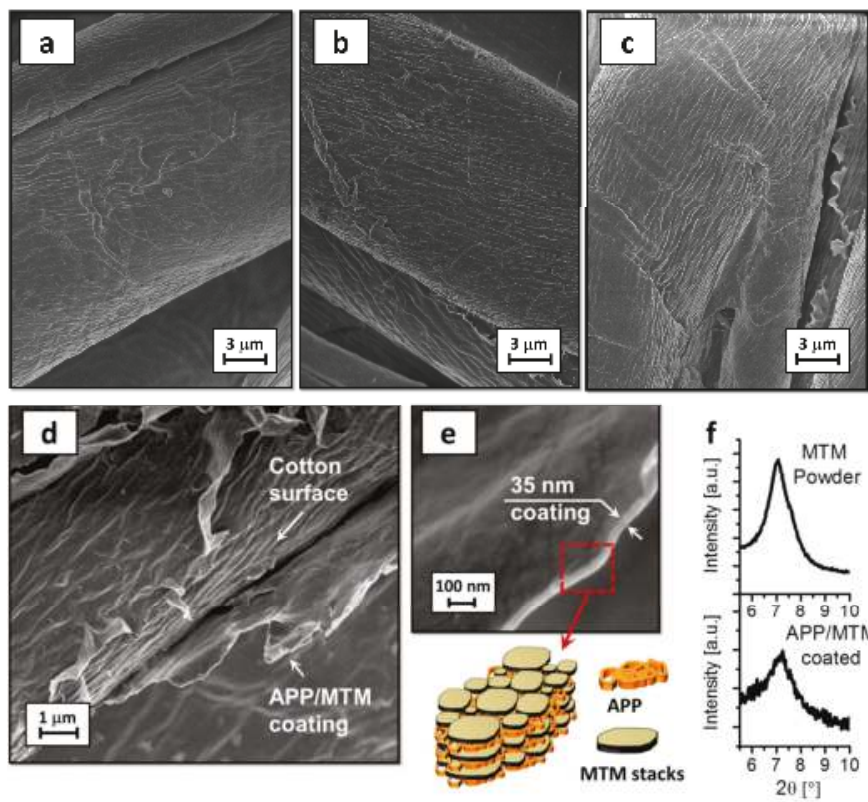

Figure 1. FE-SEM (Field-Emission Scanning Electron Microscopy) micrographs of: neat cotton (a); $2.5 \%$ APP /MTM sample (b); $5 \%$ APP/MTM sample (c); details of 5\% APP/MTM (d,e); and XRD spectra of neat MTM and 5\% APP/MTM fabric (f).

As reported in Figure 1, high magnification micrographs point out the presence of a very thin coating that averages $35 \mathrm{~nm}$ for $5 \%$ APP/MTM samples. Similar investigations are possible on $2.5 \%$ $\mathrm{APP} / \mathrm{MTM}$ and result in thinner and less homogenous coatings, which thickness is more difficult to evaluate (see Figure S2 in Supplementary Materials).

The weight gain after the deposition was found to be around 2.5 and $5 \mathrm{wt} \%$; furthermore, the deposited nanocoating had no impact on fabric hand and color. From the micrographs collected in Figure 1, different information can be gathered: the coating is really thin and when deposited on cotton fibers is capable of literally reproducing the surface irregularities of the original fibers and MTM nanoplatelets are adsorbed on the surface with a strong in-plane orientation.

The last statement is also confirmed by XRD measurements. As reported in the interpherogram, neat MTM shows the characteristic peak at $7.0^{\circ}$ related of the basal spacing between each MTM nanoplatelet, which is consistent with the literature [33,34]. APP/MTM coated fabrics displayed a diffraction peak in the same region indicating that MTM is adsorbed as stacks consisting of several nanoplatelets laying parallel on the surface and are held together by APP in a "brick and mortar-like structure", as schematized in Figure 1 [35].

EDS analyses performed on so-coated samples revealed the presence of elements characteristic of APP (phosphorous) and MTM (silicon), further confirming the presence of both reagents within the coating (see Figure S3 in Supplementary Materials). By evaluating the different Si and P percentages, it is possible to semi-quantitatively estimate the coating composition for each sample; $2.5 \%$ APP /MTM contains 70\% APP and 30\% MTM while this proportion is basically inverted for 5\% APP/MTM which contains 30\% APP and 70\% MTM. Such difference might be related to the interactions between the negative phosphate groups of previously adsorbed APP and the positive edges of adsorbing MTM with the subsequent release of ammonium ions eventually employed for the production of stacked MTM layers. Such interaction would be possible only after the deposition of few (2-3) APP layer and 
thus be more apparent after eight deposition steps rather than four. However, while this explanation seems in accordance with XRD analyses (Figure 1f), in the absence of more detailed characterization the proposed adsorption mechanism remains a speculation. Undoubtedly, composition difference, along with the total coating add-on, could play an important role during cotton thermal degradation and flame retardancy.

\subsection{Thermal and Thermo-Oxidative Stability}

The thermal and thermo-oxidative stability of untreated and coated samples was assessed by thermogravimetric analyses in nitrogen and air, respectively. The aim is to obtain useful information concerning the effects of the deposited coating on the degradation pathways of cotton fabrics. First, only thermal degradation is evaluated in nitrogen environment. Figure 2 reports TG and dTG curves of untreated and treated samples and Table 1 collects temperature and weight data obtained from these analyses.

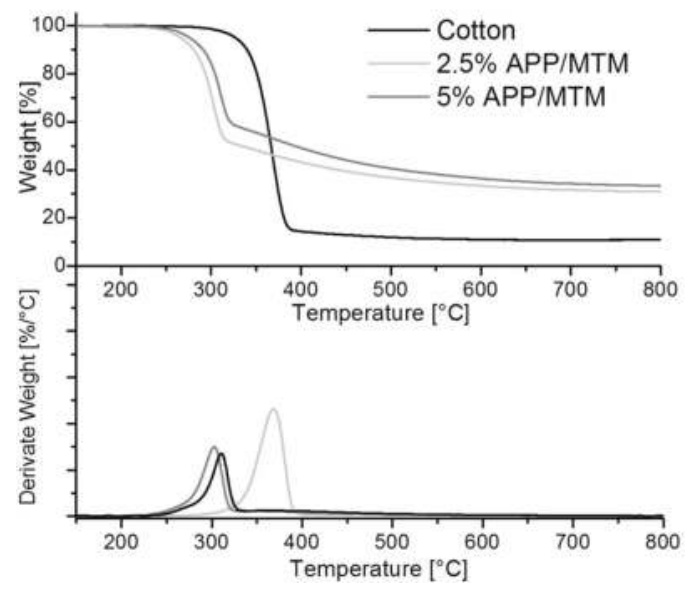

Figure 2. Thermogravimetric (TG) and derivative (dTG) curves of untreated and treated cotton fabrics in nitrogen.

Table 1. Thermogravimetric data of untreated and treated samples in nitrogen.

\begin{tabular}{cccc}
\hline Sample & $\boldsymbol{T}^{*}$ onset $\%\left({ }^{\circ} \mathrm{C}\right)$ & $T^{*}{ }_{\max }\left({ }^{\circ} \mathbf{C}\right)$ & Residue at $800{ }^{\circ} \mathbf{C}(\%)$ \\
\hline Cotton & 327 & 369 & 11 \\
$2.5 \%$ APP $/$ MTM & 266 & 301 & 31 \\
$5 \%$ APP $/$ MTM & 276 & 310 & 34 \\
\hline
\end{tabular}

The weight loss of cotton as a function of the temperature can be mostly related to the thermal degradation of cellulose, which is well known as well as its mechanism is already established. In nitrogen, the thermal decomposition of cellulose normally occurs by one step in between 300 and $400{ }^{\circ} \mathrm{C}$ and is the result of two competitive pathways, one involving the depolymerization of glycosyl units to volatile products (mainly levoglucosan, furan and furan derivatives) and the other involving the decomposition of the same units into thermally stable aromatic char (final residue evaluated at $800^{\circ} \mathrm{C}, 11 \%$ ) [36].

Treated fabrics still show a one-step thermal degradation. However, as clearly observable from Figure 2 the presence of the coating induces a strong anticipation in the degradation process, (see $T_{\text {onset5\% }}$ and $T_{\max }$ values in Table 1). This anticipation, more precisely defined "sensitization", 
is well known and associated to APP decomposition that producing phosphoric acid at high temperature favors the cellulose decomposition towards char formation $[37,38]$.

Interestingly there is an inverse proportionality between the coating add-on and the anticipation observed in TG curves; this can be explained by taking into account the different coating compositions, as previously observed from elemental analysis. Indeed, the P/Si ratio for 2.5\% APP/MTM treated samples is higher than for $5 \%$ sample, thus indicating a higher amount of APP within the coating with a consequent increase of the anticipating effect.

On the other hand, the effect of MTM, the presence of which is proportionally higher in $5 \%$ APP/MTM coatings, also has to be considered. Due to the lamellar chemical nature of these nanoparticles and the preferential orientation achieved through the deposition as observed in Figure 1, MTM can easily exert a barrier function towards the release of volatile products, thus balancing the anticipation ascribed to APP. This hypothesis is in agreement with the literature [35]. The latter combination of the two counterparts is beneficial and indeed provides the highest residue at $T_{\max }$ and at $800{ }^{\circ} \mathrm{C}$, nearly tripling the amount left by neat cotton (compare residues at $800^{\circ} \mathrm{C}$ in Table 1).

The thermo-oxidative stability of untreated and treated cotton fabrics has been assessed in air, thus evaluating the effects of an increasing temperature in an oxidizing environment. Figure 3 reports TG and dTG curves in air of untreated and treated samples and Table 2 collects temperature and weight data calculated from the plots.

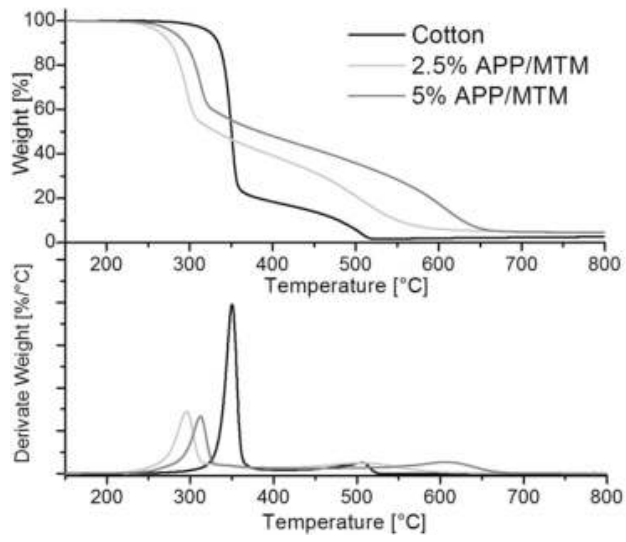

Figure 3. Thermogravimetric (TG) and derivative (dTG) curves of untreated and treated cotton fabrics in air.

Table 2. Thermogravimetric data of untreated and treated samples in air.

\begin{tabular}{cccccc}
\hline Sample & $T^{*}{ }_{\text {onset5 } \%}\left({ }^{\circ} \mathrm{C}\right)$ & $T^{*}{ }_{\max \mathbf{1}}\left({ }^{\circ} \mathrm{C}\right)$ & $T^{*}{ }_{\max 2}\left({ }^{\circ} \mathrm{C}\right)$ & Residue at $\mathbf{4 0 0}{ }^{\circ} \mathbf{C}(\%)$ & Residue at $800{ }^{\circ} \mathbf{C}(\%)$ \\
\hline Cotton & 324 & 350 & 506 & 18 & 2 \\
$2.5 \%$ & 263 & 296 & 508 & 39 & 4 \\
APP/MTM & 280 & 313 & 607 & 48 & 5 \\
$5 \%$ APP /MTM & \multicolumn{5}{c}{ From derivative TG curves. }
\end{tabular}

The thermal oxidation of cotton normally takes place in two definite steps: the first one between 300 and $400^{\circ} \mathrm{C}$ is related to the formation of both volatiles and al aliphatic char $(18 \%)$, while the second is due to the almost complete oxidation of the char with the release of $\mathrm{CO}$ and $\mathrm{CO}_{2}$ [39-41]. Similar to what observed in nitrogen, treated fabrics showed anticipation inversely proportional to the coating

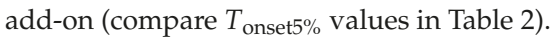


Of particular interest is the coating shielding effect towards oxygen during the second degradation step; here, the $5 \%$ APP / MTM sample maintains $40 \%$ residue up to $550{ }^{\circ} \mathrm{C}$ and achieves the highest delay in the second degradation step, as confirmed from $\mathrm{T}_{\max 2}$ value that is shifted to $600{ }^{\circ} \mathrm{C}$ with an increase of $100{ }^{\circ} \mathrm{C}$ with respect to unmodified cotton (compare $T_{\max 2}$ values in Table 2). This effect can be ascribed to the formation of a protective coating capable of greatly postponing and slowing down the oxidation of the residue produced during the first degradation step.

\subsection{Horizontal Flame Spread Tests}

Flammability test in horizontal configuration has been employed to investigate the reaction of untreated and treated fabrics to a direct flame application. This test assesses the propensity of a material to initiate a fire and represents a fundamental test in the field of flame retardancy. Figure 4 reports snapshots of uncoated and coated fabrics during the test and Table 3 reports the collected parameters.
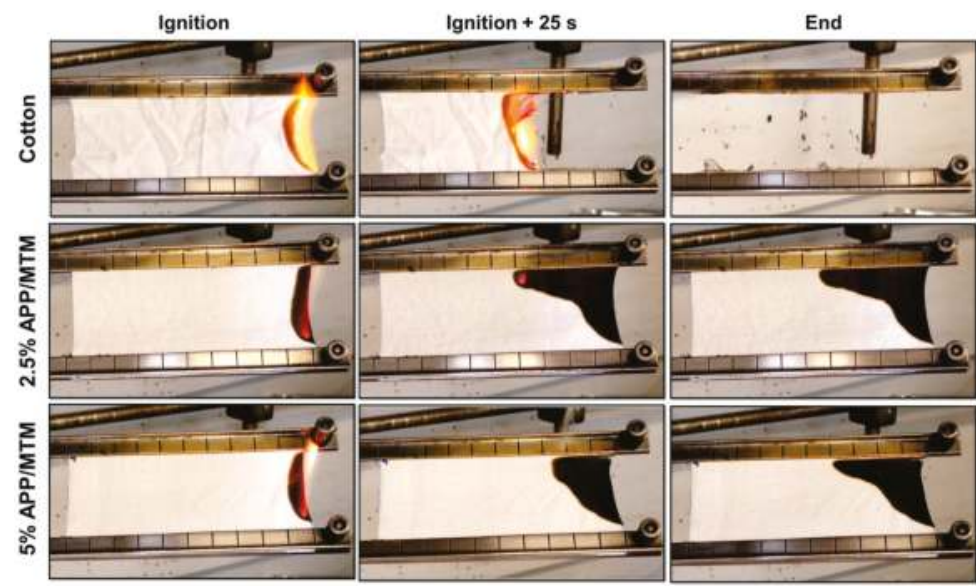

Figure 4. Snapshot of flame-spread tests of untreated and treated cotton fabrics.

Table 3. Flammability data from horizontal flame tests of untreated and treated cotton fabrics.

\begin{tabular}{cccc}
\hline Sample & Combustion Rate $\pm \sigma(\mathrm{mm} / \mathbf{s})$ & Afterglow & Residue $\pm \sigma(\%)$ \\
\hline Cotton & $1.7 \pm 0.04$ & Yes & 0 \\
$2.5 \%$ APP/MTM & $1.6 \pm 0.30$ & No & $78 \pm 5$ \\
$5 \%$ APP/MTM & $1.3 \pm 0.08$ & No & $85 \pm 2$ \\
\hline
\end{tabular}

Upon flame application, untreated cotton immediately ignites and burns with flames that, fed by the combustible degradation products of cotton, spread towards the opposite site of sample at an almost constant speed. As the flame reaches the end of the sample, it vanishes and the remaining charred cotton fibers are further consumed by a solid-state oxidation characterized by red incandescence known as afterglow. The high temperatures reached during the afterglow are still capable of spreading the fire to other ignitable materials, thus posing an additional, although smaller than flames, risk to safety.

The coating can significantly modify the burning behavior of cotton. Indeed, both treatments were able to stop the propagation of the flame achieving a self-extinguishing behavior, thus resulting in very high residues. In detail, upon flame propagation the coating action as protective barrier and char formation enhancer reduces the release of volatile combustible products; by this way, the combustion cannot be sustained anymore and the flame gradually reduces in size being confined to a smaller 
and smaller region where, eventually, it vanishes (see Figure 4). Furthermore, any subsequent flame application cannot ignite the sample again.

\subsection{Cone Calorimetry}

Cone calorimetry has been employed in order to evaluate the reaction of uncoated and coated samples to the exposure to a heat flux. The latter is controlled in order to have heat flux values typical of early stage developing fires (i.e., $35 \mathrm{~kW} / \mathrm{m}^{2}$ ) [42]. When exposed to the heat flux, samples start to degrade releasing combustible gases that are ignited by a spark positioned above the sample. The time required for reaching ignition is normally referred as time to ignition (TTI). Then, flaming combustion starts and the heat released is calculated by evaluating the oxygen consumed during the process.

Heat Release Rate (HRR) plots of untreated and treated samples are reported in Figure 5 together with a schematization of sample behavior during the test. Table 4 reports the collected numerical data.

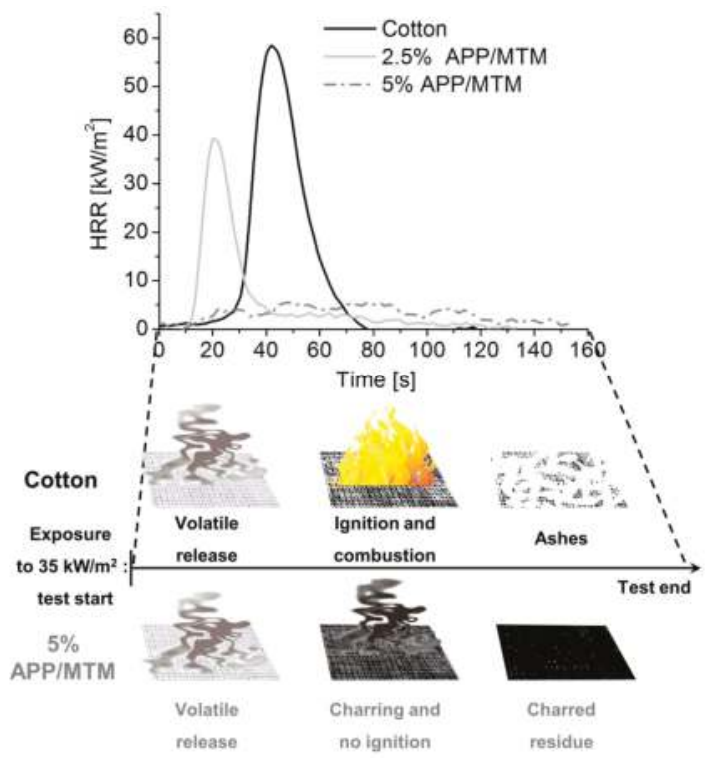

Figure 5. Average Heat release rate (HRR) plots of untreated and treated cotton during cone calorimetry tests and schematic representation of sample behavior under testing.

Table 4. Cone calorimetry data of untreated and treated samples.

\begin{tabular}{cccccc}
\hline Sample & TTI $\pm \sigma(\mathbf{s})$ & pkHRR $\pm \sigma\left(\mathbf{k W} / \mathbf{m}^{2}\right)$ & THR $\pm \sigma\left(\mathbf{M J} / \mathbf{m}^{2}\right)$ & TSR $\pm \sigma\left(\mathbf{m}^{2} / \mathbf{m}^{2}\right)$ & Residue $(\%)^{2}$ \\
\hline Cotton & $36 \pm 2$ & $61 \pm 4$ & $1.0 \pm 0.1$ & $25 \pm 8$ & 0 \\
$2.5 \%$ & $22 \pm 5$ & $38 \pm 7$ & $0.38 \pm 0.05$ & $13 \pm 4$ & 13 \\
APP $/$ MTM & N.A. ${ }^{*}$ & N.A. ${ }^{*}$ & N.A. ${ }^{*}$ & $40 \pm 6$ & 19 \\
\hline
\end{tabular}

* Parameters related to combustion are not available, as samples did not ignite during test.

Unmodified cotton rapidly ignites after $36 \mathrm{~s}$ with a quick combustion and an average pkHRR of $61 \mathrm{~kW} / \mathrm{m}^{2}$, without leaving any residue at the end. The $2.5 \%$ APP/MTM shows an anticipation in ignition as TTI is reduced to $22 \mathrm{~s}$; this can be ascribed to the presence of APP that, similar to what observed in TGA, releases phosphoric acid that favors cellulose dehydration, thus resulting in an early production of volatiles with subsequent early ignition. This phenomenon is not considered detrimental, as by this way the production of charred residue is favored despite volatile release [29]. 
Thus, the total heat release and combustion kinetics are significantly reduced, as demonstrated by HRR plots and THR and pkHRR values reported in Table 4.

Surprisingly, 5\% APP/MTM samples showed no ignition at all during this test. Upon exposure to the heat flux, samples start releasing volatile products while simultaneously producing char, as clearly observable by a change in color (from white to black). The latter process, improved by the presence of APP, is combined with the barrier effect exerted by MTM that slows down the gas release. Therefore, by the combination of these two flame retardant actions, the released combustible volatile products are not able to reach the concentration needed for ignition, as schematically depicted in Figure 5. This is also confirmed by TSR (Total Smoke Release) values that show an increase for non-igniting samples (Table 4).

All treated fabrics yielded a compact and coherent residue at the end of the test (see Figure S4 in Supplementary Materials) that, at the higher coating add-on, maintained the original shape of the fabric. Interestingly, it was possible to handle the residues without damaging them (e.g., they could be bent by $180^{\circ}$ without breaking), thus indicating that they partially maintained the original mechanical properties.

\subsection{Residue Analysis and Coating Mechanism}

The residues collected at the end of cone calorimetry tests have been analyzed using SEM, FT-IR/ATR and Raman spectroscopies in order to obtain information useful for interpreting the results obtained during flammability and cone calorimetry tests. Figure 6 reports SEM observations performed on 5\% APP/MTM, and IR and Raman spectra of both 2.5\% APP/MTM and 5\% APP/MTM residues.
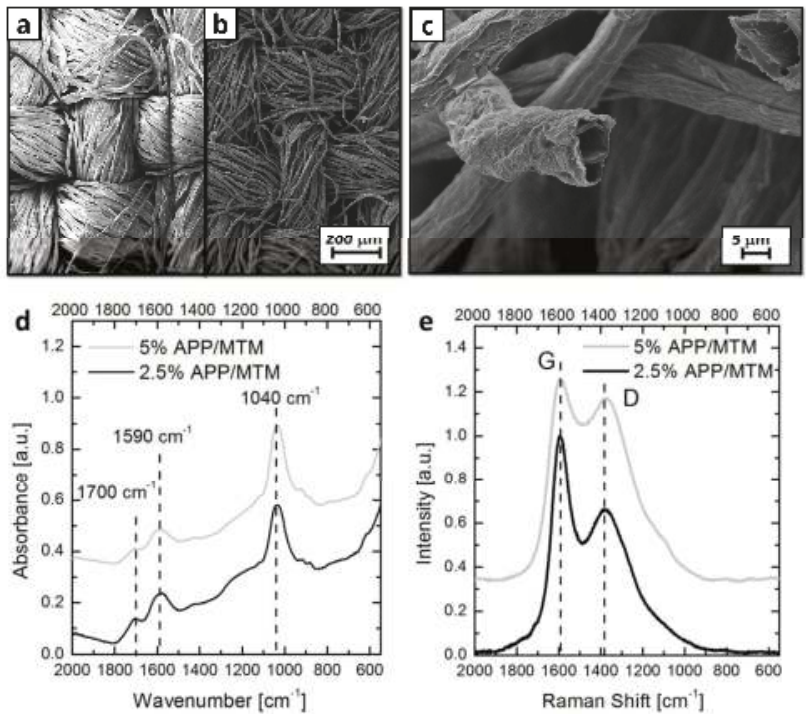

Figure 6. Analyses on residues after cone calorimetry: low magnification SEM (Scanning Electron Microscopy) micrograph performed on unburned (a) and burned (b) areas of 5\% APP/MTM; detail of 5\% APP/MTM fibers after combustion (c); and FT-IR/ATR and Raman spectra (d,e). As observable from SEM micrograph, the residues were able to maintain the original texture of the fabric while the single fibers appear damaged and shrunk with respect to the unburned sample (compare Figure 6a,b). In addition, from a closer observation, the single fibers appear to be surrounded by a sort of expanded structure that completely covers them. This structure is resulting from the exposure of the original APP/MTM coating to a heat flux; EDS analysis confirms the composition by pointing out the presence of both P and Si elements with C being the main component (see Figure S5 in Supplementary Materials). 
IR and Raman spectroscopies provide additional information concerning the structure of the carbonaceous structure. Indeed, IR signals related to aromatic char can be found at $1590 \mathrm{~cm}^{-1}$ while the signal at $1040 \mathrm{~cm}^{-1}$ can be associated to Si-O-Si bonds in MTM nanoplatelets [43]. Furthermore, the presence of a weak peak at $1700 \mathrm{~cm}^{-1}$ associated to $\mathrm{C}=\mathrm{O}$ bonds indicates the partial oxidation of the residues.

Raman spectroscopy gives complementary details concerning the nature of the produced char. As reported in Figure 6e, both residues show two characteristic peaks (namely, G and D bands at 1590 and $1350 \mathrm{~cm}^{-1}$, respectively) normally associated to polyaromatic hydrocarbons, further confirming the aromatic nature of the structures produced during combustion [44,45].

Basing on the achieved results and the mentioned above characterization, it is possible to devise the coating mechanism. To this aim, the coating reaction to heat or flame application is reported in Figure 7 where a FE-SEM micrograph of coating (5\% APP/MTM)) before combustion (Figure 7a), SEM micrograph of expanded coating (namely, 5\% APP/MTM) after combustion (Figure 7b) and a schematization of the coating flame retardant action (Figure 7c) are depicted.
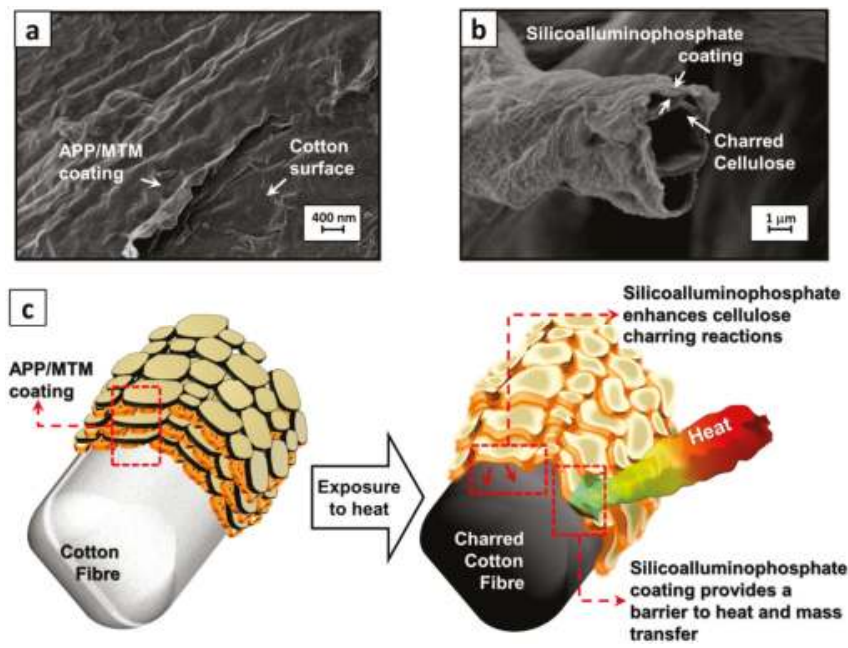

Figure 7. Coating reaction to heat or flame application: FE-SEM micrograph of coating (5\% APP/MTM)) before combustion (a); SEM micrograph of expanded coating (5\% APP/MTM) after combustion (b); and schematization of the coating flame retardant action (c).

Upon exposure to a heat flux or flame, APP starts to degrade releasing ammonia and producing phosphoric and polypohosphoric acid [38]. Ammonia swells the MTM stacks deposited within the coating while polypohosphoric acid simultaneously promotes the char formation of cotton, as well known from the literature [37]. In addition, besides reacting with cotton for enhanced char production, APP can also react with MTM nanoplatelets; indeed, clay can favor the dissociation of APP and the phosphoric acid generated can react with MTM nanoplatelets joining them together with the production of a silicoalluminophosphate [30,31]. In the case of the coating, the inorganic expanded structure can also act as physical barrier slowing down mass, oxygen and heat transfer between the flame and the substrate.

\section{Conclusions}

In the present manuscript, a new concept of completely inorganic intumescent flame retardant coating deposited by a multistep adsorption process from diluted water-based ammonium polyphosphate solutions and sodium montmorillonite suspensions has been explored, using cotton as 
model substrate. The coating covers the surface of each cotton fiber resulting in very thin coatings with thickness below $50 \mathrm{~nm}$ where montmorillonite stacks are embedded in a continuous matrix of ammonium polyphosphate. This coating efficiently enhanced the char production from cotton fibers, as observed by thermogravimetric analyses in both nitrogen and air, with impressive results achieved during combustion tests. The coating resulting in a total add-on of $5 \%$ with respect to the original fabric mass achieved self-extinguishing during horizontal flame spread tests and prevented ignition of the fabrics when exposed to $35 \mathrm{~kW} / \mathrm{m}^{2}$ during cone calorimetry test. The flame retardant action has been ascribed to the formation of a silicoalluminophosphate expanded structure capable of on the one hand enhancing the cellulose char-forming ability and on the other hand providing a physical barrier to mass, oxygen and heat transfer between the flame and the substrate. These results demonstrate the potentialities of the concept proposed and developed within this paper and provide a starting point for the further improvements of the coating performances. For instance, the coating stability and durability need to be improved by employing different cross-linking strategies if an application as protective treatment for fabrics has to be foreseen.

Supplementary Materials: The following are available online at http:/ /www.mdpi.com/2073-4360/8/12/430/s1, Figure S1: Images of MTM (a), APP (b) and mixture of APP/MTM (c) after irradiation under $35 \mathrm{~kW} / \mathrm{m}^{2}$ heat flux in a cone calorimeter. Figure S2: FE-SEM micrographs of defects in 2.5\% APP/MTM samples. Figure S3: EDS analyses performed on 2.5\% APP /MTM and 5\% APP/MTM samples. Figure S4: Images of residues collected at the end of cone calorimetry tests: (a) untreated cotton, (b) $2.5 \%$ APP/MTM and (c) 5\% APP/MTM samples. Figure S5: EDS analyses performed on 5\% APP/MTM residues collected at the end of cone calorimetry tests.

Acknowledgments: The Authors want to thank Fabio Cuttica (for cone calorimetry tests), Alessandro Di Blasio (for SEM analyses) and Mauro Raimondo (for FE-SEM analyses).

Author Contributions: The present manuscript was written with the equal contribution of the two Authors. J.A. and F.C. conceived and designed the experiments; F.C. performed the experiments and analyzed the data; J.A. and F.C. wrote the paper.

Conflicts of Interest: The authors declare no conflict of interest.

\section{References}

1. Birnbaum, L.S.; Staskal, D.F. Brominated flame retardants: Cause for concern? Environ. Health Perspect. 2003, 112, 9-17. [CrossRef]

2. Xiao, H.; Shen, L.; Su, Y.; Barresi, E.; Dejong, M.; Hung, H.; Lei, Y.D.; Wania, F.; Reiner, E.J.; Sverko, E.; et al. Atmospheric concentrations of halogenated flame retardants at two remote locations: The Canadian high arctic and the Tibetan plateau. Environ. Pollut. 2012, 161, 154-161. [CrossRef] [PubMed]

3. Barcelo, D.; Kostianoy, A.G. Handbook environmental chemistry. In Brominated Flame Retardants; Heidelberg, S.-V.B., Ed.; Springer: New York, NY, USA, 2011; Volume 16.

4. Stieger, G.; Scheringer, M.; Ng, C.A.; Hungerbuhler, K. Assessing the persistence, bioaccumulation potential and toxicity of brominated flame retardants: Data availability and quality for 36 alternative brominated flame retardants. Chemosphere 2014, 116, 118-123. [CrossRef] [PubMed]

5. Ray, S.S.; Okamoto, M. Polymer/layered silicate nanocomposites: A review from preparation to processing. Prog. Polym. Sci. 2003, 28, 1539-1641.

6. Kiliaris, P.; Papaspyrides, C. Polymer/layered silicate (clay) nanocomposites: An overview of flame retardancy. Prog. Polym. Sci. 2010, 35, 902-958. [CrossRef]

7. Fernandes, N.J.; Akbarzadeh, J.; Peterlik, H.; Giannelis, E.P. Synthesis and properties of highly dispersed ionic silica-poly(ethylene oxide) nanohybrids. ACS Nano 2013, 7, 1265-1271. [CrossRef] [PubMed]

8. Priolo, M.A.; Holder, K.M.; Guin, T.; Grunlan, J.C. Recent advances in gas barrier thin films via layer-by-layer assembly of polymers and platelets. Macromol. Rapid. Commun. 2015, 36, 866-879. [CrossRef] [PubMed]

9. Malucelli, G.; Carosio, F.; Alongi, J.; Fina, A.; Frache, A.; Camino, G. Materials engineering for surface-confined flame retardancy. Mat. Sci. Eng. R 2014, 84, 1-20. [CrossRef]

10. Laufer, G.; Kirkland, C.; Cain, A.A.; Grunlan, J.C. Clay-Chitosan nanobrick walls: Completely renewable gas barrier and flame-retardant nanocoatings. ACS Appl. Mater. Interfaces 2012, 4, 1643-1649. [CrossRef] [PubMed] 
11. Carosio, F.; Colonna, S.; Fina, A.; Rydzek, G.; Hemmerle, J.; Jierry, L.; Schaaf, P.; Boulmedais, F. Efficient gas and water vapor barrier properties of thin poly(lactic acid) packaging films: Functionalization with moisture resistant nafion and clay multilayers. Chem. Mater. 2014, 26, 5459-5466. [CrossRef]

12. Alongi, J.; Tata, J.; Carosio, F.; Rosace, G.; Frache, A.; Camino, G. A comparative analysis of nanoparticle adsorption as fire-protection approach for fabrics. Polymers 2015, 7, 47-68. [CrossRef]

13. Alongi, J.; Carosio, F.; Kiekens, P. Recent advances in the design of water based-flame retardant coatings for polyester and polyester-cotton blends. Polymers 2016, 8, 357-380. [CrossRef]

14. Decher, G.; Hong, J.D.; Schmitt, J. Buildup of ultrathin multilayer films by a self-assembly process: III. Consecutively alternating adsorption of anionic and cationic polyelectrolytes on charged surfaces. Thin Solid Films 1992, 210-211, 831-835. [CrossRef]

15. Decher, G. Fuzzy nanoassemblies: Toward layered polymeric multicomposites. Science 1997, 277, 1232-1237. [CrossRef]

16. Laufer, G.; Carosio, F.; Martinez, R.; Camino, G.; Grunlan, J.C. Growth and fire resistance of colloidal silica-polyelectrolyte thin film assemblies. J. Colloid. Interface Sci. 2011, 356, 69-77. [CrossRef] [PubMed]

17. Carosio, F.; Cuttica, F.; Di Blasio, A.; Alongi, J.; Malucelli, G. Layer by layer assembly of flame retardant thin films on closed cell pet foams: Efficiency of ammonium polyphosphate versus DNA. Polym. Degrad. Stab. 2015, 113, 189-196. [CrossRef]

18. Carosio, F.; Di Blasio, A.; Alongi, J.; Malucelli, G. Layer by layer nanoarchitectures for the surface protection of polycarbonate. Eur. Polym. J. 2013, 49, 397-404. [CrossRef]

19. Apaydin, K.; Laachachi, A.; Ball, V.; Jimenez, M.; Bourbigot, S.; Toniazzo, V.; Ruch, D. Polyallylaminemontmorillonite as super flame retardant coating assemblies by layer-by layer deposition on polyamide. Polym. Degrad. Stab. 2013, 98, 627-634. [CrossRef]

20. Fina, A.; Cuttica, F.; Camino, G. Ignition of polypropylene/montmorillonite nanocomposites. Polym. Degrad. Stab. 2012, 97, 2619-2626. [CrossRef]

21. Cain, A.A.; Nolen, C.R.; Li, Y.C.; Davis, R.; Grunlan, J.C. Phosphorous-filled nanobrick wall multilayer thin film eliminates polyurethane melt dripping and reduces heat release associated with fire. Polym. Degrad. Stab. 2013, 98, 2645-2652. [CrossRef]

22. Alongi, J.; Carosio, F.; Malucelli, G. Layer by layer complex architectures based on ammonium polyphosphate, chitosan and silica on polyester-cotton blends: Flammability and combustion behaviour. Cellulose 2012, 19, 1041-1050. [CrossRef]

23. Holder, K.M.; Huff, M.E.; Cosio, M.N.; Grunlan, J.C. Intumescing multilayer thin film deposited on clay-based nanobrick wall to produce self-extinguishing flame retardant polyurethane. J. Mater. Sci. 2015, 50, 2451-2458. [CrossRef]

24. Carosio, F; Alongi, J. Ultra-fast layer-by-layer approach for depositing flame retardant coatings on flexible pu foams within seconds. ACS Appl. Mater. Interfaces 2016, 8, 6315-6319. [CrossRef] [PubMed]

25. Carosio, F.; Alongi, J.; Malucelli, G. Flammability and combustion properties of ammonium polyphosphate-/ poly(acrylic acid)-based layer by layer architectures deposited on cotton, polyester and their blends. Polym. Degrad. Stab. 2013, 98, 1626-1637. [CrossRef]

26. Alongi, J.; Han, Z.; Bourbigot, S. Intumescence: Tradition versus novelty. A comprehensive review. Prog. Polym. Sci. 2015, 51, 28-73. [CrossRef]

27. Carosio, F.; Alongi, J.; Malucelli, G. Layer by layer ammonium polyphosphate-based coatings for flame retardancy of polyester-cotton blends. Carbohydr. Polym. 2012, 88, 1460-1469. [CrossRef]

28. Li, Y.C.; Mannen, S.; Morgan, A.B.; Chang, S.C.; Yang, Y.H.; Condon, B.; Grunlan, J.C. Intumescent all-polymer multilayer nanocoating capable of extinguishing flame on fabric. Adv. Mater. 2011, 23, 3926-3931. [CrossRef] [PubMed]

29. Carosio, F.; Fontaine, G.; Alongi, J.; Bourbigot, S. Starch-based layer by layer assembly: Efficient and sustainable approach to cotton fire protection. ACS Appl. Mater. Interfaces 2015, 7, 12158-12167. [CrossRef] [PubMed]

30. Yi, D.; Yang, R. Ammonium polyphosphate/montmorillonite nanocompounds in polypropylene. J. Appl. Polym. Sci. 2010, 118, 834-840. [CrossRef]

31. Ma, H.; Tong, L.; Xu, Z.; Fang, Z. Intumescent flame retardant-montmorillonite synergism in ABS nanocomposites. Appl. Clay Sci. 2008, 42, 238-245. [CrossRef] 
32. Tata, J.; Alongi, J.; Carosio, F.; Frache, A. Optimization of the procedure to burn textile fabrics by cone calorimeter: Part I. Combustion behavior of polyester. Fire Mater. 2011, 35, 397-409. [CrossRef]

33. Norrish, K. The swelling of montmorillonite. Discuss. Faraday Soc. 1954, 18, 120-134. [CrossRef]

34. Zheng, Y.; Zaoui, A. How water and counterions diffuse into the hydrated montmorillonite. Solid State Ion. 2011, 203, 80-85. [CrossRef]

35. Carosio, F.; Kochumalayil, J.; Cuttica, F.; Camino, G.; Berglund, L. Oriented clay nanopaper from biobased components-mechanisms for superior fire protection properties. ACS Appl. Mater. Interfaces 2015, 7, 5847-5856. [CrossRef] [PubMed]

36. Alongi, J.; Camino, G.; Malucelli, G. Heating rate effect on char yield from cotton, poly(ethylene terephthalate) and blend fabrics. Carbohydr. Polym. 2013, 92, 1327-1334. [CrossRef] [PubMed]

37. Davies, P.J.; Horrocks, A.R.; Alderson, A. The sensitisation of thermal decomposition of ammonium polyphosphate by selected metal ions and their potential for improved cotton fabric flame retardancy. Polym. Degrad. Stab. 2005, 88, 114-122. [CrossRef]

38. Camino, G.; Costa, L.; Trossarelli, L. Study of the mechanism of intumescence in fire retardant polymers.3. Effect of urea on the ammonium polyphosphate pentaerythritol system. Polym. Degrad. Stab. 1984, 7, 221-229. [CrossRef]

39. Price, D.; Horrocks, A.R.; Akalin, M.; Faroq, A.A. Influence of flame retardants on the mechanism of pyrolysis of cotton (cellulose) fabrics in air. J. Anal. Appl. Pyrolysis 1997, 40-41, 511-524. [CrossRef]

40. Mamleev, V.; Bourbigot, S.; Le Bras, M.; Yvon, J. The facts and hypotheses relating to the phenomenological model of cellulose pyrolysis interdependence of the steps. J. Anal. Appl. Pyrolysis 2009, 84, 1-17. [CrossRef]

41. Kandola, B.K.; Horrocks, A.R.; Price, D.; Coleman, G.V. Flame-retardant treatments of cellulose and their influence on the mechanism of cellulose pyrolysis. J. Macromol. Sci. RMC 1996, C36, 721-794. [CrossRef]

42. Schartel, B.; Hull, T.R. Development of fire-retarded materials-Interpretation of cone calorimeter data. Fire Mater. 2007, 31, 327-354. [CrossRef]

43. Soares, S.; Camino, G.; Levchik, S. Comparative-study of the thermal-decomposition of pure cellulose and pulp paper. Polym. Degrad. Stab. 1995, 49, 275-283. [CrossRef]

44. Carosio, F.; Alongi, J. Influence of layer by layer coatings containing octapropylammonium polyhedral oligomeric silsesquioxane and ammonium polyphosphate on the thermal stability and flammability of acrylic fabrics. J. Anal. Appl. Pyrolysis 2016, 119, 114-123. [CrossRef]

45. Ferrari, A.C.; Basko, D.M. Raman spectroscopy as a versatile tool for studying the properties of graphene. Nat. Nanotechnol. 2013, 8, 235-246. [CrossRef] [PubMed]

(C) 2016 by the authors. Licensee MDPI, Basel, Switzerland. This article is an open access article distributed under the terms and conditions of the Creative Commons Attribution (CC BY) license (http:/ / creativecommons.org/licenses/by/4.0/). 


\title{
Article \\ Polysarcosine-Based Lipids: From Lipopolypeptoid Micelles to Stealth-Like Lipids in Langmuir Blodgett Monolayers
}

\author{
Benjamin Weber ${ }^{1}$, Christine Seidl ${ }^{1}$, David Schwiertz ${ }^{1}$, Martin Scherer ${ }^{1}$, Stefan Bleher ${ }^{2}$, \\ Regine Süss ${ }^{2}$ and Matthias Barz ${ }^{1, *}$ \\ 1 Institute of Organic Chemistry, Johannes Gutenberg University of Mainz, Duesbergweg 10-14, 55128 Mainz, \\ Germany; b.weber@uni-mainz.de (B.W.); cseidl@students.uni-mainz.de (C.S.); \\ daschwie@students.uni-mainz.de (D.S.); m.scherer@uni-mainz.de (M.S.) \\ 2 Department of Pharmaceutical Technology and Biopharmacy, Institute of Pharmaceutical Sciences, \\ Albert Ludwigs University of Freiburg, Sonnenstraße 5, 79104 Freiburg im Breisgau, Germany; \\ stefan.bleher@pharmazie.uni-freiburg.de (S.B.); regine.suess@pharmazie.uni-freiburg.de (R.S.) \\ * Correspondence: barz@uni-mainz.de; Tel.: +49-6131-392-5468
}

Academic Editor: Alexander Böker

Received: 17 October 2016; Accepted: 2 December 2016; Published: 9 December 2016

\begin{abstract}
Amphiphiles and, in particular, PEGylated lipids or alkyl ethers represent an important class of non-ionic surfactants and have become key ingredients for long-circulating ("stealth") liposomes. While poly-(ethylene glycol) (PEG) can be considered the gold standard for stealth-like materials, it is known to be neither a bio-based nor biodegradable material. In contrast to PEG, polysarcosine (PSar) is based on the endogenous amino acid sarcosine ( $N$-methylated glycine), but has also demonstrated stealth-like properties in vitro, as well as in vivo. In this respect, we report on the synthesis and characterization of polysarcosine based lipids with $\mathrm{C}_{14}$ and $\mathrm{C}_{18}$ hydrocarbon chains and their end group functionalization. Size exclusion chromatography (SEC) and matrix-assisted laser desorption/ionization time-of-flight mass spectrometry (MALDI-TOF MS) analysis reveals that lipopeptoids with a degree of polymerization between 10 and 100, dispersity indices around 1.1, and the absence of detectable side products are directly accessible by nucleophilic ring opening polymerization (ROP). The values for the critical micelle concentration for these lipopolymers are between 27 and $1181 \mathrm{mg} / \mathrm{L}$ for the ones with $C_{18}$ hydrocarbon chain or even higher for the $C_{14}$ counterparts. The lipopolypeptoid based micelles have hydrodynamic diameters between 10 and $25 \mathrm{~nm}$, in which the size scales with the length of the PSar block. In addition, $\mathrm{C}_{18} \mathrm{PSar}_{50}$ can be incorporated in 1,2-distearoyl-sn-glycero-3-phosphocholine (DSPC) monolayers up to a polymer content of 3\%. Cyclic compression and expansion of the monolayer showed no significant loss of polymer, indicating a stable monolayer. Therefore, lipopolypeptoids can not only be synthesized under living conditions, but my also provide a platform to substitute PEG-based lipopolymers as excipients and/or in lipid formulations.
\end{abstract}

Keywords: polysarcosine; polypeptoids; surfactants; lipids; NCA polymerization; PSarcosinylated lipids

\section{Introduction}

Amphiphilic molecules and polymers are commonly applied to lower the surface tension (or interfacial tension) between two liquids or between a liquid and a solid, which enables their use as detergents, wetting agents, emulsifiers, foaming agents, and dispersants [1,2]. From a structural point of view, these polymers can be divided into two classes, which are characterized by the relative distribution of hydrophilic and lipophilic units. Macromolecules based on intrinsically amphiphilic 
repeating units are summarized as "polysoaps", whereas polymers with strictly separated parts are called "macrosurfactants" [3-6]. These macrosurfactants are commonly amphiphilic block copolymers or lipopolymers, in which a hydrocarbon chain of 12-18 units is attached to a hydrophilic polymer. With respect to sustainability, bio-based amphiphiles, such as lipopeptides, have gained pronounced attention as they are based on renewable raw materials [7]. This enormous potential was already recognized half a century ago [8-10] and, thus, several amino acid or peptide based amphiphiles have been investigated, in which fatty acid chains, as well as amino acids or peptides, can vary in composition and length [11].

In addition, PEGylated lipids or alkyl ethers represent an important class of non-ionic lipopolymers and are key ingredients for the preparation of long-circulating liposomes, since a significant step in the development of long-circulating liposomes came with the incorporation of the synthetic polymer poly-(ethylene glycol) (PEG) in liposome compositions. The presence of PEG on the surface of the liposomal carriers has been shown to extend blood-circulation time while reducing mononuclear phagocyte system uptake (stealth liposomes). Despite the enormous achievements of PEGylated lipids, several groups have reported immune responses towards PEG and PEGylated lipids, leading to the accelerated blood clearance (ABC) phenomenon [12-14]. Moreover, PEG is not degradable in vivo and relies on complete excretion to avoid storage diseases [15]. Consequently, the finding of alternatives to PEG is a growing field of research [16]. Among various PEG surrogates, polypeptides and polypeptoids are attracting more and more attention [16-18]. The polypeptoid polysarcosine (PSar) seems to be particularly interesting because it is, on one hand, based on the endogenous $N$-substituted amino acid, sarcosine ( $N$-methylated glycine), and on the other hand, sarcosine can be easily synthesized by a simple nucleophilic substitution reaction of bromo- or chloroacetic acid and methylamine [19]. Furthermore, PSar can be synthesized under living conditions from the corresponding $\alpha$-amino acid $N$-carboxy anhydride (NCA) [19-21] and has already demonstrated possessing stealth-like properties comparable to PEG [18,22-26]. Interestingly, lipopeptides, as non-ionic and bio-based systems, have been practically overlooked. So far only Gallot and coworkers have reported on the synthesis of PSar-based lipopolymers (lipopeptoids). In 1986 they reported the synthesis of lipopeptoids using aliphatic amines to initiate the ring opening polymerization (ROP) of the Sar NCA in chloroform $[27,28]$. Surprisingly, they had to fractionate the final lipopolypeptoid yielding different fractions with degrees of polymerization from 10 to 60 . Due to the living nature of Sar NCA ROP, one would expect that such degrees of polymerization can be directly obtained by adjusting the monomer to initiator ratio. To validate our expectation, we carried out the synthesis of lipopolypeptoids based on either tetradecylamine $\left(C_{14}\right)$ or stearylamine $\left(C_{18}\right)$.

In this work, we report the synthesis and end group functionalization of PSar based lipopolypeptoids with $\mathrm{C}_{14}$ and $\mathrm{C}_{18}$ hydrocarbon tails. The lipopolypeptoids have a PSar block with chain lengths $\left(X_{n}\right)$ from 10-100. Furthermore, we introduce a synthetic pathway, which allows polymerization of such systems on $50-100 \mathrm{~g}$ scale. The final lipopolymers are characterized by ${ }^{1} \mathrm{H}$ NMR, ${ }^{1} \mathrm{H}$-DOSY NMR, SEC and MALDI-TOF mass spectrometry to ensure the living nature of the ring opening polymerization. We also report on the critical micelle concentration (CMC) of the synthesized systems, characterize the aggregates by dynamic light scattering, investigate cellular toxicities, and report on the incorporation of 1, 2, and $3 \mathrm{~mol} \%$ of PSar45-stearylamine into Langmuir-Blodgett monolayers of 1,2-distearoyl-sn-glycero-3-phosphocholine (DSPC).

\section{Materials and Methods}

$n$-Hexane was distilled from $\mathrm{Na} / \mathrm{K}$ and ethyl acetate from $\mathrm{CaH}_{2}$. Dimethylformamide (DMF) was purchased from Acros Organics (Geel, Belgium) and dried over $\mathrm{BaO}$ and molecular sieves ( $3 \AA$ ), fractionally distilled under vacuum at $40{ }^{\circ} \mathrm{C}$ and stored at $-80^{\circ} \mathrm{C}$ under the exclusion of light. Prior to use, DMF was degassed in vacuum to remove traces of dimethylamine. Hexafluoroisopropanol (HFIP) was purchased from Fluorochem (Hadfield Derbyshire, UK). Millipore water was prepared by a MILLI-Q ${ }^{\circledR}$ Reference $\mathrm{A}^{+}$System (Darmstadt, Germany). Octadecylamine and was purchased from Fluka 
(St. Gallen, Switzerland) and was dried at $40^{\circ} \mathrm{C}$ under vacuum $\left(1 \times 10^{-3} \mathrm{mbar}\right)$ for $24 \mathrm{~h}$. Diphosgene was purchased from Alfa Aesar (Ward Hill, MA, USA) and deuterated solvents from Deutero GmbH (Kastellaun, Germany). Other chemicals were purchased from Sigma-Aldrich (Taufkirchen, Germany) and used as received unless otherwise stated. 1,2-distearoyl-sn-glycero-3-phosphocholine (DSPC) was purchased from Avanti Polar Lipids (Alabaster, Al, USA) and used without purification. Roswell Park Memorial Institute (RPMI) cell medium and FCS was purchased from Merck Millipore (Darmstadt, Germany). HeLa cells were obtained from DSMZ (German Collection of Microorganisms and Cell Cultures, Braunschweig, Germany).

${ }^{1} \mathrm{H}$ NMR spectra were recorded on a Bruker (Billerica, MA, USA) AC 400 at a frequency of $400 \mathrm{MHz}$ respectively. Two-dimensional NMR spectra as ${ }^{1} \mathrm{H}$ DOSY were recorded on a Bruker Avance III HD 400 at $400 \mathrm{MHz}$. All spectra were recorded at room temperature $\left(25^{\circ} \mathrm{C}\right)$ and calibrated using the solvent signals. Melting points were measured using a Mettler FP62 melting point apparatus at a heating rate of $2.5^{\circ} \mathrm{C} \cdot \mathrm{min}^{-1}$. Gel permeation chromatography (GPC) was performed with hexafluoroisopropanol (HFIP) containing $3 \mathrm{~g} \cdot \mathrm{L}^{-1}$ potassium trifluoroacetate (KTFA) as the eluent at $40{ }^{\circ} \mathrm{C}$ and a flow rate of $0.8 \mathrm{~mL} \cdot \mathrm{min}^{-1}$. The columns were packed with modified silica (PFG column particle size: $7 \mu \mathrm{m}$, porosity: 100 and $1000 \AA$ ). Polymethylmethacrylate (PMMA) standards (Polymer Standards Services GmbH (Mainz, Germany)) were used for calibration and toluene was used as the internal standard. A refractive index detector (G1362A RID) and an UV-VIS detector (at $230 \mathrm{~nm}$ unless otherwise stated; Jasco (Gross-Umstadt, Germany) UV-2075 Plus) were used for polymer detection. MALDI-TOF mass spectra [29] were recorded using a Bruker Reflex II MALDI-TOF mass spectrometer equipped with a $337 \mathrm{~nm} \mathrm{~N}_{2}$ laser. Acceleration of the ions was performed with pulsed ion extraction (PIE, Bruker) at a voltage of $20 \mathrm{kV}$. The analyzer was operated in reflection mode and the ions were detected using a microchannel plate detector. Mass spectra were processed by the X-TOF 5.1.0 software (Bruker (Billerica, MA, USA)). A solvent-free sample preparation was performed using trans-2-[3-(4-tert-Butylphenyl)-2-methyl-2-propenylidene]malononitrile (DCTB) as the matrix and sodium trifluoroacetate as the cationizing salt. Calibration was carried out using a $\mathrm{C}_{60} / \mathrm{C}_{70}$ fullerene mixture. Infrared (IR) spectroscopy was performed on a Jasco FT/IR-4100 with an ATR sampling accessory (MIRacle, Pike Technologies, Madison, WI, USA) and Spectra Manager 2.0 (Jasco, Gross-Umstadt, Germany) was used for integration.

Surface pressure-area ( $\pi$ vs. A) isotherms were obtained using a Nima Langmuir-Blodgett trough (KSV Nima, (Espoo, Finland), Coventry, type 611) secured inside an acrylic glass box (Bayer, Leverkusen, Germany) as a dust shield. The total trough surface area was $200 \mathrm{~mm} \times 100 \mathrm{~mm}$, and the total trough volume was approximately $150 \mathrm{~mL}$. The effective trough area was controlled by two hydrophobic barriers that compressed the spread film symmetrically and bilaterally at a rate of $5 \mathrm{~cm}^{2} / \mathrm{min}$. Millipore water was used as subphase in all trials. For all experiments, the subphase temperature was $25 \pm 0.1{ }^{\circ} \mathrm{C}$ (15-min delay after the water was filled in and the lipid solution was spread). Prior to each trial, the water surface was cleaned by aspirating off any residue, such that the measured surface pressure remained $<0.1 \mathrm{mN} / \mathrm{m}$ over a full compression. The Langmuir-Blodgett (LB) components were cleaned with absolute ethanol and chloroform before each experiment, and the deionized water subphase was replaced after each measurement. Surface pressure measurements were taken from a Wilhelmy plate (perimeter of $20 \mathrm{~mm} \times 10 \mathrm{~mm}$ ) made out of chromatography paper, which was washed several times with absolute chloroform prior to each trial to ensure cleanliness. Dynamic light scattering measurements were performed at $25^{\circ} \mathrm{C}$ using a Malvern (Malvern, UK) Zetasizer NanoZS with a He/Ne laser $(633 \mathrm{~nm})$ at a fixed angle of $173^{\circ}$.

Synthesis of sarcosine $\mathrm{N}$-carboxyanhydride. The synthesis of sarcosine NCA was adapted from literature and modified. A total of $14.92 \mathrm{~g}(167.4 \mathrm{mmol})$ sarcosine, dried under vacuum for $1 \mathrm{~h}$, was weighed into a pre-dried, three-neck, round-bottom flask. A total of $300 \mathrm{~mL}$ of absolute tetrahydrofurane (THF) was added under a steady flow of nitrogen, $16.2 \mathrm{~mL}$ (134 mmol) of diphosgene was added slowly via syringe, and the nitrogen stream was reduced. The colorless suspension was mildly refluxed for $3 \mathrm{~h}$, yielding a clear solution. Afterward, a steady flow of dry nitrogen was led 
through the solution for another $3 \mathrm{~h}$ while the outlet was connected to two gas washing bottles filled with aqueous $\mathrm{NaOH}$ solution to neutralize phosgene. The solvent was evaporated under reduced pressure, yielding a brownish oil as a crude reaction product. The oil was dried under reduced pressure $\left(1 \times 10^{-3}\right.$ mbar for $\left.2 \mathrm{~h}\right)$ to obtain an amorphous solid, free of phosgene and $\mathrm{HCl}$, confirmed by testing against a silver nitrate solution. The crude product was redissolved in $40 \mathrm{~mL}$ of THF and precipitated with $300 \mathrm{~mL}$ of dry $n$-hexane. The solution was cooled to $-18{ }^{\circ} \mathrm{C}$ and stored for $18 \mathrm{~h}$ to complete precipitation. The solid was filtered under dry nitrogen atmosphere and dried in a stream of dry nitrogen for 60-90 min and afterwards under high vacuum for $2 \mathrm{~h}$ in the sublimation apparatus. The crude product was sublimated at $85{ }^{\circ} \mathrm{C}$ and $1 \times 10^{-3}$ mbar. The product was collected from the sublimation apparatus in a glovebox on the same day. The purified product $(110 \mathrm{mmol}, 65 \%$ yield, colorless crystallites; melting point: $102-104{ }^{\circ} \mathrm{C}\left(\right.$ lit: $\left.102-105^{\circ} \mathrm{C}\right)$ ) was stored in a Schlenk tube at $-80^{\circ} \mathrm{C}$ and only handled in a glovebox.

${ }^{1} \mathrm{H}$ NMR $\left(300 \mathrm{MHz}, \mathrm{CDCl}_{3}\right): \delta / \mathrm{ppm}=4.22\left(2 \mathrm{H}, \mathrm{s},-\mathrm{CH}_{2}-\mathrm{CO}-\right), 2.86\left(3 \mathrm{H}, \mathrm{s},-\mathrm{CH}_{3}\right)$.

Synthesis of polysarcosine. Under nitrogen counter flow, Sar-NCA was transferred into a pre-dried Schlenk tube equipped with a stir bar and again dried under high vacuum for $1 \mathrm{~h}$. Then, the NCA was dissolved in dry DMF to yield a solution of $100 \mathrm{mg} / \mathrm{mL}$ with respect to the NCA. $1 / n$ equivalent of either tetradecyl amine or stearyl amine was dissolved in pre-dried THF and added to the NCA solution. The solution was stirred at room temperature and kept at a constant pressure of 1.25 bar of dry nitrogen via the Schlenk line to prevent impurities from entering the reaction vessel while allowing $\mathrm{CO}_{2}$ to escape. Completion of the reaction was confirmed by Fourier transform infrared (FTIR) spectroscopy (disappearance of the NCA peaks $\left(1853\right.$ and $\left.1786 \mathrm{~cm}^{-1}\right)$ ). After completion of the reaction, the polymer was precipitated with cold ether and centrifuged ( $4500 \mathrm{rpm}$ at $4{ }^{\circ} \mathrm{C}$ for $15 \mathrm{~min}$ ). After discarding the liquid fraction, new ether was added and the polymer was resuspended in a sonic bath. The suspension was centrifuged again and the procedure was repeated. After complete DMF removal by the resuspension steps, the polymer was dissolved in water and lyophilized, obtaining a colorless, stiff and porous solid.

${ }^{1} \mathrm{H}$ NMR (400 MHz; DMSO-d $\left.d_{6}\right): \delta / p p m: ~ 4.43-3.83\left(14 \mathrm{H} ; \mathrm{br} ;(2 n)-\mathrm{CO}-\mathrm{CH}_{2}-\mathrm{NH}-\right)$; 3.14-2.65 (23H; br; (3n)-N- $\left.\mathrm{CH}_{3}-\right)$; 1.53-1.12 (32H; br; $\left.-\mathrm{CH}_{2}-\left(\mathrm{CH}_{2}\right)_{16}-\mathrm{CH}_{3}\right) ; 0.86\left(3 \mathrm{H} ; \mathrm{t} ;-\mathrm{CH}_{2}-\mathrm{CH}_{3}\right)$.

Synthesis of carboxy functionalized polymers. The polymer was dissolved in dry DMF with 10 eq. (with respect to the polymer end group) of diisopropylethylamine (DIPEA) and stirred for $30 \mathrm{~min}$. To this solution the 5 eq. succinic acid anhydride was added and stirred overnight at room temperature. The excess of DIPEA and succinic anhydride were removed by dialysis and the product was lyophilized. Complete removal was verified by DOSY ${ }^{1} \mathrm{H}$ NMR.

${ }^{1} \mathrm{H}$ NMR (400 MHz; DMSO- $\left.d_{6}\right)$ : $\delta / \mathrm{ppm}$ : 4.69-3.72 (97H; br; $\left.(2 n)-\mathrm{CO}-\mathrm{CH}_{2}-\mathrm{NH}-\right)$; 3.10-2.66 (152H; br; (3n)-N-CH $\left.{ }_{3}^{-}\right) ; 2.42-2.25$ (4H; br; - $\left.\mathrm{CO}-\left(\mathrm{CH}_{2}\right)_{2}-\mathrm{COOH}\right) ; 1.47-1.17\left(32 \mathrm{H} ; \mathrm{br} ;-\mathrm{CH}_{2}-\left(\mathrm{CH}_{2}\right)_{16}-\mathrm{CH}_{3}\right)$; $0.86\left(3 \mathrm{H} ; \mathrm{t} ;-\mathrm{CH}_{2}-\mathrm{CH}_{3}\right)$.

Synthesis of acetylated polymers. The polymer was dissolved in dry DMF with 10 eq. of DIPEA and stirred for $30 \mathrm{~min}$. To this solution the 5 eq. acetic anhydride or the FITC was added and stirred overnight at room temperature. Excess DIPEA and acetic acid anhydride were removed by dialysis and the product was lyophilized. Complete removal was verified by ${ }^{1} \mathrm{H}-\mathrm{DOSY}$ NMR.

${ }^{1} \mathrm{H}$ NMR (400 MHz; DMSO- $\left.d_{6}\right): \delta / \mathrm{ppm}$ : 4.55-3.77 (99H; br; $\left.(2 n)-\mathrm{CO}-\mathrm{CH}_{2}-\mathrm{NH}-\right)$; 3.22-2.63 $(154 \mathrm{H}$; br; (3n)-N-CH $\left.{ }_{3}^{-}\right)$; $2.06-1.90\left(3 \mathrm{H} ; \mathrm{br} ;-\mathrm{NCH}_{3}-\mathrm{CO}-\mathrm{CH}_{3}\right) ; 1.49-1.14\left(32 \mathrm{H} ; \mathrm{br} ;-\mathrm{CH}_{2}-\left(\mathrm{CH}_{2}\right)_{16}-\mathrm{CH}_{3}\right) ; 0.86$ $\left(3 \mathrm{H} ; \mathrm{t} ;-\mathrm{CH}_{2}-\mathrm{CH}_{3}\right)$.

Synthesis of FITC labeled polymers. The polymer was dissolved in dry DMF with 10 eq. of DIPEA and stirred for $30 \mathrm{~min}$. To this solution 2 eq. FITC was added and stirred overnight at room temperature. Excess DIPEA and FITC were removed by dialysis and the product was lyophilized. Complete removal was verified by ${ }^{1} \mathrm{H}$-DOSY NMR.

${ }^{1} \mathrm{H}$ NMR (400 MHz; DMSO- $\left.d_{6}\right)$ : $\delta /$ ppm: 10.32-9.87 (1H; br; FITC-COOH); 8.37-7.37 (3H; br; aromatic $-\mathrm{CH}-\mathrm{C}-\mathrm{COOH}-$; aromatic $\mathrm{CH}-\mathrm{CH}-\mathrm{COH}$; aromatic $\mathrm{CH}-\mathrm{CH}-\mathrm{CNHR})$; 6.74-6.24 (6H; br; 
-CH-CO-CH-CH-; -COH-CH-COR-; CH-CH-CNHR); 4.81-3.71 (93H; br; (2n)-CO-CH $-\mathrm{CH}_{2}-$ ); 3.14-2.65 (142H; br; (3n)-N-CH $3-)$; $1.54-1.10$ (32H; br; $\left.-\mathrm{CH}_{2}-\left(\mathrm{CH}_{2}\right)_{16}-\mathrm{CH}_{3}\right)$; $0.86\left(3 \mathrm{H} ; \mathrm{t} ;-\mathrm{CH}_{2}-\mathrm{CH}_{3}\right)$.

CMC measurements. CMCs have been determined with a Dataphysics (Filderstadt, Germany) ring tensiometer (DCATIIEC) at $25^{\circ} \mathrm{C}$. It was calibrated against deionized water purified with a Milli-Q system (Merck Millipore, Darmstadt, Germany) to $18.2-\mathrm{M} \Omega \mathrm{cm}$ resistivity and TOC $<5 \mathrm{ppb}$. All samples were aged for 30 min prior to use.

Dynamic light scattering measurements: Lipopeptoids were dissolved in phosphate buffered saline (PBS) to yield $0.1 \mathrm{mg} / \mathrm{mL}$. Prior to measurement samples were aged for $30 \mathrm{~min}$.

Cellular Toxicity: Toxicity studies were carried out using the CellTiter-Glo ${ }^{\circledR}$ Luminescent Cell Viability Assay by Promega. The assay was carried out following the manufacturers' protocol. HeLa cells were cultured in RPMI medium with 10\% heat-inactivated fetal bovine serum (FCS). Cells were harvested at $60 \%-70 \%$ confluence, incubated at $37{ }^{\circ} \mathrm{C}, 95 \%$ relative humidity (rh) and $5 \% \mathrm{CO}_{2}$ for $24 \mathrm{~h}$ in a 24-well plate with lipopeptoids. Lipopeptoids were dissolved in PBS. Medium was replaced $1 \mathrm{~h}$ prior to experiments. The experiments were performed in triplicate. Data was normalized to the untreated control.

Langmuir Blodgett layer formation: Solutions of the polymer-lipid mixtures in chloroform were spread on the subphase by using a microsyringe (Kloehn, Las Vegas, NV, USA). In a typical experiment, 20-30 $\mu \mathrm{L}$ of the solution was spread dropwise onto the water surface so that a constant mass of lipid was deposited for each trial. The spreading solution was deposited at regularly spaced locations on the trough. In all trials, a 15-min evaporation period between the last deposited drop of solution and the beginning of compression was employed to ensure complete solvent evaporation.

\section{Results}

\subsection{Synthesis of Lipopolypeptoids}

The synthesis of $100 \mathrm{mg}$ to $1 \mathrm{~g}$ of lipopolymer was conducted using freshly sublimated Sar-NCA, purified solvent (DMF) and initiator (tetradecyl or stearyl amine). The polymerizations were carried out at room temperature with a monomer concentration of $0.1 \mathrm{~g} / \mathrm{mL}$. After complete monomer conversion was ensured by FTIR-measurements (disappearance of NCA attributed carbonyl vibration band at 1786 and $1850 \mathrm{~cm}^{-1}$ ) lipopeptoids were precipitated in cold diethylether. Afterwards, lipopolymers were dried by lyophilization from water and analyzed by ${ }^{1} \mathrm{H}$ NMR, HFIP SEC, MALDI-TOF, and ${ }^{1} \mathrm{H}-\mathrm{DOSY}$ NMR. ${ }^{1} \mathrm{H}$ NMR experiments displayed that the deviation of the obtained degrees to those calculated are below 10\% (Table 1). In hexafluoroisopropanole (HFIP) SEC the synthesized lipopolymers indicate a symmetric narrowly-distributed molecular weight distribution. The PMMA equivalent molecular weights are in the range of 5 to $25 \mathrm{~kg} / \mathrm{mol}$, while dispersities are between 1.05 and 1.13 (see Table 1). Furthermore, SEC clearly demonstrates that the hydrodynamic volume scales with the degree of polymerization (Figure 1a). An influence of the initiator on the control over polymerization was not detectable, as both aliphatic amines lead to a well-controlled polymerization. To ensure the formation of lipopolymers and the absence of PSar homopolymers ${ }^{1} \mathrm{H}$-DOSY NMR experiments have been carried out. The diffusion ordered NMRs confirm the absence of low molecular weight or high molecular weight side-products, since only a single diffusing polymer species is detected, which contains all PSar, as well as lipid attributed proton signals.

In the next step, the methylamine end groups were quenched with fluoresceine isothiocyanate (FITC), succinic acid anhydride (COOH functionality), or acetic acid anhydride (neutral end group) in the presence of diisopropylethylamine (DIPEA) (Scheme 1). While acetylated PSar could be used as a stealth-only material, carboxylic acid-functionalized lipopolypeptoids are accessible to further modifications since targeting moieties e.g., antibodies or sugars can be attached. Labeling with a dye, in this case FITC, will allow analysis of these formulations with fluorescent techniques, e.g., fluorescent correlation spectroscopy, confocal microscopy, and fluorescence activated cell sorting. To monitor the end group modification efficiency further ${ }^{1} \mathrm{H}$-DOSY NMR experiments have been conducted. This 
method cannot only help to ensure that end group modification is complete, but also confirm the successful removal of the small molecules used for the polymer modification during workup (dialysis). (Appendix A Figure A1) With respect to the limits of ${ }^{1} \mathrm{H}$ NMR spectroscopy, these experiments reveal the absence of impurities as well as the quantitative conversion of PSar end groups.

Table 1. Polymer analysis of amphiphilic PSars.

\begin{tabular}{cccccc}
\hline Polymer & $\boldsymbol{X}_{\mathbf{n}}$ (Calculated) & $\boldsymbol{X}_{\mathbf{n}}$ (NMR) & $\boldsymbol{X}_{\mathbf{n}}$ (MALDI) & $\boldsymbol{M}_{\mathbf{n}}$ (GPC) & $\boldsymbol{\Xi}$ \\
\hline $\mathrm{C}_{18}$ PSar $_{12}$ & 10 & 12 & & 5232 & 1.06 \\
$\mathrm{C}_{18}$ PSar $_{30}$ & 30 & 30 & & 7619 & 1.06 \\
$\mathrm{C}_{18}$ PSar $_{45}$ & 50 & 45 & 47 & 13,933 & 1.07 \\
$\mathrm{C}_{18}$ PSar $_{64}$ & 70 & 64 & & 15,090 & 1.08 \\
$\mathrm{C}_{18}$ PSar $_{117}$ & 100 & 117 & & 24,890 & 1.13 \\
$\mathrm{C}_{14}$ PSar $_{11}$ & 10 & 11 & & 3960 & 1.05 \\
$\mathrm{C}_{14}$ PSar $_{34}$ & 30 & 34 & 49 & 14,718 & 1.07 \\
$\mathrm{C}_{14}$ PSar $_{53}$ & 50 & 53 & & 18,220 & 1.12 \\
$\mathrm{C}_{14}$ PSar $_{75}$ & 70 & 75 & & 23,210 & 1.11 \\
$\mathrm{C}_{14}$ PSar $_{103}$ & 100 & 103 & & & \\
\hline
\end{tabular}

(a)

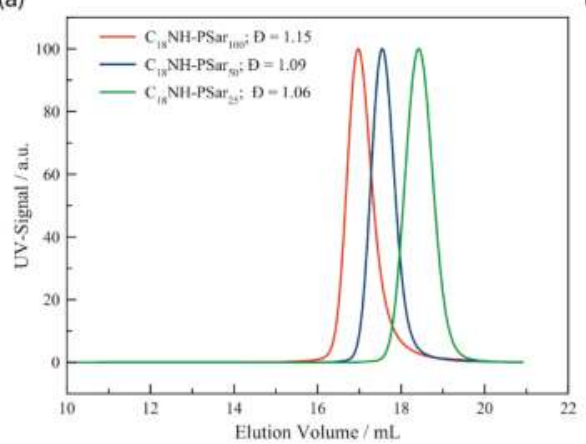

(c)

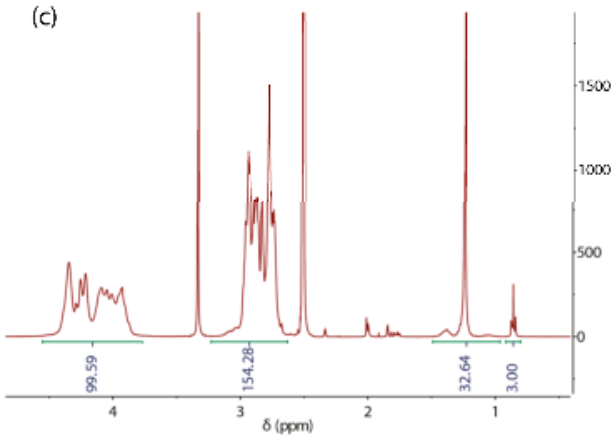

(b)

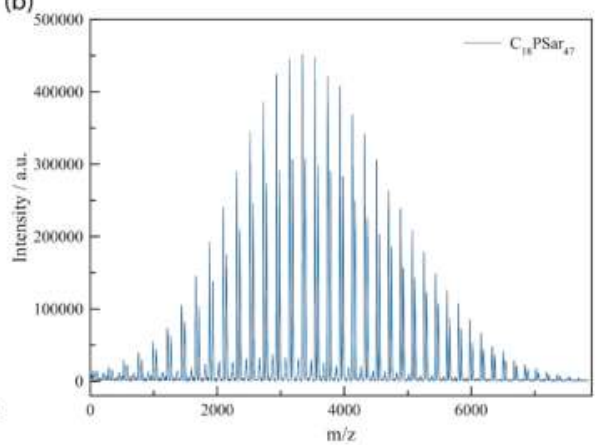

(d)

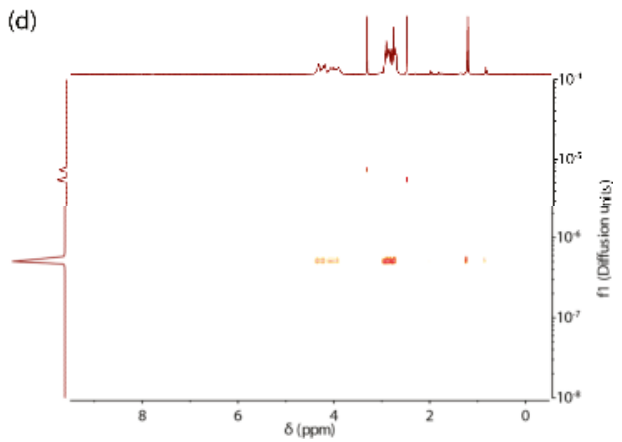

Figure 1. Exemplary polymer analysis of lipopolypeptoids (a) HFIP SEC of $\mathrm{C}_{18}$ PSar $_{34}, \mathrm{C}_{18} \mathrm{PSar}_{45}$, and $\mathrm{C}_{18} \mathrm{PSar}_{117}$; (b) MALDI TOF MS of $\mathrm{C}_{18} \mathrm{PSar}_{47}$; (c) ${ }^{1} \mathrm{H}$ NMR of $\mathrm{C}_{18} \mathrm{PSar}_{47}$; and (d) ${ }^{1} \mathrm{H}$ DOSY NMR of $\mathrm{C}_{18} \mathrm{PSar}_{47}$.

After the synthesis, solution properties of lipopolypeptoids are investigated, which are the critical micelle concentration (CMC) and the hydrodynamic diameter $\left(D_{\mathrm{h}}\right)$ in aqueous solution by dynamic light scattering (DLS). First, we calculated the hydrophilic-lipophilic balance (HLB) for the synthesized lipopolymers. 


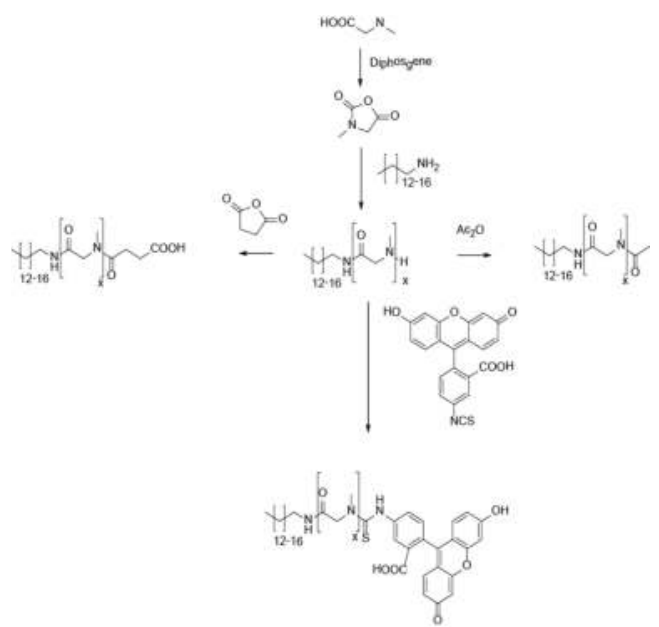

Scheme 1. A synthetic pathway to heterotelechelic lipopolypeptoids.

\subsection{Characterization, Solution Properties, and Cellular Toxicity of Lipopolypeptoids}

According to W.C. Griffin the hydrophilic-lipophilic balance (HLB) is defined as [30]:

$$
\mathrm{HLB}=20 \times\left(1-\frac{M_{\text {lipohilic }}}{M_{\text {lipopeptoid }}}\right)
$$

Therefore, the HLB values are between 15 and 20 for the synthesized lipopolymers, which is a HLB range for solubilizing agents in general (see Table 2). Solubilizing agents are a class of amphiphiles, which are hardly able to incorporate water soluble substances into micellar structures. This process is called micellar solubilization, or, briefly, solubilization [31]. In comparison to other amphiphiles, solubilizing agents have higher HLB values and biocompatible ones are often used to solubilize hydrophobic drugs [32].

Table 2. Calculated HLB values and measured CMC.

\begin{tabular}{ccccc}
\hline Polymer & HLB value $^{\mathbf{1}}$ & $\mathbf{C M C}^{\mathbf{2}} \mathbf{m g} \cdot \mathbf{L}^{-\mathbf{1}}$ & Diameter of main peak (DLS/nm) & Distribution \\
\hline $\mathrm{C}_{18}$ PSar $_{12}$ & 16.0 & 27 & 10.1 & bimodal \\
$\mathrm{C}_{18}$ PSar $_{30}$ & 17.8 & 62 & 13.7 & bimodal \\
$\mathrm{C}_{18}$ PSar $_{45}$ & 18.4 & 94 & 19.1 & monomodal \\
$\mathrm{C}_{18}$ PSar $_{65}$ & 19.0 & 181 & 25.2 & monomodal \\
$\mathrm{C}_{18}$ PSar $_{117}$ & 19.5 & 1181 & 24.9 & monomodal \\
$\mathrm{C}_{14}$ PSar $_{11}$ & 15.7 & 213 & 9.1 & monomodal \\
$\mathrm{C}_{14}$ PSar $_{34}$ & 18.4 & - & 12.7 & bimodal \\
$\mathrm{C}_{14}$ PSar $_{53}$ & 18.9 & - & 16.4 & monomodal \\
$\mathrm{C}_{14}$ PSar $_{75}$ & 19.1 & - & 19.2 & bimodal \\
$\mathrm{C}_{14}$ PSar $_{103}$ & 19.4 & - & 24.4 & trimodal \\
\hline &
\end{tabular}

With respect to their HLB values, all synthesized lipopolymers can be considered as solubilizing agents, which raises the question of PSar chain length dependency of CMCs and hydrodynamic diameter of micelles. Therefore, ring tensiometry was used to determine CMC values of the synthesized lipopolypeptoids. In the applied tensiometer setup the surface tension at about 70 different 
concentrations was measured. With increasing amounts of surfactant the surface tension of the solution decreases. Once the CMC is reached the surface tension remains constant and micelles start to form. All additional surfactants added to the system increase the micellar fraction (Figure 2). Within the series of $\mathrm{C}_{18}$ lipopolypeptoids, the CMC increases with the degree of polymerization of the PSar block and, thus, with HLB values from $0.027\left(\mathrm{C}_{18} \mathrm{PSar}_{12}\right)$ to $1.181 \mathrm{~g} / \mathrm{L}\left(\mathrm{C}_{18} \mathrm{PSar}_{117}\right)$ (See Table 1). As expected, lipopolypeptoids with $\mathrm{C}_{14}$ alkyl chain have significantly higher $\mathrm{CMCs}$ than those with $\mathrm{C}_{18}$ alkyl chain. The aggregation concentration is so high that only for $\mathrm{C}_{14} \mathrm{PSar}_{11}$ a CMC could be determined in the applied concentration range. Thus, CMCs of the other $\mathrm{C}_{14}$ lipopolymers are above $1.500 \mathrm{~g} / \mathrm{L}$.

After determining CMC values, the synthesized lipopolypeptoids micelles have been further investigated. Therefore, a concentration of $1 \mathrm{~g} / \mathrm{L}$ was chosen, which is well above the CMC of the corresponding $\mathrm{C}_{18}$ lipopolymers. Only the lipopolymers with a $\mathrm{C}_{14}$ alkyl chain and the $\mathrm{C}_{18} \mathrm{PSar}_{117}$ have been characterized at a concentration of $10 \mathrm{~g} / \mathrm{L}$. The series of $\mathrm{C}_{18}$ lipopolypeptoids formed aggregates, which increase in size with the degree of polymerization. The aggregates have diameters between $10.1 \mathrm{~nm}\left(\mathrm{C}_{18}\right.$ PSar $\left._{12}\right)$ and $24.9 \mathrm{~nm}\left(\mathrm{C}_{18}\right.$ PSar $\left._{117}\right)$ (see Table 2). For the $\mathrm{C}_{14}$ lipopolypeptoids the trend is also confirmed, since those micelles have diameters between $9.6 \mathrm{~nm}\left(\mathrm{C}_{14} \mathrm{PSar}_{11}\right)$ and $28.1 \mathrm{~nm}\left(\mathrm{C}_{14} \mathrm{PSar}_{103}\right) . \mathrm{C}_{14} \mathrm{PSar}_{103}$ also shows a unimer fraction next to the micellar fractions (Figure 3 ). Micelles formed by $\mathrm{C}_{18}$ and $\mathrm{C}_{14}$ lipopolypeptoids have a comparable diameter at comparable degrees of polymerization. For the PSars with $X_{n}<35$ a fraction of several hundred $\mathrm{nm}$ is observed. The number-weighted distribution does not display these fractions (Figure A3), which is due to the overestimation of large fractions in intensity weighted plotting. Since these polymers have only a single alkyl chain, hydrophobic stabilization of the micelles is very low. This leads to relatively high CMCs and, consequently, to a dynamic system with high exchange rates, which seems to be the reason for the formation of more complex aggregates.

In the next step and with a view using lipopolypeptoids as excipients in drug formulations, the cellular toxicity of lipopolypeptoids was investigated in HeLa cells using the CellTiter-Glo ${ }^{\circledR}$ assay. This assay is a method of determining the number of viable cells based on quantitation of the ATP values present, an indicator of metabolically active cells. The quantification relies on a proprietary thermostable luciferase, which generates a stable "glow-type" luminescent signal depending on ATP levels. In relation to untreated cells as the positive control, relative cell viability can be determined. In this case lipopolypeptoids showed no toxicity up to a concentration of $50 \mu \mathrm{M}$. For a concentration of $500 \mu \mathrm{M}$ (1.2 to $4.2 \mathrm{mg}$ ) the lipopolymers with low degrees of polymerization showed toxicity, which was not observed for lipopolymers with high PSar content (Figure 4).

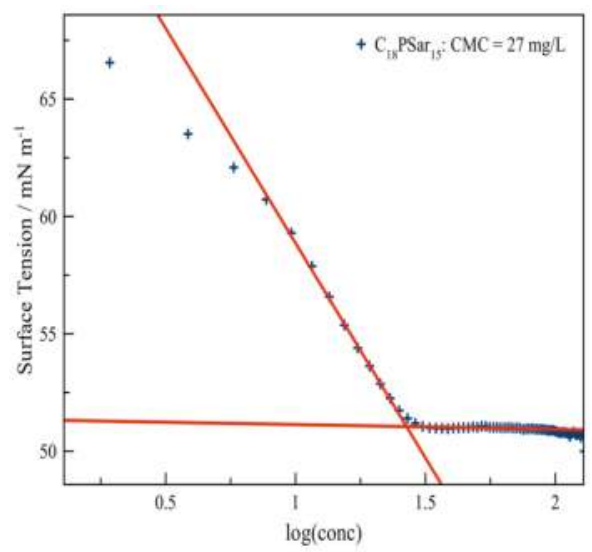

Figure 2. Exemplary $\mathrm{CMC}$ measurement shown for $\mathrm{C}_{18} \mathrm{PSar}_{15}$. $\mathrm{CMC}$ was determined by ring tensiometry. Intersection of the two lines represents the CMC value. 

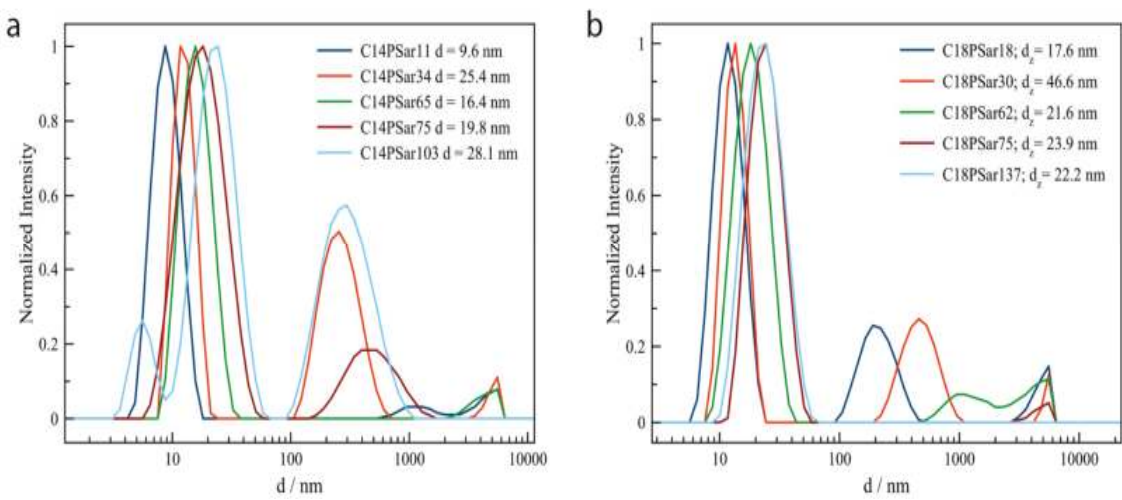

Figure 3. Intensity-weighted diameter distribution of lipopolypeptoids in aqueous solution determined by dynamic light scattering (DLS). (a) Series of tetradecyl amine $\left(\mathrm{C}_{14}\right)$-based lipopolypeptoids; and (b) series of stearyl amine $\left(\mathrm{C}_{18}\right)$-based lipopolypeptoids.

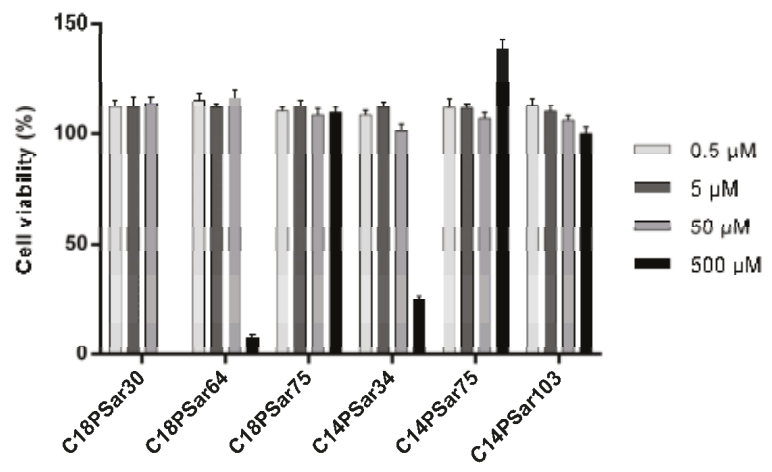

Figure 4. CellTiter-Glo ${ }^{\circledR}$ (CTG) assay toxicity studies of unformulated amphiphilic PSars in HeLa cells in the concentration range from 0.5 up to $500 \mu \mathrm{M}$. Data was normalized to the untreated control. For better comparability molar concentrations were chosen. $1.2 \mathrm{mg} / \mathrm{mL}\left(\mathrm{C}_{18} \mathrm{PSar}_{30}\right)$ and $4.2 \mathrm{mg} / \mathrm{mL}$ $\left(\mathrm{C}_{14} \mathrm{PSar}_{103}\right)$ correspond to $500 \mu \mathrm{mol}$.

\subsection{Formation and Characterization of Lipopolypeptoid Containing Langmuir-Blodgett Monolayers}

In the last part of this study, and with regard to the incorporation of lipopolypeptoids in liposomes to provide stealth-like properties, it was investigated to which extent lipopolypeptoids can be incorporated into lipid membranes of 1,2-distearoyl-sn-glycero-3-phosphocholine (DSPC). On a Langmuir-Blodgett trough solutions of the lipopolymer-lipid mixture in chloroform were spread on the subphase. After evaporation of the chloroform, the area was compressed with a speed of $5 \mathrm{~cm}^{2} / \mathrm{min}$. Plotting the surface pressure over the area, a similar behavior is observed for a polymer content up to $3 \mathrm{~mol} \%$. When the compression starts pure DSPC lipids are in the gas analogous phase. At a pressure from 0 to $3.8 \mathrm{mN} / \mathrm{m}$ lipids are in the liquid analogous phase having an average area of $70 \AA^{2} / \mathrm{mol}$. At $55 \AA^{2} / \mathrm{mol}$ the slope of the curve rises and the solid analogous phase is reached. For the mixture bearing $1 \%$ lipopolypeptoids the phase transitions remain at the same area/molecule, but the transition from liquid to solid analogous phase takes place at $6.7 \mathrm{mN} / \mathrm{m}$. Having a mixture with $2 \%$ lipopolypeptoids the onset point of the liquid analogous phase starts at $92 \AA^{2} / \mathrm{mol}$. The surface pressure rises slowly to $8.15 \mathrm{mN} / \mathrm{m}$ and a small plateau is reached at $59 \AA^{2} / \mathrm{mol}$ (coexistence 
of liquid and solid analogous phase). Transition to solid analogous phase remains at $55 \AA^{2} / \mathrm{mol}$ at a surface pressure of $10.7 \mathrm{mN} / \mathrm{m}$. The phase transition to liquid analogous phase in the mixture with $3 \%$ $\mathrm{C}_{18} \mathrm{PSar}_{47}$ starts at $100 \AA^{2} / \mathrm{mol}$. The coexistence of solid and liquid analogous phase end at a surface pressure of $7.8 \mathrm{mN} / \mathrm{m}$ and $60 \AA^{2} / \mathrm{mol}$.

The area per molecule when the membranes collapse remains constant at around $35 \AA^{2} / \mathrm{mol}$ for $0 \%, 1 \%, 2 \%$, and $3 \%$. The surface pressure at the collapsing point is between 45 and $55 \mathrm{mN} / \mathrm{m}$ and thus comparable to monolayers formed by DSPC alone $(65 \mathrm{mN} / \mathrm{m}$ ) (Figure 5$)$. To prove that no polymer was squeezed out during multiple compression and expension processes, cyclic measurements were carried out up to a surface pressure of $25 \mathrm{mN} / \mathrm{m}$. This pressure was chosen to be well below the corruption point, but being in the solid-analogous phase. The hysteresis plots overlay with each other, indicating that none or only a minor loss of polymer occurs even for the highest lipopolypeptoid content of $3 \mathrm{~mol} \%$ (Figure A2).

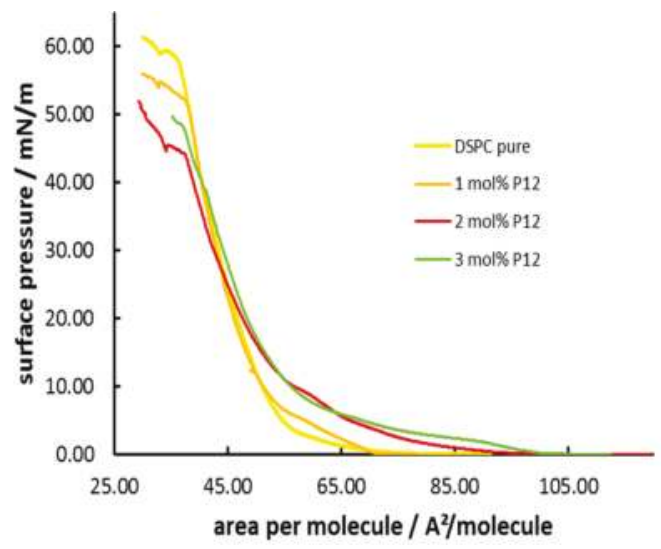

Figure 5. Isothermal surface pressure of a Langmuir-Blodgett monolayer at $25{ }^{\circ} \mathrm{C}$ with different amounts of lipopolypeptoids (pure DSPC, 1\%, 2\%, and 3\% $\mathrm{C}_{18} \mathrm{PSar}_{45}$ ).

\section{Discussion}

In contrast to the early work on lipopolypeptoids published by Gallot and coworkers [28,33] lipopolypeptoids displayed narrow molecular weight distributions and low dispersity indices of approximately 1.1 when synthesized according to the synthetic methods reported in this paper. According to MALDI-TOF MS data there are no detectable side products. Furthermore, we could demonstrate quantitative end group modification using anhydrides or isothiocyanates (ITC) by NMR studies ( ${ }^{1} \mathrm{H}$ NMR and ${ }^{1} \mathrm{H}$-DOSY-NMR). Therefore, we do not see any reason for lipopolymer fractionation as reported by Gallot and coworkers [33]. Eventually the lack of control over the polymerization reported by the authors is related to the suboptimal choice of chloroform as a solvent for the reaction. As already demonstrated DMF [34-36], NMP or benzonitrile [18] should be preferred for the synthesis of such systems, since they ensure dissolution and enable the living ring opening polymerization of Sar-NCAs.

Kolliphor EL, formerly known as Cremophor EL, is a glycerol-based amphiphile, bearing about 35 PEG units in total and three hydrophobic tails. In comparison to the CMCs of Kolliphor EL of $90 \mathrm{mg} / \mathrm{L}$ lipopolypeptoids of comparable HLB values, e.g., $\mathrm{C}_{18} \mathrm{PSar}_{11}$, have a three-fold lower CMC of $27 \mathrm{mg} / \mathrm{L}$ [37]. In comparison to other PEGylated castor oils (e.g., PEG44 CO CMC: $958.2 \mathrm{mg} / \mathrm{mL}$ [38]) polysarcosine based lipopolypeptoids have an even ten-fold lower CMC at comparable HLB values. Since the $\mathrm{A}_{2}$ parameters in water between PEG and PSar are practically identical (unpublished data), this is somehow an unexpected result. But as PSar has an amide in each monomer unit, it has a less 
flexible backbone compared to PEG. This leads to a stretching of the polymer resulting in a closer packing and, therefore, to a higher micelle stability. Furthermore, the hydrophobic tails differ, so that the stacking of the hydrophobic domains also deviates.

These findings relate to the performed DLS measurements. Diameters found for the micelles are in the range of 9-25 nm. Polymers with a higher HLB value and a higher amount of polysarcosine assemble into larger aggregates compared to those with a smaller degree of polymerization. These studies show high dispersities on a micellar level ranging from 0.2-0.4. For $\mathrm{C}_{14} \mathrm{PSar}_{103}(D=0.7)$ unimers and larger aggregates are detected in addition to the micellar fraction. Moreover, intensity weighted DLS displays larger structures of several hundred nanometers for some lipopolypeptoids $\left(\mathrm{C}_{18} \mathrm{PSar}_{12}, \mathrm{C}_{18} \mathrm{PSar}_{30}, \mathrm{C}_{14} \mathrm{PSar}_{34}\right.$, and $\left.\mathrm{C}_{14} \mathrm{PSar}_{75}\right)$. However, these larger aggregates do not appear in the number weighted plot. This underlines that only a very small ratio assembles into large aggregates, being overestimated by intensity-weighted DLS. In comparison with PSar-based block copolymers, the lipopolypeptoid-based micelles are less uniform than those based on polypept(o)ides, [39] while they are comparable with aggregates formed by amphiphilic block copolypeptoids [40]. Likely, dispersities of assemblies can be lowered by methods for more controlled solution self-assembly, extrusion, or other preparation techniques. A formulation of these lipopolypeptoids with, for example, a lipid or hydrophobic molecules will lead to a better hydrophobic stabilization and, therefore, to more uniform structures. These studies, also of great interest, are beyond the scope of the current article.

The reported amphiphiles with a low degree of polymerization, show toxicity at $500 \mu \mathrm{M}$, which corresponds to $1.2 \mathrm{mg} / \mathrm{mL}$. At $50 \mu \mathrm{mol}$ no toxicity was detected in HeLa cells. Other polysarcosinebased materials have been reported to be non-toxic in HeLa cells up to $3 \mathrm{mg} / \mathrm{mL}$ by Birke et al. [34]. A polymeric hydrophobic block stabilizes aggregates more than an alkyl tail. The slower dynamics in these micelles compared to the reported lipopolypeptoids lead to reduced interference with cell membranes. Cremophor EL, as an example for a PEG-based system, shows toxicity at low concentrations $(0.1 \mathrm{~g} / \mathrm{L})$ in endothelial cells [41].

Langmuir monolayers of pure DSPC have a phase transition to the solid analogous phase at $55 \AA^{2} /$ mol. This is the same value as reported by Hao et al. [42] It can also be seen, that with increasing PSar content the isotherms of the lipopolymers are shifted to higher area/molecule and a pseudo-plateau is reached at a surface pressure between 8 and $11 \mathrm{mN} / \mathrm{m}$. PEG-based systems reported by Tanwir also showed pseudo-plateaus with an onset of 8 and $9 \mathrm{mN} / \mathrm{m}$ for $1 \%$ and $3 \%$ PEGylated lipid (degree of polymerization $X_{n}=45$ ) [43]. The reported collapsing pressure of $59 \mathrm{mN} / \mathrm{m}$ is a little higher than for our systems $(45-55 \mathrm{mN} / \mathrm{m})$. Since the degrees of polymerization are comparable, this finding may be attributed to the differences in lipid parts of the lipopolymer. In comparison to the PEGylated DPPE-based system reported by Tanwir the reported lipopolypeptoids bear only a single alkyl chain.

\section{Conclusions}

In this work, we report synthetic pathways to lipopolypeptoids with adjustable HLB values, precise control over molecular weights, dispersity indices, and end group integrity. The synthesized lipopolymers are of amphiphilic nature and self-assemble into micelles or more complex aggregates above their CMC in aqueous solution. The lipopolymers are non-toxic to cells up to a concentration of $50 \mu \mathrm{mol}$, which is a more than 10 times higher value comaqred to Cremophor EL. This finding points to a potential application as excipients in drug formulations. In addition, $\mathrm{C}_{18} \mathrm{PSar}_{45}$-based lipopolymers can be incorporated into Langmuir Blodgett monolayers based on DSPC up to a concentration of $3 \mathrm{~mol} \%$ without altering its properties, which indicates the use of such lipopolypeptoids in the preparation of stealth-like liposomes. Therefore, the reported experiments are a first indication that polysarcosinylated lipids, named lipopolypeptoids, may be applied as bio-based excipients in drug or lipid formulations. 
Acknowledgments: All sources of funding of the study should be disclosed. Please clearly indicate grants that you have received in support of your research work. Clearly state if you received funds for covering the costs to publish in open access.

Author Contributions: Benjamin Weber, Regine Süss and Matthias Barz conceived and designed the experiments; Benjamin Weber, Christine Seidl, David Schwiertz, Stefan Bleher and Martin Scherer performed the experiments; Benjamin Weber, and Matthias Barz analyzed the data; Benjamin Weber and Matthias Barz wrote the paper.

Conflicts of Interest: The authors declare no conflict of interest.

\section{Appendix A}

a

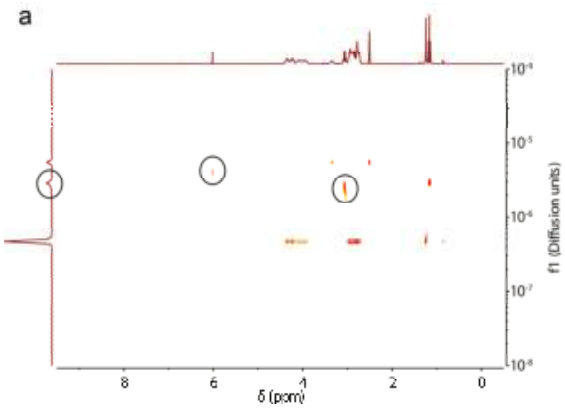

b

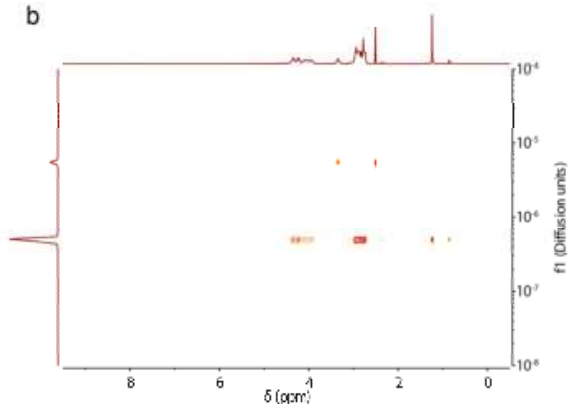

Figure A1. DOSY ${ }^{1} \mathrm{H}$ NMR of $\mathrm{C}_{18} \mathrm{PSar}_{45} \mathrm{COOH}(\mathbf{a})$ before, and (b) after removal of succinic anhydride.

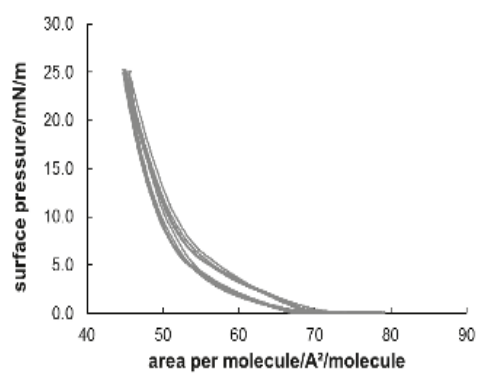

Figure A2. Isothermic compression and expansion hysteresis at $25^{\circ} \mathrm{C}$ with $3 \%$ polymer content.
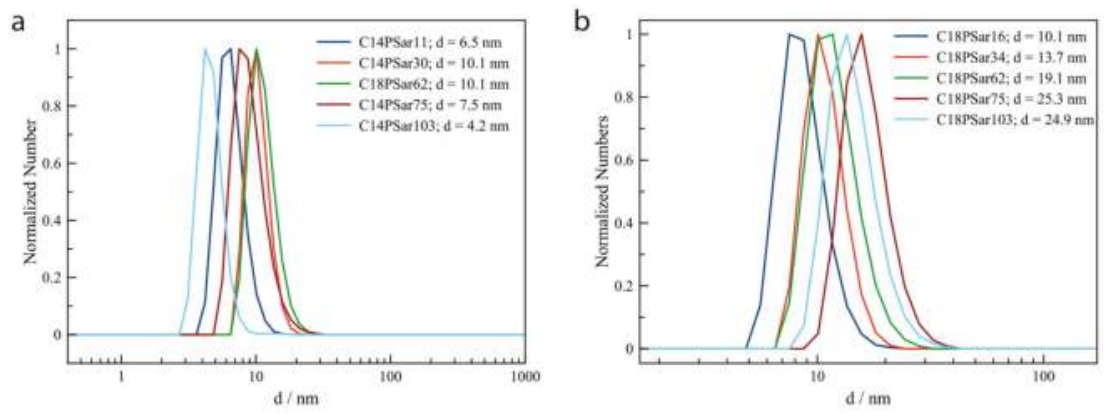

Figure A3. Number weighted diameter distribution of lipopolypeptoid determined by dynamic light scattering (DLS). (a) Series of tetradecyl amine $\left(\mathrm{C}_{14}\right)$ based lipopolypeptoids; and (b) Series of stearyl amine $\left(\mathrm{C}_{18}\right)$ based lipopolypeptoids. 


\section{References}

1. Kosaric, N. Biosurfactants: Production, Properties, Applications; CRC Press: New York, NY, USA, 1993.

2. Holmberg, K. Natural surfactants. Curr. Opin. Colloid Interface Sci. 2001, 6, 148-159. [CrossRef]

3. Raffa, P.; Wever, D.A.Z.; Picchioni, F.; Broekhuis, A.A. Polymeric surfactants: Synthesis, properties, and links to applications. Chem. Rev. 2015, 115, 8504-8563. [CrossRef] [PubMed]

4. Lutz, J.F. Solution self-assembly of tailor-made macromolecular building blocks prepared by controlled radical polymerization techniques. Polym. Int. 2006, 55, 979-993. [CrossRef]

5. Laschewsky, A. Molecular concepts, self-organisation and properties of polysoaps. In Polysoaps/Stabilizers/ Nitrogen-15 NMR; Springer: Berlin/Heidelberg, Germany, 1995; pp. 1-86.

6. Charalambopoulou, A.; Bokias, G.; Staikos, G. Template copolymerisation of $N$-isopropylacrylamide with a cationic monomer: Influence of the template on the solution properties of the product. Polymer 2002, 43, 2637-2643. [CrossRef]

7. Clapés, P.; Infante, M.R. Amino acid-based surfactants: Enzymatic synthesis, properties and potential applications. Biocatal. Biotransform. 2002, 20, 215-233. [CrossRef]

8. Yokota, H.; Sagawa, K.; Eguchi, C.; Takehara, M. New amphoteric surfactants derived from lysine. I. Preparation and properties of $\mathrm{NE}$-acyllysine derivatives-acyllysine derivatives. J. Am. Oil Chem. Soc. 1985, 62, 1716-1719.

9. Baschang, G.; Hartmann, A.; Wacker, O. Lipopeptides Having Antitumor Activity. U.S. Patent 4666886 A, 19 May 1987.

10. Braun, D. Developments with lipoamino acids and their salts. Cosmet. Toilet. 1989, 104, 87-96.

11. Xia, J. Protein-Based Surfactants: Synthesis: Physicochemical Properties, and Applications; CRC Press: Boca Raton, FL, USA, 2001.

12. Saadati, R.; Dadashzadeh, S.; Abbasian, Z.; Soleimanjahi, H. Accelerated blood clearance of PEGylated PLGA nanoparticles following repeated injections: Effects of polymer dose, PEG coating, and encapsulated anticancer drug. Pharm. Res. 2013, 30, 985-995. [CrossRef] [PubMed]

13. Ishida, T.; Harada, M.; Wang, X.Y.; Ichihara, M.; Irimura, K.; Kiwada, H. Accelerated blood clearance of PEGylated liposomes following preceding liposome injection: Effects of lipid dose and PEG surface-density and chain length of the first-dose liposomes. J. Control. Release 2005, 105, 305-317. [CrossRef] [PubMed]

14. Hara, E.; Makino, A.; Kurihara, K.; Yamamoto, F.; Ozeki, E.; Kimura, S. Pharmacokinetic change of nanoparticulate formulation "Lactosome" on multiple administrations. Int. Immunopharmacol. 2012, 14, 261-266. [CrossRef] [PubMed]

15. Ulbricht, J.; Jordan, R.; Luxenhofer, R. On the biodegradability of polyethylene glycol, polypeptoids and poly(2-oxazoline)s. Biomaterials 2014, 35, 4848-4861. [CrossRef] [PubMed]

16. Barz, M.; Luxenhofer, R.; Zentel, R.; Vicent, M.J. Overcoming the PEG-addiction: Well-defined alternatives to PEG, from structure-property relationships to better defined therapeutics. Polym. Chem. 2011, 2, 1900-1918. [CrossRef]

17. Gangloff, N.; Ulbricht, J.; Lorson, T.; Schlaad, H.; Luxenhofer, R. Peptoids and polypeptoids at the frontier of supra- and macromolecular engineering. Chem. Rev. 2015, 116, 1753-1802. [CrossRef] [PubMed]

18. Luxenhofer, R.; Fetsch, C.; Grossmann, A. Polypeptoids: A perfect match for molecular definition and macromolecular engineering? J. Polym. Sci. Part A 2013, 51, 2731-2752. [CrossRef]

19. Sisido, M.; Imanishi, Y.; Higashimura, T. Nuclear magnetic resonance spectra of poly( $N$-alkylamino acid)s. Biopolymers 1972, 11, 399-408. [CrossRef] [PubMed]

20. Klinker, K.; Barz, M. Polypept(o)ides: Hybrid systems based on polypeptides and polypeptoids. Macromol. Rapid Commun. 2015, 36, 1943-1957. [CrossRef] [PubMed]

21. Fetsch, C.; Grossmann, A.; Holz, L.; Nawroth, J.F.; Luxenhofer, R. Polypeptoids from N-substituted glycine $N$-carboxyanhydrides: Hydrophilic, hydrophobic, and amphiphilic polymers with poisson distribution. Macromolecules 2011, 44, 6746-6758. [CrossRef]

22. Gangloff, N.; Fetsch, C.; Luxenhofer, R. Polypeptoids by living ring-opening polymerization of $N$-substituted N-carboxyanhydrides from solid supports. Macromol. Rapid Commun. 2013, 34, 997-1001. [CrossRef] [PubMed]

23. Chapman, R.G.; Ostuni, E.; Liang, M.N.; Meluleni, G.; Kim, E.; Yan, L.; Pier, G.; Warren, H.S.; Whitesides, G.M. Polymeric thin films that resist the adsorption of proteins and the adhesion of bacteria. Langmuir 2001, 17, 1225-1233. [CrossRef] 
24. Lau, K.H.A.; Ren, C.; Park, S.H.; Szleifer, I.; Messersmith, P.B. An experimental-theoretical analysis of protein adsorption on peptidomimetic polymer brushes. Langmuir 2012, 28, 2288-2298. [CrossRef] [PubMed]

25. Secker, C.; Brosnan, S.M.; Luxenhofer, R.; Schlaad, H. Poly( $\alpha$-peptoid)s revisited: Synthesis, properties, and use as biomaterial. Macromol. Biosci. 2015, 15, 881-891. [CrossRef] [PubMed]

26. Kidchob, T.; Kimura, S.; Imanishi, Y. Amphiphilic poly(Ala)-b-poly(Sar) microspheres loaded with hydrophobic drug. J. Control. Release 1998, 51, 241-248. [CrossRef]

27. Yamamoto, F.; Yamahara, R.; Makino, A.; Kurihara, K.; Tsukada, H.; Hara, E.; Hara, I.; Kizaka-Kondoh, S.; Ohkubo, Y.; Ozeki, E.; et al. Radiosynthesis and initial evaluation of (18)F labeled nanocarrier composed of poly(L-lactic acid)-block-poly(sarcosine) amphiphilic polydepsipeptide. Nucl. Med. Biol. 2013, 40, 387-394. [CrossRef] [PubMed]

28. Gallot, B. Liposarcosine-Based Polymerizable and Polymeric Surfactants; American Chemical Society: Washington, DC, USA, 1991; pp. 103-113.

29. Trimpin, S.; Rouhanipour, A.; Az, R.; Räder, H.J.; Müllen, K. New aspects in matrix-assisted laser desorption/ionization time-of-flight mass spectrometry: A universal solvent-free sample preparation. Rapid Commun. Mass Spectrom. 2001, 15, 1364-1373. [CrossRef] [PubMed]

30. Griffin, W.C. Classification of surface-active agents by "HLB". J. Soc. Cosmet. Chem. 1949, 1, 311-326.

31. Naught, A.D.M.; Wilkinson, A. IUPAC Gold Book, 2nd ed.; Blackwell Scientific Publication: Oxford, UK, 1997.

32. Strickley, R.G. Solubilizing excipients in oral and injectable formulations. Pharm. Res. 2004, 21, 201-230. [CrossRef] [PubMed]

33. Douy, A.; Gallot, B. New amphipathic lipopeptides, 1. Synthesis and mesomorphic structures of lipopeptides with polysarcosine peptidic chains. Die Macromol. Chem. 1986, 187, 465-483.

34. Birke, A.; Huesmann, D.; Kelsch, A.; Weilbächer, M.; Xie, J.; Bros, M.; Bopp, T.; Becker, C.; Landfester, K.; Barz, M. Polypeptoid-block-polypeptide copolymers: Synthesis, characterization, and application of amphiphilic block Copolypept(o)ides in drug formulations and miniemulsion techniques. Biomacromolecules 2014, 15, 548-557. [CrossRef] [PubMed]

35. Heller, P.; Weber, B.; Birke, A.; Barz, M. Synthesis and sequential deprotection of triblock copolypept(o)ides using orthogonal protective group chemistry. Macromol. Rapid Commun. 2015, 36, 38-44. [CrossRef] [PubMed]

36. Holm, R.; Klinker, K.; Weber, B.; Barz, M. Synthesis of Amphiphilic Block Copolypept(o)ides by Bifunctional Initiators: Making PeptoMicelles Redox Sensitive. Macromol. Rapid Commun. 2015, 36, 2083-2091. [CrossRef] [PubMed]

37. Kessel, D. Properties of cremophor EL micelles probed by fluorescence. Photochem. Photobiol. 1992, 56, 447-451. [CrossRef] [PubMed]

38. El-Azab, W.I.M.; Aiad, I.; Azzam, E.M.S.; Gad, E.A.M. Molecular, Surface, and thermodynamic properties of nonionic surfactants based on castor oil. J. Dispers. Sci. Technol. 2010, 31, 1150-1156. [CrossRef]

39. Huesmann, D.; Sevenich, A.; Weber, B.; Barz, M. A head-to-head comparison of poly(sarcosine) and poly(ethylene glycol) in peptidic, amphiphilic block copolymers. Polymer 2015, 67, 240-248. [CrossRef]

40. Fetsch, C.; Gaitzsch, J.; Messager, L.; Battaglia, G.; Luxenhofer, R. Self-assembly of amphiphilic block copolypeptoids-Micelles, worms and polymersomes. Sci. Rep. 2016, 6, 33491. [CrossRef] [PubMed]

41. Kiss, L.; Walter, F.R.; Bocsik, A.; Veszelka, S.; Ózsvári, B.; Puskás, L.G.; Szabó-Révész, P.; Deli, M.A. Kinetic analysis of the toxicity of pharmaceutical excipients cremophor EL and RH40 on endothelial and epithelial cells. J. Pharm. Sci. 2013, 102, 1173-1181. [CrossRef] [PubMed]

42. Hao, C.; Liu, Q.; Li, Q.; Zhang, J.; Sun, R. Thermodynamic and structural studies of DMPC and DSPC with DOTAP mixed monolayers at the air-water interface 1. Russ. J. Phys. Chem. A 2016, 90, 214-219. [CrossRef]

43. Tanwir, K.; Tsoukanova, V. Lateral distribution of a poly(ethylene glycol)-grafted phospholipid in phosphocholine monolayers studied by epifluorescence microscopy. Langmuir 2008, 24, 14078-14087. [CrossRef] [PubMed]

(C) 2016 by the authors. Licensee MDPI, Basel, Switzerland. This article is an open access article distributed under the terms and conditions of the Creative Commons Attribution (CC BY) license (http:/ / creativecommons.org/licenses/by/4.0/). 


\title{
Review \\ Dynamics of Polymer Translocation: A Short Review with an Introduction of Weakly-Driven Regime
}

\author{
Takahiro Sakaue ${ }^{1,2}$ \\ 1 Department of Physics, Kyushu University, Fukuoka 819-0395, Japan; sakaue@phys.kyushu-u.ac.jp; \\ Tel.: +81-92-802-4066 \\ 2 Precursory Research for Embryonic Science and Technology (PRESTO), \\ Japan Science and Technology Agency, Honcho Kawaguchi, Saitama 332-0012, Japan \\ Academic Editors: Alexander Böker and Frank Wiesbrock \\ Received: 14 October 2016; Accepted: 1 December 2016; Published: 7 December 2016
}

\begin{abstract}
As emphasized in a recent review (by V.V. Palyulin, T. Ala-Nissila, R. Metzler), theoretical understanding of the unbiased polymer translocation lags behind that of the (strongly) driven translocation. Here, we suggest the introduction of a weakly-driven regime, as described by the linear response theory to the unbiased regime, which is followed by the strongly-driven regime beyond the onset of nonlinear response. This provides a concise crossover scenario, bridging the unbiased to strongly-driven regimes.
\end{abstract}

Keywords: polymer translocation; memory effect; generalized Langevin equation; nonequilibrium dynamic; tension propagation

\section{Introduction}

Macromolecules can be transported through a nanoscale pore by threading it. This process of polymer translocation has been extensively studied for the last two decades [1-8]. The motivation for the research arises from its relevance to biopolymer transport in living cells, and most notably, its connection to the nanopore-based new genome sequencing technique [9].

There are various important factors in the problem, including the electrokinetic effect, the pore properties (size, geometry, and interaction with polymer, etc.), and the capture process of polymer into the pore. Among others, one can ask how to characterize the very process of the polymer going through a simple pore, which is a purely a problem of polymer dynamics. The central quantity here is the translocation time $\tau$ as a function of the chain length $N$ and the driving force $f$. Many efforts have been devoted to clarifying the scaling formula of $\tau$, which is expected to be universal for long enough polymers $[7,8]$.

In the literature, the situation is categorized either by unbiased $(f=0)$ or driven (finite $f$ ) translocation, depending on the absence or the presence of a driving force. In either case, an essential aspect in the problem lies in the collective dynamics of polymer associated with the tension propagation, which is manifested by the anomalous dynamics of the translocation coordinate (see Figure 1). In the present paper, we shall introduce the weakly-driven translocation regime in between. As will be shown, this provides a concise crossover scenario connecting three regimes. In Section 2, we first recall the memory effect approach to the unbiased translocation proposed by Panja, Barkema, and Ball [10]. In Section 3, we then construct the weakly-driven regime through the linear response analysis to the unbiased regime. For larger driving force beyond the onset of nonlinear response $f>k_{B} T /\left(a N^{v}\right)$ ( $a$ : monomer size, $k_{B} T$ : thermal energy), we enter the strongly-driven regime-the basic features of which are, by now, well understood. (For early theoretical and numerical attempts, see References [11-15]. The subsequent revision to the approach in References [11-13] can be found in References [16-22].) We shall briefly review it in Section 4. Summary and discussions are given in Section 5. 


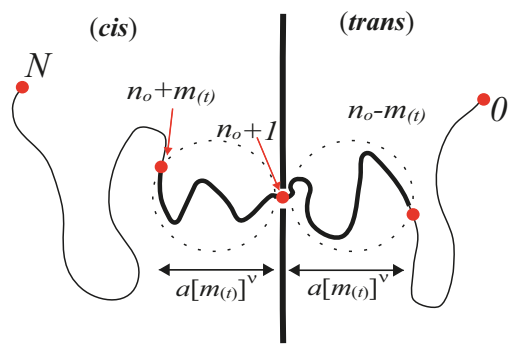

Figure 1. Illustration of a translocating polymer and the transport operation $(\Delta n=1)$ to measure the stress relaxation associated with the tension propagation. The translocation coordinate is defined as the monomer label $n(t)$ at the pore, which counts the number of monomers already in the trans side at time $t$ in analogy to the reaction coordinate in chemical reaction process $[7,8,23,24]$.

\section{Unbiased Translocation}

We first summarize self-similar dynamical properties of a flexible polymer, which will be used in subsequent discussions. Consider a partial section (with $m<N$ bonds) of a long polymer. Its characteristic spatial size is $R_{m} \simeq a m^{v}$, with $v \simeq 3 / 5$ in 3-dimensional space. Associated with it is the characteristic time $\tau_{m} \simeq \tau_{0}\left(R_{m} / a\right)^{z}$, where $\tau_{0}$ is a monomer time scale, and the dynamical exponent is $z=2+v^{-1}$ in the case of free-draining dynamics (see Appendix A.1).

Suggested from these two relations is

$$
m(t) \simeq\left(t / \tau_{0}\right)^{1 /(v z)}
$$

which describes how the tension created by perturbations propagates along the chain. By setting $m\left(\tau_{p}\right) \simeq N$, we find the propagation time

$$
\tau_{p} \simeq \tau_{0} N^{v z}
$$

at which the tension reaches the chain end. This is nothing but the longest time $\tau_{N}$ for the conformational relaxation.

\subsection{Memory Function for Stress Relaxation}

Panja et al. suggested that-due to the monomer exchange across the pore-there exists an imbalance in the tension near the pore, which is responsible for the subdiffusion of the translocation coordinate [10]. The problem can thus be naturally analyzed within the framework of the linear response theory. Consider a polymer going through a narrow pore from the head $(n=0)$. Assume the polymer is in equilibrium with $n_{0}$ bonds in the trans side of the membrane, while the remaining $N-n_{0}$ bonds are on the cis side; i.e., the monomer's label at the pore is $n(t)=n_{0}(t<0)$ (Figure 1). This may be realized by letting the polymer equilibrate with the immobilization constraint imposed on the monomer $n_{0}$ at the pore. Then, at $t=0$, we instantly transport $\Delta n$ monomer from cis to trans. By this operation, the translocation coordinate $n(t)$ is changed from $n_{0}$ to $n_{0}+\Delta n$. The polymer in the trans side is compressed, while it is stretched in the cis side, which produces the restoring force to the translocation coordinate.

To keep the imposed change in the translocation coordinate $n(t)=n_{0}+\Delta n$ at $t>0$, the force is required whose magnitude decreases with time along with the conformational relaxation. This process can be analyzed by the force balance equation

$$
\int_{t_{0}}^{t} d s \Gamma(t-s) v(s)=f(t)
$$


where $v(t)=d n(t) / d t$, and we may set the lower bound of the time integral as $t_{0} \rightarrow-\infty$ by assuming the system is already in the equilibrium state before the operation is made. In the case of step displacement $\Delta n$ imposed at $t=0$ (i.e., $n(t+0)=n(t-0)+\Delta n$ ), we have $v(t)=\Delta n \delta(t)$. The above equation is simplified to

$$
\Delta n \Gamma(t)=f(t)
$$

To evaluate the memory kernel $\Gamma(t)$, it is important to realize that the entire chain cannot respond to the operation at once. At time $t<\tau_{p}$, only a finite section with $m(t)$ bonds given by Equation (1) close to the pore can respond to the operation. The deformation of such a responding chain section on the trans side is evaluated as $\Delta R_{m}=a m(t)^{v}-a\{m(t)+\Delta n\}^{v} \simeq-a\left[m(t)^{v-1} \Delta n\right] v$ (Figure 1). The responding section on the trans side is thus compressed to the amount $\sim a\left[m(t)^{\nu-1} \Delta n\right]$, which exerts a restoring force to the translocation coordinate. The magnitude of the force can be evaluated by noting that the responding domain with $m(t)$ bonds acts as an entropic spring with the spring constant $\simeq k_{B} T / R_{m}(t)^{2}$. We thus find [10]

$$
\begin{aligned}
f(t) & \simeq \frac{k_{B} T}{R_{m}(t)^{2}}\left|\Delta R_{m}\right| \\
& \simeq \frac{k_{B} T}{a m(t)^{1+v}}[v \Delta n] \\
& \simeq \frac{k_{B} T}{a} v \Delta n\left(\frac{t}{\tau_{0}}\right)^{-(1+v) /(v z)}
\end{aligned}
$$

where Equation (1) is used in the last line. For the quantitative discussion, one should note that the same force arises due to the cis side stretching, so the net result doubles.

Comparison of Equations (5) with (4) yields the memory kernel

$$
\Gamma(t) \simeq \frac{k_{B} T}{a} v\left(\frac{t}{\tau_{0}}\right)^{-\alpha}
$$

with the stress relaxation exponent

$$
\alpha=\frac{1+v}{v z}
$$

In general, we have $0<\alpha<1$, reflecting the viscoelastic nature of the response.

\subsection{Unbiased Translocation Dynamics}

To connect the average stress relaxation with the subdiffusion of the translocation coordinate, we need to look at each realization of the stochastic processes. One is then led to the generalized Langevin equation by adding the thermal noise term $\xi(t)$ to the right-hand side of Equation (3), which is zero mean $\langle\mathcal{\zeta}(t)\rangle=0$, and related to the memory kernel via the fluctuation-dissipation theorem $\langle\xi(t) \xi(s)\rangle=k_{B} T \Gamma(|t-s|)$. The equivalent expression of the generalized Langevin equation is

$$
v(t)=\int_{t_{0}}^{t} d s \mu(t-s) f(s)+\eta(t)
$$

with the mobility kernel $\mu(t) \sim-t^{\alpha-2}$ and the noise $\eta(t)$, which again is related to the kernel as $\langle\eta(t) \eta(s)\rangle=k_{B} T \mu(|t-s|)$. In the unbiased case $f(t)=0$, the mean-square displacement (MSD) can be derived after integration of the velocity correlation function $\langle v(t) v(s)\rangle=\langle\eta(t) \eta(s)\rangle$ twice with 
respect to time, yielding $\left\langle\delta n(t)^{2}\right\rangle \sim t^{\alpha}$, where $\delta n(t)=n(t)-n(0)$; i.e., the stress relaxation exponent is equal to the MSD exponent. Therefore, the MSD in translocation coordinate space is obtained as

$$
\left\langle\delta n(t)^{2}\right\rangle \simeq\left(t / \tau_{0}\right)^{(1+v) /(v z)}
$$

\subsection{Traveled Fraction at $t=\tau_{p}$}

The memory kernels obtained for the translocating polymer arise from the viscoelastic response of the polymer due to the tension propagation. Therefore, the memory persists up to the propagation time $\tau_{p}$ given in Equation (2). To find how $\tau_{p}$ is related to the average time $\tau$ for the translocation process, let us calculate the characteristic displacement of the translocation coordinate at the time scale $\tau_{p}$. We find from Equation (9)

$$
\left\langle\delta n\left(\tau_{p}\right)^{2}\right\rangle \simeq N^{1+v} \ll N^{2}
$$

Now let us define the quantity

$$
Q_{N} \equiv \frac{\sqrt{\left\langle\delta n\left(\tau_{p}\right)^{2}\right\rangle}}{N}
$$

which measures a fraction of the total chain length, which is traveled by the time $t=\tau_{p}$. Using Equation (10), we find

$$
Q_{N} \sim N^{-(1-v) / 2} \ll 1
$$

is vanishingly small for asymptotically large $N$. This indicates that the majority of the monomers do not pass the pore by $t=\tau_{p}$; i.e., $\tau \gg \tau_{p}$, so there should be another process to characterize the translocation dynamics. This requires us to think about the post-propagation stage at $t>\tau_{p}$.

\subsection{Post-Propagation Stage}

At $t>\tau_{p}$, the tension has already been propagated up to the chain end, so the motion of all the chain sections is coherent to generate the ordinary diffusion of the translocation coordinate. Here, the relevant question is what is the diffusion coefficient $D_{n}$ for it?

The following matching argument at $t=\tau_{p}$

$$
\left\langle\delta n\left(\tau_{p}\right)^{2}\right\rangle \simeq D_{n} \tau_{p}
$$

where the left-hand side is evaluated by Equation (9), suggests

$$
D_{n} \simeq \tau_{0}^{-1} N^{1+v-v z}
$$

The characteristic time $\tau_{p p}$ for the post-propagation stage is thus obtained as

$$
\tau_{p p} \simeq\left[N\left(1-Q_{N}\right)\right]^{2} / D_{n} \simeq N^{1+(z-1) v}
$$

where the last near-equality is based on the estimation $Q_{N} \ll 1$. Since $\tau_{p p} / \tau_{p} \gg 1$, the scaling formula for the translocation time in the long chain limit becomes $\tau=\tau_{p}+\tau_{p p} \sim \tau_{p p}$ [10].

\section{Weakly-Driven Dynamics}

The process may be biased by the external force. For translocating polymers, the force is acting at the pore, which is realized, for instance, by the voltage drop across the pore. As long as the force is weak enough $f<k_{B} T / R_{N}$, the tension dynamics summarized in the beginning of Section 2 is intact, and the generic linear response argument yields the average dynamics of the translocation coordinate. 
Suppose the system is already in equilibrium, and we start to apply a constant external force $f$ at $t=0$. Equation (8) then simplifies to

$$
\frac{d\langle n(t)\rangle}{d t}=f \int_{0}^{t} d s \mu(s) \sim f t^{\alpha-1}
$$

where, as written after Equation (8), $\mu(t) \sim-t^{\alpha-2}$, with $0<\alpha<1$. The average drift is thus evaluated as

$$
\langle\delta n(t)\rangle \sim f t^{\alpha}
$$

\subsection{Weakly-Driven Translocation Dynamics}

From Equations (7) and (17), the average drift is

$$
\langle\delta n(t)\rangle \sim f t^{(1+v) /(v z)}
$$

Therefore, the drift distance at the propagation time

$$
\left\langle\delta n\left(\tau_{p}\right)\right\rangle \sim N^{1+v} f
$$

We thus find the fraction of the system explored by the propagation time

$$
Q_{N}^{(f)} \equiv \frac{\left\langle\delta n\left(\tau_{p}\right)\right\rangle}{N} \sim N^{v} f<1
$$

where the last inequality comes from $f<k_{B} T / R_{N} \sim N^{-v}$.

\subsection{Post-Propagation Stage}

The result $Q_{N}^{(f)}<1$ for a weakly-driven translocating polymer indicates that the translocation coordinate can explore only a fraction of the system by the propagation time. This conclusion is intact even with the fluctuation effect superimposed, since $n(t)-\langle n(t)\rangle$ is (according to Equation (8)) described by the same dynamical equation as the unbiased dynamics, for which $Q_{N}<1$ as well. Therefore, the post-propagation stage at $t>\tau_{p}$ may become an essential part to determine the whole translocation time $\tau$. Since the tension has already reached the chain ends at $t>\tau_{p}$, we expect the normal drift

$$
\langle\delta n(t)\rangle \simeq \frac{f}{\gamma_{n}} t \quad\left(t>\tau_{p}\right)
$$

where the friction coefficient

$$
\gamma_{n} \sim N^{z v-1-v}
$$

can be determined by matching Equations (19) and (21) at $t=\tau_{p}$ (note the consistency of Equations (22) with (14) through Einstein relation). The characteristic time $\tau_{p p}$ for the post-propagation stage is thus found from the relation $\left(f / \gamma_{n}\right) \tau_{p p}=N-\left\langle\delta n\left(\tau_{p}\right)\right\rangle$; therefore,

$$
\tau_{p p} \simeq \frac{\gamma_{n}}{f} N\left(1-Q_{N}^{(f)}\right) \sim \frac{N^{(z-1) v}}{f}
$$

One can check the dominance of the post-propagation stage from the ratio $\tau_{p p} / \tau_{p}>1$ in the linear response regime. Comparing the two time scales $\tau_{p p}$ in unbiased and weakly-driven regimes, respectively, given by Equations (15) and (23), we find a characteristic force $f \sim N^{-1}$, above which there exists a scaling regime of weakly-driven translocation where $\tau \simeq \tau_{p p}$ given by Equation (23) 
applies. For weaker force $f<1 / N$, the fluctuation effect dominates over the average drift, so we expect a crossover to unbiased scaling, where $\tau \simeq \tau_{p p}$ given by Equation (15) holds.

\section{Strongly-Driven Dynamics}

As pointed out in Reference [11,12] and verified in subsequent works $[14,15,17]$, the nonequilibrium dynamical effect becomes relevant for translocating polymer, which is driven by strong force. Suppose we grab one end of a polymer (with $N$ monomers), and start to pull it by a constant force $f$. When the force is weak enough $f<k_{B} T / R_{N}$, the whole polymer will follow the force without noticeable conformational distortion. For a larger force, however, the polymer will be elongated along the direction of the pulling [25]. The first question here is the relation among the force, the moving velocity, and the elongation in steady state. Such a relation - which we may call a dynamical equation of state-is discussed in detail in Reference [26]. The second question is how we can characterize the transient process toward such an elongated steady state from an initial quiescent state. As one can infer from the scaling form of the threshold force $\sim k_{B} T / R_{N} \sim N^{-v}$, such a nonequilibrium effect shows up with rather moderate force; the "strongly-driven" appellation is only adopted to contrast to the weakly-driven regime discussed in Section 3.

Given a long relaxation time of the whole polymer $\tau_{N} \simeq \tau_{0}\left(R_{n} / a\right)^{z} \simeq \tau_{0} N^{v z}$, it would be intuitively clear that the polymer as a whole cannot respond to the pulling force all at once. Instead, only the subchain part close to the pulled site can initially respond, and thus collectively move in the pulled direction. Such a responding moving domain will grow with time, the dynamics of which is associated with how the tensile force propagates along the chain backbone $[11,12,26]$ (see also [27]). To describe this sort of nonequilibrium response, it is useful to picture the whole polymer as composed of two distinct domains; a moving and a quiescent domain - the latter of which is yet unaware of the pulling force at a given moment. In this two-phase picture, we are interested in the dynamics of the tension front (i.e., domain boundary), which dictates the essential physics in the driven translocation.

Here we follow the argument in Reference [20] (see Appendix A.2). Now, in contrast to the weakly-driven regime (Figure 1), There appears significant dynamical asymmetry between cis and trans sides [28]. To set the stage, let us look at Figure 2. There is a thin wall at $x=0$ with a small pore, where the driving force with the constant magnitude $f>k_{B} T / R_{N}$ is locally exerted in the $x$-direction from the cis to the trans side. One chain end is initially sucked at time $t=0$. The translocation process, then, proceeds with the tension propagation along the chain. Monomers are numbered from the first sucked end to the other end (Nth monomer). The moving domain at time $t$ is specified by the monomer $m(t)$ at the end of the moving domain, the monomer $n(t)$ at the pore, the size $R(t)$, and the representative (or average) velocity $V(t)$. These can be determined by the following set of equations:

$$
\begin{aligned}
V(t) R(t) & \simeq f^{z-2} \\
m(t)-n(t) & \simeq R f^{-(1-v) / v}\left(\simeq R \sigma_{0}\right) \\
m(t)^{v} & \simeq R(t)
\end{aligned}
$$

As explained in Reference [13,26], Equations (24) and (25) are the dynamical equations of state describing the steady-state relation among velocity-extension-force (Equation (24)) and mass-extension-force (Equation (25)) for a dragged polymer. The information on the initial coiled conformation prior to the translocation process is contained in Equation (26). Note that $\sigma_{0} \simeq g_{f} / \xi_{f} \simeq$ $f^{-(1-v) / v}$, where $\xi_{f} \simeq a g_{f}^{v} \simeq k_{B} T / f$ is the size of tensed blob in the close proximity of the pore, is the monomer line density at the pore, so that the monomer flux at the pore is

$$
\frac{d n(t)}{d t}=j_{0}(t)=\sigma_{0} v_{0}(t)
$$

where $v_{0}(t)$ is the velocity of the monomer at the pore site. 

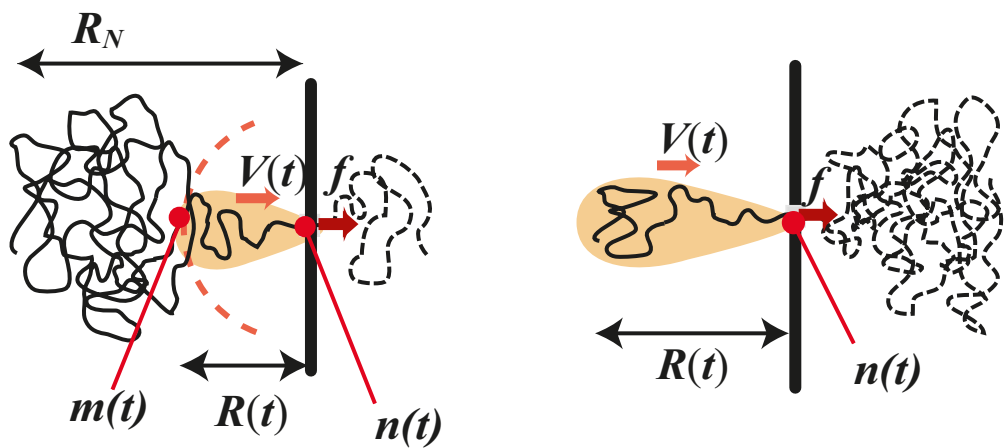

Figure 2. Sketch of a translocating polymer driven by a strong force $f$. (left) Propagation stage: a growing moving domain (with velocity $V(t)$ and the size $R(t)$ ) on the cis side is shaded, while the chain portion already on the trans side is represented by a dashed curve. (right) Post-propagation stage: the tension has already reached to far end of the polymer; thus, $m(t)=N$ is constant, and the moving domain is shrinking with time. In addition, most of monomers are already on the trans side, so this post-propagation stage adds a finite-size correction to the scaling formula of the translocation time.

To solve the above set of equations, we adopt the ansatz $v_{0}(t)=V(t)$ as in Reference [16,18], which amounts to correspond to the iso-flux condition [16,20]. Then, combining Equations (25) and (27), we obtain

$$
\begin{aligned}
& \sigma_{0}\left(V(t)+\frac{d R(t)}{d t}\right) \simeq \frac{d m(t)}{d t} \\
\Leftrightarrow \quad & f^{(z-2)-[(1-v) / v]} R(t)^{-1} \simeq R(t)^{(1-v) / v} \frac{d R(t)}{d t}-f^{-(1-v) / v} \frac{d R(t)}{d t}
\end{aligned}
$$

where Equations (24) and (26), and the expression for the line density at the pore (see Equation (25)) are used. This leads to the tension propagation law

$$
R(t) \sim\left[t f^{(z-2)-[(1-v) / v]}\right]^{v /(1+v)}
$$

which would be valid asymptotically under the condition $f R(t) / k_{B} T \gg 1$. The scaling formula for propagation time is thus obtained by setting $R\left(\tau_{p}^{(f)}\right)=R_{N}$;

$$
\tau_{p}^{(f)} \sim N_{0}^{1+v} f^{-(z-2)+[(1-v) / v]}
$$

Note that the above propagation time can be written as

$$
\tau_{p}^{(f)} \sim \tau_{N}\left(\frac{f R_{N}}{k_{B} T}\right)^{1+(1 / v)-z}<\tau_{N}
$$

where the last inequality - valid in the present condition $f>k_{B} T / R_{N}$-represents the fact that the tension propagation takes place in a time scale shorter than the conformational relaxation time, a clear indication of the nonequilibrium nature of the strongly-driven translocation process. Compare this result with the propagation time Equation (2) for equilibrium (unbiased and weakly-driven) regime.

After $t=\tau_{p}^{(f)}$, the post-propagation stage follows, which can be analyzed by Equation (28), but with $d m(t) / d t=0$ on the right-hand side [13]. A qualitative difference in the dynamical feature 
between propagation and post-propagation stages can be found in the growth rate of $n(t)$; while the slowing-down in the propagation stage is a consequence of the growth of the moving domain, the speed-up in the post-propagation stage is due to the decrease of the overall friction with the process advanced. In contrast to the weakly-driven regime (Section 3), it can be shown that most of the monomers (in a scaling sense) are transported to the trans side in the propagation stage. Therefore, the scaling formula for the translocation time is given by the propagation time (i.e., $\left.\tau \simeq \tau_{p}^{(f)}\right)[11,12,16,19]$ (Note, however, that this does not hold for a semiflexible filament [29]).

To elucidate the crossover between weak and strongly-driven regimes, let us introduce the characteristic size of a tensed blob $\xi_{f} \simeq k_{B} T / f$ and the corresponding time scale $\tau_{f 0} \simeq \tau_{0}\left(\xi_{f} / a\right)^{z}$. In the length scale smaller than $\xi_{f}$, the force is considered to be a weak perturbation. Therefore, under the force with the strength $k_{B} T / R_{N}<f<k_{B} T / a$, the physics in the weakly-driven regime applies up to $t=\tau_{f 0}$, where the tension propagates according to Equation (1). The nonequilibrium effect and the cis-trans dynamical asymmetry manifests in larger length and time scales. In this sense, the length $\xi_{f}$ and time $\tau_{f 0}$ play a role of "initial conditions" for the subsequent nonequilibrium dynamics. In this way, one can show that at $f>k_{B} T / R_{N}$, the tension propagation stage dominates the whole translocation process asymptotically; hence, a natural crossover occurs between two regimes at that force scale.

\section{Summary and Discussions}

\subsection{Summary of the Scaling Formulae}

As stated in Introduction, one of the main purposes of the present note is, aside from reviewing recent progress in the field, to introduce a weakly-driven regime as the linear response domain to the unbiased regime. To show that this provides a concise crossover scenario bridging the unbiased to the strongly-driven regime, let us summarize the scaling formulae for the translocation dynamics in different regimes, according to the classification scheme proposed here.

\subsubsection{Unbiased and Weakly-Driven Regimes}

These two regimes may be termed collectively as the equilibrium regime. The statistical dynamics of the translocation coordinate-characterized here by its first and second moments of the displacement-follows

$$
\begin{aligned}
& \langle\delta n(t)\rangle \sim \begin{cases}f t^{(1+v) /(v z)} & \left(t<\tau_{p}\right) \\
\gamma_{n}^{-1} f t & \left(\tau_{p}<t<\tau\right)\end{cases} \\
& \left\langle\{\delta n(t)-\langle\delta n(t)\rangle\}^{2}\right\rangle \sim \begin{cases}t^{(1+v) /(v z)} & \left(t<\tau_{p}\right) \\
D_{n} t & \left(\tau_{p}<t<\tau\right)\end{cases}
\end{aligned}
$$

where $\gamma_{n} \sim N^{v z-1-v}$ and $D_{n} \sim N^{1+v-v z}$ are the effective friction and diffusion coefficients of the translocation coordinate in the post-propagation stage. For unbiased case $(f=0)$, the drift vanishes, so Equation (34) reduces to the MSD.

The translocation time is dominated by the post-propagation stage $\tau \sim \tau_{p p}$, with

$$
\tau_{p p} \sim \begin{cases}N^{1+(z-1) v} & \left(f<N^{-1}\right) \\ N^{(z-1) v} / f & \left(N^{-1}<f<N^{-v}\right)\end{cases}
$$

Comparing the respective characteristic times of post-propagation stages, the characteristic force $f \simeq k_{B} T /(\mathrm{Na})$ is found; for weaker force, the bias is so weak that the process is essentially unbiased. Note that the same formula for $\tau$ was proposed in Equation (4) of Reference [12], based on the "equilibrium shape assumption". 


\subsubsection{Strongly-Driven Regime}

As described in Section 4, for stronger force $f>k_{B} T / R_{N}$, one has to take the nonequilibrium conformational deformation dynamics into account [11,12]. In such a situation, the response becomes generally nonlinear, and the dynamical asymmetry between cis and trans sides appears [28]. This qualitatively changes the dynamics of tension propagation, hence the structure of the memory effect for the evolution of the translocation coordinate [11-22]. After the initial tensed blob of size $\xi_{f} \simeq k_{B} T / f$ in the vicinity of pore is formed (i.e., $t>\tau_{f 0}$ ), its average time evolution is predicted to follow (see Appendix A.3)

$$
\langle n(t)\rangle \sim \begin{cases}t^{1 /(1+v)} f^{\left(z-1-v^{-1}\right) /(1+v)} & \left(N^{-v}<f<N^{0}\right) \\ t^{1 /(1+v)} f^{1 /(1+v)} & \left(N^{0}<f\right)\end{cases}
$$

where the characteristic force $f \simeq k_{B} T / a$ separates the so-called trumpet and stem-flower regimes [25]. This process persists up to the propagation time $\tau_{p}^{(f)}$ given by $\left\langle m\left(\tau_{p}^{(f)}\right)\right\rangle \sim\left\langle n\left(\tau_{p}^{(f)}\right)\right\rangle \sim N$, which now depends on $f$;

$$
\tau_{p}^{(f)} \sim \begin{cases}N^{1+v} f^{1+(1 / v)-z} & \left(N^{-v}<f<N^{0}\right) \\ N^{1+v} f^{-1} & \left(N^{0}<f\right)\end{cases}
$$

The post-propagation stage follows at $t>\tau_{p}^{(f)}$ [13]. However, in contrast to the unbiased and the weakly-driven regimes, the propagation stage dominates over the post-propagation stage in the sense that the asymptotic translocation time is predicted to be $\tau \sim \tau_{p}^{(f)}[16,19]$. Note that-despite the conspicuous difference in their underlying physics—scaling formulae Equations (35) and (37) are identical in the case of free-draining dynamics $z=2+v^{-1}$. If not, they differ in their scaling structures, but one can check a smooth crossover at $f \sim N^{-v}$. These results on the scaling formulae of the translocation time are summarized in Figure 3.
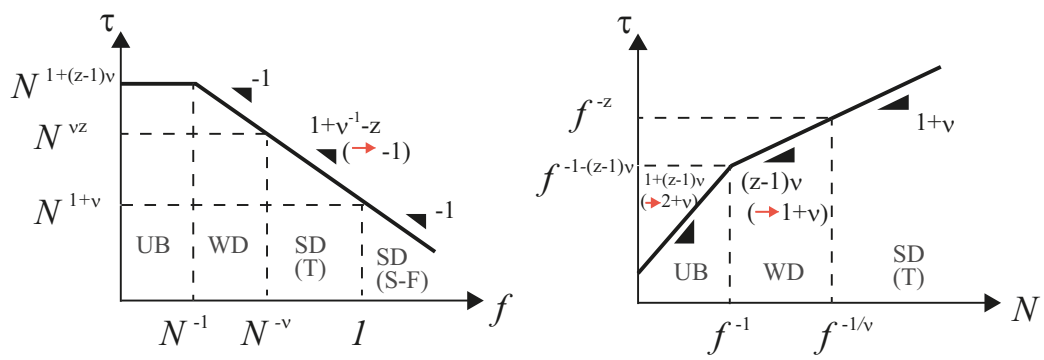

Figure 3. Dependence of the translocation time on $f$ (left) and $N$ (right) shown in double logarithmic scale. The locations of various regimes, unbiased (UB), weakly-driven (WD) and strongly-driven (SD) are specified; the SD regime is further divided into the trumpet (T) and the stem-flower (S-F) regimes. Note that in the right graph, depicted is the case with $f<k_{B} T / a$; otherwise, we have only S-F regime with the slope $1+v$. Note also that in these plots, we set $z=2+v^{-1}$ (free draining dynamics), in which case the plots become particularly simple. The triangles and their nearby numbers designate slopes (exponents), where the numbers after the arrows specify the values for free draining dynamics. For other choices of the dynamical exponent (i.e., $z=3$ for nondraining (Zimm) dynamics), the slope in the SD (T) regime in the left graph is changed. The same applies to UB and WD regimes in the right graph. Comparing these plots with Figure 4 in Reference [16], one finds differences in weak force and short chain length regions. 


\subsection{Discussion}

Following the memory effect approach suggested by Panja et al. [10], we have argued $Q_{N}<1$ for the translocation dynamics in unbiased and weakly driven regimes; thus, $\tau \sim \tau_{p p}$ scales differently from $\tau_{p}$. If, on the contrary, one assumes $\tau \sim \tau_{p}$ as in Chuang, Kantor, and Kardar [30], one ends up with the different scaling for the anomalous diffusion of the translocation coordinate. At present, there seems to be no definite conclusion on which argument is more appropriate, but let us note the following points on this issue from the literature.

In their Langevin dynamics simulation, de Haan and Slater have demonstrated the increasing impact of the memory effect on the translocation dynamics with the increase in the solution viscosity and the chain length $[31,32]$. In such a memory-predominant situation, they have shown that (i) the post-propagation stage dominates the translocation time, and (ii) the translocation time scaling is close to $\tau \sim N^{2+v}$, which is Equation (35) with $z=2+v^{-1}$, in accordance with Panja et al. (see Appendix A.4). However, several reports on the numerically-estimated subdiffusion exponent in the propagation stage do not match the value in Equation (34) [31-33]. The reported exponent looks to be closer to the value suggested by Chuang et al. [30].

It should be kept in mind that we have only considered the asymptotic scaling, which would be valid in the long chain limit. For real situations, a significant finite-size effect would come into play. For strongly-driven translocation, the role of pore friction has been recently elucidated [8,17]. Similar effects would be likely for unbiased and weakly-driven translocations as well, and it might be a possible source for the puzzling observation mentioned above.

In Reference [34], the scaling for the anomalous drift

$$
\langle n(t)\rangle \sim(f t)^{(1+v) /(1+2 v)}
$$

has been proposed based on the combination of memory effect argument similar to ours and the numerical simulation results. However, this is different from our linear response prediction Equation (18), though the exponent on $t$ is the same (note $z=2+v^{-1}$ for the free draining dynamics, so the time exponent is $(1+v) /(v z)=(1+v) /(1+2 v))$. Because of this, a scaling formula for $\tau$ different from Equation (35) was proposed in Reference [34], but this does not seem to provide a clear crossover scenario both to unbiased and strongly-driven regimes.

In conclusion, we have suggested the introduction of the weakly-driven regime for polymer translocation dynamics, which is naturally described by the linear response theory applied to the unbiased regime. A similar discussion on weakly and strongly-driven dynamics for a polymer pulled by mechanical force has been recently done in Reference [35]. We hope the resultant concise crossover scenario will be useful to promote the understanding of polymer translocation dynamics in near-equilibrium situation, which is necessary to unveil a full picture of the phenomenon.

Acknowledgments: I thank D. Panja for discussion and correspondence (which we did in the year 2013), and T. Saito for fruitful collaboration. This work is supported by KAKENHI (No. 16H00804, "Fluctuation and Structure") from MEXT, Japan, and JST, PREST.

Conflicts of Interest: The author declares no conflict of interest.

\section{Appendix A.}

\section{Appendix A.1.}

These two critical exponents are useful for the development of a general scenario. The static exponent $v$ (often called the Flory exponent in polymer literature)-being the inverse of the fractal dimension—quantifies the polymer's spatial extension as a function of its molecular weight. While $v=1 / 2$ for ideal chains, the swelling due to the excluded-volume effect results in a larger value, which depends on the space dimension. On the other hand, the dynamical exponent $z$ is associated with the dissipation mechanism at work. In the simplest model (as adopted in many 
of simulation studies), the motion of monomers caused by the flow of the surrounding medium is neglected. This free-draining dynamics (sometimes called local friction or Rouse dynamics) amounts to set $z=2+v^{-1}$. In dilute solution, however, the solvent-mediated hydrodynamic interaction is known to be relevant. The resultant non-draining dynamics can be approximated by setting $z=3$ (Zimm dynamics), at least, in free space.

Appendix A.2.

This preprint [20] was prepared for a supplemental to Reference [19]. The latter is an erratum to Reference [13], where we have modified earlier scaling predictions [11-13] to be consistent with the appropriate mass conservation relation across the pore, as pointed out in Reference [16].

Appendix A.3.

This relation is deduced from Equation (30) with the relation $m(t) \sim n(t)$, which is expected to be valid asymptotically; see Reference [20].

Appendix A.4.

Notice, however, that the scaling $\tau \sim N^{2}$ was observed in Reference [31,32] in the opposite limit of low viscosity, where the polymer conformational relaxation is rapid. The same scaling is also observed in the limit of high imposed pore friction [36]; see also earlier works [23,24]. For practical purposes, one may add the solvent viscosity and the pore friction as extra parameters (possibly their combination with the chain length) to determine the appropriate regime.

\section{References}

1. Meller, A. Dynamics of polynucleotide transport through nanometre-scale pores. J. Phys. Condens. Matter 2003, 15, R581-R607.

2. Dekker, C. Solid-state nanopores. Nat. Nanotechnol. 2007, 2, 209.

3. Keyser, U.F. Controlling molecular transport through nanopores. J. R. Soc. Interface 2011, 8, 1369-1378.

4. Aksimentiev, A. Deciphering ionic current signatures of DNA transport through a nanopore. Nanoscale 2011, 2, 468-483.

5. Wanunu, M. Nanopores: A journey towards DNA sequencing. Phys. Life Rev. 2012, 9, 125-158.

6. Muthukumar, M. Polymer Translocation; CRC Press: Boca Raton, FL, USA, 2011.

7. Panja, D.; Barkema, G.T.; Kolomeisky, A.B. Through the Eye of the Needle: Recent Advances in Understanding Biopolymer Translocation. J. Phys. Condens. Matter 2013, 25, 413101.

8. Palyulin, V.V.; Ala-Nissila, T.; Metzler, R. Polymer translocation: the first two decades and the recent diversification. Soft Matter 2014, 10, 9016-9037.

9. Branton, D.; Deamer, D.W.; Marziali, A.; Bayley, H.; Benner, S.A.; Butler, T.; Di Ventra, M.; Garaj, S.; Hibbs, A.; Huang, X.; et al. The potential and challenges of nanopore sequencing. Nat. Biotechnol. 2008, 26, 1146-1153.

10. Panja, D.; Barkema, G.T.; Ball, R.C. Anomalous dynamics of unbiased polymer translocation through a narrow pore. J. Phys. Condens. Matter 2007, 19, 432202.

11. Sakaue, T. Nonequilibrium dynamics of polymer translocation and straightening. Phys. Rev. E 2007, $76,021803$.

12. Sakaue, T. Sucking genes into pores: Insight into driven translocation. Phys. Rev. E 2010, 81, 041808.

13. Saito, T.; Sakaue, T. Dynamical diagram and scaling in polymer driven translocation. Eur. Phys. J. E 2011, $34,135$.

14. Lehtola, V.V.; Linna, R.P.; Kaski, K. Dynamics of forced biopolymer translocation. Europhys. Lett. 2009, 85,58006 .

15. Bhattacharya, A.; Binder, K. Out-of-equilibrium characteristics of a forced translocating chain through a nanopore. Phys. Rev. E, 2010, 81, 041804.

16. Rowghanian, P.; Grosberg, A.Y. Force-Driven Polymer Translocation through a Nanopore: An Old Problem Revisited. J. Phys. Chem. B 2011, 115, 14127.

17. Ikonen, T.; Bhattacharya, A.; Ala-Nissila, T.; Sung, W. Influence of non-universal effects on dynamical scaling in driven polymer translocation. J. Chem. Phys. 2012, 137, 085101. 
18. Dubbeldam, J.L.A.; Rostiashvili, V.G.; Milchev, A.; Vilgis, T.A. Forced translocation of a polymer: Dynamical scaling versus molecular dynamics simulation. Phys. Rev. E 2012, 85, 041801.

19. Saito, T.; Sakaue, T. Erratum to: Dynamical diagram and scaling in polymer driven translocation. Eur. Phys. J. E 2012, 35, 125.

20. Saito, T.; Sakaue, T. Two phase picture in driven polymer translocation. arXiv 2012, arXiv:1205.3861v3.

21. Saito, T.; Sakaue, T. Process time distribution of driven polymer transport. Phys. Rev. E 2012, 85, 061803.

22. Sarabadani, J.; Ikonen, T.; Ala-Nissila, T. Iso-flux tension propagation theory of driven polymer translocation: The role of initial configurations. J. Chem. Phys. 2014, 141, 214907.

23. Sung, W.; Park, P.J. Polymer Translocation through a Pore in a Membrane. Phys. Rev. Lett. 1996, 77, 783.

24. Muthukumar, M. Polymer translocation through a hole. J. Chem. Phys. 1999, 111, 10371.

25. Brochard-Wyart, F. Polymer Chains Under Strong Flows: Stems and Flowers. Europhys. Lett. 1995, $30,387$.

26. Sakaue, T.; Saito, T.; Wada, H. Dragging a polymer in a viscous fluid: Steady state and transient. Phys. Rev. E 2012, 86, 011804

27. Grosberg, A.Y.; Nechaev, S.; Tamm, M.; Vasiyev, O. How long does it take to pull an ideal polymer into a small hole? Phys. Rev. Lett. 2006, 96, 228105.

28. Saito, T.; Sakaue, T. Cis-trans dynamical asymmetry in driven polymer translocation. Phys. Rev. E 2013, 88,042606 .

29. Adhikari, R.; Bhattacharya, A. Driven translocation of a semi-flexible chain through a nanopore: A Brownian dynamics simulation study in two dimensions. J. Chem. Phys. 2013, 138, 204909.

30. Chuang, J.; Kantor, Y.; Kardar, M. Anomalous dynamics of translocation. Phys. Rev. E 2001, 65, 011802.

31. De Haan, H.W.; Slater, G.W. Memory effects during the unbiased translocation of a polymer through a nanopore. J. Chem. Phys. 2012, 136, 154903.

32. De Haan, H.W.; Slater, G.W. Using an incremental mean first passage approach to explore the viscosity dependent dynamics of the unbiased translocation of a polymer through a nanopore. J. Chem. Phys. 2012, 136, 204902.

33. Luo, K.; Ollila, S.T.T.; Huopaniemi, I.; Ala-Nissila, T.; Pomorski, P.; Karttunen, M.; Ying, S.-C.; Bhattacharya, A. Dynamical scaling exponents for polymer translocation through a nanopore. Phys. Rev. E 2008, 78, 050901.

34. Vocks, H.; Panja, D.; Barkema, G.T.; Ball, R.C. Pore-blockade times for field-driven polymer translocation. J. Phys. Condens. Matter 2008, 20, 095224.

35. Saito, T.; Sakaue, T. Driven anomalous diffusion: An example from polymer stretching. Phys. Rev. E 2015, 92, 012601.

36. Polson, M.J.; Dunn, T.R. Evaluating the applicability of the Fokker-Planck equation in polymer translocation: A Brownian dynamics study. J. Chem. Phys. 2014, 140, 184904.

(C) 2016 by the author. Licensee MDPI, Basel, Switzerland. This article is an open access article distributed under the terms and conditions of the Creative Commons Attribution (CC BY) license (http:/ / creativecommons.org/licenses/by/4.0/). 
Article

\title{
Investigating the Synergistic Effects of Combined Modified Alginates on Macrophage Phenotype
}

\author{
Hannah C. Bygd ${ }^{1}$ and Kaitlin M. Bratlie ${ }^{1,2,3, *}$ \\ 1 Department of Materials Science \& Engineering, Iowa State University, Ames, IA 50011, USA; \\ hcbygd@iastate.edu \\ 2 Department of Chemical \& Biological Engineering, Iowa State University, Ames, IA 50011, USA \\ 3 Division of Materials Science \& Engineering, Ames National Laboratory, Ames, IA 50011, USA \\ * Correspondence: kbratlie@iastate.edu; Tel.: +1-515-294-7304; Fax: +1-515-294-5444
}

Academic Editor: Alexander Böker

Received: 30 September 2016; Accepted: 1 December 2016; Published: 6 December 2016

\begin{abstract}
Understanding macrophage responses to biomaterials is crucial to the success of implanted medical devices, tissue engineering scaffolds, and drug delivery vehicles. Cellular responses to materials may depend synergistically on multiple surface chemistries, due to the polyvalent nature of cell-ligand interactions. Previous work in our lab found that different surface functionalities of chemically modified alginate could sway macrophage phenotype toward either the pro-inflammatory or pro-angiogenic phenotype. Using these findings, this research aims to understand the relationship between combined material surface chemistries and macrophage phenotype. Tumor necrosis factor- $\alpha$ (TNF- $\alpha$ ) secretion, nitrite production, and arginase activity were measured and used to determine the ability of the materials to alter macrophage phenotype. Cooperative relationships between pairwise modifications of alginate were determined by calculating synergy values for the aforementioned molecules. Several materials appeared to improve M1 to M2 macrophage reprogramming capabilities, giving valuable insight into the complexity of surface chemistries needed for optimal incorporation and survival of implanted biomaterials.
\end{abstract}

Keywords: alginate; macrophage phenotype; TNF- $\alpha$; synergy

\section{Introduction}

Macrophages are functionally diverse cells with a multitude of roles in immunity [1], disease [2], and wound healing [1,3-5], making them an appealing target in research areas like drug delivery [6,7], regenerative medicine [5], and biomaterial implantation [8]. In particular, they are crucial in the initiation, propagation, and resolution stages of the foreign body response (FBR) to implanted biomaterials [9-11]. Macrophages are important in all stages of this response due, in part, to their plasticity and heterogeneous phenotypes. The phenotypes in which macrophages exist are best described as a complex scale, bookended by classical and alternative activations.

Classically activated, M1, macrophages are said to be pro-inflammatory and cytotoxic $[12,13]$. As cells that mediate immune responses to bacterial, viral, and fungal infections, they can be activated by microbial stimuli (lipopolysaccharides (LPS)) or interferon (IFN)- $\gamma$, which is released by activated lymphocytes [14-16]. The inflammatory response that these stimulating factors initiate is followed by the release of important cytotoxic molecules such as reactive nitrogen intermediates (RNIs) and tumor necrosis factor (TNF)- $\alpha$ that can have an impact on the presence of tumor cells $[8,13,16]$. The functions of M1 macrophages also make them important in the inflammation stages of wound healing and the FBR, where they produce pro-inflammatory cytokines, phagocytose microorganisms near the injury, and recruit additional inflammatory cells to the site $[8,10,17-19]$. Alternatively activated, M2, macrophages are known to be pro-angiogenic and can be activated by interleukin (IL)- 4 or IL-13 [20]. 
The main functions of these cells include: maintaining homeostasis, tissue repair and remodeling, as well as promoting wound healing in the resolution stage of the FBR [8]. They fulfill these roles with the release of IL-10, vascular endothelial growth factor (VEGF), and transforming growth factor (TGF)- $\beta$ [21]. The entire spectrum of phenotypes that exists is imperative in one way or another $[4,8]$, but an increased presence of either M1 or M2 macrophages may be useful for specific applications. For example, an earlier M2 macrophage presence rather than prolonged M1 presence may allow for proper wound closure, but in cancer therapies an M1 macrophage presence rather than pro-angiogenic, tumor associated M2 like macrophage, may lead to the elimination of cancerous cells.

With the application of wound healing and successful implant incorporation, the significance of macrophage plasticity is made evident by examining the timeline of macrophage presence during the FBR [8]. Macrophage phenotype is dynamic throughout the entirety of the FBR, and a balance of phenotypes is essential for timely progression from injury to proper healing [8]. After implantation of a biomaterial, macrophages are classically activated by proteins that have adsorbed to the material surface [22-24]. These macrophages release pro-inflammatory cytokines that continue to attract monocytes to the injury site leading to chronic inflammation [19]. Attempting and failing phagocytosis of the large implant, causes the M1 macrophages to fuse into foreign body giant cells (FBGC) [25]. Chronic inflammation is only resolved with a presence of alternatively activated macrophages resulting from phagocytosis of dying cells around the implant, or stimulation by IL-4 or IL-13, typically from basophils [20,26,27]. M2 macrophages seek to promote wound healing in the area of the implant [11]. However, prolonged M2 macrophage activity can lead to the formation of an extensive fibrous capsule that may negatively impact the function of the implant [28]. Finding a balance between chronic inflammation and continued wound healing remains an issue with many biomedical implants. Using the idea that material surface chemistries have an impact on macrophage phenotype, it may be hypothesized that a particular material could orchestrate appropriate progression through the FBR to avoid both chronic wound healing and complete fibrous encapsulation. This may be particularly applicable for electrospinning coaxial fibers [29] and sensors [30], in addition to scaffolds for tissue engineering applications [31].

Many techniques for macrophage reprogramming have been developed that focus on either the chemical or environmental stimuli to which macrophages are exposed [14,15,32,33]. Previous work in this lab has indicated that some chemically modified alginates may impact macrophage phenotype [9]. For example, some modifications decreased TNF- $\alpha$ production by M1 macrophages, which could make them useful for decreasing the inflammatory response to a material. Other modifications appeared to amplify the presence of M2 macrophages by showing an increased arginase/iNOS (inducible nitric oxide synthase) ratio compared to the control. Some of the most promising materials showed decreased TNF- $\alpha$ production and increased arginase/iNOS ratios for all activations of macrophages. Finally, some indicated that even a small amount of modification may be giving the materials some permselectivity capabilities, which could be important for applications in drug delivery and tissue engineering.

Given the complexity of biological systems and the immune system in particular, the idea that the most effective material surface might be made up of several chemistries is reasonable [34]. However, this can make optimal biomaterial design criteria difficult to ascertain [34]. Cellular responses to materials are typically directed by surface protein adsorption [22], and many biological interactions, such as protein-ligand or protein-cell interactions, are polyvalent in nature [22,35]. This suggests a need for a material with multiple surface chemistries to more closely imitate natural interactions, as multiple simultaneous interactions may have unique collective properties that vary from those displayed by each component individually [35]. The synergistic effects of material properties have typically been studied using high-throughput combinatorial approaches [36]. This logic and testing method have similarly been employed in synergistic drug delivery, where the aim is to achieve some synergistic therapeutic effect, reduction of dose or toxicity, and to delay or minimize drug resistance [37-40]. 
The focus of this work was to examine the synergistic effects of a few promising material surface chemistries described above. In order to more accurately describe the phenotype of macrophages in vitro, a newer nomenclature will be used throughout this paper that is based on the molecule used to activate the cells, for example M(LPS) and M(IL-4) [12]. By modifying alginate with one functional group that amplified M(IL-4) activation (ester or oxime) and one that indicated M(LPS) to $\mathrm{M}(\mathrm{IL}-4)$ macrophage reprogramming (amide or nitro), the goal was to achieve an even greater M(IL-4) response from all macrophage phenotypes [9]. This could be useful in the proper integration of various biomaterials. Other materials examined here combine a surface modifier that had high permeability but low production of toxic cytokines (sulfonic acid or nitro) with one that had low permeability but high cytokine production (ketal or epoxide) [9]. An ideal combination of modifications would have low permeability and also cause low production of toxic cytokines. Understanding the synergistic effects of these materials may lead to the development of a diverse library of materials that could influence any desired macrophage phenotype.

\section{Materials and Methods}

Experiments were performed with a minimum of three replicates. Results were compared to controls of unmodified alginate as well as singly modified samples in previous work [9]. All materials were purchased from Sigma (St. Louis, MO, USA) and used as received, unless otherwise indicated. Fresh deionized (DI) water (Milli-Q Nanopure, Thermo Scientific, Waltham, MA, USA) was used throughout this study.

\subsection{Materials}

Surface modifiers [9], coupled to medium viscosity ( 600-900 cps, $\sim 250,000 \mathrm{M}_{\mathrm{W}}$ ) alginate (MP Biomedicals, Santa Ana, CA, USA) were chosen based on the results of previous research in this lab [9]. This included: glycidamide; tert-butyl 4 aminobutanoate (VWR, Radnor, PA, USA); malonamide (Fisher, Pittsburgh, PA, USA); 1-amino-4-oxocyclohexane carboxylic acid ethylene ketal; 2,4-dinitro-phenyl-hydoxylamine; 3-aminobenzamide oxime; and 3-amino-1-propane sulfonic acid (Fisher, Pittsburgh, PA, USA).

\subsection{Alginate Modification}

The seven different surface modifiers were combined and coupled to the medium viscosity alginate using 1-ethyl-3-(3-dimethylaminopropyl)carbodiimide (EDC, Oakwood Chemical, West Colombia, SC, USA) and N-hydroxysuccinimide (NHS, Thermo Scientific, Waltham, MA, USA). These materials were modified as previously described [9] with 50 molar equivalents of modifier 1 and 50 molar equivalents of modifier 2, rather than 100 molar equivalents of one modifier.

\subsection{Elemental Analysis}

In order to determine the percent modification of each material, elemental analysis was performed to obtain $\% \mathrm{C}, \% \mathrm{H}$ and $\% \mathrm{~N}$. Measurements were recorded in triplicate with an acetanilide calibration standard, combustion and reduction temperatures of 925 and $640{ }^{\circ} \mathrm{C}$, respectively, and a resulting accuracy of $\pm 0.3 \%$ for each element. All standards and reagents are from Perkin Elmer and/or Elementar America's Inc. (Mt. Laurel, NJ, USA). The instrument used was a PE 2100 Series II combustion analyzer (Perkin Elmer Inc., Waltham, MA, USA).

\subsection{Water Contact Angle (WCA)}

Samples were prepared by creating a positively charged surface on glass microscope slides with poly-L-lysine (PLL). This allowed for a thin, even coating of each modified alginate to be applied to slides by crosslinking with $\mathrm{SrCl}_{2}$ (Alfa Aesar, Haverhill, MA, USA). These coated slides could then be inverted over a container of water. An air bubble $(100 \mu \mathrm{L})$ was deposited under the slide and 
imaged using a digital camera (Canon EOS Rebel T3i, Canon, Melville, NY, USA). Five replicates were collected for each sample before the angle between the slide and the bubble was measured using Image (NIH, Bethesda, MD, USA) software. The final reported WCA is $180^{\circ}$ minus the measured angle.

\subsection{Compression Modulus}

The compression modulus was measured for each sample using manual compression testing techniques. Modified alginate pegs were made to be approximately $10 \times 10 \times 4 \mathrm{~mm}^{3}$ in size. These pegs were placed between two microscope slides and imaged after each of various sized weights were set on the top slide. The changing distance between the slides in each image was measured using ImageJ, and used to generate stress-strain curves. Linear portions of these curves were used to determine the reported compression modulus for each sample $(n=5)$.

\subsection{Cell Culture}

RAW 264.7 cells (American Type Cell Collection, ATCC, Manassas, VA, USA) were used as a model cell line for macrophages in these experiments. Cells were cultured in complete medium (CM, Dulbecco's modified Eagle's medium (DMEM, Mediatech, Inc., Manassas, VA, USA)) supplemented with 10\% fetal bovine serum (FBS, Mediatech, Inc., Manassas, VA, USA), $100 \mathrm{U} / \mathrm{mL}$ penicillin, and $100 \mu \mathrm{g} / \mathrm{mL}$ streptomycin) at $37{ }^{\circ} \mathrm{C}$ in $5 \% \mathrm{CO}_{2}$. Every three to five days, the cells were passaged using a cell scraper to detach cells and subcultured between $\sim 6.7 \times 10^{3}$ and $2.7 \times 10^{4} \mathrm{cells} / \mathrm{cm}^{2}$.

\subsection{Cell Viability}

In order to test the viability of RAW 264.7 cells in contact with the combined modified alginates, plates were seeded for MTT (methyl thiazolyldiphenyl tetrazolium, Research Products International Corp., Mt Prospect, IL, USA) assays during passaging. For this, 24-well plates (KSE Scientific, Durham, $\mathrm{NC}$, USA) were first coated with $0.05 \%$ PLL solutions using $200 \mu \mathrm{L} /$ well and then incubated at $37^{\circ} \mathrm{C}$ for $1 \mathrm{~h}$. Before seeding the cells into the plate, each well was washed twice with sterile phosphate buffered saline (PBS). The plates were seeded with 125,000 cells $/ \mathrm{cm}^{2}$ in $500 \mu \mathrm{L}$ of CM per well and $25 \mathrm{ng} / \mathrm{mL}$ IL-4 (M(IL-4)) (eBioscience Inc., San Diego, CA, USA) or $5 \mathrm{ug} / \mathrm{mL}$ LPS (M(LPS)) and the cells were allowed to adhere for $24 \mathrm{~h}$. A control set of experiments using non-activated cells was included and referred to as naïve or $\mathrm{M}(0)$ cells. Alginate coatings were created by adding $100 \mu \mathrm{L}$ of modified alginate to each well, allowing it to coat the bottom, and excess alginate was removed. Next, $500 \mu \mathrm{L}$ of $0.2 \mathrm{M} \mathrm{SrCl}_{2}$ were used per well to crosslink the coating. This solution was left in the wells for approximately $5 \mathrm{~min}$ before replacing it with clear CM. After $48 \mathrm{~h}$ the supernatant was removed and saved for further testing. To each well $500 \mu \mathrm{L}$ of clear CM was added along with $50 \mu \mathrm{L}$ of MTT $\left(5 \mathrm{mg} / \mathrm{mL}\right.$ in DI water). After a $2 \mathrm{~h}$ incubation at $37^{\circ} \mathrm{C}, 425 \mu \mathrm{L}$ of the solution was removed from each well and replaced with $500 \mu \mathrm{L}$ of dimethyl sulfoxide (DMSO). The absorbance of each plate was read at $540 \mathrm{~nm}$ with a reference of $690 \mathrm{~nm}$ using a BioTek Synergy HT Multidetection Microplate Reader (BioTek, Winooski, VT, USA). Positive controls for each plate were cells with no alginate samples, and negative controls contained media, modified alginates, and activators. Experiments were performed in quadruplicate and results are given as the mean value for each sample normalized to the positive control \pm standard deviation.

\subsection{TNF- $\alpha$ ELISA}

Measurement of TNF- $\alpha$, an M1 phenotype marker, was performed using commercially available enzyme linked immunosorbent assay (ELISA) kits (eBioscience, Inc., San Diego, CA, USA) and performed as described by the manufacturer, using the supernatant collected during viability assays.

\subsection{Urea Assay}

After $48 \mathrm{~h}$ of incubation with the combined modified alginates, the supernatant was removed and saved for further testing. Cells were then washed with $400 \mu \mathrm{L}$ of PBS, and the plates were 
placed on ice for 10 min with $100 \mu \mathrm{L}$ of cell lysis buffer $(150 \mu \mathrm{L}$ protease inhibitor cocktail (Amresco, Solon, OH, USA) and $15 \mu \mathrm{L}$ Triton X-100 (Acros Technologies, Elgin, IL, USA) diluted to $15 \mathrm{~mL}$ with DI water) per well. The resulting lysate $(25 \mu \mathrm{L})$ was transferred from each well to a 96-well plate (Argos Technologies, Elgin, IL, USA) along with $25 \mu \mathrm{L}$ of a $10 \mathrm{mM} \mathrm{MnCl}_{2}$ (Fisher, Pittsburgh, PA, USA) and $50 \mathrm{mM}$ Tris solution (Fisher, Pittsburgh, PA, USA). The plate was incubated for $10 \mathrm{~min}$ at $55^{\circ} \mathrm{C}$ before adding $50 \mu \mathrm{L}$ of $1 \mathrm{M}$ arginine ( $\mathrm{pH}$ 9.7) to each well and incubating at $37^{\circ} \mathrm{C}$ for $20 \mathrm{~h}$. Arginase activity, an M2 phenotype indicator, was measured through the conversion of arginine to urea. This was done by adding $200 \mu \mathrm{L}$ of a 1:2 ratio of solution 1 (1.2 $\mathrm{g} o$-phthaldialdehyde (Alfa Aesar, Haverhill, MA, USA), $1 \mathrm{~L} \mathrm{H}_{2} \mathrm{O}$, and $500 \mu \mathrm{L} \mathrm{HCl}$ (Fisher, Pittsburgh, PA, USA)) and solution 2 (0.6 g $\mathrm{N}$-(naphthyl)ethylenediamine dihydrochloride (Acros Technologies, Elgin, IL, USA), $5 \mathrm{~g}$ boric acid (Fisher, Pittsburgh, PA, USA), $800 \mathrm{~mL} \mathrm{H}_{2} \mathrm{O}, 111 \mathrm{~mL}$ sulfuric acid (Fisher, Pittsburgh, PA, USA), diluted to $1 \mathrm{~L}$ with $\mathrm{H}_{2} \mathrm{O}$ ) [41]. The plate was read at $520 \mathrm{~nm}$ with a reference at $630 \mathrm{~nm}$.

\subsection{Griess Reagent Assay}

Nitrite production, which is also indicative of an M1 phenotype, was measured from the supernatant collected in the urea assay described above. A standard curve was created using serial dilutions of $100 \mu \mathrm{M} \mathrm{NaNO}_{2}$ with volumes of $150 \mu \mathrm{L}$. To the remaining wells $150 \mu \mathrm{L}$ of sample were added. To the entire plate, $130 \mu \mathrm{L}$ of DI water and $20 \mu \mathrm{L}$ of Griess reagent (Acros Technologies, Elgin, IL, USA) were added and allowed to incubated for $20 \mathrm{~min}$. The plate was read at $448 \mathrm{~nm}$ with a $690 \mathrm{~nm}$ reference.

\subsection{Immunocytochemistry}

RAW 264.7 cells were fluorescently labeled using a previously developed protocol [9]. Briefly, cells were seeded at 100,000 cells $/ \mathrm{cm}^{2}$ on clean, PLL coated glass coverslips in Petri dishes, as M(LPS), $\mathrm{M}(\mathrm{IL} 4)$, or $\mathrm{M}(0)$ and were coated with pairwise modified alginates as described above. After $48 \mathrm{~h}$ of incubation, the cells were stained for CD11c and CD206. These coverslips were imaged with an EVOS ${ }^{\circledR}$ FLoid $^{\circledR}$ Imaging Station (Life Technologies, Grand Island, NY, USA) using the red channel (excitation/emission 586/646 nm), blue channel $(390 / 446 \mathrm{~nm}$ ) and the green channel $(482 / 532 \mathrm{~nm}$ ).

\subsection{TNF- $\alpha$ Diffusion}

To test the diffusive properties of these pairwise modified alginates, microparticles were made using an electrostatic droplet generation technique. Using the same equipment and techniques previously described [9], microparticles with an average diameter of $685.15 \pm 14.20 \mu \mathrm{m}$ were made with unmodified alginate. Amino polystyrene particles, $10 \mathrm{mg}$, (Spherotech Inc., Lake Forest, IL, USA) were also labeled with $2 \mathrm{mg}$ mouse IgG (Thermo Scientific, Waltham, MA, USA) in PBS using $20 \mathrm{mg}$ of EDC. These labeled polystyrene particles were encapsulated in the unmodified alginate particles during electrostatic droplet generation using procedures developed by Kulseng et al. [42]. Approximately 200 particles were incubated with $2 \mathrm{~mL}$ of supernatant known to contain TNF- $\alpha$. This supernatant was collected from M(LPS) RAW 264.7 cells cultured at $\sim 182,000$ cells $/ \mathrm{cm}^{2}$ after two days of incubation. After four days of incubation with the particles, the supernatant was removed and compared to the original TNF- $\alpha$ supernatant using the TNF- $\alpha$ ELISA described in Section 2.8 to determine how much had diffused into the particles.

\subsection{Statistics and Data Analysis}

Statistical analysis was performed using $\mathrm{JMP}^{\circledR}$ statistical software (Cary, NC, USA). Statistical significance of the mean comparisons was determined by a two-way ANOVA. Pair-wise comparisons were analyzed with Tukey's honest significant difference test. Differences were considered statistically significant for $p<0.05$. 


\section{Results}

\subsection{Modification and Characterization}

After the combined modification of alginate using the molecules in Figure 1, the synthesized materials were characterized for their hydrophobicity, compression modulus and percent modification. Elemental analysis confirmed modification with $\% \mathrm{~N}$ values being greater for the modified alginates than unmodified (Figure 2A,B). The WCAs of the combined modified alginates fell in a range of $39.9^{\circ}-51.2^{\circ}$ (Figure $2 \mathrm{C}$ ), compared to the range of $49.7^{\circ}-61.0^{\circ}$ with the single modifications, and $54.1^{\circ} \pm 2.0^{\circ}$ for unmodified alginate(Figure 2D). This suggests the materials remained relatively hydrophilic. The compression moduli of the combined modified alginates ranged from 12.5-30.1 kPa (Figure 2E), which was similar to the range in compression modulus for the single modifications $(18.8-25.0 \mathrm{kPa})$, and of unmodified alginate $(25.3 \pm 5.4 \mathrm{kPa}$, Figure $2 \mathrm{~F})$.

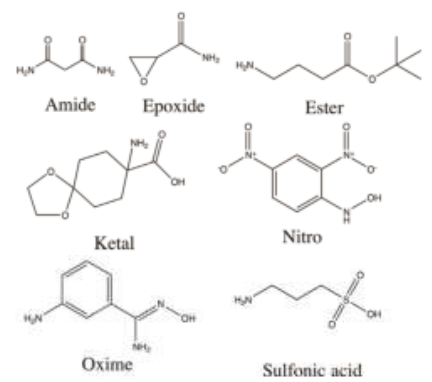

Figure 1. Chemical structures of the molecules used in the modification of medium viscosity alginate. The functional groups listed here are used as labels in the following figures for convenience.

A

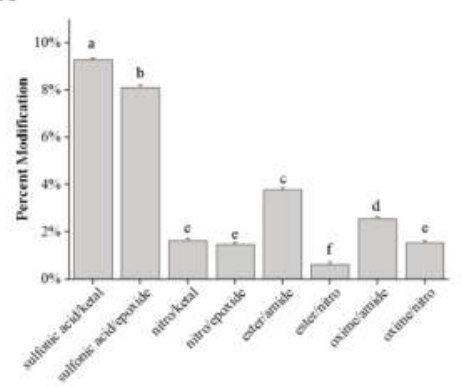

C

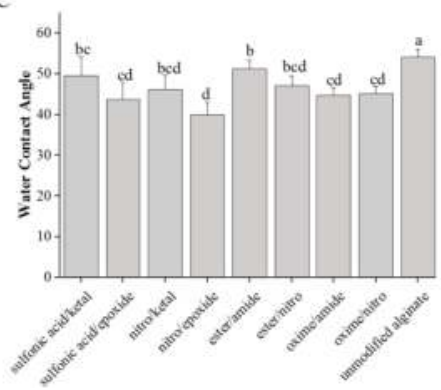

B

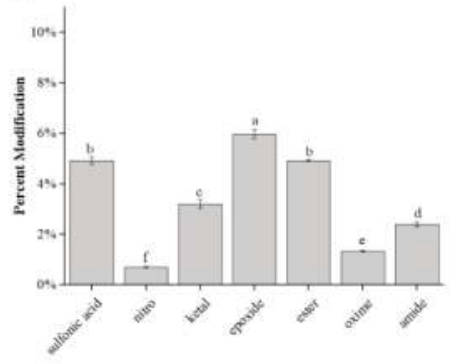

D

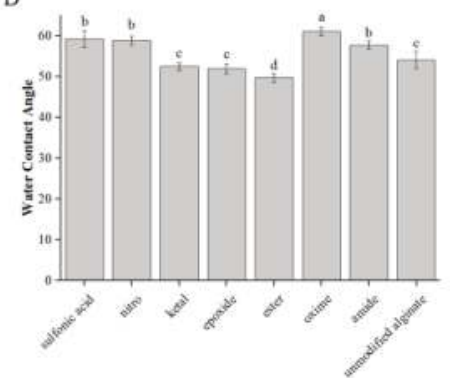

Figure 2. Cont. 
$\mathrm{E}$

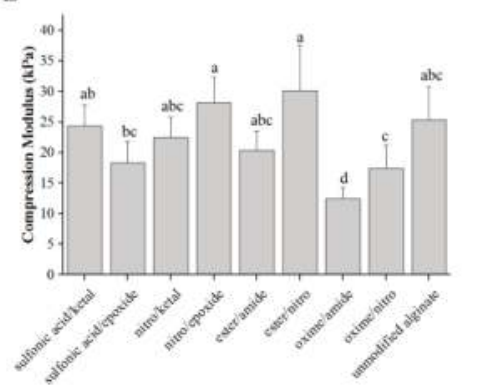

F

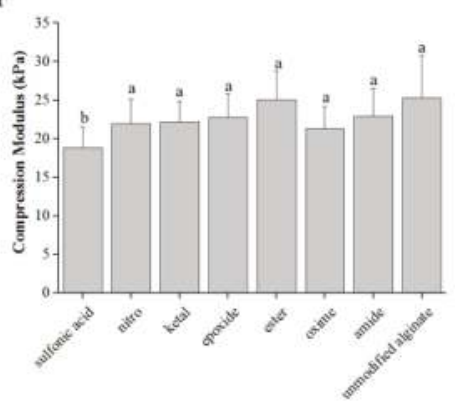

Figure 2. Characterization of combined and single modified alginates: $(\mathbf{A}, \mathbf{B})$ percent modifications; (C,D) WCAs; and (E,F) compression moduli were measured for double (A,C,E) and single (B,D,F) modifications. $n=5$. Data represent the mean value \pm standard deviation. Bars with the same letter (a-f) are not statistically different $(p<0.05)$.

\subsection{Cell Viability}

Biomaterials must be cytocompatible for use as implantable materials. Cytotoxicity of the combined modified alginates was measured using MTT assays. The viability of the cells was expressed as a percentage of the positive control of cells cultured on PLL coated tissue culture plastic. Cells exposed to all materials showed greater than $70 \%$ viability (Figure 3 ). This suggests minimal cytotoxicity, especially for cells cultured under a hydrogel.

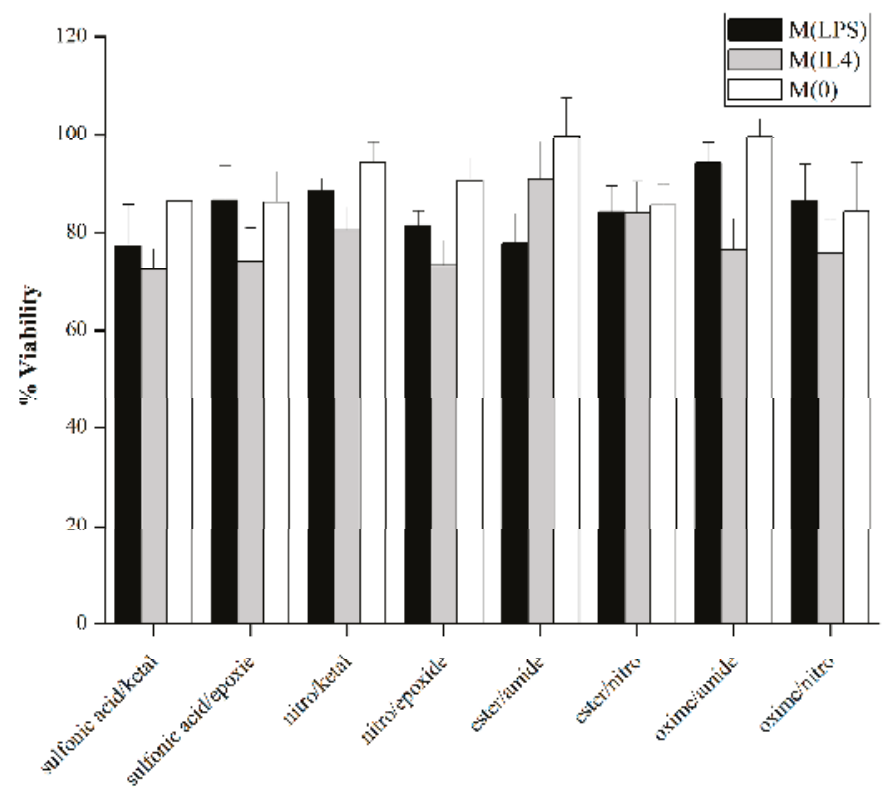

Figure 3. Modified alginates are cytocompaible. Cell viability of M(LPS), M(IL-4) and M(0) RAW 264.7 macrophages encapsulated under modified and unmodified alginate layers. $n=4$. Data represent the mean value \pm standard deviation. 


\subsection{Arginase/iNOS}

Arginase activity was determined for encapsulated macrophages by exposing cell lysate to arginine and quantifying the urea produced. Nitrites are the stable form of $\mathrm{NO}$ and were quantified through a Griess reagent assay. Absolute values for both urea and Griess assays are given in Figure 4A-D, with the pairwise modifications on the left and the single modifications on the right for comparison. Results from these assays are given as urea: nitrite in Figure $4 \mathrm{E}, \mathrm{F}$, which corresponds to the amount of urea produced divided by the amount of nitrite in corresponding samples. Ratio values range from $7.12 \mathrm{mg} / \mu \mathrm{mol}$ with sulfonic acid/ ketal to $36.2 \mathrm{mg} / \mu \mathrm{mol}$ with ester/nitro for M(IL-4) macrophages. A value of $6.88 \pm 3.05 \mathrm{mg} / \mu \mathrm{mol}$ was previously determined for M(IL-4) macrophages in the presence of unmodified alginate [9]. M(LPS) macrophages exhibited a narrow range of 6.20 to $14.1 \mathrm{mg} / \mu \mathrm{mol}$ compared to $7.52 \pm 1.76 \mathrm{mg} / \mu \mathrm{mol}$ for unmodified alginate. Finally, values for $\mathrm{M}(0)$ macrophages ranged from $4.24 \mathrm{mg} / \mu \mathrm{mol}$ with the oxime/nitro modification to $33.8 \mathrm{mg} / \mu \mathrm{mol}$ with ester/nitro. $\mathrm{M}(0)$ macrophages exhibited a urea:nitrite ratio of $6.91 \pm 2.13 \mathrm{mg} / \mu \mathrm{mol}$ with unmodified alginate. Higher values for these ratios indicate a stronger M2 phenotype presence, while low values suggest a M1 phenotype. Most values seem to indicate a stronger M2 presence when compared to unmodified alginate. Exceptions to this statement include oxime/amide and oxime/nitro M(LPS) macrophages.

The controls for M(IL-4), M(LPS), and M(0) cells were higher than the cells exposed to the modified and unmodified alginates with $124 \pm 30.6,13.1 \pm 2.38$ and $94.3 \pm 29.5 \mathrm{mg} / \mu \mathrm{mol}$ being measured, respectively. The urea measured for the controls was in line with the values measured for the cells exposed to alginate samples-20.1 $\pm 3.25,16.4 \pm 2.97$ and $6.31 \pm 1.14 \mathrm{mg} / \mathrm{dL}$ for M(IL-4), M(LPS), and $\mathrm{M}(0)$ cells, respectively. However, the nitrite levels were much lower for M(IL-4) and M(0) cells, leading to a large increase in the urea:nitrite ratio. The measured nitrite levels were $1.62 \pm 0.30$, $12.5 \pm 0.25$ and $0.67 \pm 0.17 \mu \mathrm{M}$ for $\mathrm{M}(\mathrm{IL}-4), \mathrm{M}(\mathrm{LPS})$, and $\mathrm{M}(0)$ cells respectively.

A

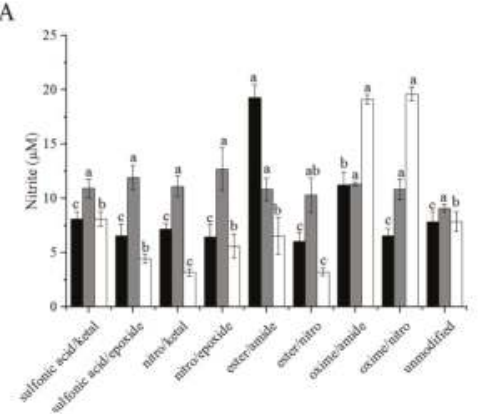

$\mathrm{C}$

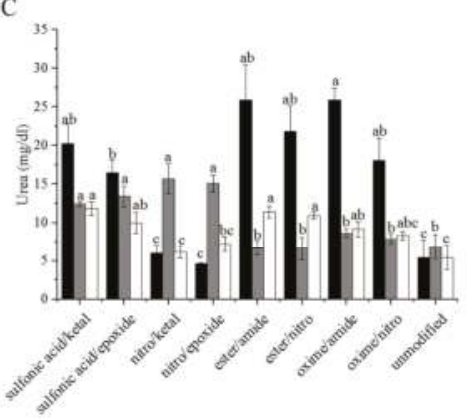

B

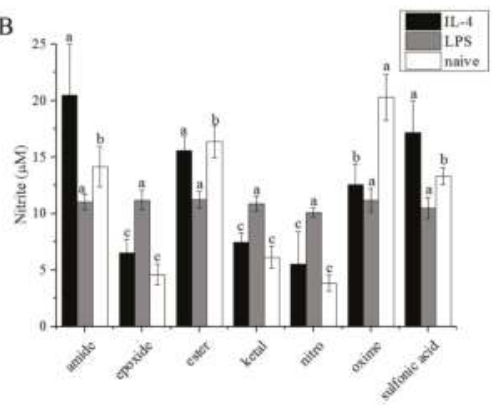

D

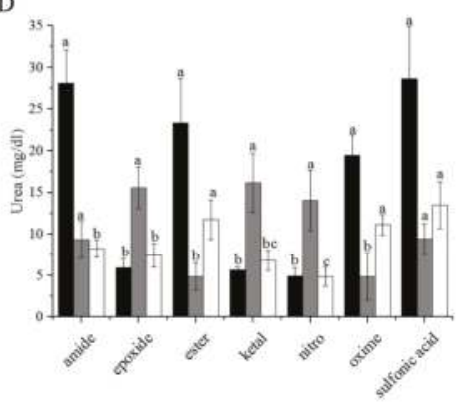

Figure 4. Cont. 
E
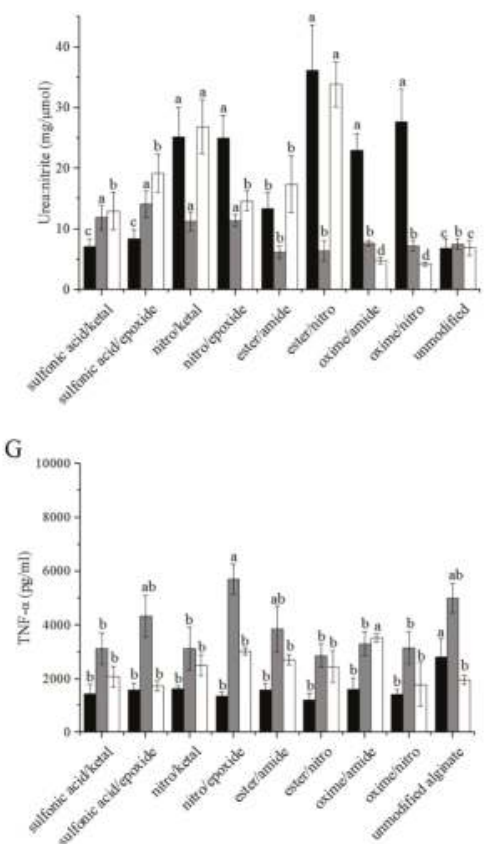

$\mathrm{F}$

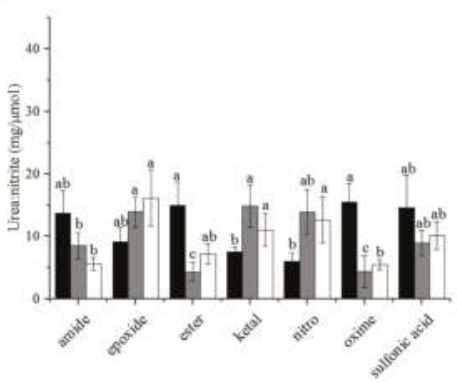

$\mathrm{H}$

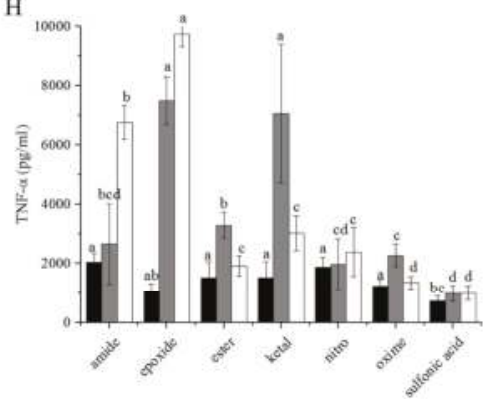

Figure 4. Molecules produced by polarized RAW 264.7 macrophages encapsulated under modified alginates. Alginates were layered over activated macrophages and: (A,B) nitrite; (C,D) urea; (E,F) urea:nitrite; and $(\mathbf{G}, \mathbf{H})$ TNF- $\alpha$ were measured for dual $(\mathbf{A}, \mathbf{C}, \mathbf{E}, \mathbf{G})$ and single $(\mathbf{B}, \mathbf{D}, \mathbf{F}, \mathbf{H})$ modifications. Arginase activity was measured by removing the alginate layer and lysing the cells. $n=4$. Data represent the mean value \pm standard deviation. Bars with the same letter $(\mathrm{a}-\mathrm{d})$ are not statistically different $(p<0.05)$ from data points of the same activation.

\subsection{TNF- $\alpha$ ELISA}

Production of the cytotoxic cytokine TNF- $\alpha$ by cells exposed to modified alginates was measured and reported in Figure 4G. Values for single modifications of alginate are included in Figure $4 \mathrm{H}$ for comparison. Most modifications of alginate were able to decrease the amount of TNF- $\alpha$ produced in comparison to unmodified alginate for all macrophage phenotypes. The exception to this statement is oxime/amide for $\mathrm{M}(0)$ cells. TNF- $\alpha$ production by $\mathrm{M}(\mathrm{IL}-4)$ macrophages ranged from 1.2 to $1.6 \mathrm{ng} / \mathrm{mL}$ with $2.8 \pm 0.68 \mathrm{ng} / \mathrm{mL}$ produced under unmodified alginate. $\mathrm{M}(0)$ macrophages produced a range of TNF- $\alpha$ that spanned 1.8 to $3.5 \mathrm{ng} / \mathrm{mL}$ with unmodified alginate eliciting $1.9 \pm 0.17 \mathrm{ng} / \mathrm{mL}$ TNF- $\alpha$. TNF- $\alpha$ produced by M(LPS) cells spanned a higher range of 2.9 to $5.7 \mathrm{ng} / \mathrm{mL}$, and on a sample-to-sample basis, the amount of cytokine produced was most often highest for M(LPS) macrophages. In the presence of unmodified alginate, M(LPS) cells produced $5.0 \pm 0.53 \mathrm{ng} / \mathrm{mL}$ of TNF- $\alpha$. All modifications had statistically lower TNF- $\alpha$ production than unmodified alginate for $\mathrm{M}(\mathrm{IL}-4)$ macrophages. Similar to nitrite, TNF- $\alpha$ secretion from M(IL-4) and M(0) cells was much lower for the control than for the cells in the presence of alginate. The values for the controls were $0.58 \pm 0.066,5.0 \pm 0.35$ and $0.39 \pm 0.016 \mathrm{ng} / \mathrm{mL}$ for M(IL-4), M(LPS) and M(0) cells, respectively.

\subsection{Fluorescent Imaging}

Immunocytochemistry (ICC) fluorescent staining was used to identify the macrophage phenotypes resulting from culturing cells in the presence of modified alginates. CD11c, an LPS receptor, was used 
to identify M1 macrophages and a CD206, a mannose receptor, was used to indicate an M2 macrophage phenotype. DAPI was also used as a nuclei stain. Representative images are shown in Figure 5A,B. These images were used to qualitatively support the results found by measuring arginase activity, TNF- $\alpha$ secretion, and nitrite production. Control staining images of macrophages with activator alone are also included in Figure 5C.

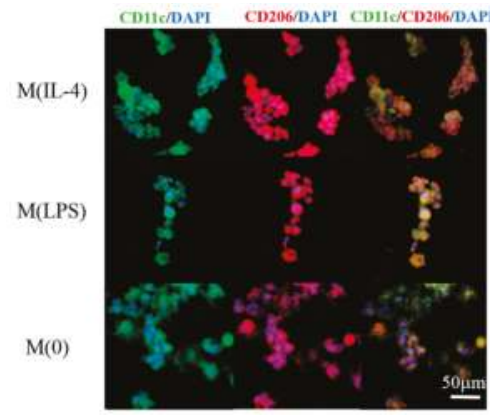

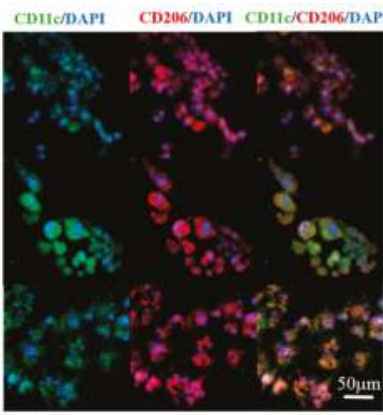

B

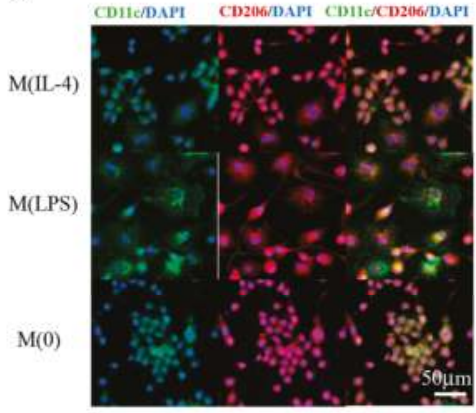

C

Figure 5. Immunocytochemistry staining of macrophages suggests that modified alginates alter phenotype: (A) nitro/epoxide; and (B) ester/amide modified alginates were coated on cells; and $(\mathbf{C})$ cells in the absence of alginate are shown for comparison. Cells were stained with fluorescently labeled CD206 (red, M2 marker) and CD11c (green, M1 marker) markers as well as DAPI (blue). Scale bar is $50 \mu \mathrm{m}$.

\subsection{Synergy}

Normalizing the percent modifications of each combined alginate sample to that of the single modifications, we were able to determine weight fractions of each modification. Synergy was calculated using the equation that was developed by Karande et al. [43]:

$$
S=\frac{m_{\mathrm{A}+\mathrm{B}}}{x m_{\mathrm{A}}+(1-x) m_{\mathrm{B}}}
$$

where $m_{\mathrm{A}}, m_{\mathrm{B}}$, and $m_{\mathrm{A}+\mathrm{B}}$ are the measured values for the different modifications and $x$ is the weight fraction of each modification determined by elemental analysis. These values are shown for each macrophage phenotype in Figure 6A-D. The synergy values for nitrite were nearly one for M(LPS) cells exposed to all modifications (0.930-1.147). Synergy values were slightly more dynamic for M(IL-4) cells, ranging from 0.578 to 1.121 , and for $\mathrm{M}(0)$ cells, ranging from 0.218 to 1.34 . For urea, all of the synergy values for all of the macrophage activation states were near unity. This resulted in nitrite being entirely responsible for the synergy values for urea: nitrite. Since M(LPS) cells did not have large 
variation in the synergy values for the amount of nitrite measured in the supernatant, the synergy values were correspondingly nearly unity $(0.854-1.159)$. Both $\mathrm{M}(\mathrm{IL}-4)$ and $\mathrm{M}(0)$ cells yielded more dynamic synergy values for urea: nitrite of 0.811 to 2.613 for M(IL-4) and 0.533 to 4.31 for M(0). Finally, the synergy values for TNF- $\alpha$ production were in the range of $0.783-1.778$ for M(IL-4) macrophages, $0.511-1.453$ for M(LPS) macrophages, and $0.303-1.246$ for $\mathrm{M}(0)$ macrophages.

A

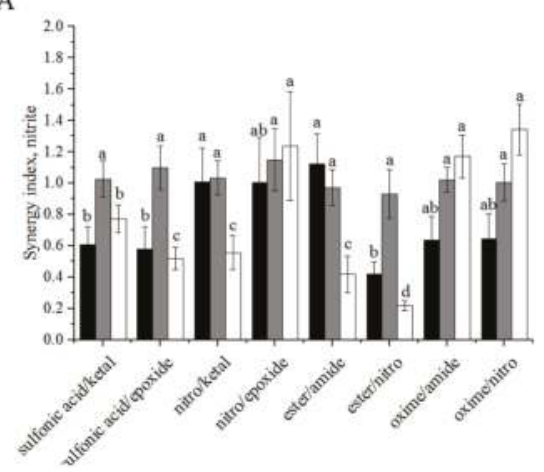

$\mathrm{C}$

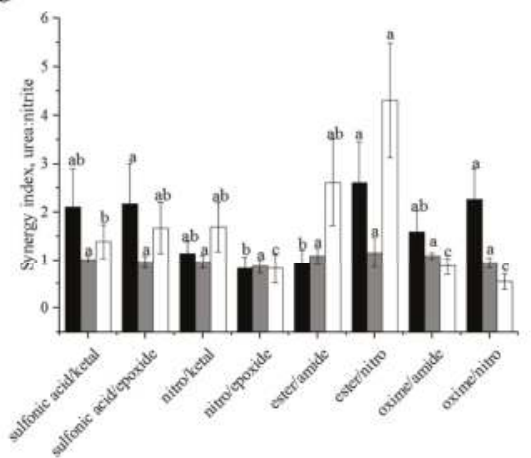

B

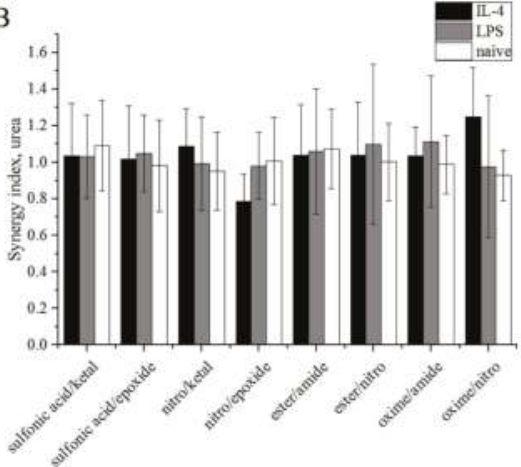

D

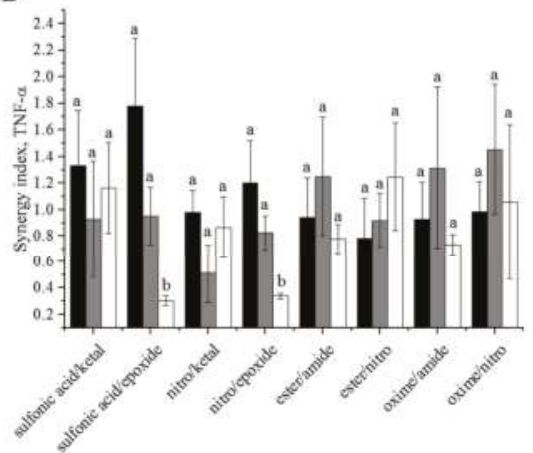

Figure 6. Synergistic indices for molecules produced by polarized RAW 264.7 macrophages: (A) nitrite; (B) urea; (C) urea:nitrite; and (D) TNF- $\alpha$ for M(LPS), M(IL-4), and M(0) cells were calculated based on previously obtained values for single modifications of alginates [9]. $n=4$. Data represent the mean value \pm standard deviation. Bars with the same letter $(a-d)$ are not statistically different $(p<0.05)$ from data points of the same activation. All measured values in panel B were statistically similar.

\subsection{TNF- $\alpha$ Diffusion}

The amount of TNF- $\alpha$ able to diffuse into the particles was measured using an ELISA after four days of incubation (Figure 7A) [9]. A wide range of values were calculated for percent loading. Nitro/ketal did not allow any diffusion of TNF- $\alpha$, while nitro/epoxide allowed $87 \%$ loading. Percent loading values for single modifications of alginate are included in Figure 7B for comparison. Synergy values for TNF- $\alpha$ diffusion were calculated in the range of 0 to 4.94 (Figure 7C). 


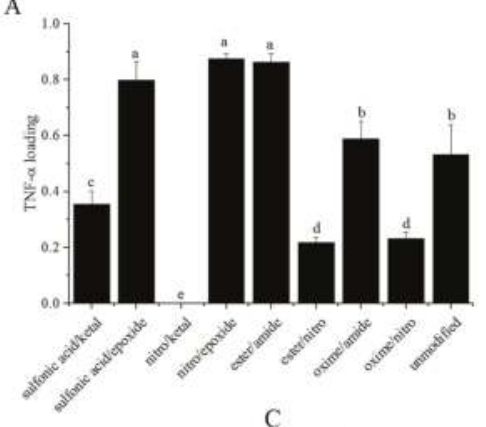

B

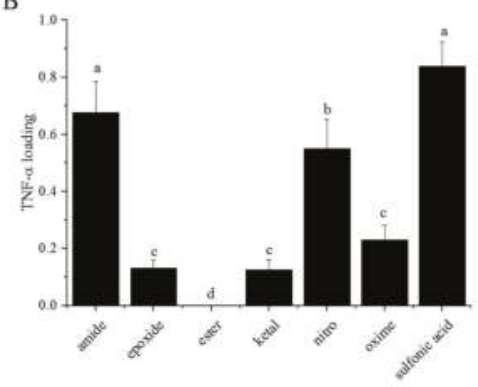

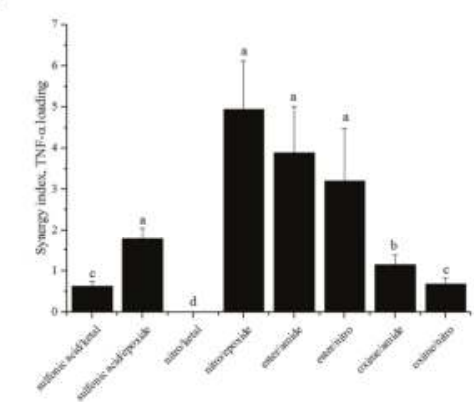

Figure 7. Modified alginates alter mass transport of TNF- $\alpha$ through layered alginate particles. (A) Combined; and (B) single modified alginates were layered over alginate particles containing mouse IgG labeled polystyrene particles. $n=4$. Data represent the mean value \pm standard deviation. Bars with the same letter $(\mathrm{a}-\mathrm{d})$ are not statistically different $(p<0.05)$ from data points of the same activation.

\section{Discussion}

Alginate is an appealing biomaterial for numerous wound healing and tissue engineering applications. It is a naturally occurring, relatively inexpensive polymer, it has low toxicity and good biocompatibility, and is easily gelled under mild conditions with divalent cations [44]. The structure of alginate is similar to that of the extracellular matrix in living tissues, and it provides a relatively inert and moist microenvironment [44,45] However, the biocompatibility of alginate remains insufficiently understood. For example, in research by Elliot et al., alginate was used to encapsulate porcine islets in a clinical trial for diabetes treatment [46]. While the trials were mildly successful in that there were surviving functional islets, the overall function of the implant was severely diminished by the presence of granulation tissue that developed in response to the alginate [46]. Many aspects of the polymer have been studied in an attempt to improve upon the biocompatibility [47-60], and some work has been done to chemically modify alginate and study the impact this has on cell function [9,61-63]. This research focuses on combining surface modifications to study the synergistic effects of chemical modification on cellular responses. By using materials that have been studied in the past, we have been able to compare predicted and actual values to examine synergy of the modifications.

In this study, we aimed to find combined modifications that would improve the biocompatibility of alginate by reprogramming macrophages towards an M2-like phenotype. Based on the equation used to calculate synergy, values greater than one for urea:nitrite and less than one for both TNF- $\alpha$ and nitrite production would be desired. These values are most important for M(LPS) macrophages as this is the phenotype we are aiming to reprogram. M(LPS) macrophages tend to mediate the inflammatory stages of the foreign body response to biomaterials, which we are attempting to minimize. TNF- $\alpha$ production synergy values tended to be less than or equal to one for all phenotypes studied. This may 
be due, in part, to the fact the TNF- $\alpha$, must diffuse through the alginate coating in order to be measured. As Figure 7 indicates, some of these combined modifications allow little to no TNF- $\alpha$ diffusion through the alginate. However, combining this information with the urea synergy values, we can determine which modifications may be favorably shifting macrophage phenotype towards an M2-like state. From the values presented in Figure 6, it can be determined that almost all the modifications do not result in cooperative interactions with M(LPS) macrophages. This is particularly interesting and somewhat surprising for TNF- $\alpha$ production since variable diffusion of TNF- $\alpha$ through the different modifications was observed (Figure 7).

Several different observations were noticed when examining synergistic values of TNF- $\alpha$ production in concert with urea:nitrite. With respect to the $\mathrm{M}(0)$ macrophage synergy values, sulfonic acid/epoxide and nitro/epoxide resulted in antagonistic synergy values for TNF- $\alpha$ production. Perhaps unsurprisingly, the pairwise modifications with epoxide resulted in increases in TNF- $\alpha$ production synergy values for M(IL-4) cells. Epoxide was chosen for this study because it resulted in low permeability and high TNF- $\alpha$ production. Sulfonic acid combinations are also able to increase TNF- $\alpha$ production synergy values for M(IL-4) cells and increased urea:nitrite values. Sulfonic acid/epoxide was also able to improve urea: nitrite values, indicating the combinations of these modifications shifted $\mathrm{M}(0)$ cells towards an $\mathrm{M} 2$ phenotype. Ester combinations resulted in improved urea:nitrite values and did not have an observable synergistic effect on TNF- $\alpha$ for $\mathrm{M}(0)$ cells. Future work in examining the synergy of these materials in a dose dependent context may elucidate the relationship of these modifications on shifting macrophage phenotypes.

By including the TNF- $\alpha$ diffusion study, this work also examined the changes in diffusive properties of alginate due to combined surface modifications. Ideally, combined modifications would result in a lower mass transport of TNF- $\alpha$ than in the case of single modifications. This would be indicated by synergy values less than one. Modifications sulfonic acid/ketal, nitro/ketal, and oxime/nitro all had values below one. Ketal lowering diffusion was expected since it was previously observed to have low permeability for TNF- $\alpha$ [9]. Interestingly, when ketal was combined with nitro or sulfonic acid, the synergy values were less than one even though nitro and sulfonic acid were selected for their poor permselectivity towards TNF- $\alpha$ and low production of TNF- $\alpha$. The other modifications had relatively high synergy values, suggesting that they are not permselective towards TNF- $\alpha$, which could potentially be harmful for islet encapsulation as in Elliot et al. [46]. In that example, tissue development surrounding the implant cut off blood supply to the encapsulated islet preventing them from functioning properly. In this case, with increased TNF- $\alpha$ release, the toxic cytokine would be able to reach the islets and impact their ability to regulate blood sugar.

\section{Conclusions}

Pair-wise modifications of alginate resulted in synergistic and antagonistic responses for M(IL-4), $\mathrm{M}(\mathrm{LPS})$ and $\mathrm{M}(0)$ cells for both nitrite and TNF- $\alpha$ secretion. There was no observed advantage or disadvantage of these modifications in terms of arginase activity. In combining the data measuring the shifts in macrophage phenotype with that examining TNF- $\alpha$ diffusion through the modified alginates, improved materials for artificial organs can be obtained. Interestingly, certain modifications appear to influence the synergistic index for TNF- $\alpha$ diffusion, such as ketal, which lowered the index for both pair-wise combinations in which it was included. This observation held for material mediated shifts in macrophage phenotype, e.g., ester for TNF- $\alpha$ secretion from $\mathrm{M}(0)$ cells. These results further demonstrate the importance of chemical moieties on biomaterials in achieving appropriate cellular responses. Although this study is not conclusive, it provides valuable insight into the impact of multiple surface modifications on macrophage phenotype. This library is also not large enough to offer much in the way of justified reasoning for the synergistic effects of the combined modifications. However, it provided evidence that maximum macrophage reprogramming may require a multitude of stimuli, and further supported the findings that simply altering the surface chemistry of a biomaterial may have a significant impact on its biocompatibility. 
Acknowledgments: This work was supported by the National Science Foundation under Grant No. CBET-1227867 and the Roy J. Carver Charitable Trust Grant No. 13-4265. The authors also acknowledge support from NSF ARI-R2 (CMMI-0963224) for funding the renovation of the research laboratories used for these studies.

Author Contributions: Hannah C. Bygd and Kaitlin M. Bratlie designed the study. Hannah C. Bygd performed the experiments. Hannah C. Bygd and Kaitlin M. Bratlie analyzed data. Hannah C. Bygd and Kaitlin M. Bratlie wrote the manuscript.

Conflicts of Interest: The authors declare no conflict of interest.

\section{References}

1. Mantovani, A.; Sica, A. Macrophages, innate immunity and cancer: Balance, tolerance, and diversity. Curr. Opin. Immunol. 2010, 22, 231-237. [CrossRef] [PubMed]

2. Jain, S.; Tran, T.-H.; Amiji, M. Macrophage repolarization with targeted alginate nanoparticles containing IL-10 plasmid DNA for the treatment of experimental arthritis. Biomaterials 2015, 61, 162-177. [CrossRef] [PubMed]

3. Quatromoni, J.G.; Eruslanov, E. Tumor-associated macrophages: Function, phenotype, and link to prognosis in human lung cancer. Am. J. Transl. Res. 2012, 4, 376-389. [PubMed]

4. Wynn, T.A.; Barron, L.; Thompson, R.W.; Madala, S.K.; Wilson, M.S.; Cheever, A.W.; Ramalingam, T. Quantitative assessment of macrophage functions in repair and fibrosis. Curr. Protoc. Immunol. 2011, 2011. [CrossRef]

5. Mantovani, A.; Biswas, S.K.; Galdiero, M.R.; Sica, A.; Locati, M. Macrophage plasticity and polarization in tissue repair and remodelling. J. Pathol. 2013, 229, 176-185. [CrossRef] [PubMed]

6. Peer, D.; Karp, J.M.; Hong, S.; Farokhzad, O.C.; Margalit, R.; Langer, R. Nanocarriers as an emerging platform for cancer therapy. Nat. Nanotechnol. 2007, 2, 751-760. [CrossRef] [PubMed]

7. Kelly, C.; Jefferies, C.; Cryan, S. Targeted liposomal drug delivery to monocytes and macrophages. J. Drug Deliv. 2011, 2011, 727241. [CrossRef] [PubMed]

8. Bygd, H.C.; Forsmark, K.D.; Bratlie, K.M. The significance of macrophage phenotype in cancer and biomaterials. Clin. Transl. Med. 2014, 3. [CrossRef] [PubMed]

9. Bygd, H.C.; Bratlie, K.M. The effect of chemically modified alginates on macrophage phenotype and biomolecule transport. J. Biomed. Mater. Res. Part A 2016, 104, 1707-1719. [CrossRef] [PubMed]

10. Xia, Z.; Triffitt, J.T. A review on macrophage responses to biomaterials. Biomed. Mater. 2006, 1, R1-R9. [CrossRef] [PubMed]

11. Ward, W.K. A Review of the Foreign-body Response to Subcutaneously-implanted Devices: The Role of Macrophages and Cytokines in Biofouling and Fibrosis. J. Diabetes Sci. Technol. 2008, 2, 768-777. [CrossRef]

12. Murray, P.J.; Allen, J.E.; Biswas, S.K.; Fisher, E.A.; Gilroy, D.W.; Goerdt, S.; Gordon, S.; Hamilton, J.A.; Ivashkiv, L.B.; Lawrence, T.; et al. Macrophage Activation and polarization: Nomenclature and experimental guidelines. Immunity 2014, 41, 14-20. [CrossRef] [PubMed]

13. Martinez, F.O.; Gordon, S. The M1 and M2 paradigm of macrophage activation: Time for reassessment. F1000Prime Rep. 2014, 6, 13. [CrossRef] [PubMed]

14. Akilbekova, D.; Philiph, R.; Graham, A.; Bratlie, K.M. Macrophage reprogramming: Influence of latex beads with various functional groups on macrophage phenotype and phagocytic uptake in vitro. J. Biomed. Mater. Res. Part A 2015, 103, 262-268. [CrossRef] [PubMed]

15. Bygd, H.C.; Forsmark, K.D.; Bratlie, K.M. Altering in vivo macrophage responses with modified polymer properties. Biomaterials 2015, 56, 187-197. [CrossRef] [PubMed]

16. Sica, A.; Mantovani, A. Macrophage plasticity and polarization: In vivo veritas. J. Clin. Investig. 2012, 122, 787-795. [CrossRef] [PubMed]

17. Rodriguez, A.; Meyerson, H.; Anderson, J.M. Quantitative in vivo cytokine analysis at synthetic biomaterial implant sites. J. Biomed. Mater. Res. A 2009, 89, 152-159. [PubMed]

18. Suwandi, J.S.; Toes, R.E.M.; Nikolic, T.; Roep, B.O. Inducing tissue specific tolerance in autoimmune disease with tolerogenic dendritic cells. Clin. Exp. Rheumatol. 2015, 33, 97-103.

19. Mesure, L.; De Visscher, G.; Vranken, I.; Lebacq, A.; Flameng, W. Gene expression study of monocytes/ macrophages during early foreign body reaction and identification of potential precursors of myofibroblasts. PLoS ONE 2010, 5, e12949. [CrossRef] [PubMed] 
20. Gordon, S. Alternative activation of macrophages. Nat. Rev. Immunol. 2003, 3, 23-35. [CrossRef] [PubMed]

21. Gordon, S.; Martinez, F.O. Alternative activation of macrophages: Mechanism and functions. Immunity 2010, 32, 593-604. [CrossRef] [PubMed]

22. Yang, J.; Mei, Y.; Hook, A.L.; Taylor, M.; Urquhart, A.J.; Bogatyrev, S.R.; Langer, R.; Anderson, D.G.; Davies, M.C.; Alexander, M.R. Polymer surface functionalities that control human embryoid body cell adhesion revealed by high throughput surface characterization of combinatorial material microarrays. Biomaterials 2010, 31, 8827-8838. [CrossRef] [PubMed]

23. McNally, A.K.; Anderson, J.M. Complement C3 participation in monocyte adhesion to different surfaces. Proc. Natl. Acad. Sci. USA 1994, 91, 10119-10123. [CrossRef] [PubMed]

24. Yang, D.; Jones, K.S. Effect of alginate on innate immune activation of macrophages. J. Biomed. Mater. Res. A 2009, 90, 411-418. [CrossRef] [PubMed]

25. MacLauchlan, S.; Skokos, E.A.; Meznarich, N.; Zhu, D.H.; Raoof, S.; Shipley, J.M.; Senior, R.M.; Bornstein, P.; Kyriakides, T.R. Macrophage fusion, giant cell formation, and the foreign body response require matrix metalloproteinase 9. J. Leukoc. Biol. 2009, 85, 617-626. [CrossRef] [PubMed]

26. Mosser, D.M.; Edwards, J.P. Exploring the full spectrum of macrophage activation. Nat. Rev. Immunol. 2008, 8, 958-969. [CrossRef] [PubMed]

27. Wang, Y.; Wang, Y.P.; Zheng, G.; Lee, V.W.S.; Ouyang, L.; Chang, D.H.H.; Mahajan, D.; Coombs, J.; Wang, Y.M.; Alexander, S.I.; et al. Ex vivo programmed macrophages ameliorate experimental chronic inflammatory renal disease. Kidney Int. 2007, 72, 290-299. [CrossRef] [PubMed]

28. Anderson, J.M. Biological responses to materials. Annu. Rev. Mater. Res. 2001, 31, 81-110. [CrossRef]

29. Lu, Y.; Huang, J.; Yu, G.; Cardenas, R.; Wei, S.; Wujcik, E.K.; Guo, Z. Coaxial electrospun fibers: Applications in drug delivery and tissue engineering. Wiley Interdiscip. Rev. Nanomed. Nanobiotechnol. 2016, 8, 654-677. [CrossRef] [PubMed]

30. Wujcik, E.K.; Monty, C.N. Nanotechnology for implantable sensors: Carbon nanotubes and graphene in medicine. Wiley Interdiscip. Rev. Nanomed. Nanobiotechnol. 2013, 5, 233-249. [CrossRef] [PubMed]

31. Panilaitis, B.; Altman, G.H.; Chen, J.; Jin, H.J.; Karageorgiou, V.; Kaplan, D.L. Macrophage responses to silk. Biomaterials 2003, 24, 3079-3085. [CrossRef]

32. Wang, D.; Bratlie, K.M. Influence of polymer chemistry on cytokine secretion from polarized macrophages. ACS Biomater. Sci. Eng. 2015, 1, 166-174. [CrossRef]

33. Wang, D.; Phan, N.; Isely, C.; Bruene, L.; Bratlie, K.M. Effect of surface modification and macrophage phenotype on particle internalization. Biomacromolecules 2014, 15, 4102-4110. [CrossRef] [PubMed]

34. Goldberg, M.; Mahon, K.; Anderson, D. Combinatorial and rational approaches to polymer synthesis for medicine. Adv. Drug Deliv. Rev. 2008, 60, 971-978. [CrossRef] [PubMed]

35. Brocchini, S. Combinatorial chemistry and biomedical polymer development. Adv. Drug Deliv. Rev. 2001, 53, 123-130. [CrossRef]

36. Liu, E.; Treiser, M.D.; Patel, H.; Sung, H.J.; Roskov, K.E.; Kohn, J.; Becker, M.L.; Moghe, P.V. High-content profiling of cell responsiveness to graded substrates based on combinatorially variant polymers. Comb. Chem. High Throughput Screen. 2009, 12, 645-655. [CrossRef]

37. Tallarida, R.J. Drug synergism: Its detection and applications. J. Pharmacol. Exp. Ther. 2001, 298, 865-872. [PubMed]

38. Mitragotri, S. Synergistic effect of enhancers for transdermal drug delivery. Pharm. Res. 2000, 17, 1354-1359. [CrossRef] [PubMed]

39. Chou, T.C. Drug combination studies and their synergy quantification using the chou-talalay method. Cancer Res. 2010, 70, 440-446. [CrossRef] [PubMed]

40. Lehár, J.; Krueger, A.S.; Avery, W.; Heilbut, A.M.; Johansen, L.M.; Price, E.R.; Rickles, R.J.; Short, G.F.; Staunton, J.E.; Jin, X.; et al. Synergistic drug combinations tend to improve therapeutically relevant selectivity. Nat. Biotechnol. 2009, 27, 659-666. [CrossRef] [PubMed]

41. Levinson, S.S. Kinetic centrifugal analyzer and manual determination of serum urea nitrogen, with use of o-phthaldialdehyde reagent. Clin. Chem. 1978, 24, 2199-2202. [PubMed]

42. Kulseng, B.; Thu, B.; Espevik, T.; Skjåk-Braek, G. Alginate poly-lysine microcapsules as immune barrier: Permeability of cytokines and immunoglobins over the capsule membrane. Cell Transplant. 1997, 6, 387-394. [CrossRef] 
43. Karande, P.; Jain, A.; Mitragotri, S. Insights into synergistic interactions in binary mixtures of chemical permeation enhancers for transdermal drug delivery. J. Control. Release 2006, 115, 85-93. [CrossRef] [PubMed]

44. Lee, K.Y.; Mooney, D.J. Alginate: Properties and biomedical applications. Prog. Polym. Sci. 2012, 37, 106-126. [CrossRef] [PubMed]

45. Gombotz, W.R.; Wee, S.F. Protein release from alginate matrices. Adv. Drug Deliv. Rev. 2012, 64, 194-205. [CrossRef]

46. Elliott, R.B.; Escobar, L.; Tan, P.L.; Muzina, M.; Zwain, S.; Buchanan, C. Live encapsulated porcine islets from a type 1 diabetic patient $9.5 \mathrm{yr}$ after xenotransplantation. Xenotransplantation 2007, 14, 157-161. [CrossRef] [PubMed]

47. De Vos, P.; van Hoogmoed, C.G.; van Zanten, J.; Netter, S.; Strubbe, J.H.; Busscher, H.J. Long-term biocompatibility, chemistry, and function of microencapsulated pancreatic islets. Biomaterials 2003, 24, 305-312. [CrossRef]

48. Tam, S.K.; Bilodeau, S.; Dusseault, J.; Langlois, G.; Hallé, J.P.; Yahia, L.H. Biocompatibility and physicochemical characteristics of alginate-polycation microcapsules. Acta Biomater. 2011, 7, 1683-1692. [CrossRef] [PubMed]

49. De Vos, P.; De Haan, B.; Van Schilfgaarde, R. Effect of the alginate composition on the biocompatibility of alginate-polylysine microcapsules. Biomaterials 1997, 18, 273-278. [CrossRef]

50. Mallett, A.G.; Korbutt, G.S. Alginate modification improves long-term survival and function of transplanted encapsulated islets. Tissue Eng. Part A 2009, 15, 1301-1309. [CrossRef] [PubMed]

51. Chen, J.-P.; Chu, I.-M.; Shiao, M.-Y.; Hsu, B.R.-S.; Fu, S.-H. Microencapsulation of islets in PEG-amine modified alginate-poly(L-Lysine )-alginate microcapsules for constructing bioartificial pancreas. J. Ferment. Bioeng. 1998, 86, 185-190. [CrossRef]

52. Jork, A.; Thürmer, F.; Cramer, H.; Zimmermann, G.; Gessner, P.; Hämel, K.; Hofmann, G.; Kuttler, B.; Hahn, H.J.; Josimovic-Alasevic, O.; et al. Biocompatible alginate from freshly collected Laminaria pallida for implantation. Appl. Microbiol. Biotechnol. 2000, 53, 224-229. [CrossRef] [PubMed]

53. Petruzzo, P.; Cappai, A.; Ruiu, G.; Dessy, E.; Rescigno, A.; Brotzu, G. Development of biocompatible barium alginate microcapsules. Transplant. Proc. 1997, 1345, 2129-2130. [CrossRef]

54. Schneider, S.; Feilen, P.J.; Kraus, O.; Haase, T.; Sagban, T.A.; Lehr, H.A.; Beyer, J.; Pommersheim, R.; Weber, M.M. Biocompatibility of alginates for grafting: Impact of alginate molecular weight. Artif. Cells Blood Substit. Biotechnol. 2003, 31, 383-394. [CrossRef]

55. Zimmermann, U.; Thürmer, F.; Jork, A.; Weber, M.; Mimietz, S.; Hillgärtner, M.; Brunnenmeier, F.; Zimmermann, H.; Westphal, I.; Fuhr, G.; et al. A novel class of amitogenic alginate microcapsules for long-term immunoisolated transplantation. Ann. N. Y. Acad. Sci. 2006, 944, 199-215. [CrossRef]

56. Steele, J.A.M.; Hallé, J.-P.; Poncelet, D.; Neufeld, R.J. Therapeutic cell encapsulation techniques and applications in diabetes. Adv. Drug Deliv. Rev. 2014, 67-68, 74-83. [CrossRef] [PubMed]

57. Paredes-Juarez, G.; de Haan, B.; Faas, M.; de Vos, P. A technology platform to test the efficacy of purification of alginate. Materials 2014, 7, 2087-2103. [CrossRef]

58. Calafiore, R.; Basta, G. Clinical application of microencapsulated islets: Actual prospectives on progress and challenges. Adv. Drug Deliv. Rev. 2014, 67-68, 84-92. [CrossRef] [PubMed]

59. Lee, C.; Shin, J.; Lee, J.S.; Byun, E.; Ryu, J.H.; Um, S.H.; Kim, D.I.; Lee, H.; Cho, S.W. Bioinspired, calcium-free alginate hydrogels with tunable physical and mechanical properties and improved biocompatibility. Biomacromolecules 2013, 14, 2004-2013. [CrossRef] [PubMed]

60. Orive, G.; Hernandez, R.M.; Gascon, A.R. Survival of different cell lines in alginate-agarose microcapsules. Eur. J. Pharm. Sci. 2003, 18, 23-30. [CrossRef]

61. Yang, J.-S.; Xie, Y.-J.; He, W. Research progress on chemical modification of alginate: A review. Carbohydr. Polym. 2011, 84, 33-39. [CrossRef]

62. Pawar, S.N.; Edgar, K.J. Alginate derivatization: A review of chemistry, properties and applications. Biomaterials 2012, 33, 3279-3305. [CrossRef] [PubMed]

63. Vegas, A.J.; Veiseh, O.; Doloff, J.C.; Ma, M.; Tam, H.H.; Bratlie, K.; Li, J.; Bader, A.R.; Langan, E.; Olejnik, K.; et al. Combinatorial hydrogel library enables identification of materials that mitigate the foreign body response in primates. Nat. Biotechnol. 2016, 34, 345-352. [CrossRef] [PubMed]

(C) 2016 by the authors. Licensee MDPI, Basel, Switzerland. This article is an open access article distributed under the terms and conditions of the Creative Commons Attribution (CC BY) license (http:/ / creativecommons.org/licenses/by/4.0/). 
MDPI AG

St. Alban-Anlage 66

4052 Basel, Switzerland

Tel. +41616837734

Fax +41 613028918

http://www.mdpi.com

Polymers Editorial Office

E-mail: polymers@mdpi.com

http://www.mdpi.com/journal/polymers

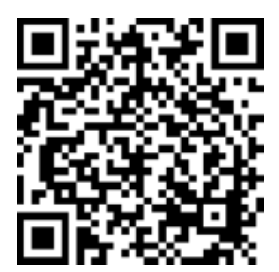



MDPI AG

St. Alban-Anlage 66

4052 Basel

Switzerland

Tel: +41 616837734

Fax: +41 613028918 